\title{
Selective Dehydrogenative Acylation of Enamides with Aldehydes Leading to Valuable $\beta$-Ketoenamides
}

\author{
Rui-Hua Liu, ${ }^{\dagger}$ Zhen-Yao Shen,${ }^{\dagger}$ Cong Wang, ${ }^{\dagger}$ Teck-Peng Loh, ${ }^{\dagger,}$ and Xu-Hong $\mathrm{Hu}^{*}, \dagger$
}

'Institute of Advanced Synthesis, School of Chemistry and Molecular Engineering, Nanjing Tech University, Nanjing 211816, China

Division of Chemistry and Biological Chemistry, School of Physical and Mathematical Sciences, Nanyang Technological University, Singapore 637371, Singapore

E-mail: ias_xhhu@njtech.edu.cn.

\section{Table of Contents}

1 General information

2 Experimental sections

2.1 General procedure for the synthesis of enamides and aldehydes 


\section{General Information}

Unless otherwise noted, all reagents and solvents were purchased from commercial suppliers and used without further purification. Specifically, iron(II) chloride (97\%), and chlorobenzene (dried over molecular sieve $4 \AA$ ) were purchased from Energy Chemical.

Thin layer chromatography was used to monitor the reaction on Merck 60 F254 precoated silica gel plate (0.2 mm thickness). TLC spots were visualized by UV-light irradiation on Spectroline Model ENF-24061/F $254 \mathrm{~nm}$. Other visualization method was staining with a basic solution of potassium permanganate, followed by heating.

Flash chromatography was performed using 200-300 mesh silica gel with the indicated solvent system.

${ }^{1} \mathrm{H}$ NMR and ${ }^{13} \mathrm{C}$ NMR spectra were recorded at $25^{\circ} \mathrm{C}$ on Bruker Advance $400 \mathrm{M} \mathrm{Hz}$ NMR and JEOL $400 \mathrm{M} \mathrm{Hz}$ spectrometers $\left(\mathrm{CDCl}_{3}\right.$ as solvent). Chemical shifts for ${ }^{1} \mathrm{H}$ NMR spectra are reported as $\delta$ in units of parts per million (ppm) downfield from $\mathrm{SiMe}_{4}(\delta 0.0)$ and relative to the signal of $\mathrm{SiMe}_{4}$ ( $\delta 0.00$ singlet). Multiplicities were given as: s (singlet), $\mathrm{d}$ (doublet), $\mathrm{t}$ (triplet), $\mathrm{q}$ (quartet), $\mathrm{dd}$ (doublet of doublets), dt (doublet of triplets), m (multiplet) and etc. Coupling constants are reported as a $J$ value in $\mathrm{Hz} .{ }^{13} \mathrm{C}$ NMR spectra are reported as $\delta$ in units of parts per million (ppm) downfield from $\mathrm{SiMe}_{4}$ $(\delta 0.0)$ and relative to the signal of chloroform- $d$ ( $\delta 77$ triplet). High resolution mass spectral analysis (HRMS) was performed on Waters-XEVOG2Q-TOF (Waters Corporation).

CAUTION: No safety issue was encountered in handling the compounds described in this work. However, extra precaution should be taken when working with peroxides which may cause explosion. 


\section{Experimental Sections}

\subsection{General procedure for the synthesis of enamides and aldehydes}<smiles>C=C(NC(C)=O)c1ccccc1</smiles>

1a<smiles>C=C(NC(C)=O)c1ccc(C#N)cc1</smiles><smiles>C=C(NC(C)=O)c1ccc2ccccc2c1</smiles>

$1 \mathrm{k}$<smiles>C=C(NC(=O)C(=O)c1ccccc1)c1ccccc1</smiles>

$1 p$<smiles>C=C(NC(C)=O)c1ccc(F)cc1</smiles>

$1 \mathrm{~b}$<smiles>C=C(NC(C)=O)c1ccc(C(F)(F)F)cc1</smiles><smiles>C=C(NC(C)=O)c1ccco1</smiles>

11<smiles>C=C(NC(C)=O)C(C)(C)C</smiles>

$1 q$<smiles>C=C(NC(C)=O)c1ccc(Cl)cc1</smiles><smiles>C=C(NC(C)=O)c1cccc(OC)c1</smiles>

$1 \mathrm{~h}$<smiles>C=C(NC(C)=O)c1cccs1</smiles>

$1 \mathrm{~m}$

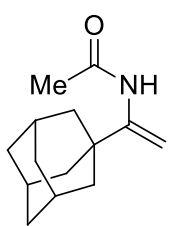

$1 \mathrm{r}$<smiles>C=C(NC(C)=O)c1ccc(Br)cc1</smiles>

$1 d$<smiles>C=C(NC(C)=O)c1ccc(C)cc1</smiles><smiles>C=C(NC(C)=O)c1ccccc1F</smiles>

1i<smiles>C=C(NC(C)=O)c1ccc2c(c1)OCO2</smiles>

$1 \mathrm{j}$<smiles>CC(=O)NC1=CCc2ccccc21</smiles><smiles>C=C(NC(=O)CC)c1ccccc1</smiles>

10

Compounds 1a-s were prepared according to the reported literatures. ${ }^{1}$

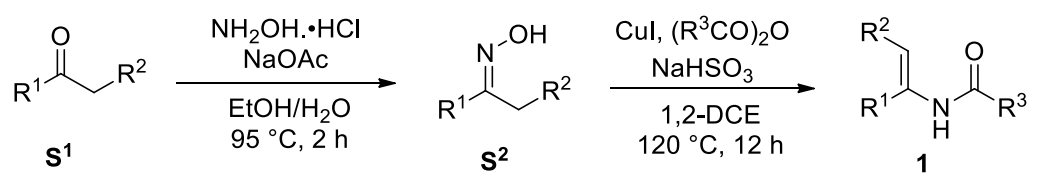

To a solution of ketone $\mathbf{S}^{\mathbf{1}}$ (10 mmol, 1.0 equiv) in EtOH- $\mathrm{H}_{2} \mathrm{O}(7.5 / 22.5 \mathrm{~mL})$ was added hydroxylamine hydrochloride (1.04 g, $15 \mathrm{mmol}, 1.5$ equiv) and NaOAc (2.05 g, $25 \mathrm{mmol}, 2.5$ equiv). After stirring at $95{ }^{\circ} \mathrm{C}$ in oil bath for $2 \mathrm{~h}$, the mixture was concentrated in vacuo and the residue was extracted with EtOAc. The organic layer was separated, washed with brine, dried over $\mathrm{Na}_{2} \mathrm{SO}_{4}$ and concentrated to give the crude product $\mathbf{S}^{2}$. A mixture of ketoxime $\mathbf{S}^{2}$ (10 mmol, 1.0 equiv), the anhydride (20 mmol, 2.0 equiv), $\mathrm{NaHSO}_{3}(3.15 \mathrm{~g}, 30 \mathrm{mmol}, 3.0$ equiv), and $\mathrm{CuI}$ (190 mg, $1 \mathrm{mmol}$, 0.1 equiv) was stirred in 1,2-DCE $(100 \mathrm{~mL})$ at $120^{\circ} \mathrm{C}$ in oil bath under $\mathrm{N}_{2}$ for $12 \mathrm{~h}$. After completion of the reaction, the reaction mixture was cooled to room temperature, diluted with EtOAc $(25 \mathrm{~mL})$, 
and washed with $2 \mathrm{M} \mathrm{NaOH}(20 \mathrm{~mL})$ and brine $(20 \mathrm{~mL})$. The organic layers were dried over $\mathrm{Na}_{2} \mathrm{SO}_{4}$ and evaporated in vacuo. The desired enamide was obtained after purification by flash chromatography on silica gel with PE/EA as the eluent.

\section{${ }^{1} \mathrm{H}$ NMR data for the enamides 1a-s:}

\section{$N$-(1-Phenylvinyl)acetamide (1a)}

Following the general procedure, 1a was obtained as a white solid. ${ }^{1} \mathrm{H}$ NMR $(400 \mathrm{MHz}$,
$3 \mathrm{H})$.

\section{$N$-(1-(4-Fluorophenyl)vinyl)acetamide (1b)}

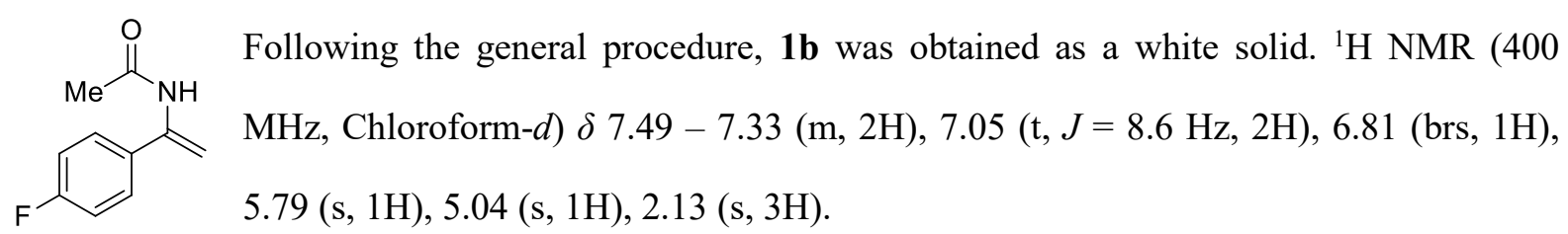

\section{$N$-(1-(4-Chlorophenyl)vinyl)acetamide (1c)}

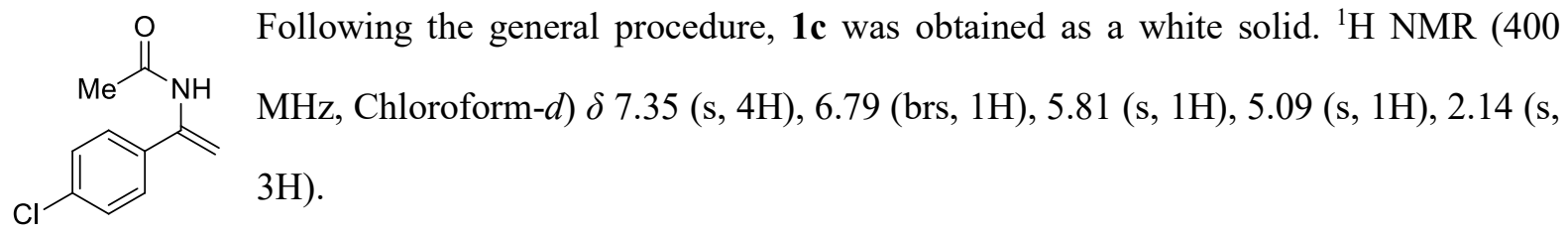

\section{$N$-(1-(4-Bromophenyl)vinyl)acetamide (1d)}

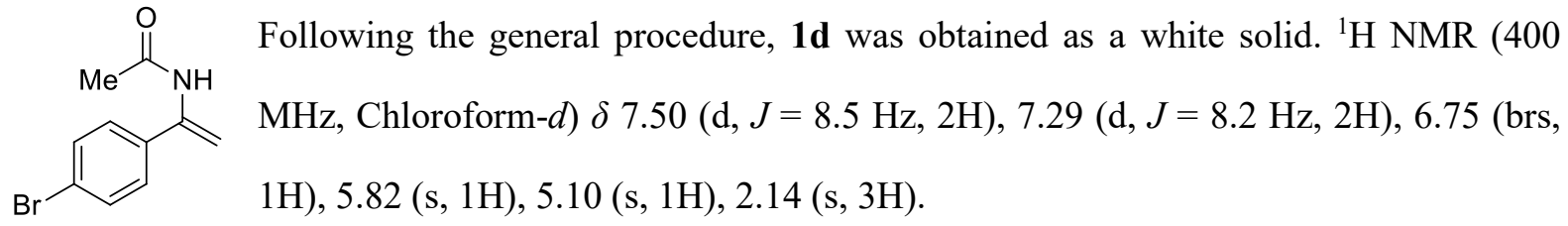

\section{$N$-(1-(p-Tolyl)vinyl)acetamide (1e)}

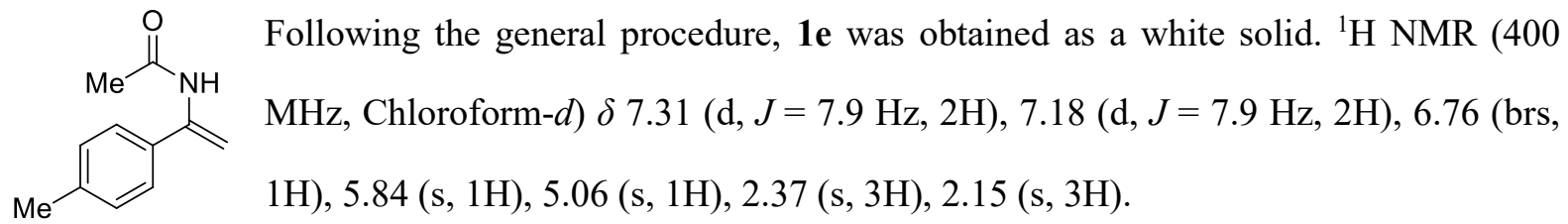




\section{$N$-(1-(4-Cyanophenyl)vinyl)acetamide (1f)}

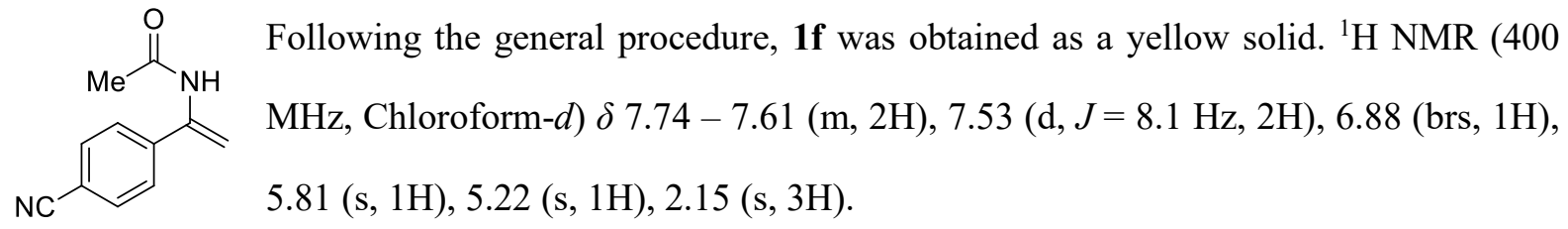

\section{$N$-(1-(4-(Trifluoromethyl)phenyl)vinyl)acetamide (1g)}

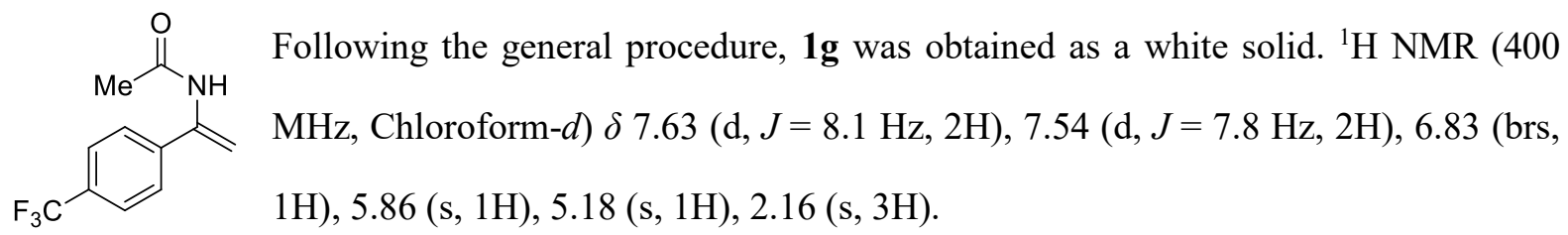

\section{$N$-(1-(3-Methoxyphenyl)vinyl)acetamide (1h)}

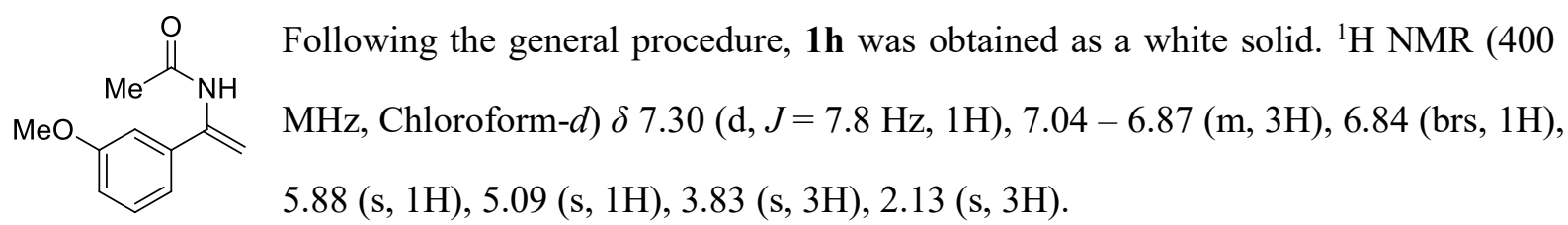

\section{$N$-(1-(2-Fluorophenyl)vinyl)acetamide (1i)}<smiles>C=C(NC(C)=O)c1ccccc1F</smiles>

Following the general procedure, $1 \mathbf{i}$ was obtained as a yellow solid. ${ }^{1} \mathrm{H}$ NMR $(400 \mathrm{MHz}$, Chloroform- $d$ ) $\delta 7.39(\mathrm{td}, J=7.7,1.7 \mathrm{~Hz}, 1 \mathrm{H}), 7.35-7.30(\mathrm{~m}, 1 \mathrm{H}), 7.15$ (tt, $J=7.6,1.2$ Hz, 1H), $7.12-7.04(\mathrm{~m}, 1 \mathrm{H}), 6.99$ (brs, 1H), $6.01(\mathrm{~s}, 1 \mathrm{H}), 5.01(\mathrm{~s}, 1 \mathrm{H}), 2.09$ (s, 3H).

\section{$N-(1-(B e n z o[d][1,3]$ dioxol-5-yl)vinyl)acetamide (1j)}<smiles>C=C(NC(C)=O)c1ccc2c(c1)OCO2</smiles>

Following the general procedure, $\mathbf{1} \mathbf{j}$ was obtained as a yellow solid. ${ }^{1} \mathrm{H}$ NMR (400 MHz, Chloroform- $d$ ) $\delta 6.90(\mathrm{~d}, J=6.8 \mathrm{~Hz}, 2 \mathrm{H}), 6.79(\mathrm{~d}, J=8.5 \mathrm{~Hz}, 2 \mathrm{H}), 5.98(\mathrm{~s}$, 2H), $5.74(\mathrm{~s}, 1 \mathrm{H}), 5.01(\mathrm{~s}, 1 \mathrm{H}), 2.13(\mathrm{~s}, 3 \mathrm{H})$.

\section{$N$-(1-(Naphthalen-2-yl)vinyl)acetamide (1k)}

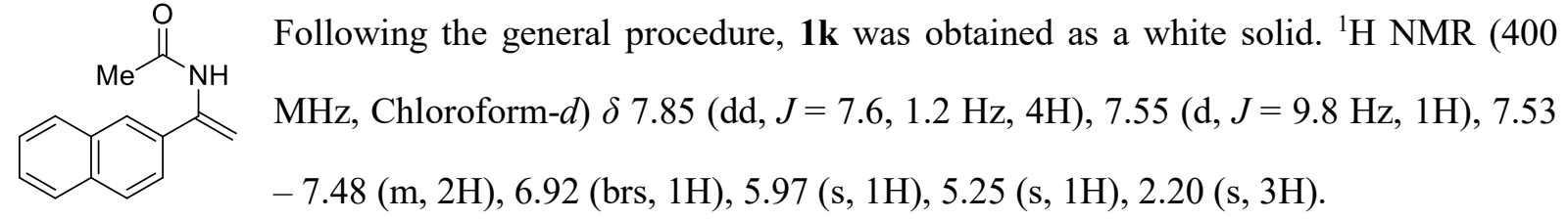




\section{$N$-(1-(Furan-2-yl)vinyl)acetamide (11)}

(1)

Following the general procedure, $\mathbf{1 l}$ was obtained as a white solid. ${ }^{1} \mathrm{H}$ NMR $(400 \mathrm{MHz}$,

Chloroform- $d$ ) $\delta 7.39(\mathrm{~s}, 1 \mathrm{H}), 7.11(\mathrm{brs}, 1 \mathrm{H}), 6.51-6.38(\mathrm{~m}, 2 \mathrm{H}), 5.91(\mathrm{~s}, 1 \mathrm{H}), 5.29(\mathrm{~s}$, $1 \mathrm{H}), 2.16(\mathrm{~s}, 3 \mathrm{H})$.

\section{$N$-(1-(Thiophen-2-yl)vinyl)acetamide (1m)}<smiles>C=C(NC(C)=O)c1cccs1</smiles>

Following the general procedure, 1m was obtained as a yellow solid. ${ }^{1} \mathrm{H}$ NMR (400 MHz, Chloroform- $d$ ) $\delta 7.24(\mathrm{~d}, J=5.2 \mathrm{~Hz}, 1 \mathrm{H}), 7.10(\mathrm{~d}, J=3.5 \mathrm{~Hz}, 1 \mathrm{H}), 7.01(\mathrm{dd}, J=5.1,3.6$ Hz, 1H), 6.81 (brs, 1H), $5.80(\mathrm{~s}, 1 \mathrm{H}), 5.24(\mathrm{~s}, 1 \mathrm{H}), 2.15(\mathrm{~s}, 3 \mathrm{H})$.

\section{$N$-(1H-inden-3-yl)acetamide (1n)}<smiles></smiles>
Following the general procedure, 1n was obtained as a brown solid. ${ }^{1} \mathrm{H}$ NMR $(400 \mathrm{MHz}$, Chloroform-d) $\delta 7.49(\mathrm{~d}, J=6.9 \mathrm{~Hz}, 2 \mathrm{H}), 7.31(\mathrm{dd}, J=14.7,7.9 \mathrm{~Hz}, 2 \mathrm{H}), 6.88(\mathrm{~s}, 1 \mathrm{H})$, $3.44(\mathrm{~d}, J=2.3 \mathrm{~Hz}, 2 \mathrm{H}), 2.25(\mathrm{~s}, 3 \mathrm{H})$.

\section{$N$-(1-Phenylvinyl)propionamide (10)}<smiles>C=C(NC(=O)CC)c1ccccc1</smiles>

Following the general procedure, 10 was obtained as a white solid. ${ }^{1} \mathrm{H}$ NMR (400 MHz, Chloroform- $d$ ) $\delta 7.46-7.32$ (m, 5H), 6.79 (brs, 1H), 5.92 (s, 1H), 5.08 (s, 1H), 2.36 (q, $J$ $=7.5 \mathrm{~Hz}, 2 \mathrm{H}), 1.23(\mathrm{t}, J=7.6 \mathrm{~Hz}, 3 \mathrm{H})$.

\section{$N$-(1-Phenylvinyl)isobutyramide (1p)}

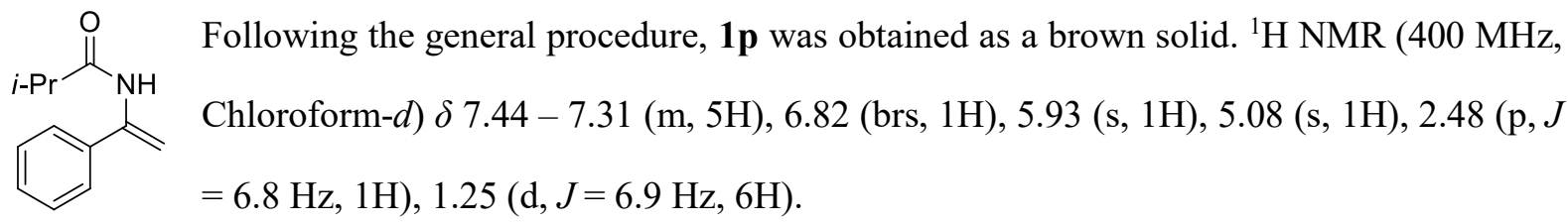

\section{$N$-(3,3-Dimethylbut-1-en-2-yl)acetamide (1q)}<smiles>CCNC(C)=O</smiles>

Following the general procedure, 1q was obtained as a white solid. ${ }^{1} \mathrm{H}$ NMR $(400 \mathrm{MHz}$, Chloroform-d) $\delta 6.44(\mathrm{brs}, 1 \mathrm{H}), 5.62(\mathrm{~s}, 1 \mathrm{H}), 4.79(\mathrm{~s}, 1 \mathrm{H}), 2.09(\mathrm{~s}, 3 \mathrm{H}), 1.12(\mathrm{~s}, 9 \mathrm{H})$.

\section{$N$-(1-((1s,3s)-Adamantan-1-yl)vinyl)acetamide (1r)}

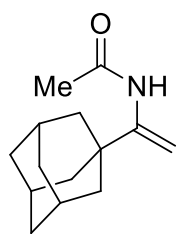

Following the general procedure, $1 \mathbf{r}$ was obtained as a white solid. ${ }^{1} \mathrm{H}$ NMR (400 MHz, Chloroform- $d$ ) $\delta 6.47(\mathrm{~s}, 1 \mathrm{H}), 5.62(\mathrm{~s}, 1 \mathrm{H}), 4.73(\mathrm{~s}, 1 \mathrm{H}), 2.08(\mathrm{~s}, 3 \mathrm{H}), 2.07$ - $2.00(\mathrm{~m}$, 
5H), $1.77-1.71(\mathrm{~m}, 6 \mathrm{H}), 1.66(\mathrm{~d}, J=12.4 \mathrm{~Hz}, 4 \mathrm{H})$.

$N$-(1-((3S,8S,9S,10R,13S,14S,17S)-3-Methoxy-10,13-dimethyl-2,3,4,7,8,9,10,11,12,13,14,15,16,17tetradecahydro-1 $H$-cyclopenta $[a]$ phenanthren-17-yl)vinyl)acetamide (1s)

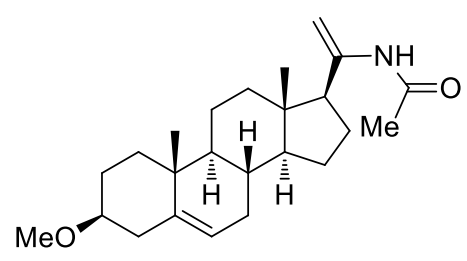

Following the general procedure, 1 s was obtained as a white solid. ${ }^{1} \mathrm{H}$ NMR (400 MHz, Chloroform- $d$ ) $\delta 6.28$ (brs, 1H), 5.82 (s, 1H), 5.36 (dd, $J=4.8,2.3 \mathrm{~Hz}, 1 \mathrm{H}), 4.65(\mathrm{~s}, 1 \mathrm{H}), 3.36(\mathrm{~s}, 3 \mathrm{H}), 3.10-3.02(\mathrm{~m}$,

$1 \mathrm{H}), 2.40$ (ddd, $J=13.2,4.7,2.3 \mathrm{~Hz}, 1 \mathrm{H}), 2.23-2.15(\mathrm{~m}, 1 \mathrm{H}), 2.13$

(s, 1H), $2.09-2.01(\mathrm{~m}, 4 \mathrm{H}), 2.01-1.82(\mathrm{~m}, 4 \mathrm{H}), 1.82-1.71(\mathrm{~m}, 2 \mathrm{H}), 1.71-1.36(\mathrm{~m}, 7 \mathrm{H}), 1.32-$ $1.17(\mathrm{~m}, 2 \mathrm{H}), 1.16-1.03(\mathrm{~m}, 1 \mathrm{H}), 1.00(\mathrm{~s}, 3 \mathrm{H}), 0.64(\mathrm{~s}, 3 \mathrm{H})$.<smiles>O=Cc1ccccc1</smiles>

$2 \mathrm{a}$<smiles>O=Cc1ccccc1Cl</smiles>

$2 \mathrm{~g}$<smiles>O=Cc1ccc(-c2ccccc2)cc1</smiles>

$2 \mathrm{~m}$<smiles>CS(C)(=O)(F)C#Cc1ccc(C=O)cc1</smiles>

$2 r$<smiles>CCCCCC=O</smiles>

$2 x$<smiles>CC1(C)OCC([C@@H]2O[C@@H]3OC(C)(C)O[C@@H]3[C@H]2OC(=O)c2ccc(C=O)cc2)O1</smiles><smiles>O=Cc1ccc(F)cc1</smiles>

$2 \mathbf{b}$<smiles>N#Cc1ccc(C=O)cc1</smiles>

2h<smiles>COc1ccc(C=O)cc1</smiles>

$2 n$<smiles>O=Cc1ccc2ccccc2c1</smiles>

2s<smiles>O=CCCc1ccccc1</smiles>

$2 y$

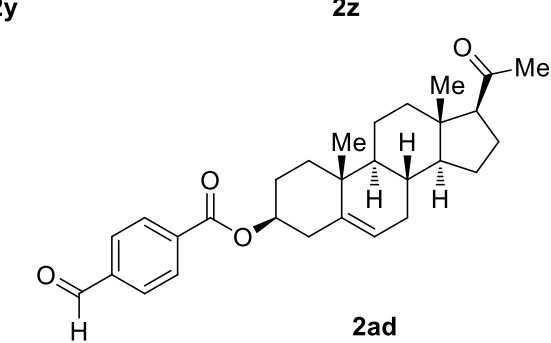<smiles>O=Cc1ccc(C(F)(F)F)cc1</smiles>

2i

2j<smiles>CCOc1ccc(C=O)cc1</smiles>

20<smiles>COc1ccc(C=O)cc1Br</smiles>

$2 p$<smiles>O=Cc1cccs1</smiles>

2t<smiles>O=Cc1ccco1</smiles>

$2 u$<smiles>O=CC1CCCCC1</smiles><smiles>O=Cc1ccc(Br)cc1</smiles><smiles>CCc1cc(C=O)ccc1F</smiles><smiles>CC(=O)c1ccc(C=O)cc1</smiles><smiles>O=Cc1ccccc1</smiles>

2k $^{\mathrm{Me}}$<smiles>Cc1ccc(C=O)cc1</smiles>

2I

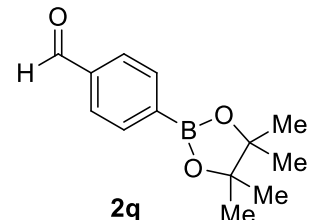<smiles>O=Cc1cn([N+](=O)[O-])c2ccccc12</smiles>

2v<smiles>CCCCCCC=O</smiles>

$2 w$<smiles>CC(C)=CCCC(C)CC=O</smiles><smiles>CC(C)[C@H]1CC[C@@H](C)C[C@H]1OC(=O)c1ccc(C=O)cc1</smiles>

2aa

$2 a b$

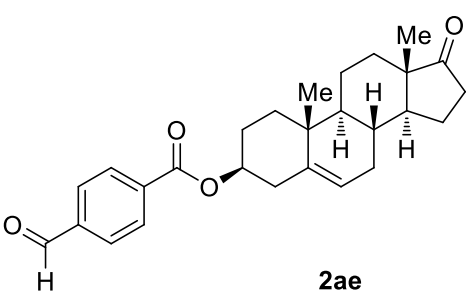


Aldehydes 2a-aa are commercial available compounds. 2ab-ae were prepared according to the reported literatures. ${ }^{2}$

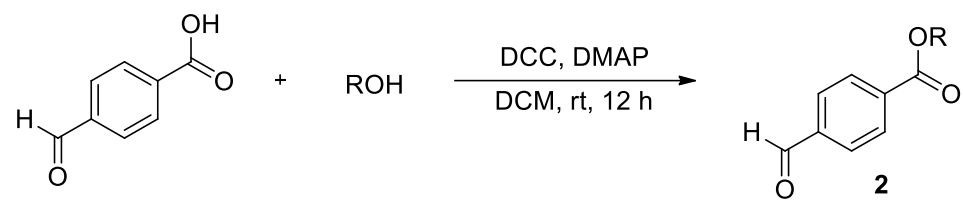

To a $50 \mathrm{~mL}$ round bottom flask was added 4-formylbenzoic acid ( $2 \mathrm{mmol}, 1.0$ equiv), alcohol (2 mmol, 1.0 equiv), DCC (2 mmol, 1.0 equiv), DMAP ( 2 mmol, 1.0 equiv) and DCM (10 mL). The reaction was stirred at room temperature for overnight. After concentration, the resultant residue was purified by flash chromatography (PE/EA) on silica gel to afford the aldehyde 2 .

\section{${ }^{1}$ H NMR data for the aldehydes 2ab-ae:}

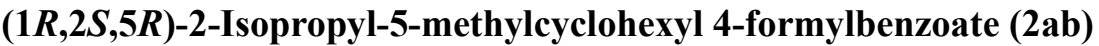<smiles>CC1CCC(C(C)C)C(OC(=O)c2ccc(C=O)cc2)C1</smiles>

Following the general procedure, 2ab was obtained as a colorless oil. ${ }^{1} \mathrm{H}$ NMR (400 MHz, Chloroform- $d$ ) $\delta 10.10$ (s, 1H), $8.22-8.17$ (m, 2H), 7.98 - 7.93 (m, 2H), 4.97 (td, $J=10.9,4.4 \mathrm{~Hz}, 1 \mathrm{H}), 2.16-2.11$ (m, 1H), $1.98-$ $1.90(\mathrm{~m}, 1 \mathrm{H}), 1.79-1.70(\mathrm{~m}, 2 \mathrm{H}), 1.61-1.51(\mathrm{~m}, 2 \mathrm{H}), 1.21-1.06(\mathrm{~m}$, $2 \mathrm{H}), 0.93(\mathrm{dd}, J=6.8,4.6 \mathrm{~Hz}, 7 \mathrm{H}), 0.80(\mathrm{~d}, J=6.9 \mathrm{~Hz}, 3 \mathrm{H})$.

(3aS,5S,6R,6aS)-5-((S)-2,2-Dimethyl-1,3-dioxolan-4-yl)-2,2-dimethyltetrahydrofuro[2,3-d] [1,3]di oxol-6-yl 4-formylbenzoate (2ac)

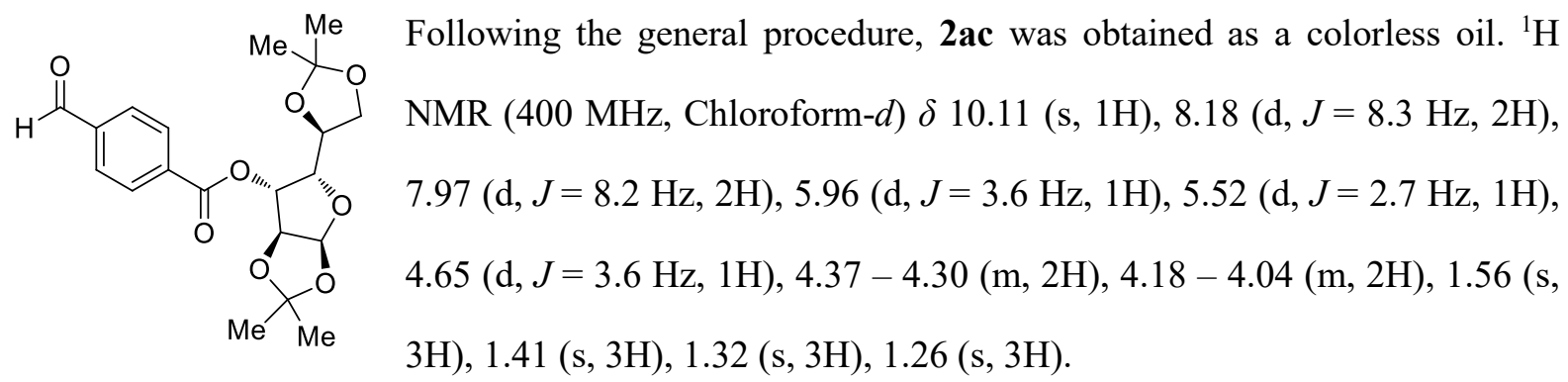




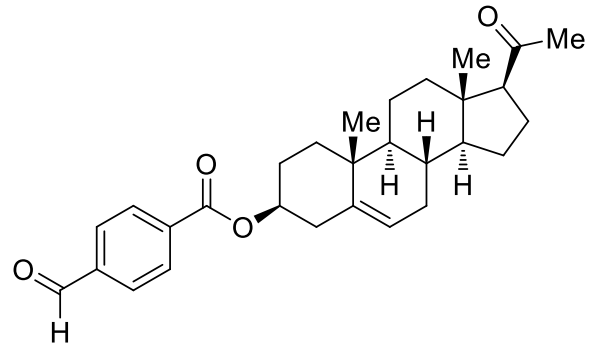

Following the general procedure, $\mathbf{2}$ ad was obtained as a white solid. ${ }^{1} \mathrm{H}$ NMR (400 MHz, Chloroform- $d$ ) $\delta 10.10$ (s, 1H), $8.20(\mathrm{~d}, J=8.3 \mathrm{~Hz}, 2 \mathrm{H}), 7.95(\mathrm{~d}, J=8.5 \mathrm{~Hz}, 2 \mathrm{H}), 5.44(\mathrm{dd}, J$ $=5.2,1.7 \mathrm{~Hz}, 1 \mathrm{H}), 4.94-4.86(\mathrm{~m}, 1 \mathrm{H}), 2.55(\mathrm{t}, J=8.9 \mathrm{~Hz}$, 1H), $2.49(\mathrm{~d}, J=7.7 \mathrm{~Hz}, 2 \mathrm{H}), 2.24-2.15(\mathrm{~m}, 1 \mathrm{H}), 2.14(\mathrm{~s}$, 3H), $2.07-2.00(\mathrm{~m}, 2 \mathrm{H}), 1.95(\mathrm{dt}, J=13.4,3.7 \mathrm{~Hz}, 1 \mathrm{H}), 1.83-1.61(\mathrm{~m}, 5 \mathrm{H}), 1.59-1.41(\mathrm{~m}, 4 \mathrm{H})$, $1.31-1.13(\mathrm{~m}, 3 \mathrm{H}), 1.08(\mathrm{~s}, 3 \mathrm{H}), 1.07-1.00(\mathrm{~m}, 1 \mathrm{H}), 0.65(\mathrm{~s}, 3 \mathrm{H})$.

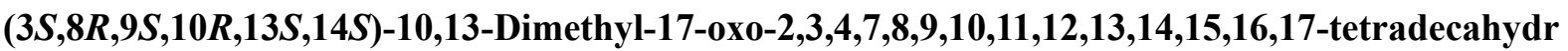
o-1H-cyclopenta[a]phenanthren-3-yl 4-formylbenzoate (2ae)

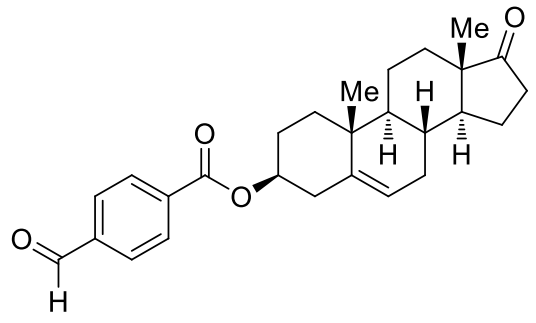

Following the general procedure, 2ae was obtained as a white solid. ${ }^{1} \mathrm{H}$ NMR (400 MHz, Chloroform- $d$ ) $\delta 10.11$ (s, 1H), 8.20 $(\mathrm{d}, J=8.4 \mathrm{~Hz}, 2 \mathrm{H}), 7.95(\mathrm{~d}, J=8.4 \mathrm{~Hz}, 2 \mathrm{H}), 5.47(\mathrm{~d}, J=5.2 \mathrm{~Hz}$ 1H), $4.96-4.84(\mathrm{~m}, 1 \mathrm{H}), 2.51(\mathrm{dd}, J=7.2,2.4 \mathrm{~Hz}, 2 \mathrm{H}), 2.49-$ $2.42(\mathrm{~m}, 1 \mathrm{H}), 2.20-1.84(\mathrm{~m}, 6 \mathrm{H}), 1.84-1.75(\mathrm{~m}, 1 \mathrm{H}), 1.74-$ $1.68(\mathrm{~m}, 3 \mathrm{H}), 1.61-1.49(\mathrm{~m}, 3 \mathrm{H}), 1.37-1.23(\mathrm{~m}, 3 \mathrm{H}), 1.11(\mathrm{~s}, 3 \mathrm{H}), 0.91(\mathrm{~s}, 3 \mathrm{H})$.

Table S1. Effect of Base on the Reaction ${ }^{a}$

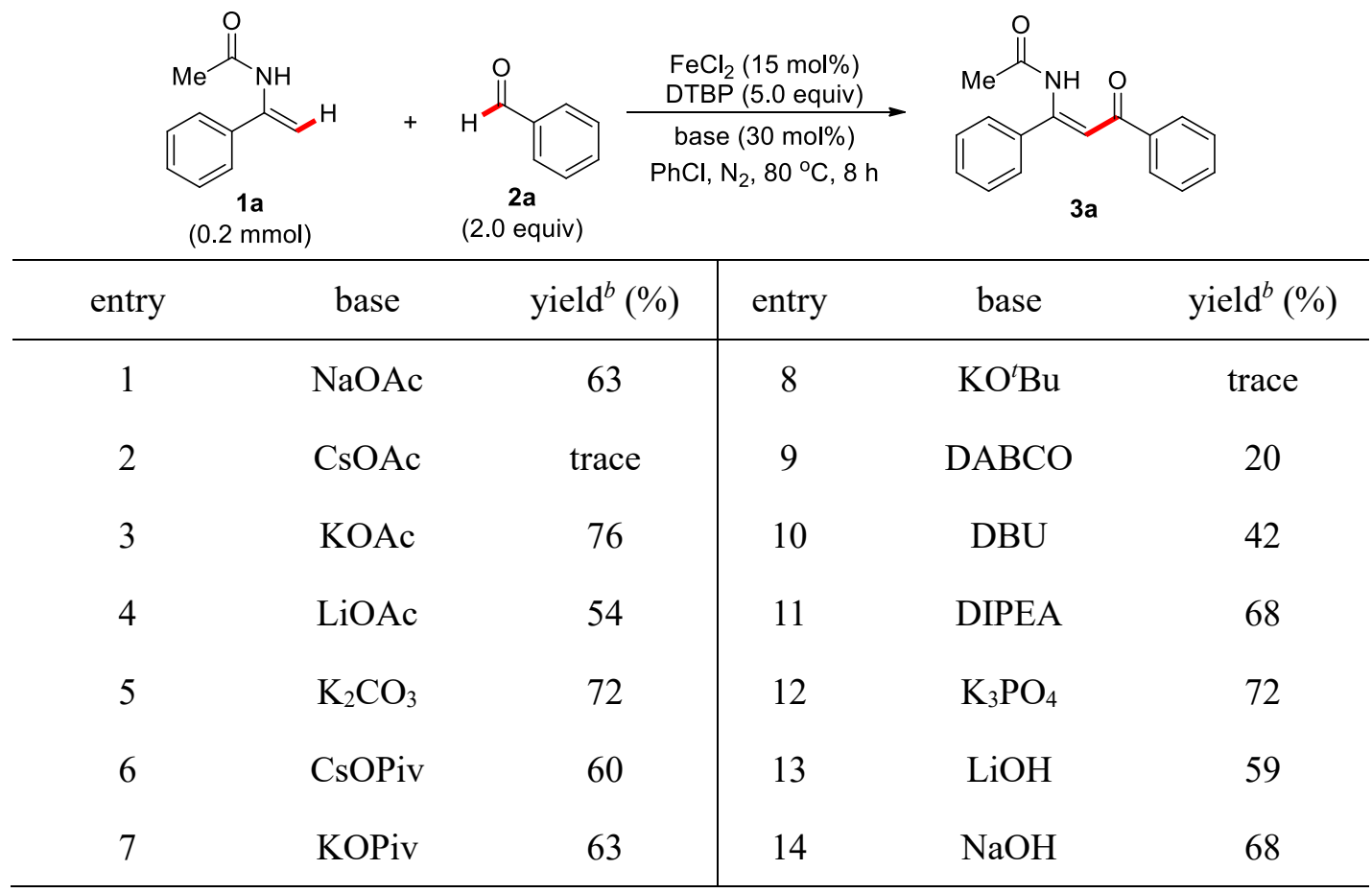


${ }^{a}$ Unless otherwise noted, all reactions were conducted with $\mathbf{1 a}(0.2 \mathrm{mmol}), \mathbf{2 a}\left(2.0\right.$ equiv), $\mathrm{FeCl}_{2}\left(15 \mathrm{~mol}^{\circ}\right)$, DTBP (5.0 equiv), and base $(30 \mathrm{~mol} \%)$ in $\mathrm{PhCl}(0.8 \mathrm{~mL})$ under $\mathrm{N}_{2}$ at $80{ }^{\circ} \mathrm{C}$ for $8 \mathrm{~h} .{ }^{b}$ Isolated yield.

Table S2. Effect of Solvent on the Reaction ${ }^{a}$

\begin{tabular}{|c|c|c|c|c|c|}
\hline entry & solvent & $\operatorname{yield}^{b}(\%)$ & entry & solvent & $\operatorname{yield}^{b}(\%)$ \\
\hline 1 & DMSO & 23 & 5 & toluene & 46 \\
\hline 2 & $\mathrm{MeCN}$ & 33 & 6 & $\mathrm{PhCF}_{3}$ & 32 \\
\hline 3 & 1,2-DCE & 51 & 7 & $o-\mathrm{Cl}_{2}-\mathrm{C}_{6} \mathrm{H}_{4}$ & 67 \\
\hline 4 & THF & trace & 8 & $\mathrm{PhCl}$ & 76 \\
\hline
\end{tabular}

${ }^{a}$ Unless otherwise noted, all reactions were conducted with $\mathbf{1 a}(0.2 \mathrm{mmol}), \mathbf{2 a}\left(2.0\right.$ equiv), $\mathrm{FeCl}_{2}\left(15 \mathrm{~mol}^{\circ}\right)$, DTBP (5.0 equiv), and KOAc (30 mol\%) in solvent $(0.8 \mathrm{~mL})$ under $\mathrm{N}_{2}$ at $80{ }^{\circ} \mathrm{C}$ for $8 \mathrm{~h} .{ }^{b}$ Isolated yield.

\subsection{Typical procedure for the synthesis of 3a-ae and 4a-r}
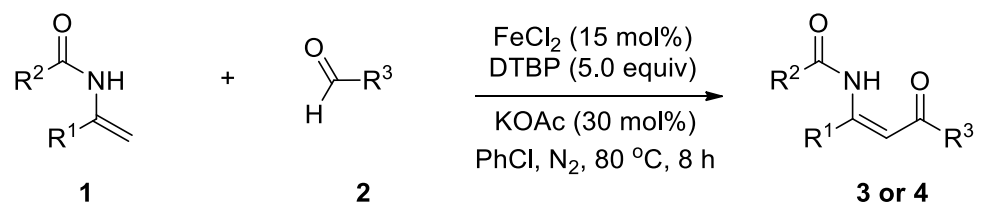

A $10 \mathrm{~mL}$ screw-capped Schlenk tube was charged with enamide 1 ( $0.2 \mathrm{mmol}, 1.0$ equiv), $\mathrm{FeCl}_{2}$ (4.0 mg, $0.03 \mathrm{mmol}, 15 \mathrm{~mol} \%$ ), aldehyde 2 (0.4 mmol, 2.0 equiv), DTBP (146.3 mg, $1 \mathrm{mmol}, 5.0$ equiv), $\mathrm{KOAc}\left(5.9 \mathrm{mg}, 0.06 \mathrm{mmol}, 0.3\right.$ equiv) in $\mathrm{PhCl}(0.8 \mathrm{~mL})$. The mixture was stirred at $80{ }^{\circ} \mathrm{C}$ in oil bath for $8 \mathrm{~h}$ under $\mathrm{N}_{2}$ atmosphere. After full conversion, the mixture was cooled down and concentrated under vacuum directly. The resultant residue was purified by flash chromatography (PE/EA) on silica gel to afford the product 3 or 4. 


\section{Derivatization of the product}

\subsection{Synthesis of oxazole $5 a^{3}$}

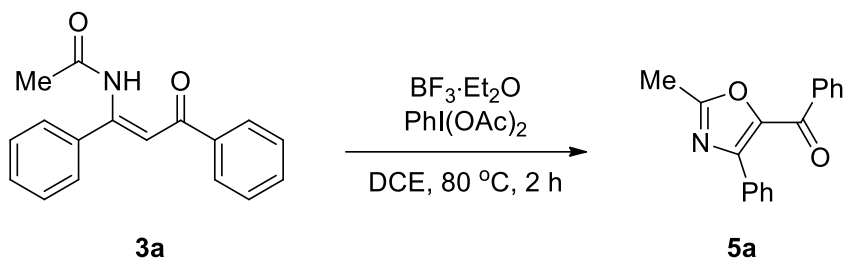

To a solution of compound 3a (39.8 mg, $0.15 \mathrm{mmol}, 1.0$ equiv) in anhydrous 1,2-DCE (1.5 mL) was added $\mathrm{BF}_{3} \cdot \mathrm{Et}_{2} \mathrm{O}$ (116.1 mg, $0.375 \mathrm{mmol}, 2.5$ equiv) and $\mathrm{PhI}(\mathrm{OAc})_{2}(72.5 \mathrm{mg}, 0.225 \mathrm{mmol}, 1.5$ equiv). The reaction mixture was stirred at $80{ }^{\circ} \mathrm{C}$ in oil bath for $2 \mathrm{~h}$. The reaction mixture was cooled down to room temperature, quenched with sat. aq. $\mathrm{NaHCO}_{3}$, and then extracted with DCM. The combined organic layer was washed with sat. $\mathrm{NaCl}$, dried over $\mathrm{Na}_{2} \mathrm{SO}_{4}$ and concentrated under vacuum. The resultant residue was purified by flash column chromatography (PE/EA) to give the product 5a as a white solid (36.9 $\mathrm{mg}, 94 \%$ yield). ${ }^{1} \mathrm{H}$ NMR (400 MHz, Chloroform- $d$ ) $\delta 7.94-7.88$ (m, 2H), $7.87-7.83(\mathrm{~m}, 2 \mathrm{H}), 7.57-7.50(\mathrm{~m}, 1 \mathrm{H}), 7.40(\mathrm{t}, J=7.8 \mathrm{~Hz}, 2 \mathrm{H}), 7.37-7.32(\mathrm{~m}, 3 \mathrm{H}), 2.61$ (s, 3H). ${ }^{13} \mathrm{C}$ NMR (101 MHz, Chloroform- $d$ ) $\delta 183.2,162.9,147.6,143.7,137.2,132.8,130.5,129.6$, $129.4 \times 2,129.1 \times 2,128.2 \times 2,128.1 \times 2,14.3 . \mathrm{HRMS}(\mathrm{ESI}): \mathrm{m} / \mathrm{z}$ calculated for $\mathrm{C}_{17} \mathrm{H}_{14} \mathrm{NO}_{2}[\mathrm{M}+$ $\mathrm{H}]^{+}:$264.1025, found: 264.1029 .

\subsection{Synthesis of pyridine $5 b^{4}$}

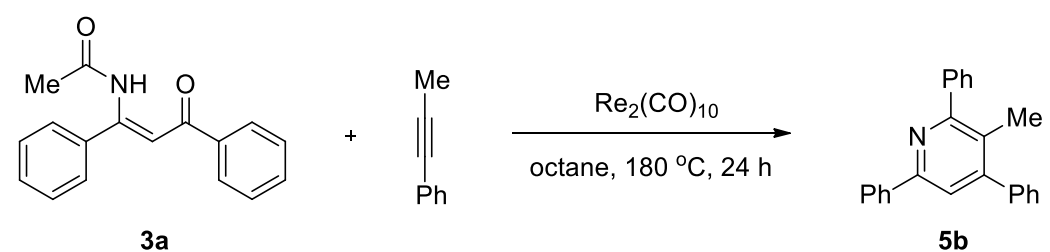

A mixture of $\operatorname{Re}_{2}(\mathrm{CO})_{10}(13.1 \mathrm{mg}, 0.02 \mathrm{mmol}, 0.1$ equiv), 3a (90.2 mg, $0.34 \mathrm{mmol}, 1.7$ equiv), 1-phenylpropyne (23.2 mg, $0.20 \mathrm{mmol}, 1.0$ equiv), and octane $(0.5 \mathrm{~mL})$ was stirred at $180{ }^{\circ} \mathrm{C}$ in oil bath for $24 \mathrm{~h}$ under $\mathrm{N}_{2}$ atmosphere. After the reaction mixture was cooled to room temperature, the solvent was removed in vacuo. The resultant residue was purified by flash column chromatography (PE/EA) to give the product $\mathbf{5 b}$ as a yellow solid (51.9 $\mathrm{mg}, 81 \%$ yield). ${ }^{1} \mathrm{H}$ NMR (400 MHz, Chloroform- $d$ ) $\delta 8.11(\mathrm{dd}, J=8.3,1.4 \mathrm{~Hz}, 2 \mathrm{H}), 7.71(\mathrm{dd}, J=8.2,1.5 \mathrm{~Hz}, 2 \mathrm{H}), 7.64(\mathrm{~s}, 1 \mathrm{H}), 7.55-$ $7.40(\mathrm{~m}, 11 \mathrm{H}), 2.29(\mathrm{~s}, 3 \mathrm{H}) .{ }^{13} \mathrm{C}$ NMR (101 MHz, Chloroform-d) $\delta 159.4,154.0,151.6,141.4,140.25$, $139.3,130.3,130.1,129.4 \times 2,128.8 \times 2,128.6 \times 2,128.4 \times 2,128.0 \times 2,127.9 \times 2,127.4,126.9$, 


\subsection{Synthesis of 2-pyridone $5 c^{5}$}<smiles>CC(=O)N/C(=C\C(=O)c1ccccc1)c1ccccc1</smiles>

$3 \mathbf{a}$

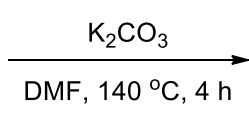

To a solution of compound 3a (39.8 mg, $0.15 \mathrm{mmol}, 1.0$ equiv) in anhydrous DMF ( $0.5 \mathrm{~mL})$ was added potassium carbonate $\left(20.8 \mathrm{mg}, 0.15 \mathrm{mmol}, 1.0\right.$ equiv). The mixture was stirred at $140{ }^{\circ} \mathrm{C}$ in oil bath for $4 \mathrm{~h}$. After cooling down, the reaction was quenched with water and $\mathrm{pH}$ value was adjusted to 3-4 by $2 \mathrm{~N} \mathrm{HCl}$ solution. Then the mixture was extracted with EtOAc $(10 \mathrm{~mL} \times 3)$ and rinsed with sat. aq. $\mathrm{NaCl}(10 \mathrm{~mL})$. The combined organic layers were dried over $\mathrm{Na}_{2} \mathrm{SO}_{4}$ and concentrated under vacuum. The crude product was purified by flash column chromatography (PE/EA) to afford $\mathbf{5 c}$ as a colorless oil (22.4 mg, 61\% yield). ${ }^{1} \mathrm{H}$ NMR (400 MHz, Chloroform- $d$ ) $\delta 12.27$ (s, 1H), 7.87 - 7.75 $(\mathrm{m}, 2 \mathrm{H}), 7.71-7.61(\mathrm{~m}, 2 \mathrm{H}), 7.59-7.41(\mathrm{~m}, 6 \mathrm{H}), 6.83-6.71(\mathrm{~m}, 2 \mathrm{H}) .{ }^{13} \mathrm{C}$ NMR $(101 \mathrm{MHz}$, Chloroform- $d$ ) $\delta 165.6,153.7,146.9,138.1,133.7,130.1,129.4,129.1 \times 2,129.0 \times 2,126.9 \times 2$, $126.8 \times 2$, 115.2, 104.9. HRMS $(\mathrm{ESI}): \mathrm{m} / \mathrm{z}$ calculated for $\mathrm{C}_{17} \mathrm{H}_{14} \mathrm{NO}[\mathrm{M}+\mathrm{H}]^{+}: 248.1075$, found: 248.1079 .

\subsection{Synthesis of enaminone $5 \mathrm{~d}^{6}$}<smiles>CC(=O)N/C(=C\C(=O)c1ccccc1)c1ccccc1</smiles>

$3 \mathbf{a}$

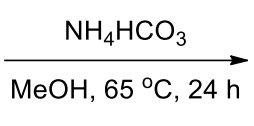

To a solution of compound 3a (26.5 mg, $0.1 \mathrm{mmol}, 1.0$ equiv) in $\mathrm{MeOH}(1.0 \mathrm{~mL})$ was added $\mathrm{NH}_{4} \mathrm{HCO}_{3}\left(0.126 \mathrm{~g}, 1.6 \mathrm{mmol}, 16.0\right.$ equiv). The reaction mixture was stirred at $65{ }^{\circ} \mathrm{C}$ in oil bath for $24 \mathrm{~h}$. Upon completion, the mixture was then concentrated under vacuum and the residue was purified by chromatography on silica gel (PE/EA) to afford $\mathbf{5 d}$ as a yellow oil $\left(18.7 \mathrm{mg}, 84 \%\right.$ yield). ${ }^{1} \mathrm{H}$ NMR (400 MHz, Chloroform- $d$ ) $\delta 10.43$ (brs, 1H), 7.96 (dd, $J=7.9,1.7 \mathrm{~Hz}, 2 \mathrm{H}), 7.65$ (dd, $J=7.9,1.6 \mathrm{~Hz}$, 2H), $7.54-7.41(\mathrm{~m}, 6 \mathrm{H}), 6.16$ (s, 1H), 5.52 (brs, 1H). ${ }^{13} \mathrm{C}$ NMR (101 MHz, Chloroform-d) $\delta 190.2$, $162.9,140.3,137.6,131.0,130.7,129.0 \times 2,128.3 \times 2,127.2 \times 2,126.3 \times 2,91.9$. HRMS $($ ESI): $\mathrm{m} / \mathrm{z}$ 
calculated for $\mathrm{C}_{15} \mathrm{H}_{14} \mathrm{NO}[\mathrm{M}+\mathrm{H}]^{+}: 224.1075$, found: 224.1074 .

\subsection{Synthesis of pyrazole $5 \mathrm{e}^{7}$}

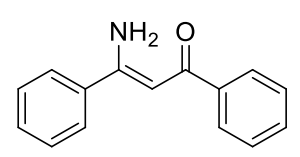

5d

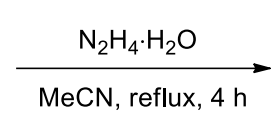

CN, reflux, $4 \mathrm{~h}$

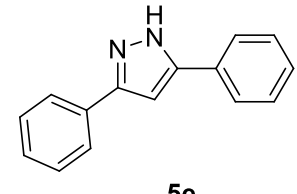

$5 e$

To a solution of enaminone $\mathbf{5 d}(30.2 \mathrm{mg}, 0.13 \mathrm{mmol})$ in $\mathrm{MeCN}(0.8 \mathrm{~mL})$ was added hydrazine hydrate $(0.2 \mathrm{~mL})$. The mixture was refluxed for $4 \mathrm{~h}$. The reaction mixture was cooled to room temperature and concentrated under vacuum. The crude product was purified by flash column chromatography (PE/EA) to afford 5e as a white solid (26.4 mg, 92\% yield). ${ }^{1} \mathrm{H}$ NMR (401 MHz, Chloroform- $d) \delta 7.74(\mathrm{~d}, J=6.9 \mathrm{~Hz}, 4 \mathrm{H}), 7.46-7.39(\mathrm{~m}, 4 \mathrm{H}), 7.38-7.32(\mathrm{~m}, 2 \mathrm{H}), 6.85(\mathrm{~s}, 1 \mathrm{H}) .{ }^{13} \mathrm{C}$ NMR $(101 \mathrm{MHz}$, Chloroform- $d) \delta 128.8 \times 6,128.1 \times 2,125.6 \times 6,100.0$. HRMS $(\mathrm{ESI}): \mathrm{m} / \mathrm{z}$ calculated for $\mathrm{C}_{15} \mathrm{H}_{12} \mathrm{~N}_{2}[\mathrm{M}+\mathrm{H}]^{+}: 221.1079$, found: 221.1083 .

\subsection{Synthesis of 1,3-amino alcohol $5 f^{8}$}

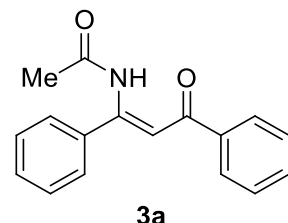

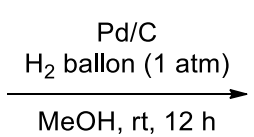

$\mathrm{MeOH}, \mathrm{rt}, 12 \mathrm{~h}$

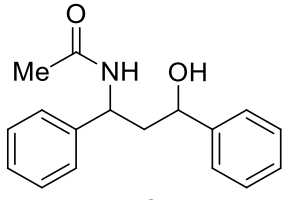

$5 f$

A solution of compound 3a $(26.5 \mathrm{mg}, 0.1 \mathrm{mmol})$ in $\mathrm{MeOH}(1.0 \mathrm{~mL})$ was treated with $\mathrm{Pd} / \mathrm{C}(4.0$ $\mathrm{mg}, 10 \mathrm{wt} \%$ ) and then placed under a hydrogen balloon and vigorously stirred at room temperature for $12 \mathrm{~h}$. The heterogeneous solution was filtered through a small pad of Celite ${ }^{\circledR}$. Removals of the solvent under vacuum and purification of the residue by flash column chromatography (PE/EA) afforded $\mathbf{5 f}$ as a white solid (26.1 mg, 96\% yield). ${ }^{1} \mathrm{H}$ NMR (401 MHz, Chloroform- $d$ ) $\delta 7.38-7.29$ (m, 9H), $7.28-7.23(\mathrm{~m}, 1 \mathrm{H}), 6.30(\mathrm{~d}, J=8.4 \mathrm{~Hz}, 1 \mathrm{H}), 5.33(\mathrm{td}, J=9.2,3.7 \mathrm{~Hz}, 1 \mathrm{H}), 4.68(\mathrm{dt}, J=$ 10.2, 3.2 Hz, 1H), 4.13 (d, $J=3.5 \mathrm{~Hz}, 1 \mathrm{H}), 2.20-2.08(\mathrm{~m}, 2 \mathrm{H}), 2.08(\mathrm{~s}, 3 \mathrm{H}) 1.67$ (brs, $1 \mathrm{H}) .{ }^{13} \mathrm{C}$ NMR $(101 \mathrm{MHz}$, Chloroform- $d$ ) $\delta 170.8,143.8,141.0,128.9 \times 2,128.4 \times 2,127.7,127.4,126.6 \times 2,125.7$ $\times 2,70.4,51.2,45.8,23.4$. HRMS (ESI): $\mathrm{m} / \mathrm{z}$ calculated for $\mathrm{C}_{17} \mathrm{H}_{20} \mathrm{NO}_{2}[\mathrm{M}+\mathrm{H}]^{+}: 270.1494$, found: 270.1489 . 


\subsection{Mechanistic Studies}

\subsubsection{Large scale synthesis of 3a}

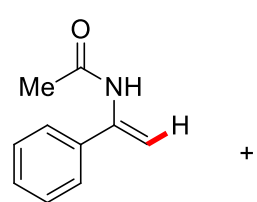

$1 \mathrm{a}$<smiles>O=Cc1ccccc1</smiles>

$2 a$

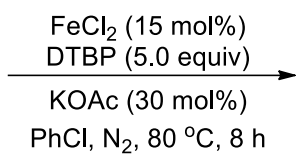

$\mathrm{PhCl}, \mathrm{N}_{2}, 80^{\circ} \mathrm{C}, 8 \mathrm{~h}$

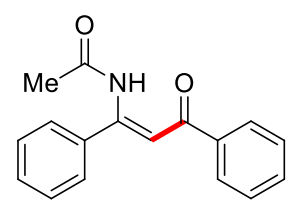

$3 a$

A $10 \mathrm{~mL}$ screw-capped Schlenk tube was charged with enamide 1a $(161.2 \mathrm{mg}, 1.0 \mathrm{mmol}, 1.0$ equiv), $\mathrm{FeCl}_{2}$ (19.0 mg, $0.15 \mathrm{mmol}, 15 \mathrm{~mol} \%$ ), aldehyde $\mathbf{2 a}$ (212.2 mg, $2.0 \mathrm{mmol}, 2.0$ equiv), DTBP (731.2 mg, $5.0 \mathrm{mmol}, 5.0$ equiv), KOAc (29.4 mg, $0.3 \mathrm{mmol}, 0.3$ equiv) in $\mathrm{PhCl}(4.0 \mathrm{~mL})$. The mixture was stirred at $80{ }^{\circ} \mathrm{C}$ in oil bath for $8 \mathrm{~h}$ under $\mathrm{N}_{2}$ atmosphere. After full conversion, the mixture was cooled down and concentrated under vacuum directly. The resultant residue was purified by flash chromatography (PE/EA) on silica gel to afford the product 3a (196.1 mg, $0.74 \mathrm{mmol}, 74 \%$ ).

\subsubsection{Acylation of tertiary enamide $1 \mathrm{~s}$}

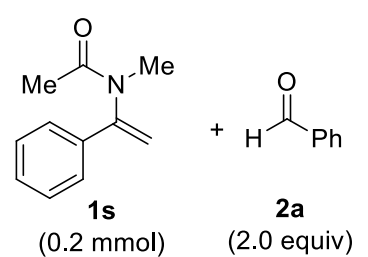

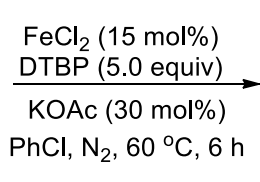

$\mathrm{PhCl}, \mathrm{N}_{2}, 60^{\circ} \mathrm{C}, 6 \mathrm{~h}$

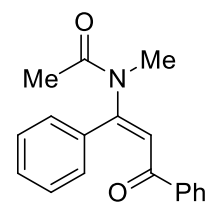

4s, $41 \%$

A $10 \mathrm{~mL}$ screw-capped Schlenk tube was charged with the enamide $1 \mathrm{~s}$ (35.0 $\mathrm{mg}, 0.2 \mathrm{mmol}, 1.0$ equiv), $\mathrm{FeCl}_{2}$ (4.0 mg, $0.03 \mathrm{mmol}, 15 \mathrm{~mol} \%$ ), benzaldehyde $2 \mathrm{a}$ (42.4 mg, $0.4 \mathrm{mmol}, 2.0$ equiv), DTBP (146.3 mg, $1 \mathrm{mmol}, 5.0$ equiv), KOAc ( $5.9 \mathrm{mg}, 0.06 \mathrm{mmol}, 0.3$ equiv) in $\mathrm{PhCl}(0.8 \mathrm{~mL})$. The mixture was stirred at $60{ }^{\circ} \mathrm{C}$ in oil bath for $6 \mathrm{~h}$ under $\mathrm{N}_{2}$ atmosphere. After full conversion, the mixture was cooled down and concentrated under vacuum directly. The resultant residue was purified by flash chromatography (PE/EA) on silica gel to afford the product $4 \mathbf{s}$ as a yellow oil $(22.7 \mathrm{mg}, 0.082 \mathrm{mmol}$, 41\%). ${ }^{1} \mathrm{H}$ NMR (401 MHz, Chloroform- $d$ ) $\delta 7.87(\mathrm{dd}, J=8.4,1.3 \mathrm{~Hz}, 2 \mathrm{H}), 7.53-7.48(\mathrm{~m}, 1 \mathrm{H}), 7.42$ - $7.36(\mathrm{~m}, 2 \mathrm{H}), 7.35-7.28(\mathrm{~m}, 2 \mathrm{H}), 7.28-7.25(\mathrm{~m}, 3 \mathrm{H}), 6.63(\mathrm{~s}, 1 \mathrm{H}), 3.10(\mathrm{~s}, 3 \mathrm{H}), 2.19(\mathrm{~s}, 3 \mathrm{H}) .{ }^{13} \mathrm{C}$ NMR (101 MHz, Chloroform-d) $\delta$ 191.9, 171.1, 153.2, 137.4, 134.4, 133.2, 130.3, $128.9 \times 2,128.7 \times$ $2,128.7 \times 2,128.6 \times 2,122.5,36.0,22.9$. HRMS $(E S I): \mathrm{m} / \mathrm{z}$ calculated for $\mathrm{C}_{18} \mathrm{H}_{18} \mathrm{NO}_{2}[\mathrm{M}+\mathrm{H}]^{+}:$ 280.1338 , found: 280.1334 . 


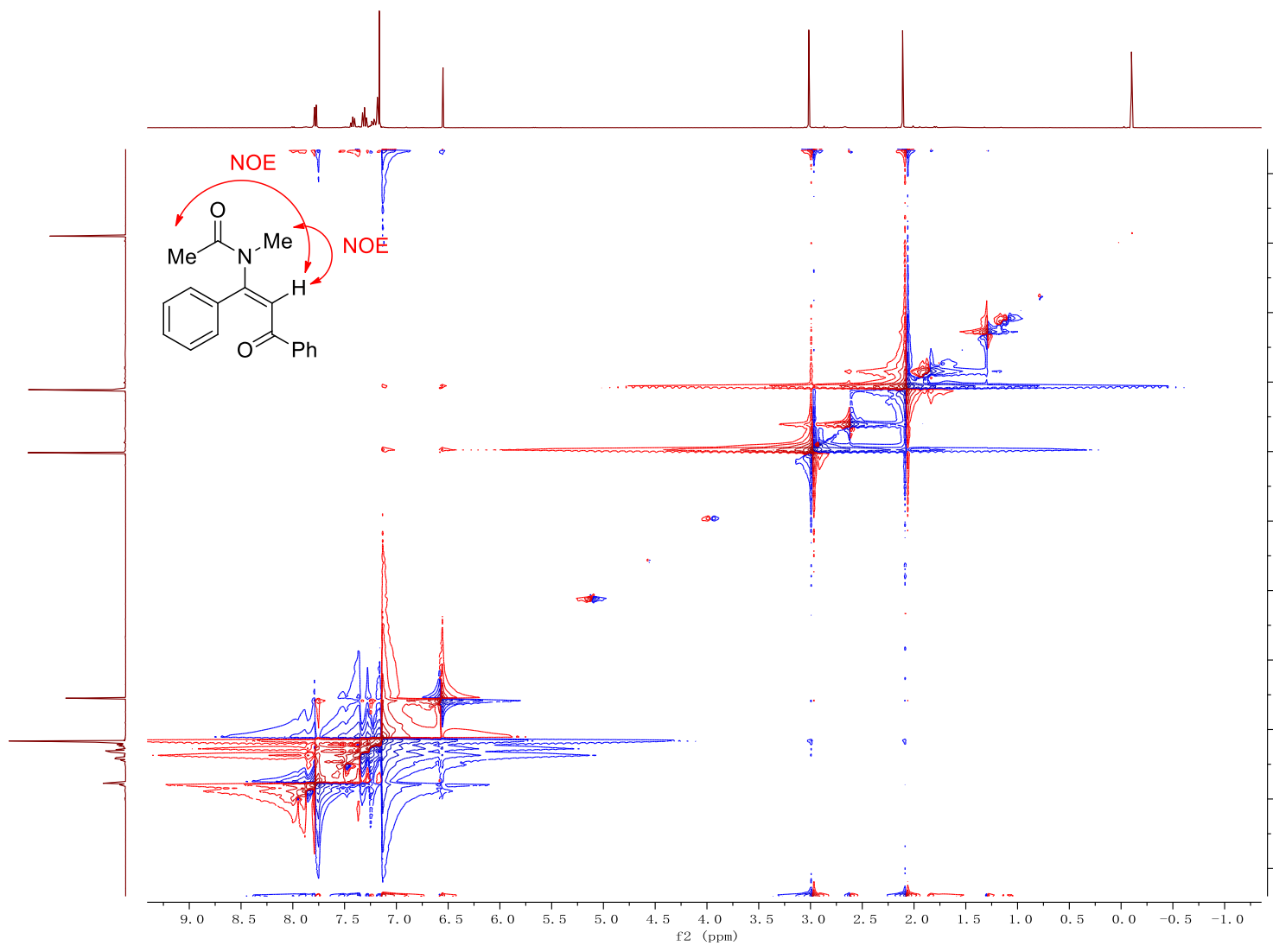

NOESY analysis of $4 \mathrm{~s}$

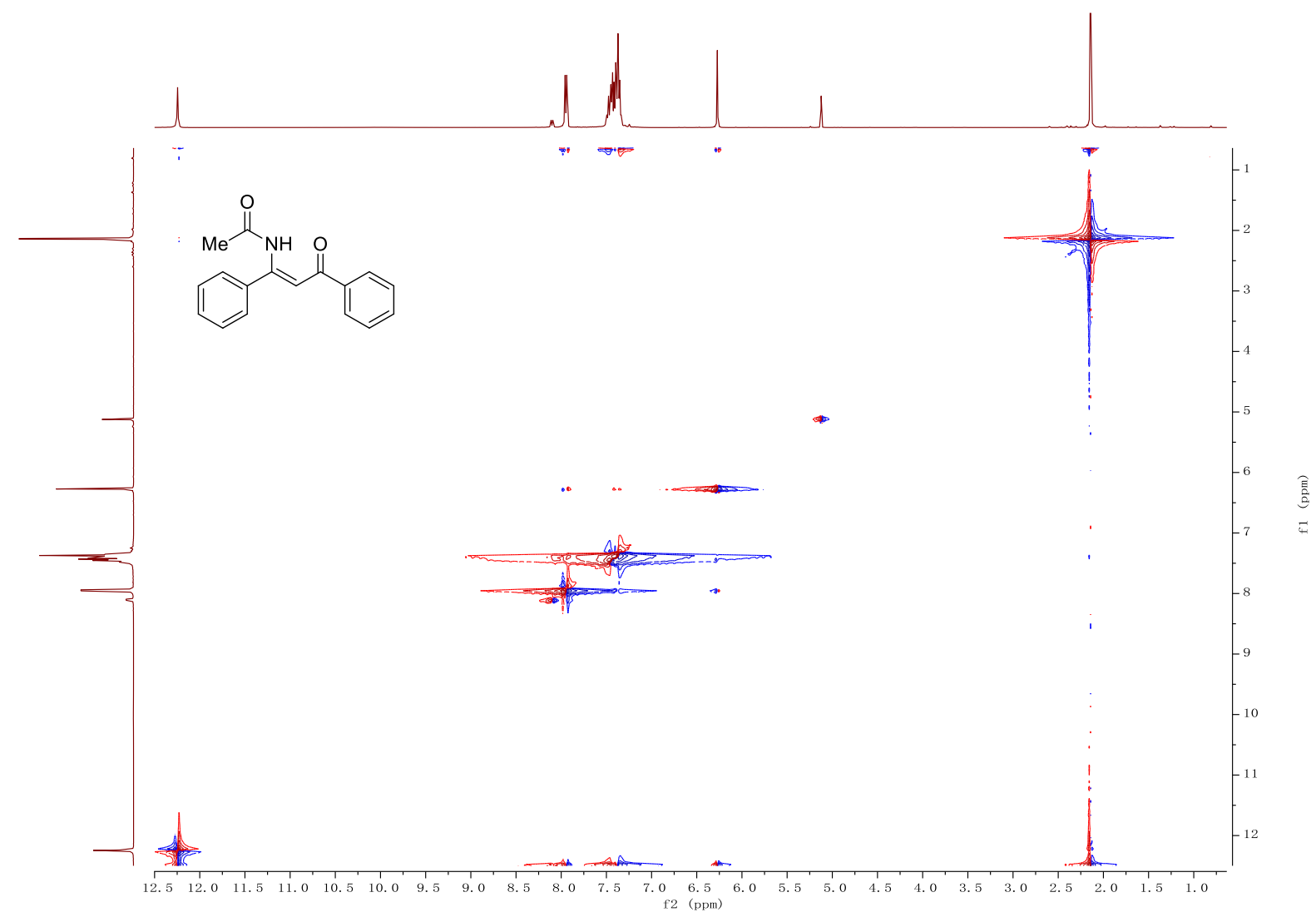

NOESY analysis of 3a 


\subsubsection{Radical-trapping experiments}

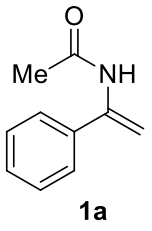

$(0.2 \mathrm{mmol})$

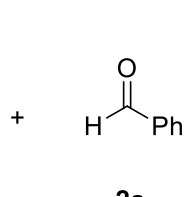

(2.0 equiv)
$\mathrm{FeCl}_{2}(15 \mathrm{~mol} \%)$

DTBP (5.0 equiv)

$\underset{\operatorname{KOAC}(30 \mathrm{~mol} \%)}{\stackrel{\text { TEMPO }(2.0 \text { equiv })}{\longrightarrow}}$

$\mathrm{PhCl}, \mathrm{N}_{2}, 80^{\circ} \mathrm{C}, 8 \mathrm{~h}$

A $10 \mathrm{~mL}$ screw-capped Schlenk tube was charged with the enamide 1a $(32.3 \mathrm{mg}, 0.2 \mathrm{mmol}, 1.0$ equiv), $\mathrm{FeCl}_{2}$ (4.0 mg, $0.03 \mathrm{mmol}, 15 \mathrm{~mol} \%$ ), benzaldehyde $2 \mathrm{a}$ (42.5 mg, $0.4 \mathrm{mmol}, 2.0$ equiv), DTBP (146.3 mg, $1 \mathrm{mmol}, 5.0$ equiv), KOAc (5.9 mg, $0.06 \mathrm{mmol}, 0.3$ equiv), TEMPO (62.5 mg, $0.4 \mathrm{mmol}$, 2.0 equiv) in $\mathrm{PhCl}(0.8 \mathrm{~mL})$. The mixture was stirred at $80^{\circ} \mathrm{C}$ in oil bath for $8 \mathrm{~h}$ under $\mathrm{N}_{2}$ atmosphere. Then the mixture was cooled down to room temperature. Only trace amount of the coupling product 3a was observed from the crude ${ }^{1} \mathrm{H}$ NMR.

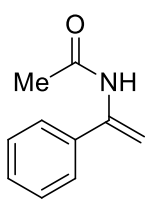

$1 \mathrm{a}$
$(0.2 \mathrm{mmol})$

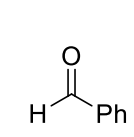

2a (2.0 equiv)

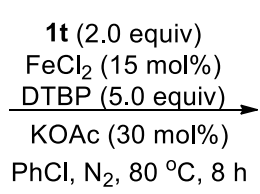

$\mathrm{PhCl}, \mathrm{N}_{2}, 80^{\circ} \mathrm{C}, 8 \mathrm{~h}$

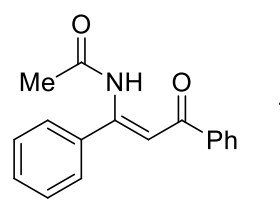

$3 a, 18 \%$

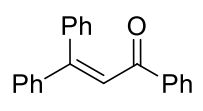

4t, $83 \%$

A $10 \mathrm{~mL}$ screw-capped Schlenk tube was charged with the enamide 1a $(32.3 \mathrm{mg}, 0.2 \mathrm{mmol}, 1.0$ equiv), $\mathrm{FeCl}_{2}$ (4.0 mg, $0.03 \mathrm{mmol}, 15 \mathrm{~mol} \%$ ), benzaldehyde $2 \mathrm{a}$ (42.5 mg, $0.4 \mathrm{mmol}, 2.0$ equiv), DTBP (146.3 mg, $1 \mathrm{mmol}, 5.0$ equiv), KOAc (5.9 mg, $0.06 \mathrm{mmol}, 0.3$ equiv), 1,1-diphenylethylene $1 \mathrm{t}$ (72.1 $\mathrm{mg}, 0.4 \mathrm{mmol}, 2.0$ equiv) in $\mathrm{PhCl}(0.8 \mathrm{~mL})$. The mixture was stirred at $80{ }^{\circ} \mathrm{C}$ in oil bath for $8 \mathrm{~h}$ under $\mathrm{N}_{2}$ atmosphere. Then the mixture was cooled down to room temperature. The resultant residue was purified by flash chromatography (PE/EA) on silica gel to afford 3a (9.6 mg, 18\% yield) and $\mathbf{4 t}$ (47.4 $\mathrm{mg}, 83 \%$ yield). The characterization of compound $\mathbf{4 t}$ is consistent with the data reported in the literature. ${ }^{9}$ 


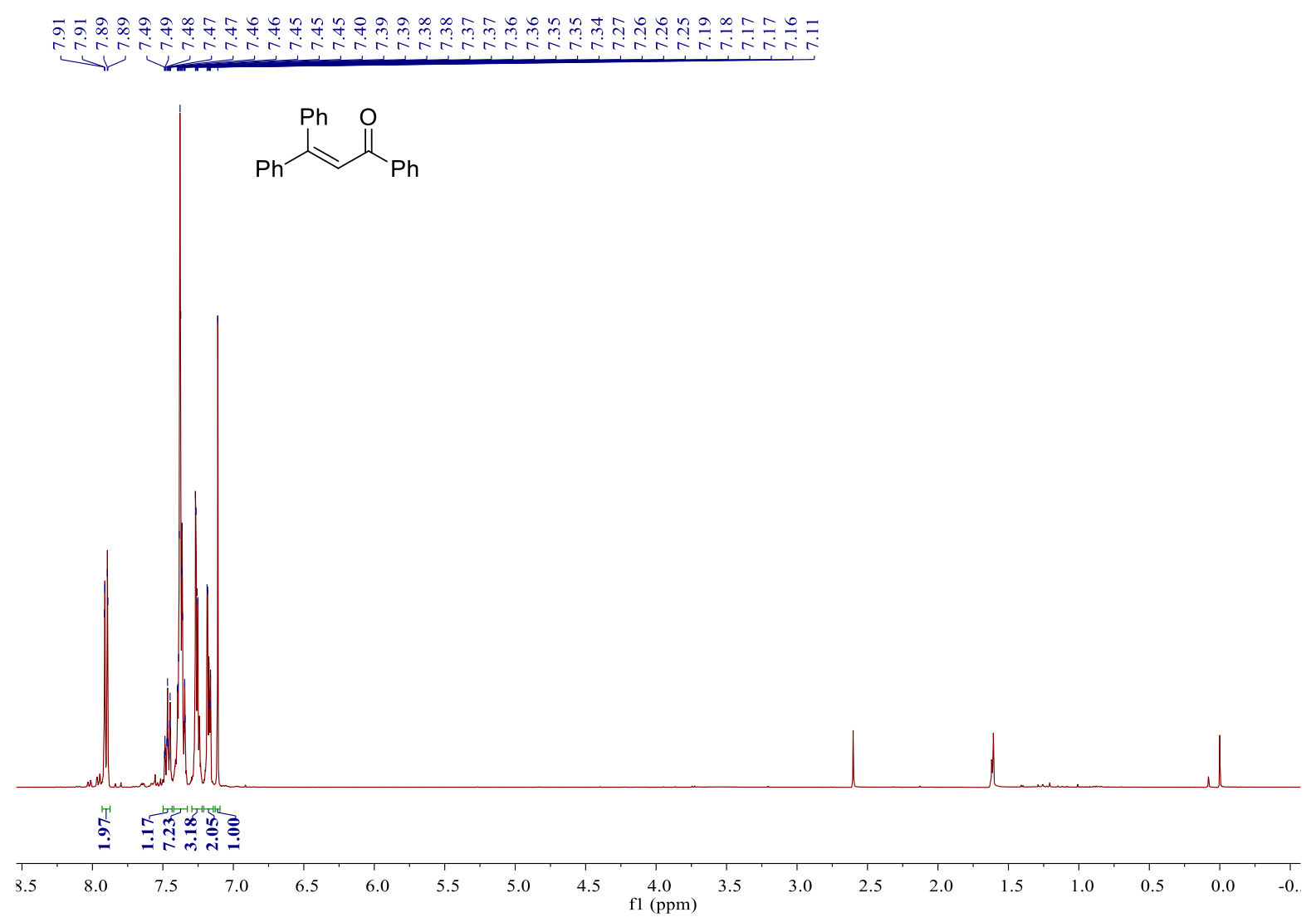

\subsubsection{Two parallel reactions}
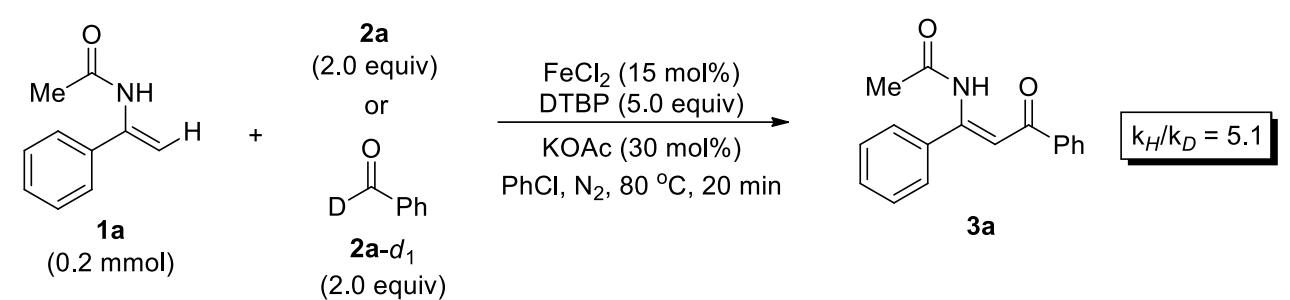

Two separate $10 \mathrm{~mL}$ screw-capped Schlenk tubes were charged with the enamide 1a (32.3 mg, $0.2 \mathrm{mmol}, 1.0$ equiv), $\mathrm{FeCl}_{2}$ (4.0 mg, $0.03 \mathrm{mmol}, 15 \mathrm{~mol} \%$ ), DTBP (146.3 mg, $1.0 \mathrm{mmol}, 5$ equiv), $2 \mathrm{a}$ (42.5 mg, $0.2 \mathrm{mmol}, 2.0$ equiv) or $\mathbf{2 a}-d_{1}\left(85.0 \mathrm{mg}, 0.2 \mathrm{mmol}, 2.0\right.$ equiv).$^{10}$ The two mixtures were stirred at $80{ }^{\circ} \mathrm{C}$ in oil bath for 20 min under $\mathrm{N}_{2}$ atmosphere. After cooling down, the two separate mixtures were purified by flash chromatography (PE /EA) on silica gel to afford the product 3a (32.2 $\mathrm{mg}$ and $6.2 \mathrm{mg}, 61 \%$ and $12 \%$ yields, respectively). 


\subsection{NMR data of the products}

(Z)-N-(3-Oxo-1,3-diphenylprop-1-en-1-yl)acetamide (3a)

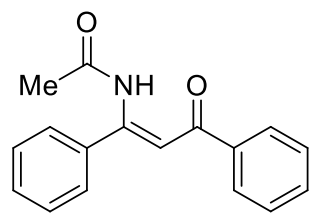

The title compound was prepared according to the typical procedure and isolated as a white solid $(40.2 \mathrm{mg}, 0.15 \mathrm{mmol}, 76 \%)$. mp: $77.8-79.2{ }^{\circ} \mathrm{C} .{ }^{1} \mathrm{H}$

NMR (400 MHz, Chloroform-d) $\delta 12.27$ (brs, 1H), 7.99 - 7.94 (m, 2H), 7.61 $7.54(\mathrm{~m}, 1 \mathrm{H}), 7.52-7.39(\mathrm{~m}, 7 \mathrm{H}), 6.33(\mathrm{~s}, 1 \mathrm{H}), 2.25(\mathrm{~s}, 3 \mathrm{H}) .{ }^{13} \mathrm{C}$ NMR $(101 \mathrm{MHz}$, Chloroform- $d) \delta$ 191.6, 168.8, 156.2, 138.5, 136.2, 132.7, 129.8, $128.6 \times 2,128.0 \times 2,127.8 \times 2,127.3 \times 2,104.7,25.1$. HRMS (ESI): $\mathrm{m} / \mathrm{z}$ calculated for $\mathrm{C}_{17} \mathrm{H}_{16} \mathrm{NO}_{2}[\mathrm{M}+\mathrm{H}]^{+}: 266.1181$, found: 266.1181 .

(Z)-N-(3-(4-Fluorophenyl)-3-oxo-1-phenylprop-1-en-1-yl)acetamide (3b)<smiles>CC(=O)N/C(=C\C(=O)c1ccc(F)cc1)c1ccccc1</smiles>

The title compound was prepared according to the typical procedure and isolated as a yellow solid (42.3 mg, $0.15 \mathrm{mmol}, 75 \%)$. $\mathrm{mp}: 87.0-88.6^{\circ} \mathrm{C} .{ }^{1} \mathrm{H}$

NMR (400 MHz, Chloroform- $d$ ) $\delta 12.23$ (brs, 1H), $8.03-7.96$ (m, 2H), 7.50 $-7.38(\mathrm{~m}, 5 \mathrm{H}), 7.19-7.10(\mathrm{~m}, 2 \mathrm{H}), 6.27$ (s, 1H), $2.25(\mathrm{~s}, 3 \mathrm{H}) .{ }^{13} \mathrm{C}$ NMR (101 MHz, Chloroform- $\left.d\right) \delta$ 190.0, 168.8, 165.5 (d, $J=254.4 \mathrm{~Hz}), 156.5,136.1,134.8$ (d, $J=3.3 \mathrm{~Hz}), 130.3$ (d, $J=9.3 \mathrm{~Hz}), 129.8$, $128.1 \times 2,127.3 \times 2,115.7(\mathrm{~d}, J=22.0 \mathrm{~Hz}), 104.3,25.1 .{ }^{19} \mathrm{~F}$ NMR $(376 \mathrm{MHz}$, Chloroform- $d) \delta$ -105.51. HRMS (ESI): $\mathrm{m} / \mathrm{z}$ calculated for $\mathrm{C}_{17} \mathrm{H}_{15} \mathrm{FNO}_{2}[\mathrm{M}+\mathrm{H}]^{+}: 284.1087$, found: 284.1083.

\section{(Z)- $N$-(3-(3-Chlorophenyl)-3-oxo-1-phenylprop-1-en-1-yl)acetamide (3c)}<smiles>CC(=O)N/C(=C\C(=O)c1cccc(Cl)c1)c1ccccc1</smiles>

The title compound was prepared according to the typical procedure and isolated as a colorless oil (36.2 mg, $0.12 \mathrm{mmol}, 60 \%) .{ }^{1} \mathrm{H}$ NMR (400 MHz, Chloroform- $d$ ) $\delta 12.21$ (brs, 1H), 7.93 (t, $J=1.9 \mathrm{~Hz}, 1 \mathrm{H}), 7.83$ (dt, $J=7.8,1.3$ $\mathrm{Hz}, 1 \mathrm{H}), 7.53(\mathrm{ddd}, J=8.0,2.1,1.1 \mathrm{~Hz}, 1 \mathrm{H}), 7.49-7.39(\mathrm{~m}, 6 \mathrm{H}), 6.24(\mathrm{~s}, 1 \mathrm{H})$,

2.25 (s, 3H). ${ }^{13} \mathrm{C}$ NMR (101 MHz, Chloroform- $d$ ) $\delta$ 190.0, 168.8, 157.2, 140.2, 135.9, 134.9, 132.6, $130.0,130.0,128.1 \times 2,127.9,127.4 \times 2,125.8,104.2,25.1$. HRMS $(E S I): \mathrm{m} / \mathrm{z}$ calculated for $\mathrm{C}_{17} \mathrm{H}_{15}{ }^{35} \mathrm{ClNO}_{2}[\mathrm{M}+\mathrm{H}]^{+}:$300.0791, found: 300.0787 . 


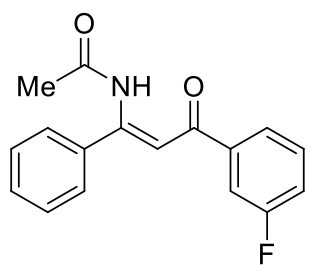

The title compound was prepared according to the typical procedure and isolated as a yellow solid (38.8 mg, $0.138 \mathrm{mmol}, 69 \%)$. mp: $92.9-94.3{ }^{\circ} \mathrm{C} .{ }^{1} \mathrm{H}$ NMR (400 MHz, Chloroform-d) $\delta 12.21$ (brs, 1H), 7.74 (dt, $J=7.8,1.2 \mathrm{~Hz}$, $1 \mathrm{H}), 7.65(\mathrm{ddd}, J=9.6,2.6,1.6 \mathrm{~Hz}, 1 \mathrm{H}), 7.50-7.39(\mathrm{~m}, 6 \mathrm{H}), 7.30-7.23$ (m, $1 \mathrm{H}), 6.26(\mathrm{~s}, 1 \mathrm{H}), 2.26(\mathrm{~s}, 3 \mathrm{H}) .{ }^{13} \mathrm{C}$ NMR $(101 \mathrm{MHz}$, Chloroform- $d) \delta 190.1\left(\mathrm{~d}, J_{\mathrm{C}-\mathrm{F}}=2.4 \mathrm{~Hz}\right), 168.8$, $162.9\left(\mathrm{~d}, J_{\mathrm{C}-\mathrm{F}}=247.7 \mathrm{~Hz}\right), 157.0,140.7\left(\mathrm{~d}, J_{\mathrm{C}-\mathrm{F}}=6.3 \mathrm{~Hz}\right), 136.0,130.3,130.0,128.1 \times 2,127.4 \times 2$, $123.4\left(\mathrm{~d}, J_{\mathrm{C}-\mathrm{F}}=3.3 \mathrm{~Hz}\right), 119.7\left(\mathrm{~d}, J_{\mathrm{C}-\mathrm{F}}=21.7 \mathrm{~Hz}\right), 114.7\left(\mathrm{~d}, J_{\mathrm{C}-\mathrm{F}}=22.5 \mathrm{~Hz}\right), 104.3,25.1 .{ }^{19} \mathrm{~F} \mathrm{NMR}$ (376 MHz, Chloroform- $d$ ) $\delta$-111.61. HRMS (ESI): $\mathrm{m} / \mathrm{z}$ calculated for $\mathrm{C}_{17} \mathrm{H}_{15} \mathrm{FNO}_{2}[\mathrm{M}+\mathrm{H}]^{+}$: 284.1087, found: 284.1084 .

\section{(Z)- $N$-(3-(4-Bromophenyl)-3-oxo-1-phenylprop-1-en-1-yl)acetamide (3e)}<smiles>CC(=O)N/C(=C\C(=O)c1ccc(Br)cc1)c1ccccc1</smiles>

The title compound was prepared according to the typical procedure and isolated as a yellow solid $(49.2 \mathrm{mg}, 0.142 \mathrm{mmol}, 71 \%) . \mathrm{mp}: 102.1-$ 104.6 ${ }^{\circ} \mathrm{C} .{ }^{1} \mathrm{H}$ NMR (400 MHz, Chloroform- $d$ ) $\delta 12.23$ (brs, 1H), 7.83 (d, $J=$ $8.6 \mathrm{~Hz}, 2 \mathrm{H}), 7.61(\mathrm{~d}, J=8.6 \mathrm{~Hz}, 2 \mathrm{H}), 7.48-7.39(\mathrm{~m}, 5 \mathrm{H}), 6.25(\mathrm{~s}, 1 \mathrm{H}), 2.25(\mathrm{~s}, 3 \mathrm{H}) .{ }^{13} \mathrm{C} \mathrm{NMR}(101$ MHz, Chloroform- $d$ ) $\delta 190.3,168.8,156.8,137.3,136.0,131.9 \times 2,129.9,129.3 \times 2,128.1 \times 2$, 127.8, $127.3 \times 2,104.1,25.1$. HRMS (ESI): $\mathrm{m} / \mathrm{z}$ calculated for $\mathrm{C}_{17} \mathrm{H}_{15}{ }^{79} \mathrm{BrNO}_{2}[\mathrm{M}+\mathrm{H}]^{+}: 344.0286$, found: 344.0279 .

\section{(Z)-N-(3-(3,4-Difluorophenyl)-3-oxo-1-phenylprop-1-en-1-yl)acetamide (3f)}<smiles>CC(=O)N/C(=C\C(=O)c1ccc(F)c(F)c1)c1ccccc1</smiles>
The title compound was prepared according to the typical procedure and isolated as a yellow solid $(46.7 \mathrm{mg}, 0.155 \mathrm{mmol}, 78 \%)$. mp: $83.8-85.3{ }^{\circ} \mathrm{C}$. ${ }^{1} \mathrm{H}$ NMR (400 MHz, Chloroform- $d$ ) $\delta 12.18$ (brs, 1H), 7.81 (ddd, $J=10.9$, 7.6, 2.2 Hz, 1H), $7.76-7.72(\mathrm{~m}, 1 \mathrm{H}), 7.50-7.39(\mathrm{~m}, 5 \mathrm{H}), 7.30-7.22(\mathrm{~m}, 1 \mathrm{H}), 6.20(\mathrm{~s}, 1 \mathrm{H}), 2.25(\mathrm{~s}$, 3H). ${ }^{13} \mathrm{C}$ NMR (101 MHz, Chloroform- $d$ ) $\delta 188.7\left(\mathrm{~d}, J_{\mathrm{C}-\mathrm{F}}=2.5 \mathrm{~Hz}\right), 168.8,157.3,153.3\left(\mathrm{dd}, J_{\mathrm{C}-\mathrm{F}}=\right.$ $256.7,13.0 \mathrm{~Hz}), 150.4\left(\mathrm{dd}, J_{\mathrm{C}-\mathrm{F}}=250.8,13.0 \mathrm{~Hz}\right), 135.9,135.6\left(\mathrm{dd}, J_{\mathrm{C}-\mathrm{F}}=4.9,3.5 \mathrm{~Hz}\right), 130.0,128.15$ $\times 2,127.3 \times 2,124.6\left(\mathrm{dd}, J_{\mathrm{C}-\mathrm{F}}=7.6,3.6 \mathrm{~Hz}\right), 117.5\left(\mathrm{~d}, J_{\mathrm{C}-\mathrm{F}}=17.8 \mathrm{~Hz}\right), 117.2\left(\mathrm{dd}, J_{\mathrm{C}-\mathrm{F}}=18.0,2.0 \mathrm{~Hz}\right)$, 103.7, 25.1. ${ }^{19} \mathrm{~F}$ NMR (376 MHz, Chloroform- $d$ ) $\delta-130.17,-135.78$. HRMS (ESI): m/z calculated for $\mathrm{C}_{17} \mathrm{H}_{14} \mathrm{~F}_{2} \mathrm{NO}_{2}[\mathrm{M}+\mathrm{H}]^{+}:$302.0993, found: 302.0990. 
(Z)- $N$-(3-(2-Chlorophenyl)-3-oxo-1-phenylprop-1-en-1-yl)acetamide (3g)

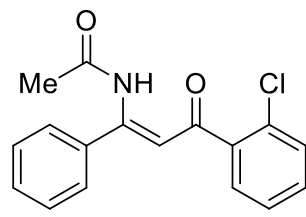

The title compound was prepared according to a modified procedure $(12 \mathrm{~h}$, with $25 \mathrm{~mol} \%$ catalyst) and isolated as a yellow solid (24.5 $\mathrm{mg}, 0.082 \mathrm{mmol}, 41 \%)$. mp: $98.8-99.3{ }^{\circ} \mathrm{C} .{ }^{1} \mathrm{H}$ NMR (400 MHz, Chloroform- $d$ ) $\delta 11.96$ (brs, $\left.1 \mathrm{H}\right), 7.56$ - $7.53(\mathrm{~m}, 1 \mathrm{H}), 7.48-7.41(\mathrm{~m}, 4 \mathrm{H}), 7.40-7.38(\mathrm{~m}, 3 \mathrm{H}), 7.37-7.33(\mathrm{~m}, 1 \mathrm{H}), 6.08(\mathrm{~s}, 1 \mathrm{H}), 2.26(\mathrm{~s}$, 3H). ${ }^{13} \mathrm{C}$ NMR (101 MHz, Chloroform- $d$ ) $\delta$ 193.3, 169.0, 155.9, 139.7, 135.6, 131.7, 131.1, 130.5, $130.0,129.5,128.1 \times 2,127.5 \times 2,127.0,108.7,25.2$. HRMS $(\mathrm{ESI}): \mathrm{m} / \mathrm{z}$ calculated for $\mathrm{C}_{17} \mathrm{H}_{15} \mathrm{ClNO}_{2}$ $[\mathrm{M}+\mathrm{H}]^{+}:$300.0791, found: 300.0786 .

\section{(Z)-N-(3-(4-Cyanophenyl)-3-oxo-1-phenylprop-1-en-1-yl)acetamide (3h)}

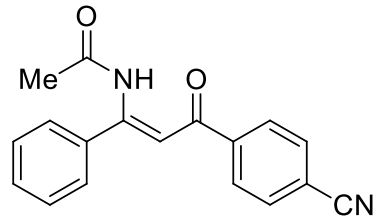

The title compound was prepared according to the typical procedure and isolated as a yellow oil (38.2 mg, $0.132 \mathrm{mmol}, 66 \%) .{ }^{1} \mathrm{H}$ NMR (400 MHz,

Chloroform- $d$ ) $\delta 12.20$ (brs, $1 \mathrm{H}), 8.04(\mathrm{~d}, J=8.5 \mathrm{~Hz}, 2 \mathrm{H}), 7.78$ (d, $J=8.4$ $\mathrm{Hz}, 2 \mathrm{H}), 7.51-7.38(\mathrm{~m}, 5 \mathrm{H}), 6.25(\mathrm{~s}, 1 \mathrm{H}), 2.26(\mathrm{~s}, 3 \mathrm{H}) .{ }^{13} \mathrm{C}$ NMR (101 MHz, Chloroform- $d$ ) $\delta$ 189.6, $168.8,158.1,141.9,135.7,132.5 \times 2,130.2,128.2 \times 4,127.4 \times 2,118.0,115.8,103.9,25.2$. HRMS (ESI): $\mathrm{m} / \mathrm{z}$ calculated for $\mathrm{C}_{18} \mathrm{H}_{15} \mathrm{~N}_{2} \mathrm{O}_{2}[\mathrm{M}+\mathrm{H}]^{+}: 291.1134$, found: 291,1127 .

\section{(Z)-N-(3-Oxo-1-phenyl-3-(4-(trifluoromethyl)phenyl)prop-1-en-1-yl)acetamide (3i)}

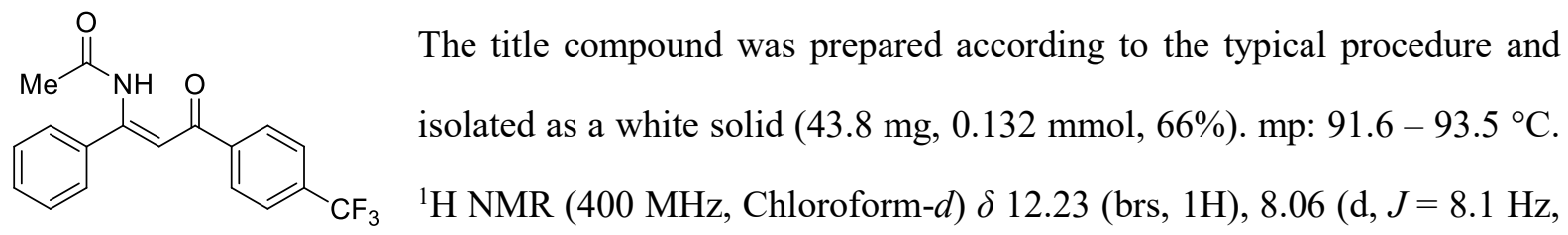
2H), $7.74(\mathrm{~d}, J=8.2 \mathrm{~Hz}, 2 \mathrm{H}), 7.51-7.38(\mathrm{~m}, 5 \mathrm{H}), 6.29(\mathrm{~s}, 1 \mathrm{H}), 2.26(\mathrm{~s}, 3 \mathrm{H}) .{ }^{13} \mathrm{C}$ NMR $(101 \mathrm{MHz}$, Chloroform- $d$ ) $\delta 190.3,168.8,157.6,141.5,135.9,133.9\left(\mathrm{~d}, J_{\mathrm{C}-\mathrm{F}}=32.5 \mathrm{~Hz}\right), 130.1,128.2 \times 2,128.1$ $\times 2,127.4 \times 2,125.7\left(\mathrm{q}, J_{\mathrm{C}-\mathrm{F}}=4.1 \mathrm{~Hz}\right), 123.6\left(\mathrm{~d}, J_{\mathrm{C}-\mathrm{F}}=272.6 \mathrm{~Hz}\right), 104.2,25.1 .{ }^{19} \mathrm{~F} \mathrm{NMR}(376 \mathrm{MHz}$, Chloroform- $d$ ) $\delta-62.89$. HRMS (ESI): $\mathrm{m} / \mathrm{z}$ calculated for $\mathrm{C}_{18} \mathrm{H}_{15} \mathrm{~F}_{3} \mathrm{NO}_{2}[\mathrm{M}+\mathrm{H}]^{+}: 334.1055$, found: 334.1050.

(Z)-Methyl-4-(3-acetamido-3-phenylacryloyl)benzoate (3j) 


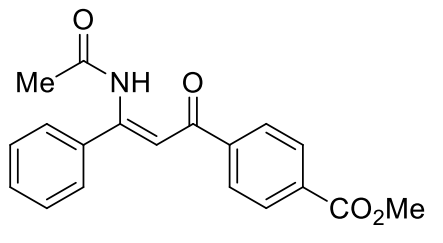

The title compound was prepared according to the typical procedure and isolated as a white solid (39.4 mg, $0.122 \mathrm{mmol}, 61 \%)$. mp: $156.8-$ $159.2{ }^{\circ} \mathrm{C} .{ }^{1} \mathrm{H}$ NMR (400 MHz, Chloroform-d) $\delta 12.24$ (brs, $\left.1 \mathrm{H}\right), 8.13$ (d, $J=8.4 \mathrm{~Hz}, 2 \mathrm{H}), 8.00(\mathrm{~d}, J=8.5 \mathrm{~Hz}, 2 \mathrm{H}), 7.50-7.38(\mathrm{~m}, 5 \mathrm{H}), 6.31(\mathrm{~s}, 1 \mathrm{H}), 3.95(\mathrm{~s}, 3 \mathrm{H}), 2.25(\mathrm{~s}, 3 \mathrm{H})$. ${ }^{13} \mathrm{C}$ NMR (101 MHz, Chloroform- $d$ ) $\delta$ 190.7, 168.8, 166.2, 157.2, 142.0, 135.9, 133.4, 130.0, $129.8 \times$ $2,128.1 \times 2,127.7 \times 2,127.4 \times 2,104.5,52.4,25.1$. HRMS $(E S I): m / z$ calculated for $\mathrm{C}_{19} \mathrm{H}_{18} \mathrm{NO}_{4}[\mathrm{M}$ $+\mathrm{H}]^{+}:$324.1236, found: 324.1232 .

\section{(Z)-N-(3-Oxo-1-phenyl-3-(m-tolyl)prop-1-en-1-yl)acetamide (3k)}<smiles>CC(=O)N/C(=C\C(=O)c1cccc(C)c1)c1ccccc1</smiles>

The title compound was prepared according to the typical procedure and isolated as a yellow oil (30.0 mg, $0.107 \mathrm{mmol}, 54 \%)$. ${ }^{1} \mathrm{H}$ NMR (400 MHz, Chloroform- $d$ ) $\delta 12.29$ (brs, $1 \mathrm{H}), 7.77$ (d, $J=6.5 \mathrm{~Hz}, 2 \mathrm{H}), 7.52-7.33$ (m, 7H), $6.32(\mathrm{~s}, 1 \mathrm{H}), 2.43(\mathrm{~s}, 3 \mathrm{H}), 2.25$ (s, 3H). ${ }^{13} \mathrm{C}$ NMR (101 MHz, Chloroform- $d$ ) $\delta$ 191.9, 168.9, $156.1,138.6,138.5,136.2,133.5,129.8,128.5,128.4,128.1 \times 2,127.4 \times 2,125.0,104.9,25.1,21.4$. HRMS (ESI): $\mathrm{m} / \mathrm{z}$ calculated for $\mathrm{C}_{18} \mathrm{H}_{18} \mathrm{NO}_{2}[\mathrm{M}+\mathrm{H}]^{+}: 280.1338$, found: 280.1336 .

\section{(Z)-N-(3-Oxo-1-phenyl-3-(p-tolyl)prop-1-en-1-yl)acetamide (3I)}

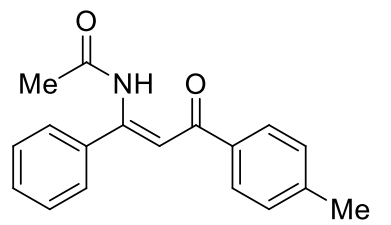

The title compound was prepared according to the typical procedure and isolated as a white solid (39.5 $\mathrm{mg}, 0.116 \mathrm{mmol}, 58 \%)$. $\mathrm{mp}: 116.1-$ $118.1^{\circ} \mathrm{C} .{ }^{1} \mathrm{H}$ NMR (400 MHz, Chloroform-d) $\delta 12.30$ (brs, $\left.1 \mathrm{H}\right), 7.88$ (d, $J=$ $8.2 \mathrm{~Hz}, 2 \mathrm{H}), 7.50-7.38(\mathrm{~m}, 5 \mathrm{H}), 7.28(\mathrm{~d}, J=8.4 \mathrm{~Hz}, 2 \mathrm{H}), 6.32(\mathrm{~s}, 1 \mathrm{H}), 2.43(\mathrm{~s}, 3 \mathrm{H}), 2.25(\mathrm{~s}, 3 \mathrm{H}) .{ }^{13} \mathrm{C}$ NMR (101 MHz, Chloroform-d) $\delta$ 191.3, 168.9, 155.8, 143.6, 136.3, 135.9, 129.7, $129.4 \times 2,128.0 \times$ 2, $127.9 \times 2,127.3 \times 2,104.8,25.1,21.6 . \mathrm{HRMS}(\mathrm{ESI}): \mathrm{m} / \mathrm{z}$ calculated for $\mathrm{C}_{18} \mathrm{H}_{18} \mathrm{NO}_{2}[\mathrm{M}+\mathrm{H}]^{+}:$ 280.1338 , found: 280.1335 .

\section{(Z)-N-(3-([1,1'-Biphenyl]-4-yl)-3-oxo-1-phenylprop-1-en-1-yl)acetamide (3m)}

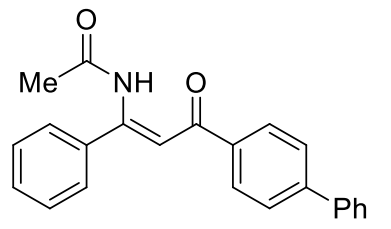

The title compound was prepared according to the typical procedure and isolated as a yellow oil (43.0 mg, $0.126 \mathrm{mmol}, 63 \%) .{ }^{1} \mathrm{H}$ NMR $(400 \mathrm{MHz}$,

Chloroform- $d$ ) $\delta 12.33$ (brs, 1H), $8.06(\mathrm{~d}, J=8.4 \mathrm{~Hz}, 2 \mathrm{H}), 7.72(\mathrm{~d}, J=8.5$

$\mathrm{Hz}, 2 \mathrm{H}), 7.68-7.62(\mathrm{~m}, 2 \mathrm{H}), 7.53-7.38(\mathrm{~m}, 8 \mathrm{H}), 6.38(\mathrm{~s}, 1 \mathrm{H}), 2.27(\mathrm{~s}, 3 \mathrm{H}) .{ }^{13} \mathrm{C}$ NMR $(101 \mathrm{MHz}$, 
Chloroform- $d$ ) $\delta 191.1,168.9,156.3,145.5,139.8,137.3,136.3,129.8,128.9 \times 2,128.4 \times 2,128.2$, $128.1 \times 2,127.4 \times 2,127.3 \times 2,127.2 \times 2,104.8,25.1$. HRMS $(E S I): \mathrm{m} / \mathrm{z}$ calculated for $\mathrm{C}_{23} \mathrm{H}_{20} \mathrm{NO}_{2}$ $[\mathrm{M}+\mathrm{H}]^{+}:$342.1494, found: 342.1490 .

(Z)-N-(3-(4-Methoxyphenyl)-3-oxo-1-phenylprop-1-en-1-yl)acetamide (3n)

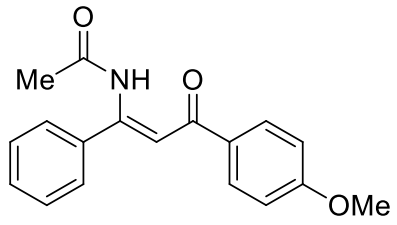

The title compound was prepared according to a modified procedure $(12 \mathrm{~h}$, with $25 \mathrm{~mol} \%$ catalyst) and isolated as a white solid $(33.2 \mathrm{mg}, 0.112$ mmol, 56\%). mp: $133.0-134.2{ }^{\circ} \mathrm{C} .{ }^{1} \mathrm{H}$ NMR (400 MHz, Chloroform- $d$ ) $\delta$ 12.30 (brs, 1H), 7.97 (d, $J=8.9 \mathrm{~Hz}, 2 \mathrm{H}), 7.52-7.36(\mathrm{~m}, 5 \mathrm{H}), 6.96(\mathrm{~d}, J=8.9 \mathrm{~Hz}, 2 \mathrm{H}), 6.30(\mathrm{~s}, 1 \mathrm{H})$, $3.88(\mathrm{~s}, 3 \mathrm{H}), 2.24(\mathrm{~s}, 3 \mathrm{H}) .{ }^{13} \mathrm{C}$ NMR (101 MHz, Chloroform-d) $\delta$ 190.3, 168.9, 163.4, 155.4, 136.4, $131.3,130.1 \times 2,129.6,128.0 \times 2,127.3 \times 2,113.9 \times 2,104.7,55.5,25.1$. HRMS $(E S I): \mathrm{m} / \mathrm{z}$ calculated for $\mathrm{C}_{18} \mathrm{H}_{18} \mathrm{NO}_{3}[\mathrm{M}+\mathrm{H}]^{+}: 296.1287$, found: 296.1282 .

(Z)-N-(3-Oxo-1-phenyl-3-(4-(trifluoromethoxy)phenyl)prop-1-en-1-yl)acetamide (3o)

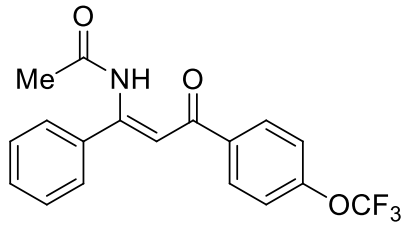

The title compound was prepared according to the typical procedure and isolated as a yellow oil (46.8 mg, $0.134 \mathrm{mmol}, 67 \%) .{ }^{1} \mathrm{H}$ NMR $(400 \mathrm{MHz}$, Chloroform- $d$ ) $\delta 12.23$ (brs, 1H), $8.06-7.98(\mathrm{~m}, 2 \mathrm{H}), 7.51-7.38(\mathrm{~m}$, 5H), $7.31(\mathrm{~d}, J=8.8 \mathrm{~Hz}, 2 \mathrm{H}), 6.27(\mathrm{~s}, 1 \mathrm{H}), 2.25(\mathrm{~s}, 3 \mathrm{H}) .{ }^{13} \mathrm{C}$ NMR (101 MHz, Chloroform- $\left.d\right) \delta$ 189.9, $168.8,157.0,152.3(\mathrm{~d}, J=2.1 \mathrm{~Hz}) ., 136.4\left(\mathrm{~d}, J_{\mathrm{C}-\mathrm{F}}=81.0 \mathrm{~Hz}\right), 130.0,129.7 \times 2,128.1 \times 2,127.3 \times 2$, 121.5, $120.4\left(\mathrm{q}, J_{\mathrm{C}-\mathrm{F}}=1.2 \mathrm{~Hz}\right), 119.0,104.2,25.1 .{ }^{19} \mathrm{~F}$ NMR $(376 \mathrm{MHz}$, Chloroform- $d$ ) $\delta-57.48$. HRMS (ESI): $\mathrm{m} / \mathrm{z}$ calculated for $\mathrm{C}_{18} \mathrm{H}_{15} \mathrm{~F}_{3} \mathrm{NO}_{3}[\mathrm{M}+\mathrm{H}]^{+}: 350.1004$, found: 350.0997 .

\section{(Z)-N-(3-(3-Bromo-4-methoxyphenyl)-3-oxo-1-phenylprop-1-en-1-yl)acetamide (3p)}

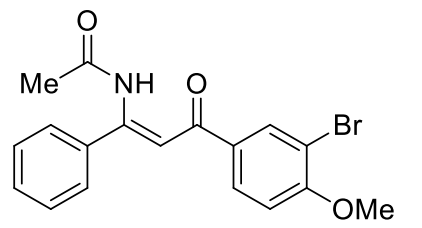

The title compound was prepared according to a modified procedure $(5 \mathrm{~h})$ and isolated as a white solid (49.4 mg, $0.132 \mathrm{mmol}, 66 \%)$ mp: $149.8-$ $152.5^{\circ} \mathrm{C} .{ }^{1} \mathrm{H}$ NMR $(400 \mathrm{MHz}$, Chloroform- $d$ ) $\delta 12.25$ (s, 1H), 8.19 (d, $J=$ $2.2 \mathrm{~Hz}, 1 \mathrm{H}), 7.94(\mathrm{dd}, J=8.7,2.2 \mathrm{~Hz}, 1 \mathrm{H}), 7.50-7.38(\mathrm{~m}, 5 \mathrm{H}), 6.95(\mathrm{~d}, J=8.7 \mathrm{~Hz}, 1 \mathrm{H}), 6.23(\mathrm{~s}, 1 \mathrm{H})$, 3.97 (s, 3H), 2.24 (s, 3H). ${ }^{13} \mathrm{C}$ NMR (101 MHz, Chloroform-d) $\delta 189.0,169.0,159.4,156.3,136.2$, 133.3, $132.4,129.8,129.0,128.1 \times 2,127.4 \times 2,112.0,111.2,104.1,56.5,25.1$. HRMS $(E S I): \mathrm{m} / \mathrm{z}$ calculated for $\mathrm{C}_{18} \mathrm{H}_{17}{ }^{79} \mathrm{BrNO}_{3}[\mathrm{M}+\mathrm{H}]^{+}: 374.0392$, found: 374.0385 . 
(Z)-N-(3-Oxo-1-phenyl-3-(4-(4,4,5,5-tetramethyl-1,3,2-dioxaborolan-2-yl)phenyl)prop-1-en-1-yl) acetamide (3q)

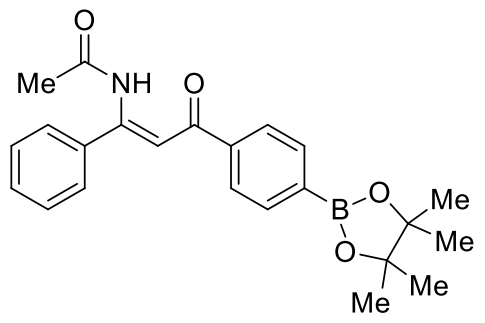

The title compound was prepared according to a modified procedure (5 h, with $25 \mathrm{~mol} \%$ catalyst) and isolated as a yellow oil $(53.2 \mathrm{mg}$, $0.136 \mathrm{mmol}, 68 \%) .{ }^{1} \mathrm{H}$ NMR (401 MHz, Chloroform- $d$ ) $\delta 12.30$ (brs, 1H), $7.97-7.89(\mathrm{~m}, 4 \mathrm{H}), 7.50-7.40(\mathrm{~m}, 5 \mathrm{H}), 6.33(\mathrm{~s}, 1 \mathrm{H}), 2.25(\mathrm{~s}$, 3H), 1.37 (s, 12H). ${ }^{13} \mathrm{C}$ NMR (101 MHz, Chloroform- $d$ ) $\delta 191.8$, $168.9,156.5,140.6,136.1,135.2,135.0 \times 2,129.8,128.1 \times 2,127.4 \times 2,126.8 \times 2,104.8,84.2 \times 2$, 25.1, $24.8 \times 4$. HRMS (ESI): $\mathrm{m} / \mathrm{z}$ calculated for $\mathrm{C}_{23} \mathrm{H}_{27} \mathrm{BNO}_{4}[\mathrm{M}+\mathrm{H}]^{+}:$392.2033, found: 392.2028.

(Z)- $N$-(3-Oxo-1-phenyl-3-(4-((trimethylsilyl)ethynyl)phenyl)prop-1-en-1-yl)acetamide (3r)

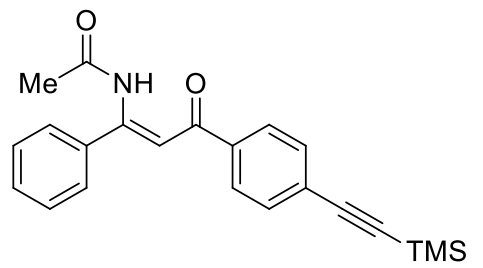

The title compound was prepared according to a modified procedure (12 h, with $25 \mathrm{~mol} \%$ catalyst) and isolated as a yellow oil (40.4 mg, $0.112 \mathrm{mmol}, 56 \%) .{ }^{1} \mathrm{H}$ NMR (400 MHz, Chloroform- $d$ ) $\delta 12.27$ (brs, $1 \mathrm{H}), 7.90(\mathrm{~d}, J=8.5 \mathrm{~Hz}, 2 \mathrm{H}), 7.56(\mathrm{~d}, J=8.4 \mathrm{~Hz}, 2 \mathrm{H}), 7.48-7.40$ (m, 5H), $6.29(\mathrm{~s}, 1 \mathrm{H}), 2.25(\mathrm{~s}, 3 \mathrm{H}), 0.27(\mathrm{~s}, 9 \mathrm{H}) .{ }^{13} \mathrm{C}$ NMR (101 MHz, Chloroform- $\left.d\right) \delta$ 190.6, 168.9, $156.7,137.9,136.1,132.1 \times 2,129.9,128.1 \times 2,127.6 \times 2,127.6,127.4 \times 2,104.5,104.0,98.2,25.1$, $-0.2 \times 3$. HRMS (ESI): $\mathrm{m} / \mathrm{z}$ calculated for $\mathrm{C}_{22} \mathrm{H}_{24} \mathrm{NO}_{2} \mathrm{Si}[\mathrm{M}+\mathrm{H}]^{+}: 362.1576$, found: 362.1570 .

\section{(Z)-N-(3-(Naphthalen-2-yl)-3-oxo-1-phenylprop-1-en-1-yl)acetamide (3s)}

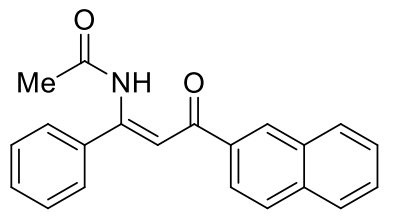

The title compound was prepared according to the typical procedure and isolated as a yellow solid (39.7 mg, $0.126 \mathrm{mmol}, 63 \%)$. mp: $114.9-$ $118.4{ }^{\circ} \mathrm{C} .{ }^{1} \mathrm{H}$ NMR (400 MHz, Chloroform- $d$ ) $\delta 12.37$ (brs, $1 \mathrm{H}$ ), 8.49 (s, 1H), $8.06(\mathrm{dd}, J=8.6,1.8 \mathrm{~Hz}, 1 \mathrm{H}), 7.93(\mathrm{~m}, 3 \mathrm{H}), 7.65-7.50(\mathrm{~m}, 4 \mathrm{H}), 7.50-7.41(\mathrm{~m}, 3 \mathrm{H}), 6.49$ (s, 1H), 2.28 (s, 3H). ${ }^{13} \mathrm{C}$ NMR (101 MHz, Chloroform- $d$ ) $\delta$ 191.4, 168.9, 156.2, 136.3, 135.9, 135.4, $132.5,129.8,129.5,129.2,128.6,128.4,128.1 \times 2,127.7,127.4 \times 2,126.8,123.8,104.8,25.1$. HRMS (ESI): $\mathrm{m} / \mathrm{z}$ calculated for $\mathrm{C}_{21} \mathrm{H}_{18} \mathrm{NO}_{2}[\mathrm{M}+\mathrm{H}]^{+}: 316.1338$, found: 316.1334 .

(Z)- $N$-(3-Oxo-1-phenyl-3-(thiophen-2-yl)prop-1-en-1-yl)acetamide (3t) 


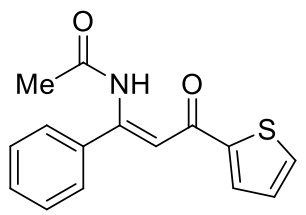

The title compound was prepared according to the typical procedure and isolated as a yellow solid (39.1 mg, $0.144 \mathrm{mmol}, 72 \%)$. mp: $87.8-89.4{ }^{\circ} \mathrm{C} .{ }^{1} \mathrm{H}$ NMR (400 MHz, Chloroform- $d$ ) $\delta 12.07$ (brs, 1H), 7.74 (dd, $J=3.8,1.1 \mathrm{~Hz}, 1 \mathrm{H}), 7.66$ $(\mathrm{dd}, J=4.9,1.0 \mathrm{~Hz}, 1 \mathrm{H}), 7.49-7.38(\mathrm{~m}, 5 \mathrm{H}), 7.15(\mathrm{dd}, J=4.9,3.9 \mathrm{~Hz}, 1 \mathrm{H}), 6.17(\mathrm{~s}, 1 \mathrm{H}), 2.22(\mathrm{~s}$, 3H). ${ }^{13} \mathrm{C}$ NMR (101 MHz, Chloroform- $d$ ) $\delta$ 183.9, 168.7, 155.9, 145.7, 135.9, 133.8, 130.9, 129.8, $128.4,128.1 \times 2,127.3 \times 2,104.7,25.0$. HRMS $\left(\right.$ ESI): $\mathrm{m} / \mathrm{z}$ calculated for $\mathrm{C}_{15} \mathrm{H}_{14} \mathrm{NO}_{2} \mathrm{~S}[\mathrm{M}+\mathrm{H}]^{+}:$ 272.0745, found: 272.0741 .

\section{(Z)-N-(3-(Furan-2-yl)-3-oxo-1-phenylprop-1-en-1-yl)acetamide (3u)}

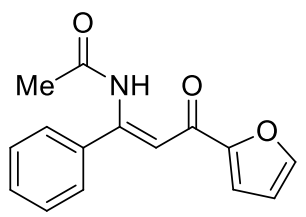

The title compound was prepared according to a modified procedure $(12 \mathrm{~h}$, with $25 \mathrm{~mol} \%$ catalyst) and isolated as a yellow oil $(28.1 \mathrm{mg}, 0.110 \mathrm{mmol}, 55 \%) .{ }^{1} \mathrm{H}$ NMR (400 MHz, Chloroform- $d$ ) $\delta 12.06$ (brs, 1H), 7.61 (dd, $J=1.7,0.7 \mathrm{~Hz}$, 1H), $7.48-7.37(\mathrm{~m}, 5 \mathrm{H}), 7.23(\mathrm{dd}, J=3.6,0.8 \mathrm{~Hz}, 1 \mathrm{H}), 6.57$ (dd, $J=3.5,1.7 \mathrm{~Hz}, 1 \mathrm{H}), 6.23(\mathrm{~s}, 1 \mathrm{H})$, $2.22(\mathrm{~s}, 3 \mathrm{H}) .{ }^{13} \mathrm{C}$ NMR $(101 \mathrm{MHz}$, Chloroform- $d$ ) $\delta 179.8,168.8,156.3,153.4,146.4,135.9,129.9$, $128.0 \times 2,127.4 \times 2,116.7,112.6,104.4,25.1 . \mathrm{HRMS}(\mathrm{ESI}): \mathrm{m} / \mathrm{z}$ calculated for $\mathrm{C}_{15} \mathrm{H}_{14} \mathrm{NO}_{3}[\mathrm{M}+\mathrm{H}]^{+}:$ 256.0974, found: 256.0966 .

\section{(Z)- $N$-(3-(1-Methyl-1H-indol-2-yl)-3-oxo-1-phenylprop-1-en-1-yl)acetamide (3v)}

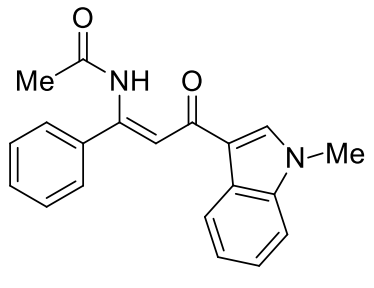

The title compound was prepared according to the typical procedure and isolated as a yellow oil (38.8 mg, $0.122 \mathrm{mmol}, 61 \%) .{ }^{1} \mathrm{H} \mathrm{NMR}(400 \mathrm{MHz}$, Chloroform- $d$ ) $\delta 12.37$ (brs, 1H), $8.50-8.43(\mathrm{~m}, 1 \mathrm{H}), 7.76(\mathrm{~s}, 1 \mathrm{H}), 7.50-$ $7.44(\mathrm{~m}, 2 \mathrm{H}), 7.44-7.39(\mathrm{~m}, 3 \mathrm{H}), 7.38-7.33(\mathrm{~m}, 3 \mathrm{H}), 6.10(\mathrm{~s}, 1 \mathrm{H}), 3.87$ (s, 3H), $2.25(\mathrm{~s}, 3 \mathrm{H}) .{ }^{13} \mathrm{C}$ NMR (101 MHz, Chloroform- $d$ ) $\delta$ 187.0, 168.9, 152.9, 137.6, 136.8, 134.7, $129.2,128.0 \times 2,127.2 \times 2,126.5,123.6,122.8,122.6,117.8,109.8,107.0,33.6,25.1$. HRMS (ESI): $\mathrm{m} / \mathrm{z}$ calculated for $\mathrm{C}_{20} \mathrm{H}_{19} \mathrm{~N}_{2} \mathrm{O}_{2}[\mathrm{M}+\mathrm{H}]^{+}: 319.1447$, found: 319.1442 .

\section{(Z)-N-(3-Oxo-1-phenylnon-1-en-1-yl)acetamide (3w)}

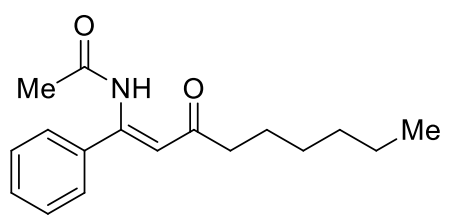

The title compound was prepared according to the typical procedure and isolated as a yellow oil (29.0 mg, $0.106 \mathrm{mmol}, 53 \%) .{ }^{1} \mathrm{H}$ NMR 
(400 MHz, Chloroform- $d$ ) $\delta 11.83$ (brs, 1H), $7.43-7.32$ (m, 5H), 5.60 (s, 1H), 2.48 (t, $J=7.5 \mathrm{~Hz}$, 2H), $2.16(\mathrm{~s}, 3 \mathrm{H}), 1.63(\mathrm{t}, J=7.1 \mathrm{~Hz}, 2 \mathrm{H}), 1.36-1.25(\mathrm{~m}, 6 \mathrm{H}), 0.92-0.85(\mathrm{~m}, 3 \mathrm{H}) .{ }^{13} \mathrm{C}$ NMR $(101$ MHz, Chloroform- $d$ ) $\delta$ 203.0, 168.9, 154.0, 135.8, 129.6, $128.0 \times 2,127.2 \times 2,108.1,43.9,31.6,28.9$, 25.0, 24.6, 22.5, 14.0. HRMS (ESI): $\mathrm{m} / \mathrm{z}$ calculated for $\mathrm{C}_{17} \mathrm{H}_{24} \mathrm{NO}_{2}[\mathrm{M}+\mathrm{H}]^{+}$: 274.1807, found: 274.1800.

(Z)- $N$-(3-Oxo-1-phenyloct-1-en-1-yl)acetamide (3x)

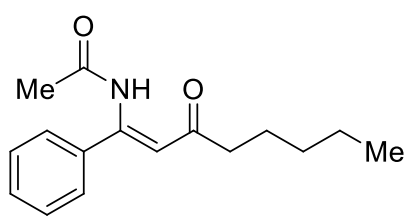

The title compound was prepared according to the typical procedure and isolated as a yellow oil (25.9 mg, $0.100 \mathrm{mmol}, 50 \%) .{ }^{1} \mathrm{H}$ NMR (400 MHz, Chloroform- $d$ ) $\delta 11.84$ (brs, $1 \mathrm{H}), 7.46-7.34$ (m, 5H), 5.61 (s, 1H), 2.49 $(\mathrm{t}, J=7.5 \mathrm{~Hz}, 2 \mathrm{H}), 2.18(\mathrm{~s}, 3 \mathrm{H}), 1.66(\mathrm{q}, J=7.4 \mathrm{~Hz}, 2 \mathrm{H}), 1.36-1.30(\mathrm{~m}, 4 \mathrm{H}), 0.91(\mathrm{t}, J=6.9 \mathrm{~Hz}, 3 \mathrm{H})$. ${ }^{13} \mathrm{C}$ NMR (101 MHz, Chloroform-d) $\delta$ 203.0, 168.9, 154.0, 135.8, 129.6, $128.0 \times 2,127.3 \times 2,108.1$, 43.9, 31.4, 25.0, 24.4, 22.5, 13.9. HRMS (ESI): $\mathrm{m} / \mathrm{z}$ calculated for $\mathrm{C}_{16} \mathrm{H}_{22} \mathrm{NO}_{2}[\mathrm{M}+\mathrm{H}]^{+}: 260.1651$, found: 260.1655 .

(Z)- $N$-(3-Oxo-1,5-diphenylpent-1-en-1-yl)acetamide (3y)<smiles>CC(=O)N/C(=C\C(=O)CCc1ccccc1)c1ccccc1</smiles>

The title compound was prepared according to the typical procedure and isolated as a yellow oil (32.3 mg, $0.110 \mathrm{mmol}, 55 \%) .{ }^{1} \mathrm{H}$ NMR $(400 \mathrm{MHz}$, Chloroform- $d$ ) $\delta 11.81$ (brs, $1 \mathrm{H}), 7.42-7.39(\mathrm{~m}, 1 \mathrm{H}), 7.38-7.34(\mathrm{~m}, 4 \mathrm{H})$, $7.34-7.29(\mathrm{~m}, 2 \mathrm{H}), 7.25-7.21(\mathrm{~m}, 3 \mathrm{H}), 5.60(\mathrm{~s}, 1 \mathrm{H}), 3.03-2.95(\mathrm{~m}, 2 \mathrm{H}), 2.89-2.81(\mathrm{~m}, 2 \mathrm{H}), 2.19$ (s, 3H). ${ }^{13} \mathrm{C}$ NMR (101 MHz, Chloroform- $d$ ) $\delta$ 201.4, 168.8, 154.3, 140.8, 135.6, 129.7, $128.5 \times 2$, $128.2 \times 2,128.0 \times 2,127.3 \times 2,126.2,107.9,45.1,30.4,25.0 . H R M S(E S I): \mathrm{m} / \mathrm{z}$ calculated for $\mathrm{C}_{19} \mathrm{H}_{21} \mathrm{NO}_{2}[\mathrm{M}+\mathrm{H}]^{+}: 294.1494$, found: 294.1485.

(Z)-N-(3-Cyclohexyl-3-oxo-1-phenylprop-1-en-1-yl)acetamide (3z)<smiles>CC(=O)N/C(=C\C(=O)C1CCCCC1)c1ccccc1</smiles>

The title compound was prepared according to the typical procedure and isolated as a yellow oil $(21.7 \mathrm{mg}, 0.080 \mathrm{mmol}, 40 \%) .{ }^{1} \mathrm{H}$ NMR (400 MHz, Chloroform- $d$ ) $\delta 11.89$ (brs, 1H), $7.45-7.34$ (m, 5H), 5.64 (s, 1H), $2.43-2.35$ (m, 1H), $2.18(\mathrm{~s}, 3 \mathrm{H}), 1.94-1.78(\mathrm{~m}, 4 \mathrm{H}), 1.75-1.64(\mathrm{~m}, 1 \mathrm{H}), 1.45-1.22(\mathrm{~m}, 5 \mathrm{H}) .{ }^{13} \mathrm{C}$ NMR $(101$ MHz, Chloroform- $d$ ) $\delta$ 205.9, 168.9, 154.6, 135.9, 129.6, $128.0 \times 2,127.3 \times 2,107.3,51.5,28.9 \times 2$, 
25.8, $25.7 \times 2$ 2, 25.0. HRMS $(E S I): \mathrm{m} / \mathrm{z}$ calculated for $\mathrm{C}_{17} \mathrm{H}_{22} \mathrm{NO}_{2}[\mathrm{M}+\mathrm{H}]^{+}: 272.1651$, found: 272.1653 .

\section{(Z)- $N$-(5,9-Dimethyl-3-oxo-1-phenyldeca-1,8-dien-1-yl)acetamide (3aa)}

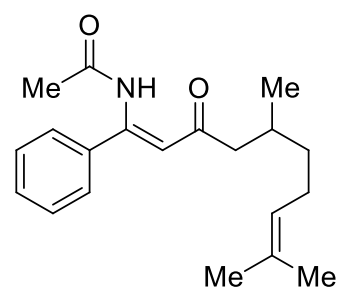

The title compound was prepared according to a modified procedure $\left(70{ }^{\circ} \mathrm{C}\right.$, with $25 \mathrm{~mol} \%$ catalyst) and isolated as a colorless oil $(27.6 \mathrm{mg}, 0.088 \mathrm{mmol}$, 44\%). ${ }^{1} \mathrm{H}$ NMR (401 MHz, Chloroform- $d$ ) $\delta 11.88$ (brs, $1 \mathrm{H}$ ), $7.43-7.35$ (m, $5 \mathrm{H}), 5.59(\mathrm{~s}, 1 \mathrm{H}), 5.10(\mathrm{tt}, J=7.1,1.3 \mathrm{~Hz}, 1 \mathrm{H}), 2.50(\mathrm{dd}, J=14.7,5.7 \mathrm{~Hz}$, 1H), $2.28(\mathrm{dd}, J=14.8,8.3 \mathrm{~Hz}, 1 \mathrm{H}), 2.18(\mathrm{~s}, 3 \mathrm{H}), 2.07-1.96(\mathrm{~m}, 3 \mathrm{H}), 1.68(\mathrm{~s}, 3 \mathrm{H}), 1.61(\mathrm{~s}, 3 \mathrm{H}), 1.41$ $-1.33(\mathrm{~m}, 1 \mathrm{H}), 1.30-1.21(\mathrm{~m}, 1 \mathrm{H}), 0.96(\mathrm{~d}, J=6.6 \mathrm{~Hz}, 3 \mathrm{H}) .{ }^{13} \mathrm{C}$ NMR $(101 \mathrm{MHz}$, Chloroform- $d) \delta$ $202.8,168.9,154.0,135.8,131.6,129.7,128.0 \times 2,127.3 \times 2,124.2,108.5,51.4,37.0,30.0,25.7$, 25.5, 25.0, 19.8, 17.7. HRMS (ESI): $\mathrm{m} / \mathrm{z}$ calculated for $\mathrm{C}_{20} \mathrm{H}_{28} \mathrm{NO}_{2}[\mathrm{M}+\mathrm{H}]^{+}$: 314.2120, found: 314.2128 .

$(1 R, 2 S, 5 R)-2-$ Isopropyl-5-methylcyclohexyl-4-((Z)-3-acetamido-3-phenylacryloyl)benzoate (3ab)<smiles>CC(=O)N/C(=C\C(=O)c1ccc(C(=O)OC2CC([N])CCC2C(C)C)cc1)c1ccccc1</smiles>
The title compound was prepared according to the typical procedure and isolated as a yellow oil $(52.6 \mathrm{mg}, 0.118 \mathrm{mmol}$, 59\%). ${ }^{1} \mathrm{H}$ NMR (401 MHz, Chloroform- $d$ ) $\delta 12.25$ (brs, $1 \mathrm{H}$ ), 8.14 $(\mathrm{d}, J=8.4 \mathrm{~Hz}, 2 \mathrm{H}), 8.01(\mathrm{~d}, J=8.5 \mathrm{~Hz}, 2 \mathrm{H}), 7.50-7.39(\mathrm{~m}, 5 \mathrm{H})$, $6.31(\mathrm{~s}, 1 \mathrm{H}), 4.96(\mathrm{td}, J=10.9,4.4 \mathrm{~Hz}, 1 \mathrm{H}), 2.26(\mathrm{~s}, 3 \mathrm{H}), 2.16-$ $2.11(\mathrm{~m}, 1 \mathrm{H}), 2.01-1.91(\mathrm{~m}, 1 \mathrm{H}), 1.80-1.70(\mathrm{~m}, 2 \mathrm{H}), 1.67-1.64(\mathrm{~m}, 1 \mathrm{H}), 1.62-1.54(\mathrm{~m}, 2 \mathrm{H})$, $1.22-1.07(\mathrm{~m}, 2 \mathrm{H}), 0.94(\mathrm{dd}, J=6.8,4.7 \mathrm{~Hz}, 6 \mathrm{H}), 0.81(\mathrm{~d}, J=6.9 \mathrm{~Hz}, 3 \mathrm{H}) .{ }^{13} \mathrm{C}$ NMR $(101 \mathrm{MHz}$, Chloroform- $d$ ) $\delta 190.9,168.8,165.2,157.2,141.9,136.0,134.2,130.0,129.8 \times 2,128.1 \times 2,127.7 \times$ $2,127.4 \times 2,104.6,75.4,47.2,40.9,34.2,31.4,26.5,25.1,23.6,22.0,20.7,16.5$. HRMS (ESI): m/z calculated for $\mathrm{C}_{28} \mathrm{H}_{34} \mathrm{NO}_{4}[\mathrm{M}+\mathrm{H}]^{+}: 448.2488$, found: 448.2483 .

(3aS,5S,6R,6aS)-5-((S)-2,2-Dimethyl-1,3-dioxolan-4-yl)-2,2-dimethyltetrahydrofuro[2,3-d][1,3]di oxol-6-yl 4-((Z)-3-acetamido-3-phenylacryloyl)benzoate (3ac) 
<smiles>CC(=O)N/C(=C\C(=O)c1ccc(C(=O)O[C@H]2[C@H]([C@H]3COC(C)(C)O3)O[C@@H]3OC(C)(C)O[C@H]23)cc1)c1ccccc1</smiles>

The title compound was prepared according to the typical procedure and isolated as a yellow oil $(60.5 \mathrm{mg}, 0.110 \mathrm{mmol}$, 55\%). ${ }^{1} \mathrm{H}$ NMR (401 MHz, Chloroform- $d$ ) $\delta 12.23$ (brs, 1H), 8.18 $-7.98(\mathrm{~m}, 4 \mathrm{H}), 7.44(\mathrm{dt}, J=13.6,7.5 \mathrm{~Hz}, 5 \mathrm{H}), 6.30(\mathrm{~s}, 1 \mathrm{H}), 5.97$ $(\mathrm{d}, J=3.8 \mathrm{~Hz}, 1 \mathrm{H}), 5.52(\mathrm{~d}, J=2.8 \mathrm{~Hz}, 1 \mathrm{H}), 4.65(\mathrm{~d}, J=3.7 \mathrm{~Hz}$, 1H), $4.43-4.28(\mathrm{~m}, 2 \mathrm{H}), 4.15-4.07(\mathrm{~m}, 2 \mathrm{H}), 2.26(\mathrm{~s}, 3 \mathrm{H}), 1.56(\mathrm{~s}, 3 \mathrm{H}), 1.42(\mathrm{~s}, 3 \mathrm{H}), 1.33(\mathrm{~s}, 3 \mathrm{H})$, 1.27 (s, 3H). ${ }^{13} \mathrm{C}$ NMR (101 MHz, Chloroform-d) $\delta 190.6,168.8,164.4,157.5,142.5,135.9,132.8$, $130.1,130.0 \times 2,128.2 \times 2,127.8 \times 2,127.4 \times 2,112.4,109.5,105.1,104.4,83.3,79.9,77.0,72.5$, 67.3, 26.8, 26.7, 26.2, 25.2, 25.1. HRMS (ESI): $\mathrm{m} / \mathrm{z}$ calculated for $\mathrm{C}_{30} \mathrm{H}_{34} \mathrm{NO}_{9}[\mathrm{M}+\mathrm{H}]^{+}:$552.2234, found: 552.2241 .

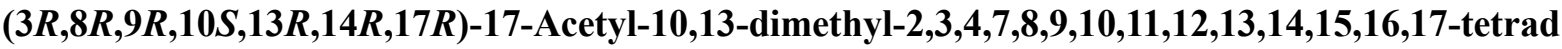
ecahydro-1H-cyclopenta[a]phenanthren-3-yl-4-((Z)-3-acetamido-3-phenylacryloyl)benzoate (3ad)

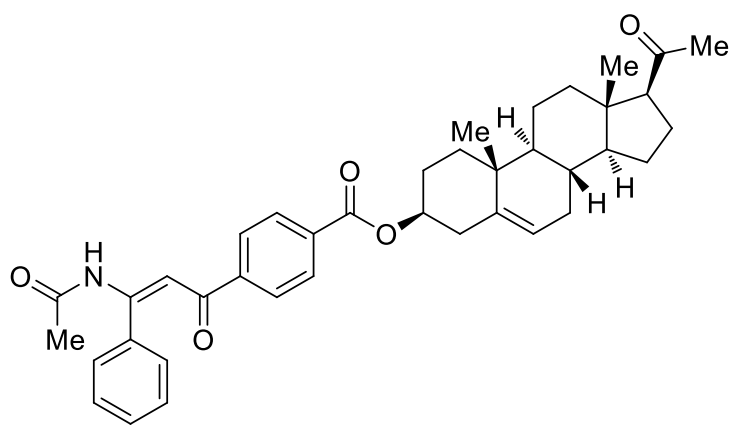

The title compound was prepared according to the typical procedure and isolated as a white solid (59.3 $\mathrm{mg}, 0.098 \mathrm{mmol}, 49 \%$ ). mp: $182.4-185.7{ }^{\circ} \mathrm{C} .{ }^{1} \mathrm{H}$ NMR (401 MHz, Chloroform-d) $\delta 12.25$ (brs, 1H), $8.13(\mathrm{~d}, J=8.5 \mathrm{~Hz}, 2 \mathrm{H}), 8.00(\mathrm{~d}, J=8.5 \mathrm{~Hz}, 2 \mathrm{H})$, $7.50-7.39(\mathrm{~m}, 5 \mathrm{H}), 6.31(\mathrm{~s}, 1 \mathrm{H}), 5.43(\mathrm{~d}, J=3.7$ $\mathrm{Hz}, 1 \mathrm{H}), 4.93-4.85(\mathrm{~m}, 1 \mathrm{H}), 2.55(\mathrm{t}, J=9.0 \mathrm{~Hz}, 1 \mathrm{H}), 2.49(\mathrm{~d}, J=7.7 \mathrm{~Hz}, 2 \mathrm{H}), 2.26(\mathrm{~s}, 3 \mathrm{H}), 2.14(\mathrm{~s}$, 3H), $2.09-2.00(\mathrm{~m}, 3 \mathrm{H}), 1.98-1.92(\mathrm{~m}, 1 \mathrm{H}), 1.80-1.64(\mathrm{~m}, 4 \mathrm{H}), 1.58-1.45(\mathrm{~m}, 4 \mathrm{H}), 1.33-1.14$ $(\mathrm{m}, 4 \mathrm{H}), 1.08(\mathrm{~s}, 3 \mathrm{H}), 0.65$ (s, 3H). ${ }^{13} \mathrm{C}$ NMR (101 MHz, Chloroform-d) $\delta 209.6,190.9,168.9,165.1$, $157.2,142.0,139.5,136.0,134.1,130.0,129.8 \times 2,128.2 \times 2,127.7 \times 2,127.4 \times 2,122.7,104.6,75.0$, 63.7, 56.8, 49.9, 44.0, 38.8, 38.1, 37.0, 36.7, 31.8, 31.8, 31.6, 27.8, 25.1, 24.5, 22.8, 21.0, 19.4, 13.2. HRMS (ESI): $\mathrm{m} / \mathrm{z}$ calculated for $\mathrm{C}_{39} \mathrm{H}_{46} \mathrm{NO}_{5}[\mathrm{M}+\mathrm{H}]^{+}: 608.3376$, found: 608.3370 .

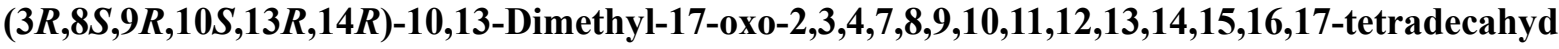
ro-1H-cyclopenta $[a]$ phenanthren-3-yl-4-((Z)-3-acetamido-3-phenylacryloyl)benzoate (3ae) 


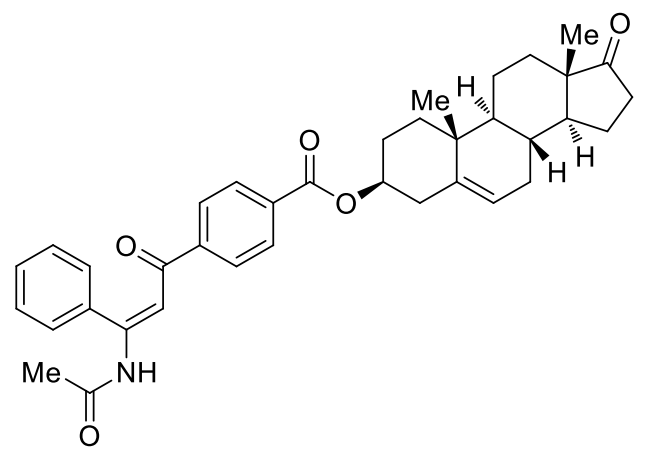

The title compound was prepared according to the typical procedure and isolated as a white solid $(59.3 \mathrm{mg}, 0.102$ mmol, 51\%). mp: $231.4-233.6{ }^{\circ} \mathrm{C} .{ }^{1} \mathrm{H}$ NMR (401 MHz, Chloroform- $d$ ) $\delta 12.25$ (brs, $1 \mathrm{H}), 8.13(\mathrm{~d}, J=8.5 \mathrm{~Hz}, 2 \mathrm{H})$, $8.00(\mathrm{~d}, J=8.5 \mathrm{~Hz}, 2 \mathrm{H}), 7.50-7.41(\mathrm{~m}, 5 \mathrm{H}), 6.31(\mathrm{~s}, 1 \mathrm{H})$, $5.46(\mathrm{~d}, J=5.5 \mathrm{~Hz}, 1 \mathrm{H}), 4.93-4.85(\mathrm{~m}, 1 \mathrm{H}), 2.51(\mathrm{~d}, J=$ $5.5 \mathrm{~Hz}, 2 \mathrm{H}), 2.49-2.42(\mathrm{~m}, 1 \mathrm{H}), 2.25(\mathrm{~s}, 3 \mathrm{H}), 2.17-1.92(\mathrm{~m}, 6 \mathrm{H}), 1.89-1.84(\mathrm{~m}, 1 \mathrm{H}), 1.74-1.66$ (m, 4H), $1.59-1.48(\mathrm{~m}, 2 \mathrm{H}), 1.34-1.25(\mathrm{~m}, 3 \mathrm{H}), 1.11(\mathrm{~s}, 3 \mathrm{H}), 0.90(\mathrm{~s}, 3 \mathrm{H}) .{ }^{13} \mathrm{C}$ NMR $(101 \mathrm{MHz}$, Chloroform- $d$ ) $\delta 221.0,190.8,168.8,165.1,157.2,141.9,139.7,135.9,134.1,130.0,129.8,128.1 \times 2$, $127.6,127.4 \times 2,126.0,122.2,104.5,74.9,51.7,50.1,47.5,38.1,36.9,36.8,35.8,31.4,31.4,30.8$, 27.7, 25.1, 21.9, 20.3, 19.4, 13.5, 1.0. HRMS (ESI): $\mathrm{m} / \mathrm{z}$ calculated for $\mathrm{C}_{37} \mathrm{H}_{42} \mathrm{NO}_{5}[\mathrm{M}+\mathrm{H}]^{+}$: 580.3063, found: 580.3071 .

(Z)- $N$-(1-(4-Fluorophenyl)-3-oxo-3-phenylprop-1-en-1-yl)acetamide (4a)<smiles>CC(=O)N/C(=C\C(=O)c1ccccc1)c1ccc(F)cc1</smiles>

The title compound was prepared according to the typical procedure and isolated as a yellow oil $(44.7 \mathrm{mg}, 0.16 \mathrm{mmol}, 79 \%) .{ }^{1} \mathrm{H}$ NMR $(400 \mathrm{MHz}$, Chloroform- $d$ ) $\delta 12.28$ (brs, 1H), $8.00-7.93(\mathrm{~m}, 2 \mathrm{H}), 7.61-7.54(\mathrm{~m}, 1 \mathrm{H}), 7.53$ - $7.42(\mathrm{~m}, 4 \mathrm{H}), 7.10(\mathrm{t}, J=8.7 \mathrm{~Hz}, 2 \mathrm{H}), 6.30(\mathrm{~s}, 1 \mathrm{H}), 2.26(\mathrm{~s}, 3 \mathrm{H}) .{ }^{13} \mathrm{C}$ NMR $(101 \mathrm{MHz}$, Chloroform- $d) \delta 191.6,169.0,163.6\left(\mathrm{~d}, J_{\mathrm{C}-\mathrm{F}}=250.2 \mathrm{~Hz}\right), 155.1,138.5,132.8,132.1\left(\mathrm{~d}, J_{\mathrm{C}-\mathrm{F}}=3.7 \mathrm{~Hz}\right)$, $129.4\left(\mathrm{~d}, J_{\mathrm{C}-\mathrm{F}}=8.7 \mathrm{~Hz}\right), 128.7 \times 2,127.8 \times 2,115.2\left(\mathrm{~d}, J_{\mathrm{C}-\mathrm{F}}=22.0 \mathrm{~Hz}\right), 104.7,25.1 .{ }^{19} \mathrm{~F}$ NMR $(376$ $\mathrm{MHz}$, Chloroform- $d$ ) $\delta$-110.41. HRMS (ESI): $\mathrm{m} / \mathrm{z}$ calculated for $\mathrm{C}_{17} \mathrm{H}_{15} \mathrm{FNO}_{2}[\mathrm{M}+\mathrm{H}]^{+}: 284.1087$, found: 284.1081 .

\section{(Z)-N-(1-(4-Chlorophenyl)-3-oxo-3-phenylprop-1-en-1-yl)acetamide (4b)}<smiles>CC(=O)N/C(=C\C(=O)c1ccccc1)c1ccc(Cl)cc1</smiles>

The title compound was prepared according to the typical procedure and isolated as a yellow oil (42.2 $\mathrm{mg}, 0.14 \mathrm{mmol}, 71 \%) .{ }^{1} \mathrm{H}$ NMR (400 MHz, Chloroform- $d$ ) $\delta 12.25$ (brs, 1H), $7.99-7.92(\mathrm{~m}, 2 \mathrm{H}), 7.61-7.55(\mathrm{~m}, 1 \mathrm{H})$, 7.49 (ddd, $J=8.3,6.6,1.3 \mathrm{~Hz}, 2 \mathrm{H}), 7.44-7.34(\mathrm{~m}, 4 \mathrm{H}), 6.30(\mathrm{~s}, 1 \mathrm{H}), 2.25$ (s, 3H). ${ }^{13} \mathrm{C}$ NMR $(101$ MHz, Chloroform- $d$ ) $\delta 191.6,168.9,154.8,138.4,135.8,134.6,132.9,128.7 \times 4,128.3 \times 2,127.8 \times$ 2, 104.8, 25.0. HRMS (ESI): $\mathrm{m} / \mathrm{z}$ calculated for $\mathrm{C}_{17} \mathrm{H}_{15} \mathrm{ClNO}_{2}[\mathrm{M}+\mathrm{H}]^{+}: 300.0791$, found: 300.0785 . 
(Z)-N-(1-(4-Bromophenyl)-3-oxo-3-phenylprop-1-en-1-yl)acetamide (4c)<smiles>CC(=O)N/C(=C\C(=O)c1ccccc1)c1ccc(Br)cc1</smiles>

The title compound was prepared according to the typical procedure and isolated as a white solid (42.3 mg, $0.124 \mathrm{mmol}, 62 \%)$. mp: $106.8-107.9^{\circ} \mathrm{C}$.

${ }^{1} \mathrm{H}$ NMR (400 MHz, Chloroform- $d$ ) $\delta 12.24$ (brs, 1H), $8.01-7.89$ (m, 2H), $7.62-7.45(\mathrm{~m}, 5 \mathrm{H}), 7.37-7.30(\mathrm{~m}, 2 \mathrm{H}), 6.30(\mathrm{~s}, 1 \mathrm{H}), 2.25(\mathrm{~s}, 3 \mathrm{H}) .{ }^{13} \mathrm{C}$ NMR $(101 \mathrm{MHz}$, Chloroform-d) $\delta 191.6,168.9,154.9,138.3,135.1,132.9,131.3 \times 2,128.9 \times 2,128.7 \times 2,127.8 \times 2$, 124.1, 104.8, 25.0. HRMS (ESI): $\mathrm{m} / \mathrm{z}$ calculated for $\mathrm{C}_{17} \mathrm{H}_{15} \mathrm{BrNO}_{2}[\mathrm{M}+\mathrm{H}]^{+}$: 344.0286, found: 344.0286.

\section{(Z)-N-(3-Oxo-3-phenyl-1-(p-tolyl)prop-1-en-1-yl)acetamide (4d)}<smiles>CC(=O)N/C(=C\C(=O)c1ccccc1)c1ccc(C)cc1</smiles>

The title compound was prepared according to the typical procedure and isolated as a yellow oil (35.5 mg, $0.128 \mathrm{mmol}, 64 \%)$. ${ }^{1} \mathrm{H}$ NMR (400 MHz, Chloroform- $d$ ) $\delta 12.26$ (brs, 1H), $8.03-7.90(\mathrm{~m}, 2 \mathrm{H}), 7.60-7.54(\mathrm{~m}, 1 \mathrm{H})$, $7.52-7.46(\mathrm{~m}, 2 \mathrm{H}), 7.41-7.36(\mathrm{~m}, 2 \mathrm{H}), 7.26-7.20(\mathrm{~m}, 2 \mathrm{H}), 6.33(\mathrm{~s}, 1 \mathrm{H}), 2.41(\mathrm{~s}, 3 \mathrm{H}), 2.25(\mathrm{~s}, 3 \mathrm{H})$.

${ }^{13} \mathrm{C}$ NMR (101 MHz, Chloroform- $d$ ) $\delta$ 191.6, 169.0, 156.4, 140.2, 138.7, 133.2, 132.6, $128.8 \times 2$, $128.6 \times 2,127.8 \times 2,127.4 \times 2,104.4,25.2,21.4 . \mathrm{HRMS}(\mathrm{ESI}): \mathrm{m} / \mathrm{z}$ calculated for $\mathrm{C}_{18} \mathrm{H}_{18} \mathrm{NO}_{2}[\mathrm{M}+$ $\mathrm{H}]^{+}: 280.1338$, found: 280.1333 .

\section{(Z)-N-(1-(4-Cyanophenyl)-3-oxo-3-phenylprop-1-en-1-yl)acetamide (4e)}<smiles>CC(=O)N/C(=C\C(=O)c1ccccc1)c1ccc(C#N)cc1</smiles>

The title compound was prepared according to the typical procedure and isolated as a white solid $(46.5 \mathrm{mg}, 0.160 \mathrm{mmol}, 80 \%)$. mp: $157.9-159.2{ }^{\circ} \mathrm{C}$. ${ }^{1} \mathrm{H}$ NMR (400 MHz, Chloroform- $d$ ) $\delta 12.24$ (brs, 1H), $8.02-7.91$ (m, 2H), $7.73-7.67(\mathrm{~m}, 2 \mathrm{H}), 7.63-7.57(\mathrm{~m}, 1 \mathrm{H}), 7.57-7.53(\mathrm{~m}, 2 \mathrm{H}), 7.50(\mathrm{t}, J=7.6 \mathrm{~Hz}, 2 \mathrm{H}), 6.30(\mathrm{~s}, 1 \mathrm{H})$, 2.26 (s, 3H). ${ }^{13} \mathrm{C}$ NMR (101 MHz, Chloroform- $d$ ) $\delta$ 191.7, 168.9, 153.7, 140.9, 138.1, 133.2, $131.9 \times$ $2,128.8 \times 2,128.0 \times 2,127.9 \times 2,118.4,113.1,105.5,24.9$. HRMS $(E S I): \mathrm{m} / \mathrm{z}$ calculated for $\mathrm{C}_{18} \mathrm{H}_{15} \mathrm{~N}_{2} \mathrm{O}_{2}[\mathrm{M}+\mathrm{H}]^{+}:$291.1134, found: 291.1129 . 
<smiles>CC(=O)N/C(=C\C(=O)c1ccccc1)c1ccc(C(F)(F)F)cc1</smiles>

The title compound was prepared according to the typical procedure and isolated as a white solid (46.2 mg, $0.138 \mathrm{mmol}, 69 \%)$. mp: $120.4-121.6{ }^{\circ} \mathrm{C}$.

${ }^{1} \mathrm{H}$ NMR (400 MHz, Chloroform- $d$ ) $\delta 12.28$ (brs, 1H), $8.00-7.94$ (m, 2H), $7.67(\mathrm{~d}, J=8.1 \mathrm{~Hz}, 2 \mathrm{H}), 7.62-7.54(\mathrm{~m}, 3 \mathrm{H}), 7.53-7.47(\mathrm{~m}, 2 \mathrm{H}), 6.31(\mathrm{~s}, 1 \mathrm{H}), 2.26(\mathrm{~s}, 3 \mathrm{H}) .{ }^{13} \mathrm{C}$ NMR (101 MHz, Chloroform- $d) \delta$ 191.7, 168.9, 154.4, 139.9 (d, $\left.J_{\mathrm{C}-\mathrm{F}}=1.5 \mathrm{~Hz}\right), 138.2,133.1,131.3(\mathrm{~d}$, $\left.J_{\mathrm{C}-\mathrm{F}}=32.7 \mathrm{~Hz}\right), 128.7 \times 2,127.8 \times 2,127.6 \times 2,125.1\left(\mathrm{q}, J_{\mathrm{C}-\mathrm{F}}=4.1 \mathrm{~Hz}\right), 123.8\left(\mathrm{~d}, J_{\mathrm{C}-\mathrm{F}}=272.5 \mathrm{~Hz}\right)$, 105.3, 24.9. ${ }^{19} \mathrm{~F}$ NMR (376 MHz, Chloroform- $d$ ) $\delta$-62.65. HRMS (ESI): $\mathrm{m} / \mathrm{z}$ calculated for $\mathrm{C}_{18} \mathrm{H}_{15} \mathrm{~F}_{3} \mathrm{NO}_{2}[\mathrm{M}+\mathrm{H}]^{+}: 334.1055$, found: 334.1046 .

\section{(Z)-N-(1-(3-Methoxyphenyl)-3-oxo-3-phenylprop-1-en-1-yl)acetamide (4g)}<smiles>COc1cccc(/C(=C/C(=O)c2ccccc2)NC(C)=O)c1</smiles>

The title compound was prepared according to the typical procedure and isolated as a white solid (42.3 mg, $0.14 \mathrm{mmol}, 72 \%)$. mp: $99.8-101.4{ }^{\circ} \mathrm{C} .{ }^{1} \mathrm{H}$ NMR (400 MHz, Chloroform- $d$ ) $\delta 12.22$ (brs, 1H), 8.00 - 7.94 (m, 2H), 7.61 $-7.54(\mathrm{~m}, 1 \mathrm{H}), 7.48(\mathrm{t}, J=7.5 \mathrm{~Hz}, 2 \mathrm{H}), 7.33(\mathrm{t}, J=7.8 \mathrm{~Hz}, 1 \mathrm{H}), 7.07-7.05(\mathrm{~m}, 1 \mathrm{H}), 7.02-6.96(\mathrm{~m}$, 2H), $6.34(\mathrm{~s}, 1 \mathrm{H}), 3.84(\mathrm{~s}, 3 \mathrm{H}), 2.25(\mathrm{~s}, 3 \mathrm{H}) .{ }^{13} \mathrm{C}$ NMR (101 MHz, Chloroform- $d$ ) $\delta$ 191.7, 168.8, 159.2, 155.9, 138.5, 137.6, 132.8, 129.1, $128.6 \times 2,127.8 \times 2,119.8,115.0,113.2,104.7,55.3,25.1$. HRMS (ESI): $\mathrm{m} / \mathrm{z}$ calculated for $\mathrm{C}_{18} \mathrm{H}_{18} \mathrm{NO}_{3}[\mathrm{M}+\mathrm{H}]^{+}: 296.1287$, found: 296.1281 .

\section{(Z)- $N$-(1-(2-Fluorophenyl)-3-oxo-3-phenylprop-1-en-1-yl)acetamide (4h)}

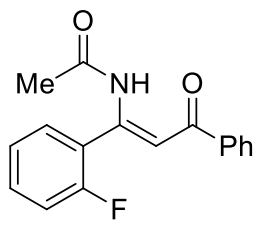

The title compound was prepared according to the typical procedure and isolated as a white solid (39.2 mg, $0.14 \mathrm{mmol}, 69 \%)$. mp: $136.8-138.3{ }^{\circ} \mathrm{C} .{ }^{1} \mathrm{H}$ NMR (400 MHz, Chloroform- $d$ ) $\delta 12.50$ (brs, 1H), 7.96 (dd, $J=8.4,1.4 \mathrm{~Hz}, 2 \mathrm{H}), 7.60-7.54$ (m, 1H), $7.51-7.45(\mathrm{~m}, 2 \mathrm{H}), 7.45-7.39(\mathrm{~m}, 1 \mathrm{H}), 7.37$ (dd, $J=7.4,1.7 \mathrm{~Hz}, 1 \mathrm{H}), 7.21(\mathrm{td}, J=7.5,1.1$ $\mathrm{Hz}, 1 \mathrm{H}), 7.11-7.06(\mathrm{~m}, 1 \mathrm{H}), 6.24$ (s, 1H), $2.24(\mathrm{~s}, 3 \mathrm{H}) .{ }^{13} \mathrm{C}$ NMR (101 MHz, Chloroform- $d$ ) $\delta 191.7$, $168.5,159.5\left(\mathrm{~d}, J_{\mathrm{C}-\mathrm{F}}=249.2 \mathrm{~Hz}\right), 150.6,138.4,132.8,131.2\left(\mathrm{~d}, J_{\mathrm{C}-\mathrm{F}}=8.3 \mathrm{~Hz}\right), 129.0\left(\mathrm{~d}, J_{\mathrm{C}-\mathrm{F}}=3.0 \mathrm{~Hz}\right)$, $128.6 \times 2,127.8 \times 2,124.6\left(\mathrm{~d}, J_{\mathrm{C}-\mathrm{F}}=14.6 \mathrm{~Hz}\right), 124.0\left(\mathrm{~d}, J_{\mathrm{C}-\mathrm{F}}=3.5 \mathrm{~Hz}\right), 115.2\left(\mathrm{~d}, J_{\mathrm{C}-\mathrm{F}}=21.2 \mathrm{~Hz}\right)$, $104.4\left(\mathrm{~d}, J_{\mathrm{C}-\mathrm{F}}=1.4 \mathrm{~Hz}\right), 24.9 .{ }^{19} \mathrm{~F}$ NMR $(376 \mathrm{MHz}$, Chloroform- $d$ ) $\delta-114.21$. HRMS (ESI): m/z calculated for $\mathrm{C}_{17} \mathrm{H}_{15} \mathrm{FNO}_{2}[\mathrm{M}+\mathrm{H}]^{+}: 284.1087$, found: 284.1082 . 


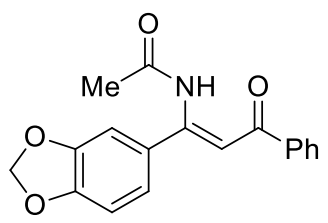

The title compound was prepared according to the typical procedure and isolated as a white solid (31.2 mg, $0.100 \mathrm{mmol}, 50 \%)$. mp: $140.8-143.9^{\circ} \mathrm{C}$.

${ }^{1} \mathrm{H}$ NMR (400 MHz, Chloroform- $d$ ) $\delta 12.18$ (brs, 1H), 7.99 - 7.92 (m, 2H), $7.60-7.53(\mathrm{~m}, 1 \mathrm{H}), 7.48(\mathrm{t}, J=7.5 \mathrm{~Hz}, 2 \mathrm{H}), 7.03(\mathrm{dd}, J=8.1,1.8 \mathrm{~Hz}, 1 \mathrm{H}), 6.94(\mathrm{~d}, J=1.7 \mathrm{~Hz}, 1 \mathrm{H})$, $6.84(\mathrm{~d}, J=8.1 \mathrm{~Hz}, 1 \mathrm{H}), 6.32(\mathrm{~s}, 1 \mathrm{H}), 6.02(\mathrm{~s}, 2 \mathrm{H}), 2.25(\mathrm{~s}, 3 \mathrm{H}) .{ }^{13} \mathrm{C}$ NMR $(101 \mathrm{MHz}$, Chloroform- $d)$ $\delta 191.5,169.1,155.7,149.3,147.5,138.6,132.7,130.0,128.6 \times 2,127.8 \times 2,121.8,108.1,108.0$, 104.3, 101.5, 25.2. HRMS (ESI): $\mathrm{m} / \mathrm{z}$ calculated for $\mathrm{C}_{18} \mathrm{H}_{16} \mathrm{NO}_{4}[\mathrm{M}+\mathrm{H}]^{+}: 310.1079$, found: 310.1076 .

\section{(Z)-N-(1-(Naphthalen-2-yl)-3-oxo-3-phenylprop-1-en-1-yl)acetamide (4j)}

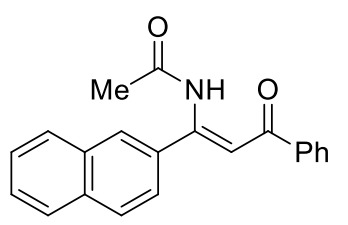

The title compound was prepared according to the typical procedure and isolated as a yellow oil (50.8 mg, $0.16 \mathrm{mmol}, 81 \%) .{ }^{1} \mathrm{H}$ NMR (400 MHz, Chloroform- $d$ ) $\delta 12.40$ (brs, 1H), $8.04-7.98(\mathrm{~m}, 3 \mathrm{H}), 7.94-7.83(\mathrm{~m}, 3 \mathrm{H})$, $7.62-7.47$ (m, 6H), 6.46 (s, 1H), 2.29 (s, 3H). ${ }^{13} \mathrm{C}$ NMR (101 MHz, Chloroform-d) $\delta$ 191.6, 168.9, $156.2,138.6,133.9,133.9,132.8,132.7,128.7 \times 2,128.5,127 . \times 2,127.7,127.4,127.0,126.5,126.4$, 125.1, 105.0, 25.1. HRMS (ESI): $\mathrm{m} / \mathrm{z}$ calculated for $\mathrm{C}_{21} \mathrm{H}_{18} \mathrm{NO}_{2}[\mathrm{M}+\mathrm{H}]^{+}: 316.1338$, found: 316.1331 .

\section{(Z)-N-(1-(Furan-2-yl)-3-oxo-3-phenylprop-1-en-1-yl)acetamide (4k)}

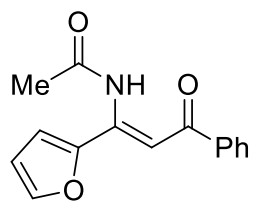

The title compound was prepared according to the typical procedure and isolated as a yellow oil (20.6 mg, $0.082 \mathrm{mmol}, 41 \%) .{ }^{1} \mathrm{H}$ NMR (400 MHz, Chloroform- $d$ ) $\delta$ 12.00 (brs, 1H), $8.01-7.95(\mathrm{~m}, 2 \mathrm{H}), 7.63-7.53(\mathrm{~m}, 2 \mathrm{H}), 7.49(\mathrm{t}, J=7.5 \mathrm{~Hz}, 2 \mathrm{H})$, $7.01(\mathrm{~d}, J=3.5 \mathrm{~Hz}, 1 \mathrm{H}), 6.78(\mathrm{~s}, 1 \mathrm{H}), 6.54(\mathrm{dd}, J=3.5,1.8 \mathrm{~Hz}, 1 \mathrm{H}), 2.29(\mathrm{~s}, 3 \mathrm{H}) .{ }^{13} \mathrm{C}$ NMR $(101$ $\mathrm{MHz}$, Chloroform- $d$ ) $\delta 191.9,169.1,147.8,144.6,143.5,138.7,132.8,128.6 \times 2,127.8 \times 2,115.9$, 112.3, 101.9, 25.1. HRMS (ESI): $\mathrm{m} / \mathrm{z}$ calculated for $\mathrm{C}_{15} \mathrm{H}_{14} \mathrm{NO}_{3}[\mathrm{M}+\mathrm{H}]^{+}: 256.0974$, found: 256.0968 .

\section{(Z)-N-(3-Oxo-3-phenyl-1-(thiophen-2-yl)prop-1-en-1-yl)acetamide (4l)}

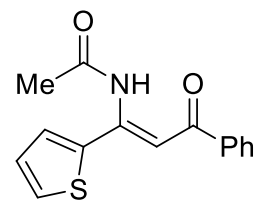

The title compound was prepared according to the typical procedure and isolated as a yellow oil (24.6 mg, $0.091 \mathrm{mmol}, 46 \%) .{ }^{1} \mathrm{H}$ NMR (400 MHz, Chloroform- $d$ ) $\delta$ 12.05 (brs, 1H), $7.99-7.92(\mathrm{~m}, 2 \mathrm{H}), 7.61-7.54(\mathrm{~m}, 1 \mathrm{H}), 7.53-7.45(\mathrm{~m}, 3 \mathrm{H})$,

$7.41(\mathrm{dd}, J=3.7,1.2 \mathrm{~Hz}, 1 \mathrm{H}), 7.09(\mathrm{dd}, J=5.1,3.7 \mathrm{~Hz}, 1 \mathrm{H}), 6.55(\mathrm{~s}, 1 \mathrm{H}), 2.28(\mathrm{~s}, 3 \mathrm{H}) .{ }^{13} \mathrm{C} \mathrm{NMR}$ $(101 \mathrm{MHz}$, Chloroform- $d$ ) $\delta 191.5,169.2,148.7,138.5,137.6,132.8,129.5,128.7 \times 2,128.5,127.8 \times$ 
2, 127.4, 104.7, 25.2. HRMS (ESI): $\mathrm{m} / \mathrm{z}$ calculated for $\mathrm{C}_{15} \mathrm{H}_{14} \mathrm{NO}_{2} \mathrm{~S}[\mathrm{M}+\mathrm{H}]^{+}:$272.0745, found: 272.0739.

\section{$N$-(2-Benzoyl-1 $H$-inden-3-yl)acetamide (4m)}

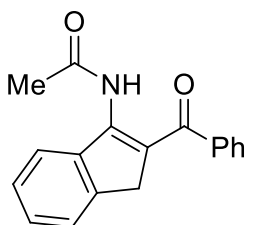

The title compound was prepared according to the typical procedure and isolated as a white solid (22.6 mg, $0.081 \mathrm{mmol}, 41 \%)$. mp: 133.0 - $134.0{ }^{\circ} \mathrm{C} .{ }^{1} \mathrm{H}$ NMR (400 MHz, Chloroform- $d$ ) $\delta 11.72$ (brs, 1H), 8.31 (d, $J=7.3 \mathrm{~Hz}, 1 \mathrm{H}), 7.76-7.69$ (m, 2H), $7.58-7.35(\mathrm{~m}, 6 \mathrm{H}), 3.80(\mathrm{~s}, 2 \mathrm{H}), 2.36(\mathrm{~s}, 3 \mathrm{H}) .{ }^{13} \mathrm{C}$ NMR (101 MHz, Chloroform-d) $\delta$ 194.6, $169.2,153.6,144.8,140.7,137.5,131.3,129.6,128.4 \times 2,127.7,127.6 \times 2,126.9,123.9,119.6,37.9$, 24.9. HRMS (ESI): $\mathrm{m} / \mathrm{z}$ calculated for $\mathrm{C}_{18} \mathrm{H}_{16} \mathrm{NO}_{2}[\mathrm{M}+\mathrm{H}]^{+}: 278.1181$, found: 278.1176 .

\section{(Z)-N-(3-Oxo-1,3-diphenylprop-1-en-1-yl)propionamide (4n)}

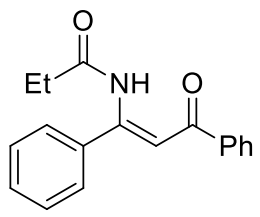

The title compound was prepared according to the typical procedure and isolated as a white solid (42.5 mg, $0.152 \mathrm{mmol}, 76 \%)$. mp: $84.8-86.2{ }^{\circ} \mathrm{C} .{ }^{1} \mathrm{H}$ NMR (400 MHz, Chloroform- $d$ ) $\delta 12.33$ (brs, 1H), $8.01-7.94(\mathrm{~m}, 2 \mathrm{H}), 7.60-7.54(\mathrm{~m}, 1 \mathrm{H})$, $7.52-7.39(\mathrm{~m}, 7 \mathrm{H}), 6.32(\mathrm{~s}, 1 \mathrm{H}), 2.54(\mathrm{q}, J=7.5 \mathrm{~Hz}, 2 \mathrm{H}), 1.23(\mathrm{t}, J=7.5 \mathrm{~Hz}, 3 \mathrm{H}) .{ }^{13} \mathrm{C}$ NMR $(101$ MHz, Chloroform-d) $\delta 191.7,172.7,156.5,138.6,136.4,132.7,129.7,128.6 \times 2,128.0 \times 2,127.8 \times$ 2, $127.3 \times 2,104.6,31.2,9.0$. HRMS (ESI): $\mathrm{m} / \mathrm{z}$ calculated for $\mathrm{C}_{18} \mathrm{H}_{18} \mathrm{NO}_{2}[\mathrm{M}+\mathrm{H}]^{+}: 280.1338$, found: 280.1333.

\section{(Z)- $N$-(3-Oxo-1,3-diphenylprop-1-en-1-yl)isobutyramide (4o)}

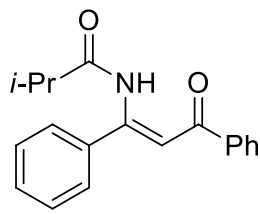

The title compound was prepared according to a modified procedure $(18 \mathrm{~h})$ and isolated as a yellow oil (40.5 mg, $0.138 \mathrm{mmol}, 69 \%, 18 \mathrm{~h}) .{ }^{1} \mathrm{H}$ NMR (400 MHz, Chloroform- $d$ ) $\delta 12.37$ (brs. 1H), $8.01-7.94(\mathrm{~m}, 2 \mathrm{H}), 7.60-7.54$ (m, 1H), $7.52-$ 7.37 (m, 7H), 6.33 (s, 1H), 2.70 (p, $J=7.0 \mathrm{~Hz}, 1 \mathrm{H}), 1.31(\mathrm{~s}, 3 \mathrm{H}), 1.29(\mathrm{~s}, 3 \mathrm{H}) .{ }^{13} \mathrm{C}$ NMR $(101 \mathrm{MHz}$ Chloroform- $d$ ) $\delta 191.8,176.0,156.8,138.7,136.4,132.7,129.7,128.7 \times 2,128.1 \times 2,127.8 \times 2$, 127.2 $\times 2,104.8,37.0,19.3$. HRMS (ESI): $\mathrm{m} / \mathrm{z}$ calculated for $\mathrm{C}_{19} \mathrm{H}_{20} \mathrm{NO}_{2}[\mathrm{M}+\mathrm{H}]^{+}: 294.1494$, found: 294.1490. 


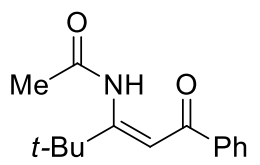

The title compound was prepared according to a modified procedure $(5 \mathrm{~h})$ and isolated as a white solid (29.8 $\mathrm{mg}, 0.122 \mathrm{mmol}, 61 \%) . \mathrm{mp}: 54.5-57.2{ }^{\circ} \mathrm{C} .{ }^{1} \mathrm{H}$ NMR (400 MHz, Chloroform- $d$ ) $\delta 12.49$ (brs. 1H), 7.93 - 7.87 (m, 2H), 7.58 - 7.51 (m, 1H), $7.51-7.43(\mathrm{~m}, 2 \mathrm{H}), 6.41(\mathrm{~s}, 1 \mathrm{H}), 2.26(\mathrm{~s}, 3 \mathrm{H}), 1.41(\mathrm{~s}, 9 \mathrm{H}) .{ }^{13} \mathrm{C}$ NMR (101 MHz, Chloroform- $d$ ) $\delta 192.5,167.9,167.1,139.1,132.5,128.6 \times 2,127.7 \times 2,101.1,36.8,28.6,25.9$. HRMS $(\mathrm{ESI}): \mathrm{m} / \mathrm{z}$ calculated for $\mathrm{C}_{15} \mathrm{H}_{20} \mathrm{NO}_{2}[\mathrm{M}+\mathrm{H}]^{+}:$246.1494, found: 246.1494 .

\section{(Z)-N-(1-(Adamantan-1-yl)-3-oxo-3-phenylprop-1-en-1-yl)acetamide (4q)}

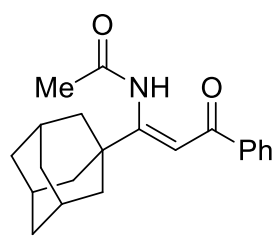

The title compound was prepared according to a modified procedure $\left(90{ }^{\circ} \mathrm{C}\right.$, with $25 \mathrm{~mol} \%$ catalyst) and isolated as a colorless oil (30.4 mg, $0.094 \mathrm{mmol}, 47 \%) .{ }^{1} \mathrm{H}$ NMR (401 MHz, Chloroform-d) $\delta 12.01$ (brs, 1H), 7.93 - 7.89 (m, 2H), 7.57 $7.51(\mathrm{~m}, 1 \mathrm{H}), 7.49-7.43(\mathrm{~m}, 2 \mathrm{H}), 6.37(\mathrm{~s}, 1 \mathrm{H}), 2.24(\mathrm{~s}, 3 \mathrm{H}), 2.08(\mathrm{~s}, 9 \mathrm{H}), 1.78(\mathrm{t}$, $J=14.5 \mathrm{~Hz}, 6 \mathrm{H}) .{ }^{13} \mathrm{C}$ NMR $(101 \mathrm{MHz}$, Chloroform- $d) \delta 192.5,168.0,166.5,139.2,132.4,128.5 \times 2$, $127.8 \times 2,102.3,39.1 \times 3,38.8,36.4 \times 3,28.4 \times 3,25.8$. HRMS $(\mathrm{ESI}): \mathrm{m} / \mathrm{z}$ calculated for $\mathrm{C}_{21} \mathrm{H}_{26} \mathrm{NO}_{2}$ $[\mathrm{M}+\mathrm{H}]^{+}:$324.1964, found: 324,1965 .

\section{$N$-((Z)-1-((8S,9S,10R,13S,14S)-3-Methoxy-10,13-dimethyl-2,3,4,7,8,9,10,11,12,13,14,15,16,17-tetr} adecahydro-1 $H$-cyclopenta[ $a]$ phenanthren-17-yl)-3-oxo-3-phenylprop-1-en-1-yl)acetamide (4r)

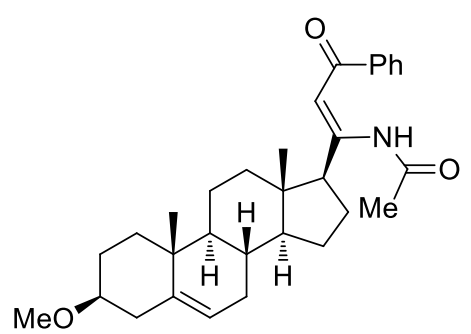

The title compound was prepared according to the typical procedure and isolated as a white solid (44.7 mg, $0.094 \mathrm{mmol}, 47 \%) . \mathrm{mp:} 149.9$ - 163.2 ${ }^{\circ} \mathrm{C} .{ }^{1} \mathrm{H}$ NMR (401 MHz, Chloroform- $d$ ) $\delta 7.92-7.87$ (m, 2H), $7.59-7.52(\mathrm{~m}, 1 \mathrm{H}), 7.47(\mathrm{dd}, J=8.3,6.7 \mathrm{~Hz}, 2 \mathrm{H}), 6.34(\mathrm{~s}, 1 \mathrm{H}), 5.41$ - $5.32(\mathrm{~m}, 1 \mathrm{H}), 4.42(\mathrm{dd}, J=10.5,8.8 \mathrm{~Hz}, 1 \mathrm{H}), 3.36(\mathrm{~s}, 3 \mathrm{H}), 3.07$ (ddd, $J=11.2,6.8,4.4 \mathrm{~Hz}, 1 \mathrm{H}), 2.39$ (ddd, $J=13.2,4.7,2.3 \mathrm{~Hz}, 1 \mathrm{H}), 2.22$ (s, 3H), $2.19-1.75$ (m, 8H), $1.73-1.23(\mathrm{~m}, 11 \mathrm{H}), 1.00(\mathrm{~s}, 3 \mathrm{H}), 0.69$ (s, 3H). ${ }^{13} \mathrm{C}$ NMR (101 MHz, Chloroform-d) $\delta$ 191.5, $170.3,162.2,140.9,139.3,132.4,128.6 \times 2,127.6 \times 2,121.3,100.8,80.2,56.6,55.6,50.0,48.9,46.0$, 38.7, 37.1, 36.9, 36.3, 32.5, 31.8, 27.9, 26.2, 25.8, 24.4, 21.0, 19.4, 13.6. HRMS (ESI): m/z calculated for $\mathrm{C}_{31} \mathrm{H}_{42} \mathrm{NO}_{3}[\mathrm{M}+\mathrm{H}]^{+}$: 476.3165, found: 476.3161 . 


\section{References}

(1) Zhao, M.-N.; Yu, L.; Hui, R.-R.; Ren, Z.-H.; Wang, Y.-Y.; Guan, Z.-H. Iron-Catalyzed Dehydrogenative [4 + 2] Cycloaddition of Tertiary Anilines and Enamides for the Synthesis of Tetrahydroquinolines with Amido-Substituted Quaternary Carbon Centers. ACS Catal. 2016, 6, 3473-3477.

(2) Li, C.; Wang, S.-M.; Qin, H.-L. A Rh-Catalyzed Air and Moisture Tolerable Aldehyde (Ketone)-Directed Fluorosulfonylvinylation of Aryl C(sp²)-H Bonds. Org. Lett. 2018, 20, 4699-4703.

(3) Zheng, Y.; Li, X.; Ren, C.; Zhang-Negrerie, D.; Du, Y.; Zhao, K. Synthesis of Oxazoles from Enamides via Phenyliodine Diacetate-Mediated Intramolecular Oxidative Cyclization. J. Org. Chem. 2012, 77, 10353-10361.

(4) Yamamoto, S.-i.; Okamoto, K.; Murakoso, M.; Kuninobu, Y.; Takai, K. Rhenium-Catalyzed Regioselective Synthesis of Multisubstituted Pyridines from $\beta$-Enamino Ketones and Alkynes via $\mathrm{C}-\mathrm{C}$ Bond Cleavage. $\operatorname{Org}$. Lett. 2012, 14, 3182-3185.

(5) Amer, A. M.; Attia, I. A. G.; El-Mobayad, M.; Asker, S. On the Chemistry of Cinnoline III: Condensation Reactions of (4-Amino-cinnolin-3-yl)-phenyl-methanone and 4-Amino-3-cinnoline-carbonitrile. Polish J. Chem. 2000, 74, 681-686.

(6) Lechel, T.; Reissig, H.-U. New 5-Alkoxypyrimidine Derivatives from $\beta$-Alkoxy $\beta$-Keto Enamides and Ammonium Salts. Eur. J. Org. Chem. 2010, 2555-2564.

(7) Sviridov, S. I.; Vasil'evb, A. A.; Shorshnev, S. V. Straightforward Transformation of Isoxazoles into Pyrazoles: Renewed and Improved. Tetrahedron 2007, 63, 12195-12201.

(8) Debbarma, S.; Bera, S. S.; Maji. M. S. Harnessing Stereospecific Z-Enamides through Silver-Free Cp*Rh(III) Catalysis by Using Isoxazoles as Masked Electrophiles. Org. Lett. 2019, 21, 835-839.

(9) Engel, D. A.; Dudley, G. B. Olefination of Ketones Using a Gold(III)-Catalyzed Meyer-Schuster Rearrangement. Org. Lett. 2006, 8, 4027-4029.

(10) For the preparation of deuterated benzaldehyde, see: Kim, S.; Ginsbach, J. W.; Lee, J. Y.; Peterson, R. L.; Liu, J. J.; Siegler, M. A.; Sarjeant, A. A.; Solomon, E. I.; Karlin, K. D. Amine Oxidative $N$-Dealkylation via Cupric Hydroperoxide Cu-OOH Homolytic Cleavage Followed by Site-Specific Fenton Chemistry. J. Am. Chem. Soc. 2015, 137, 2867-2874. 
5. NMR spectra of the compounds

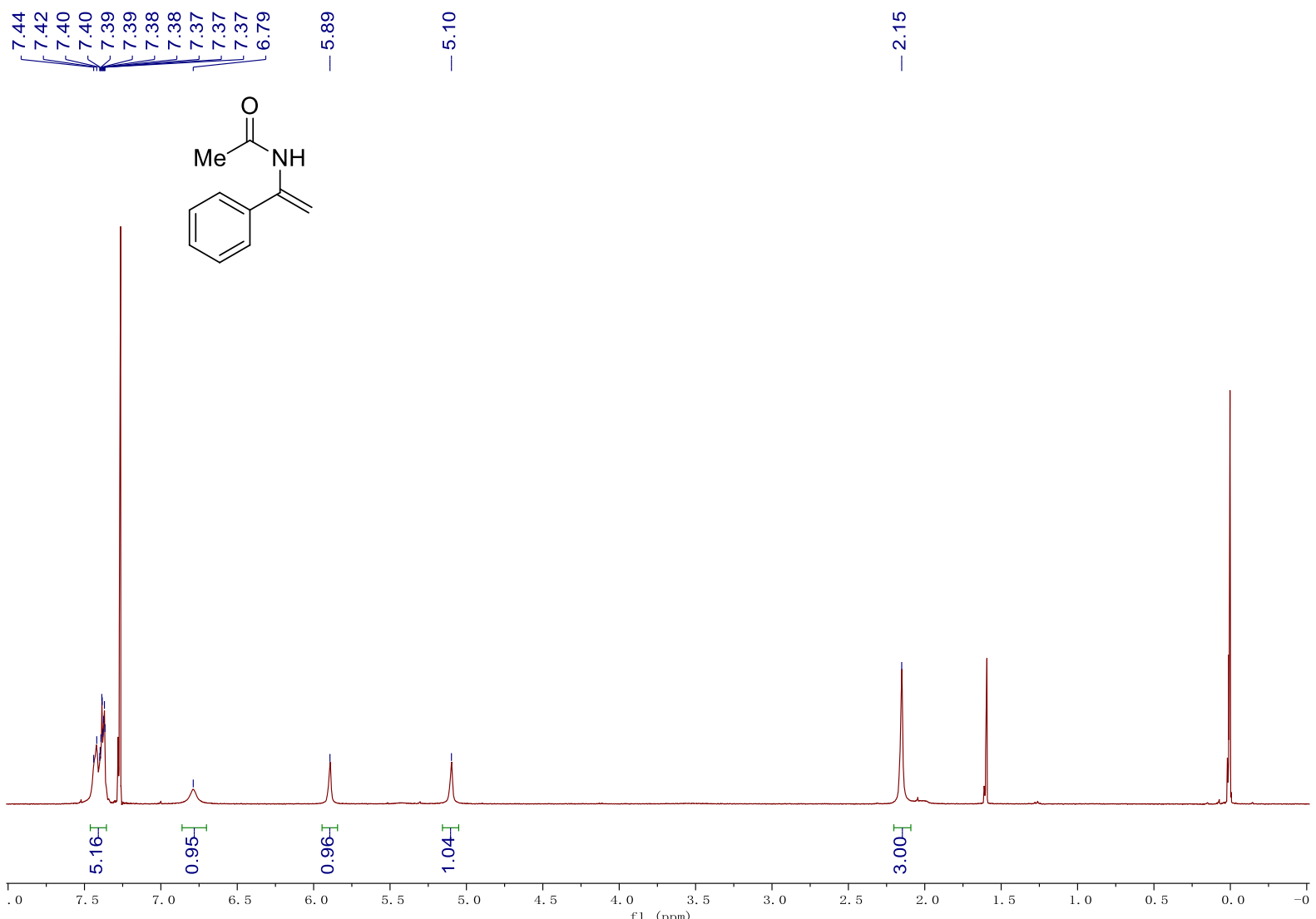

${ }^{1} \mathrm{H}$ NMR spectrum for compound $1 \mathrm{a}\left(\mathrm{CDCl}_{3}\right)$
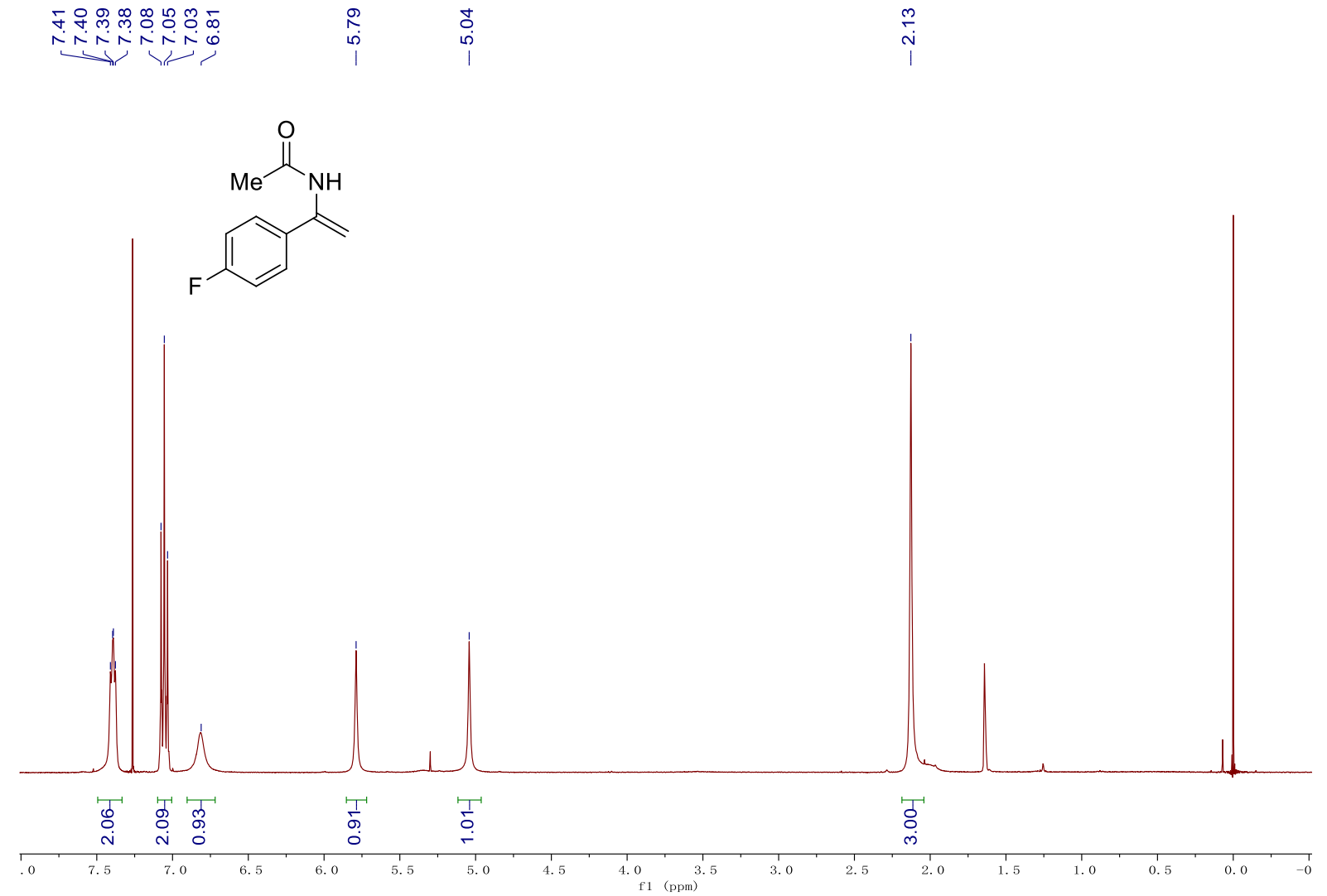

${ }^{\mathbf{1}} \mathrm{H}$ NMR spectrum for compound $1 \mathrm{~b}\left(\mathrm{CDCl}_{3}\right)$ 


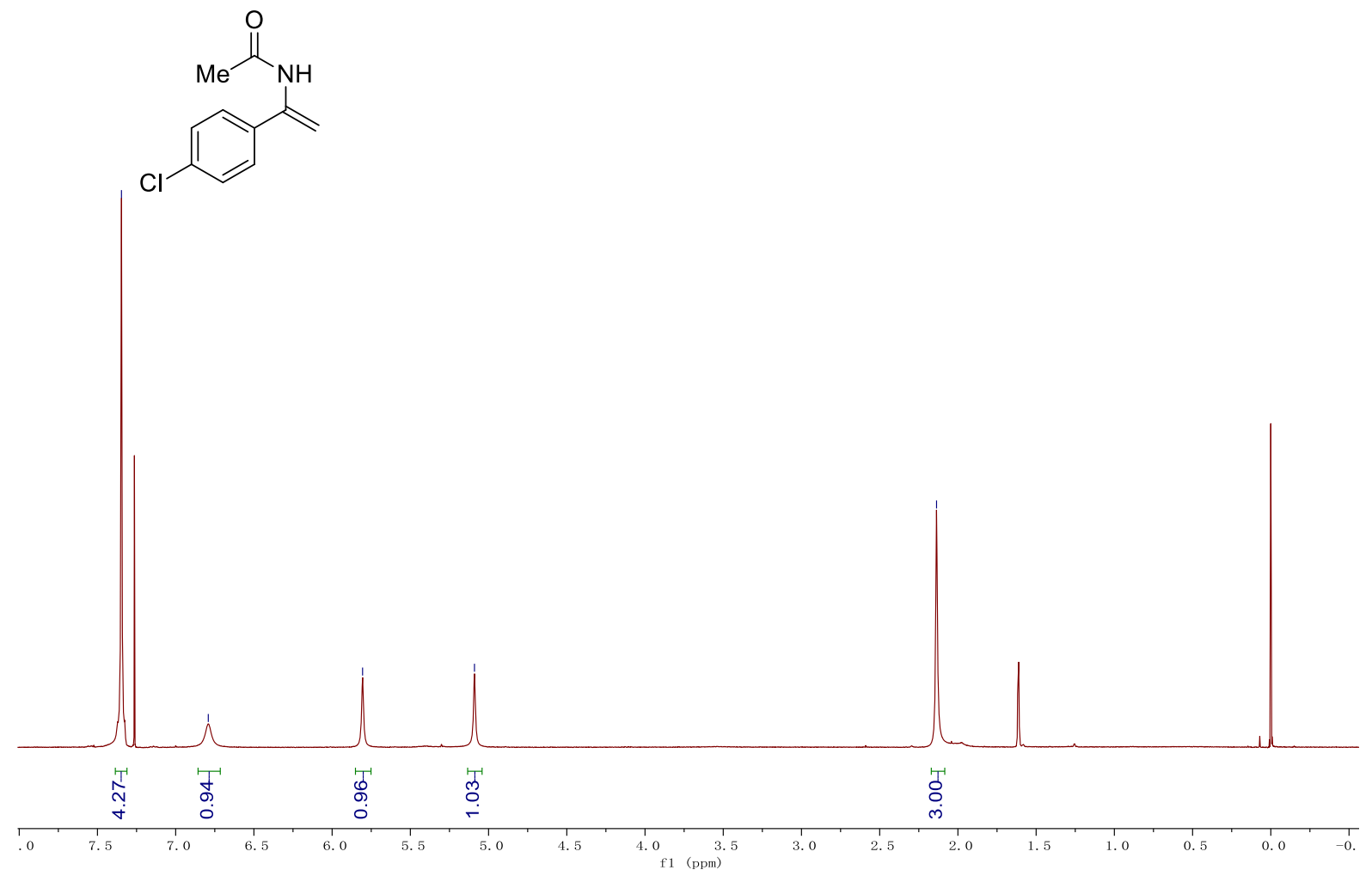

${ }^{1} \mathrm{H}$ NMR spectrum for compound $1 \mathrm{c}\left(\mathrm{CDCl}_{3}\right)$

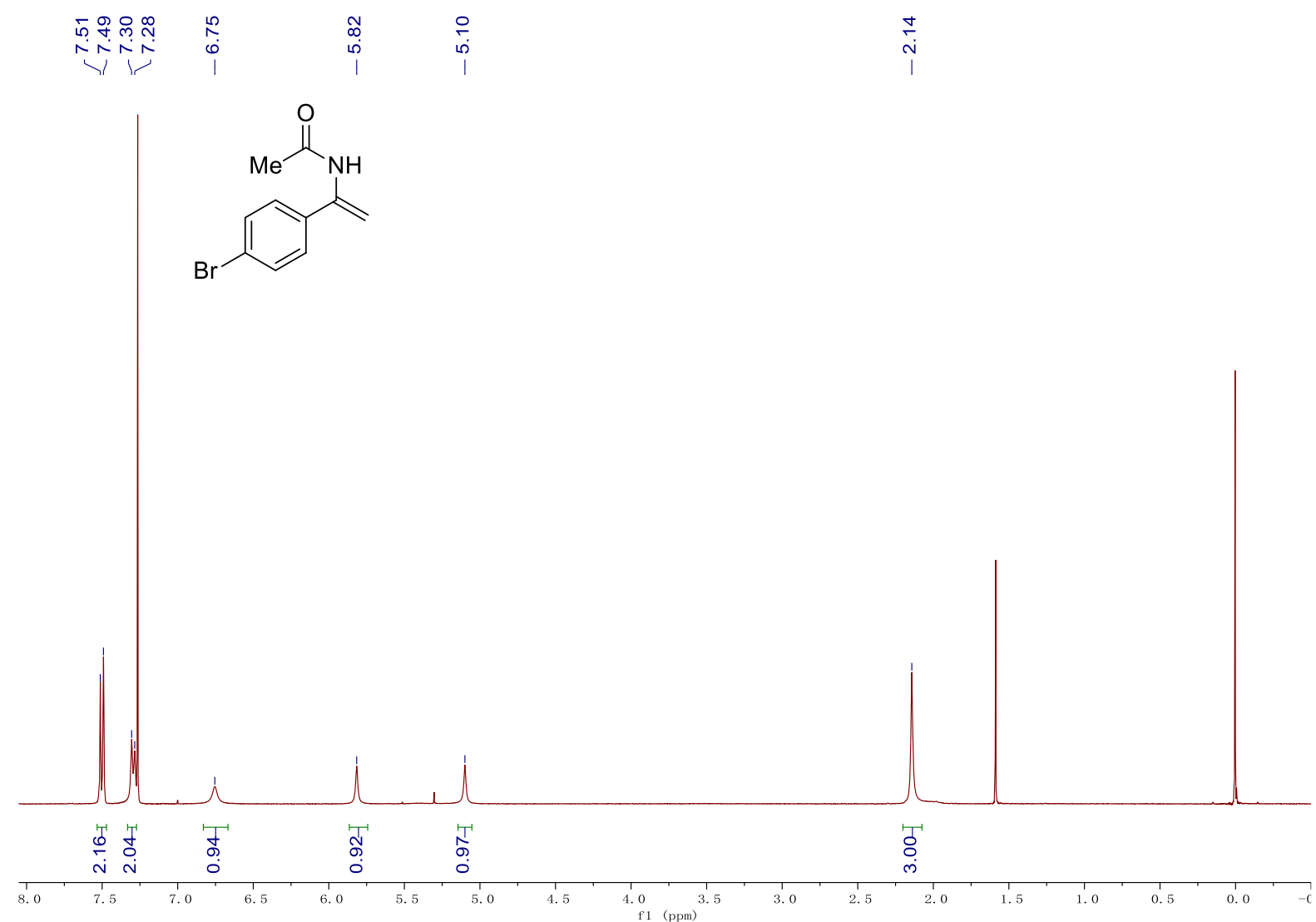

${ }^{1} \mathrm{H}$ NMR spectrum for compound $1 \mathrm{~d}\left(\mathrm{CDCl}_{3}\right)$ 


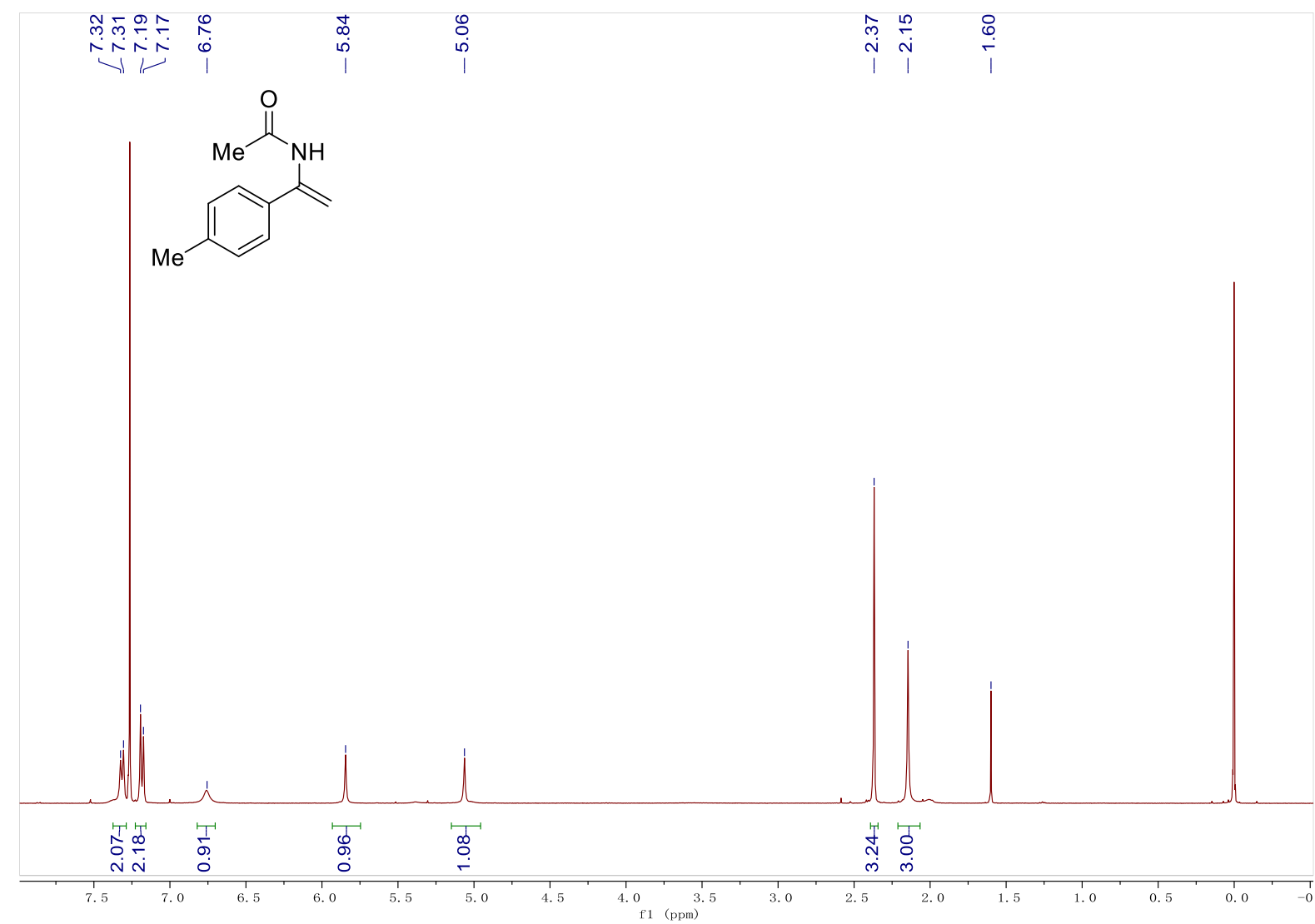

${ }^{1} \mathrm{H}$ NMR spectrum for compound 1e ( $\left.\mathrm{CDCl}_{3}\right)$

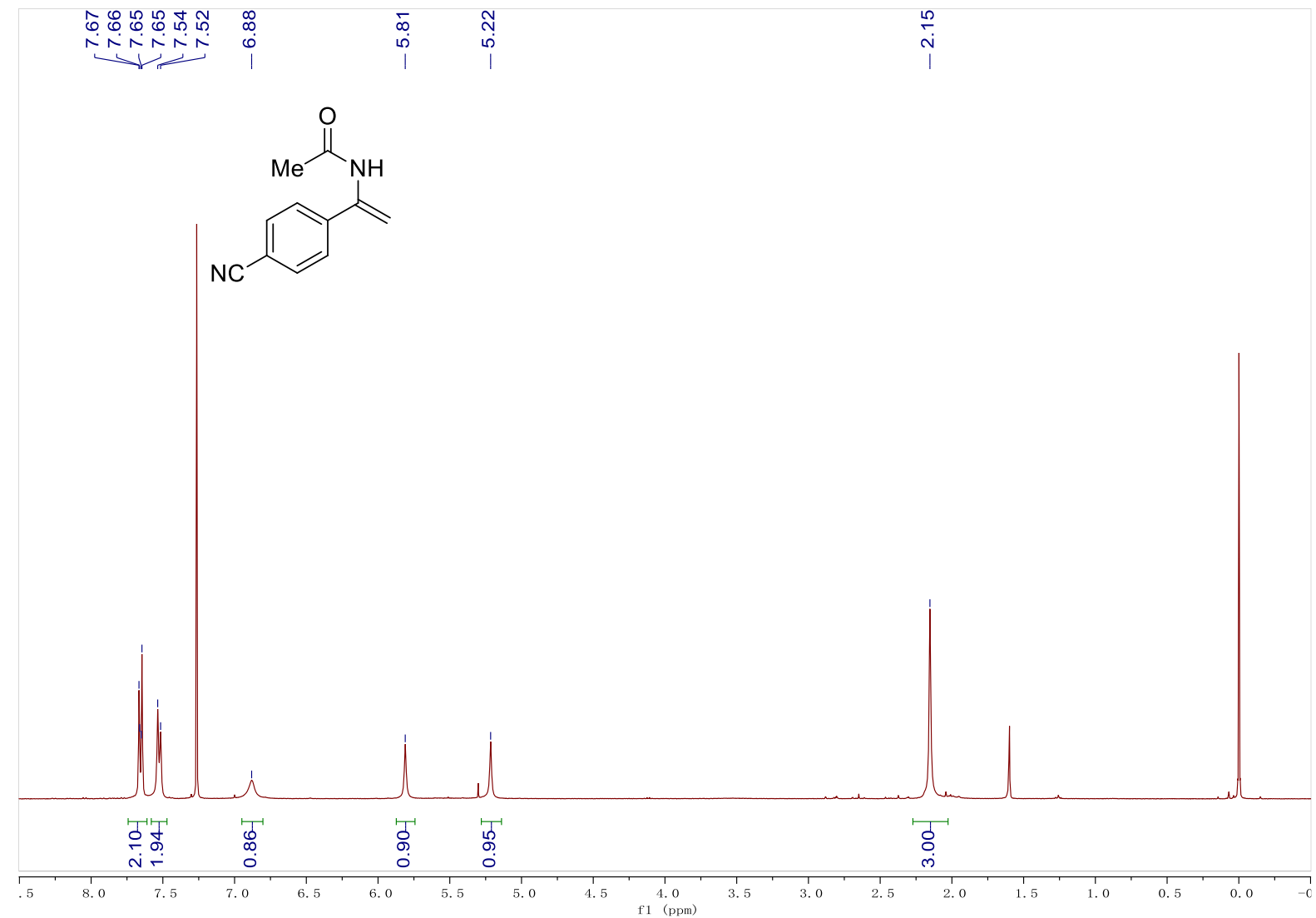

${ }^{1} \mathrm{H}$ NMR spectrum for compound $1 \mathrm{f}\left(\mathrm{CDCl}_{3}\right)$ 


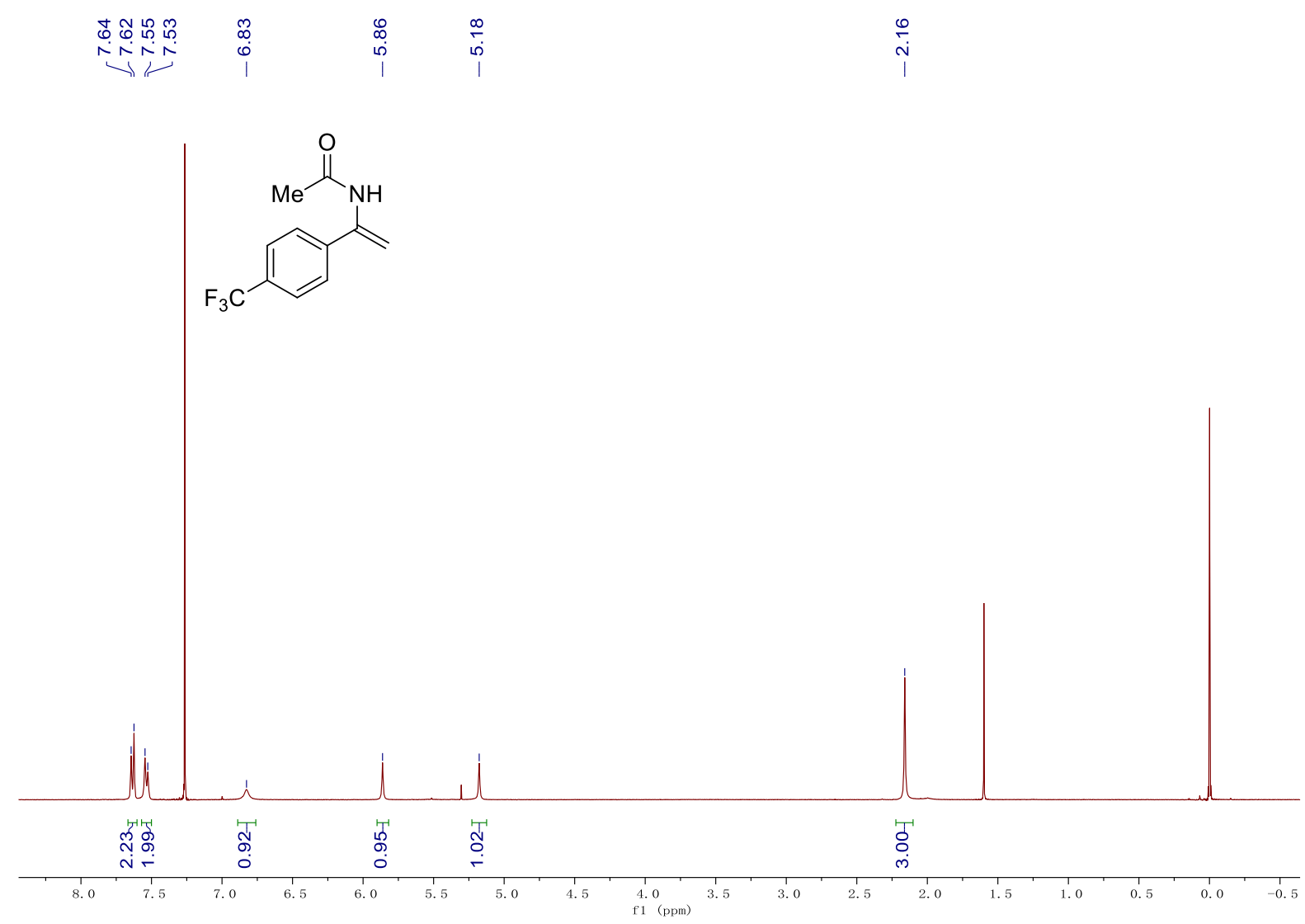

${ }^{1} \mathrm{H}$ NMR spectrum for compound $1 \mathrm{~g}\left(\mathrm{CDCl}_{3}\right)$

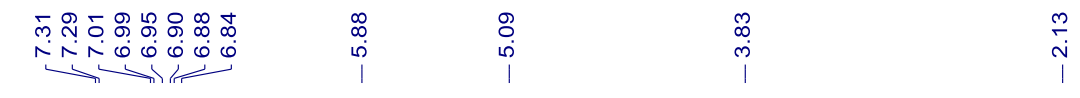

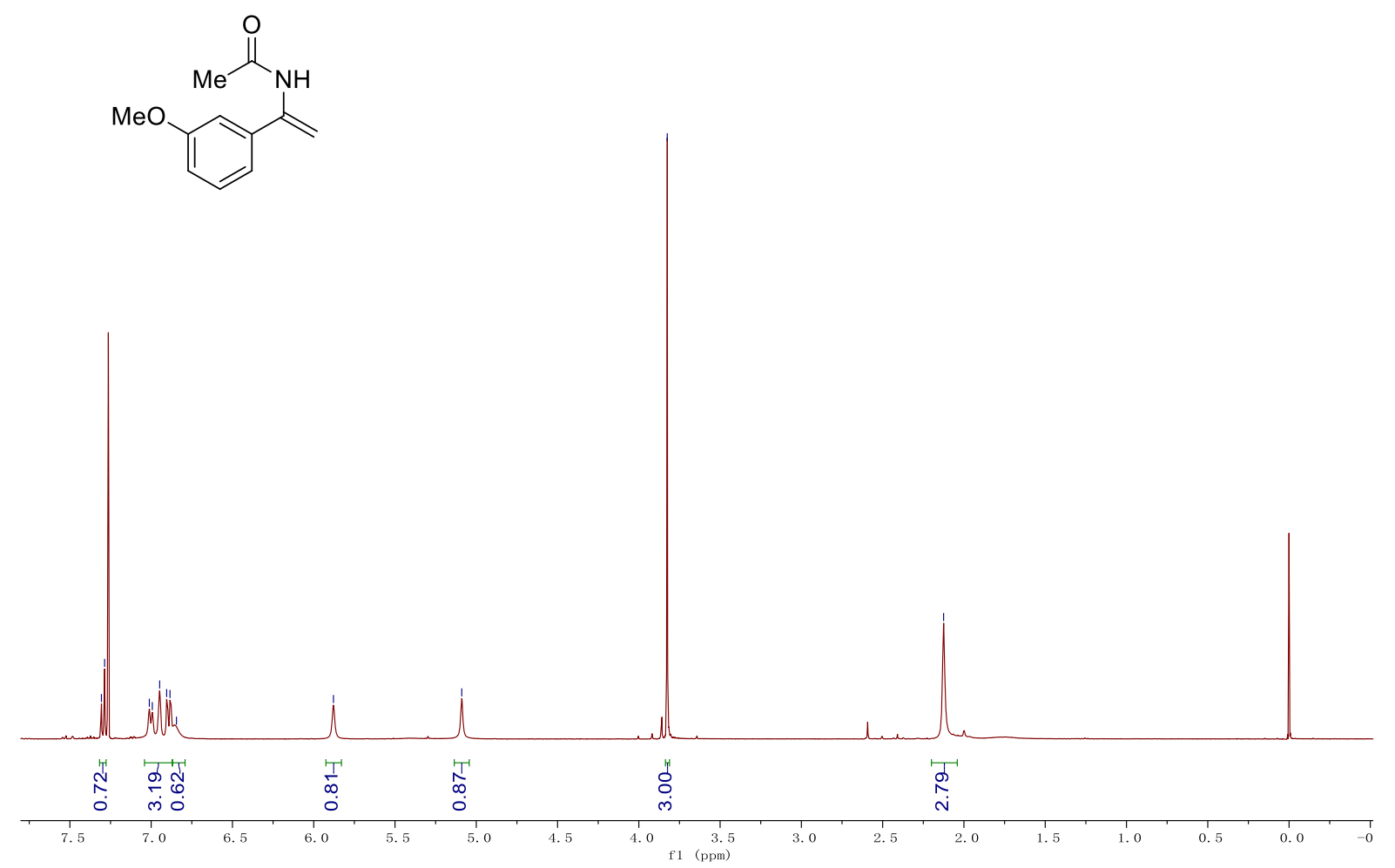

${ }^{1} \mathrm{H}$ NMR spectrum for compound $1 \mathrm{~h}\left(\mathrm{CDCl}_{3}\right)$ 


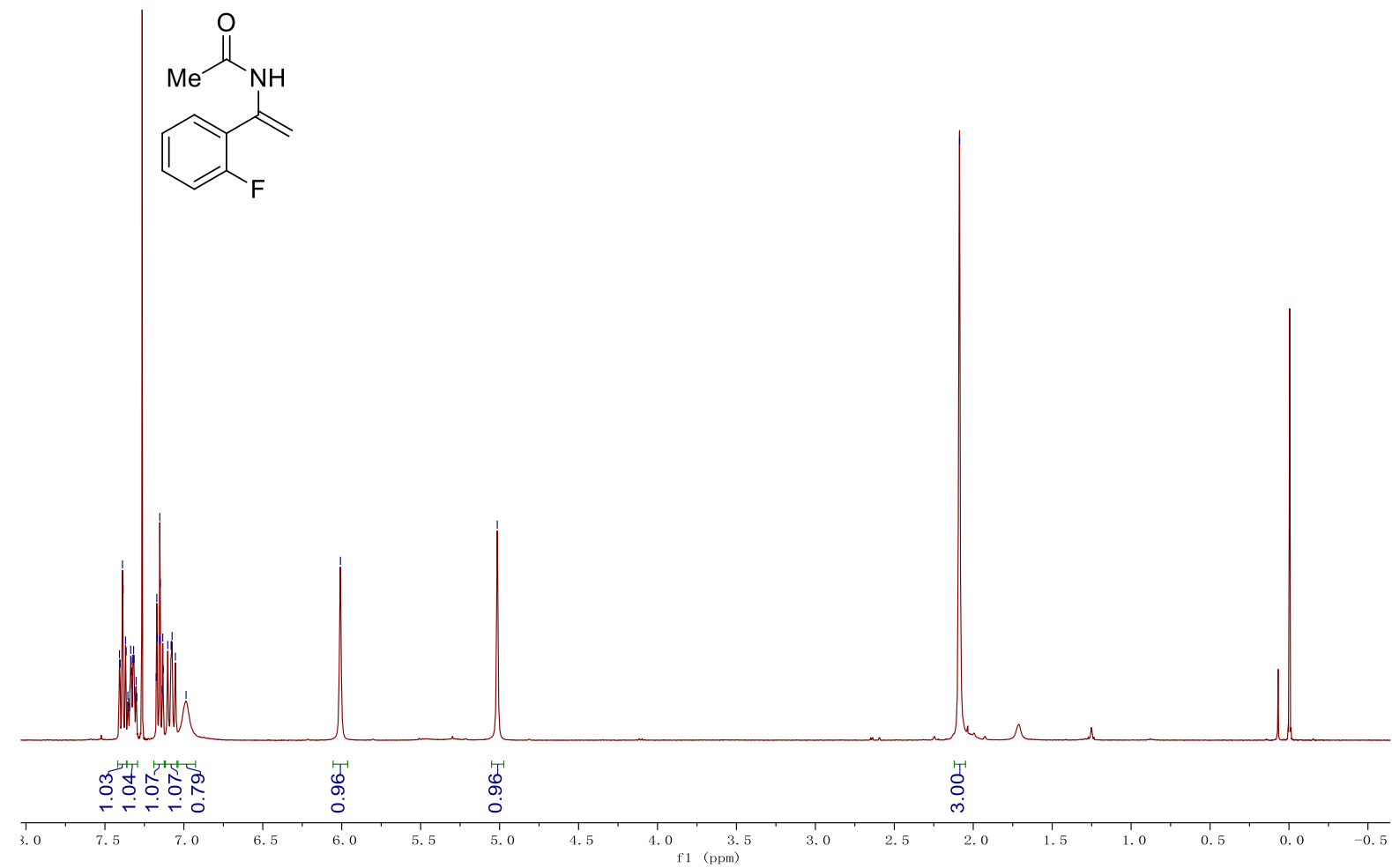

${ }^{1} \mathrm{H}$ NMR spectrum for compound $1 \mathrm{i}\left(\mathrm{CDCl}_{3}\right)$

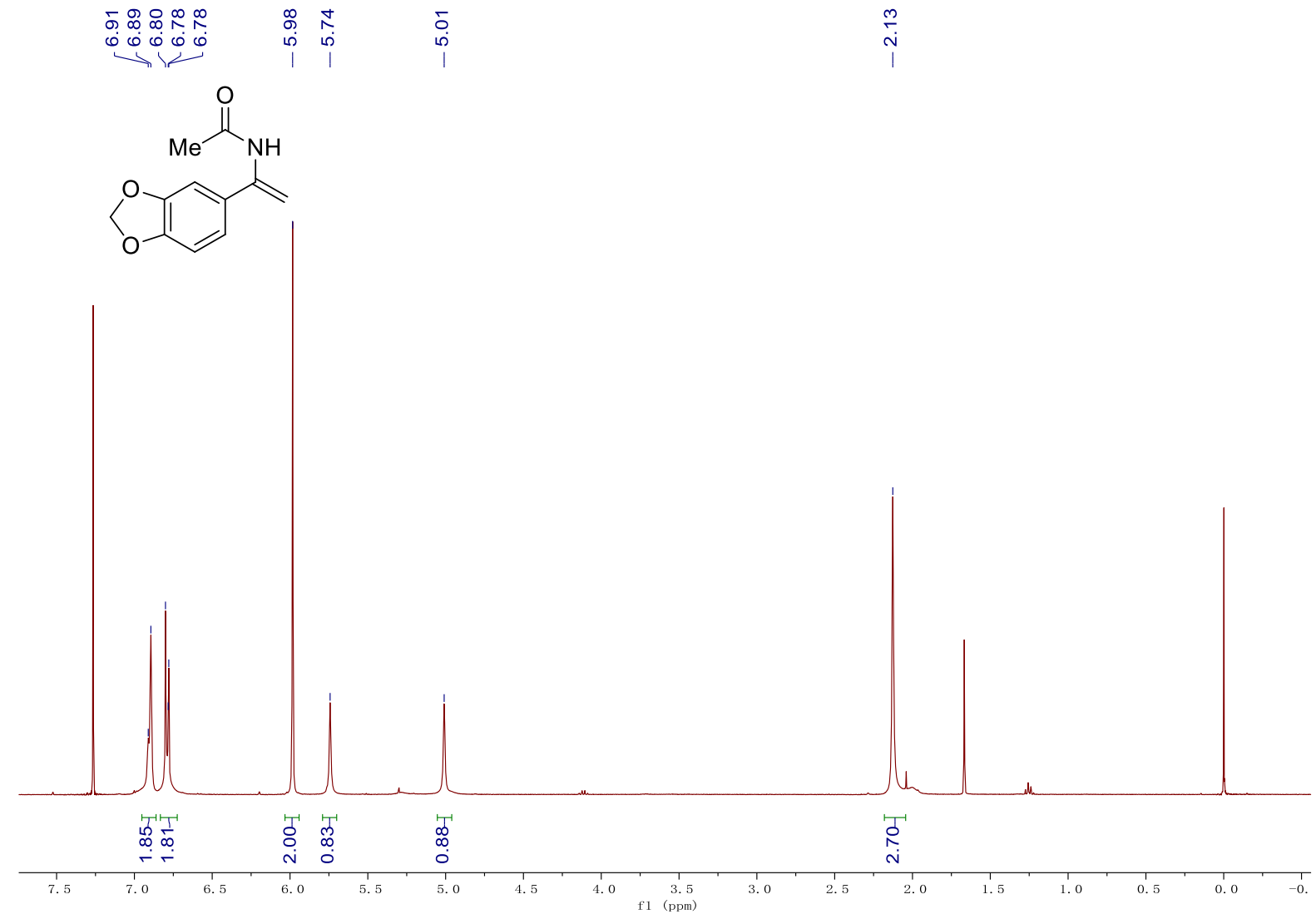

${ }^{1} \mathrm{H}$ NMR spectrum for compound $1 \mathrm{j}\left(\mathrm{CDCl}_{3}\right)$ 


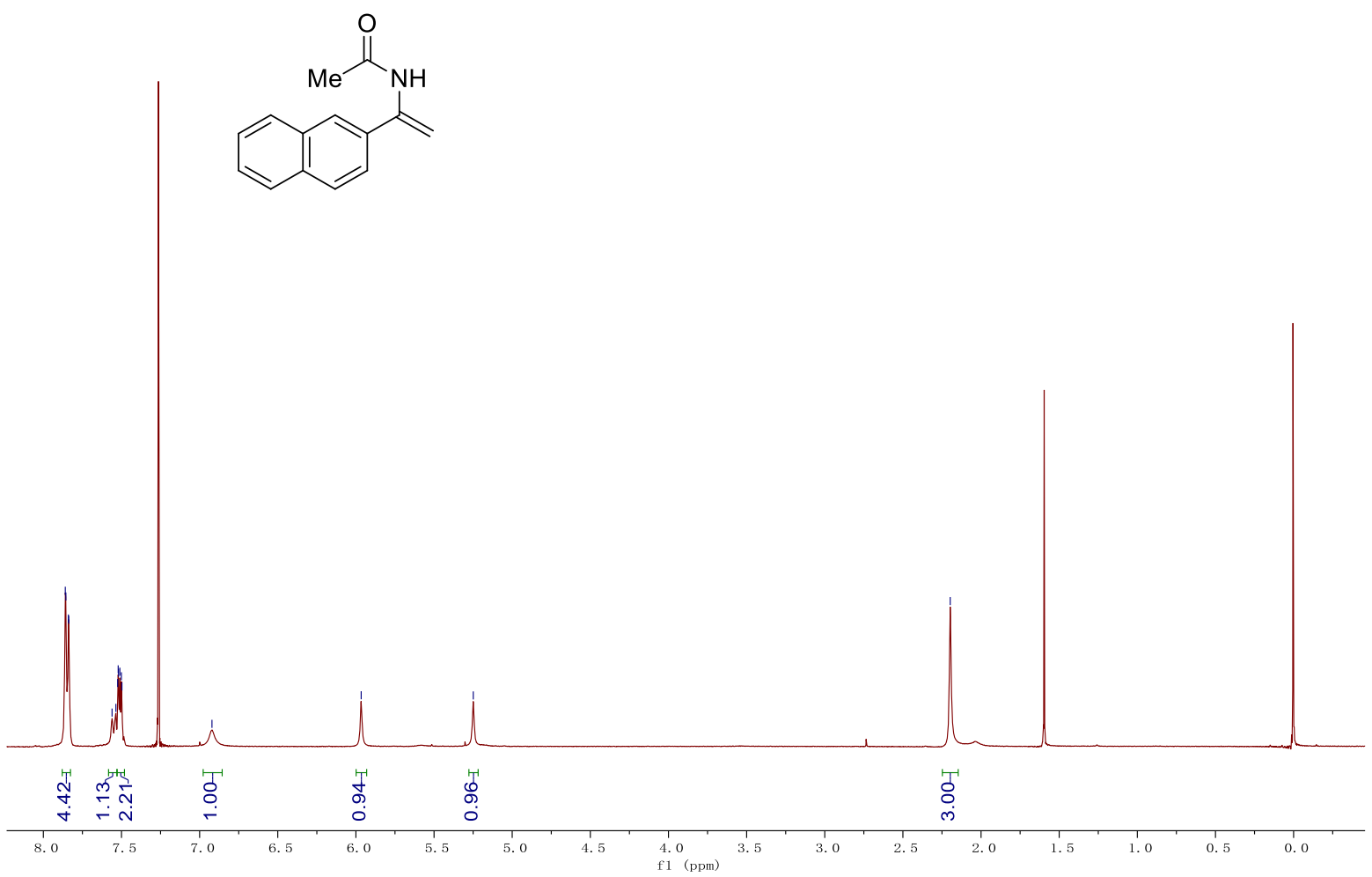

${ }^{1} \mathrm{H}$ NMR spectrum for compound $1 \mathrm{k}\left(\mathrm{CDCl}_{3}\right)$

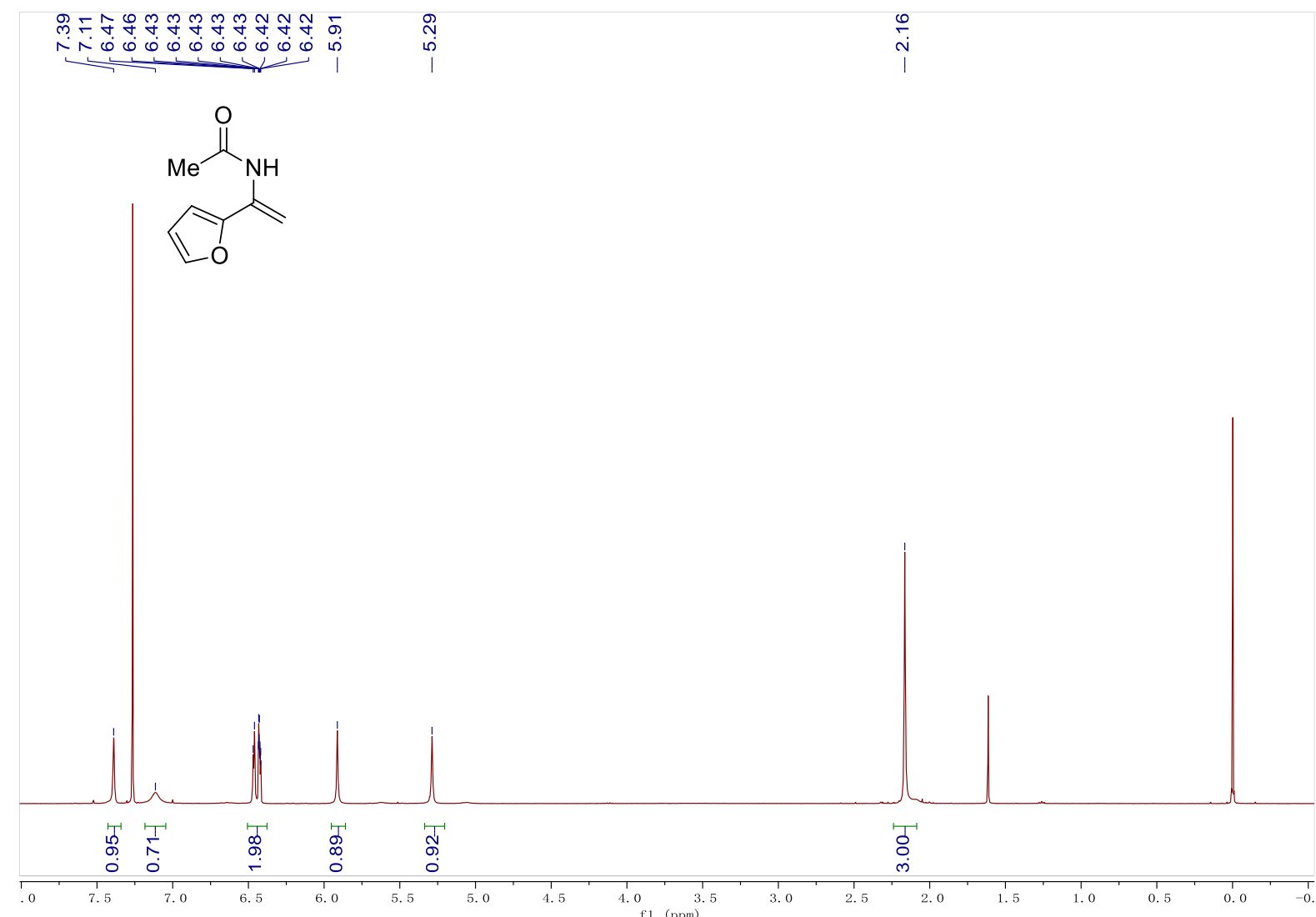

${ }^{1} \mathrm{H}$ NMR spectrum for compound $11\left(\mathrm{CDCl}_{3}\right)$ 


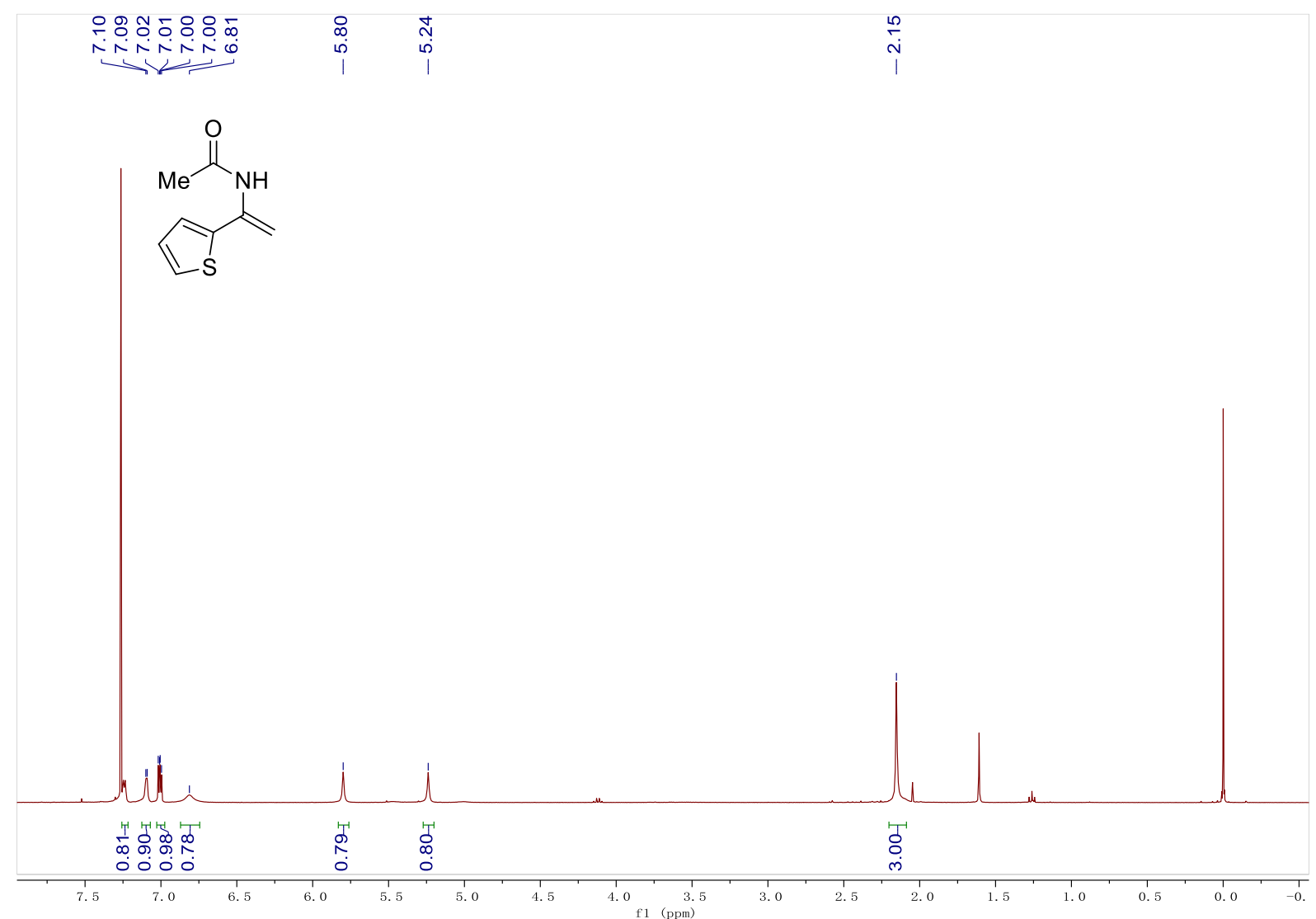

${ }^{1} \mathrm{H}$ NMR spectrum for compound $1 \mathrm{~m}\left(\mathrm{CDCl}_{3}\right)$

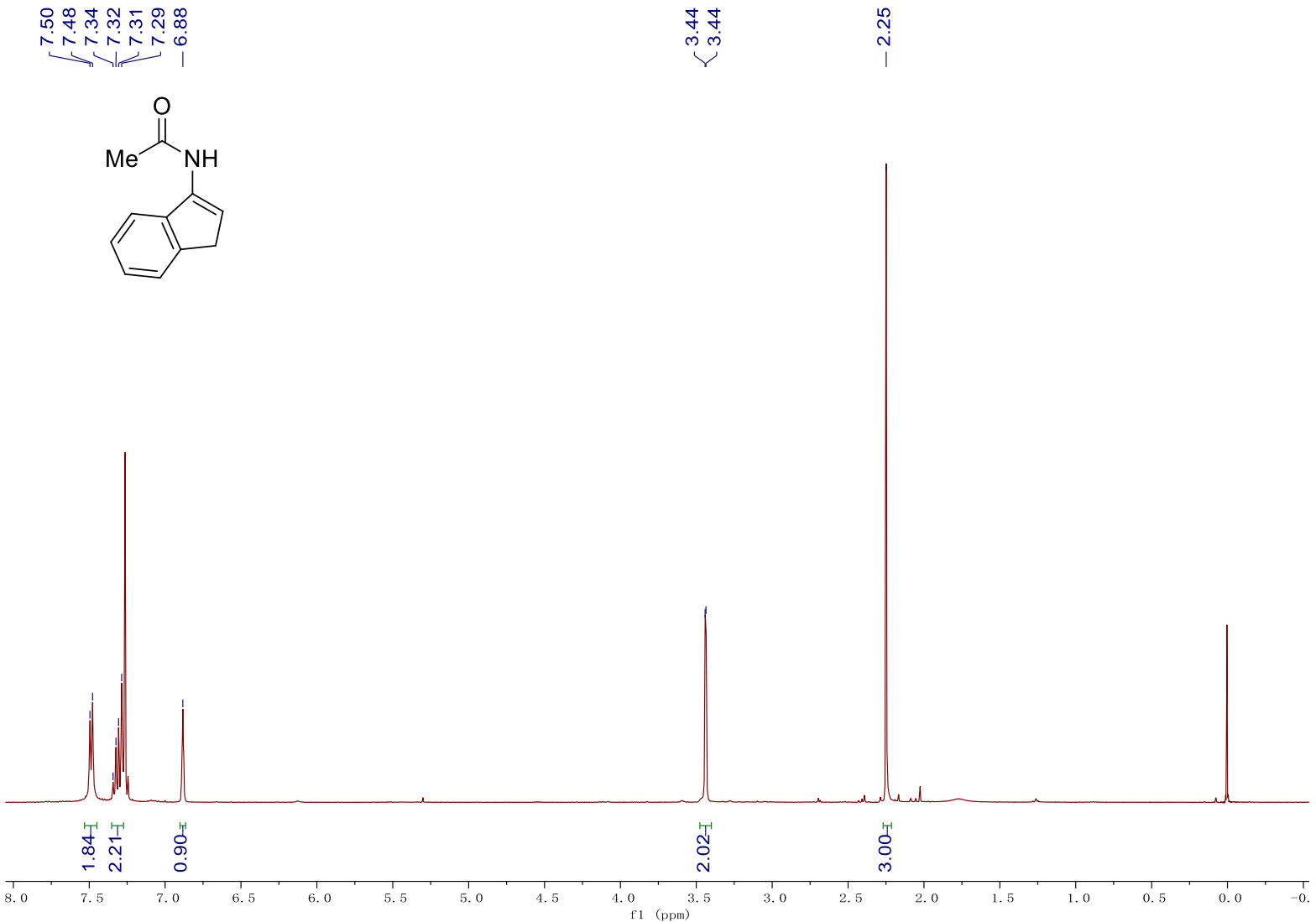

${ }^{1} \mathrm{H}$ NMR spectrum for compound $1 \mathrm{n}\left(\mathrm{CDCl}_{3}\right)$ 


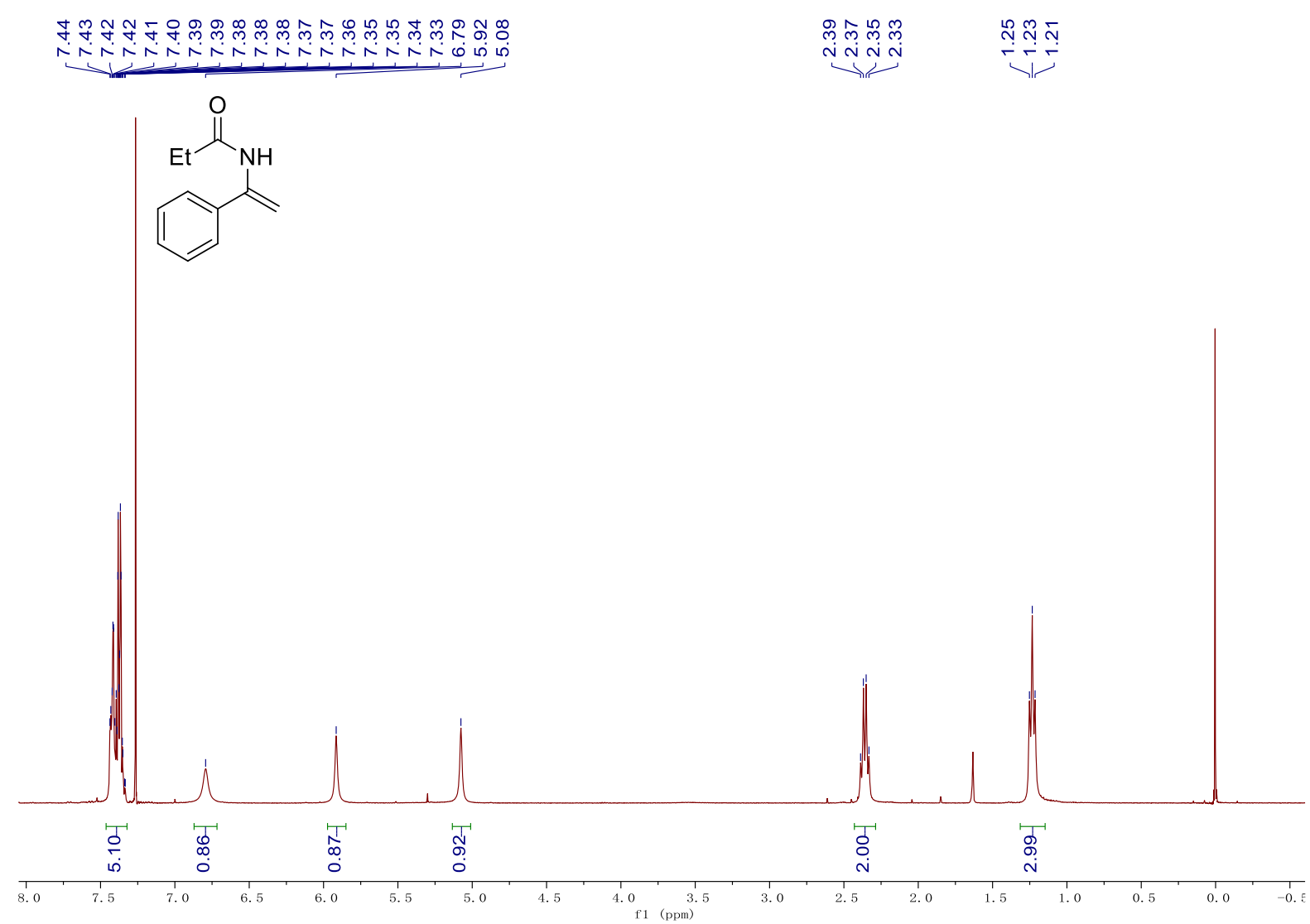

${ }^{1} \mathrm{H}$ NMR spectrum for compound $10\left(\mathrm{CDCl}_{3}\right)$

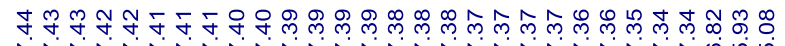

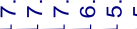

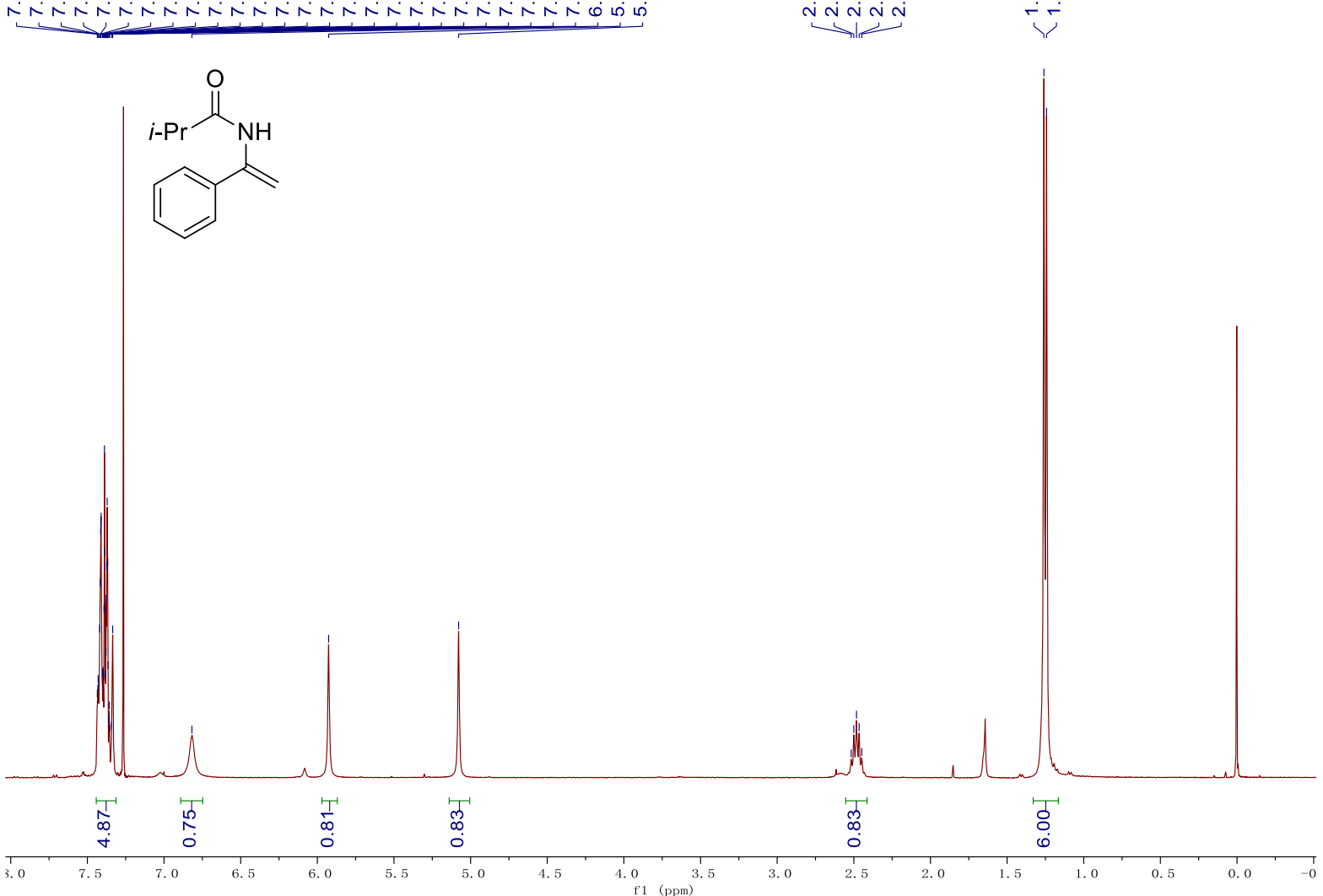

${ }^{1} \mathrm{H}$ NMR spectrum for compound $1 \mathrm{p}\left(\mathrm{CDCl}_{3}\right)$ 


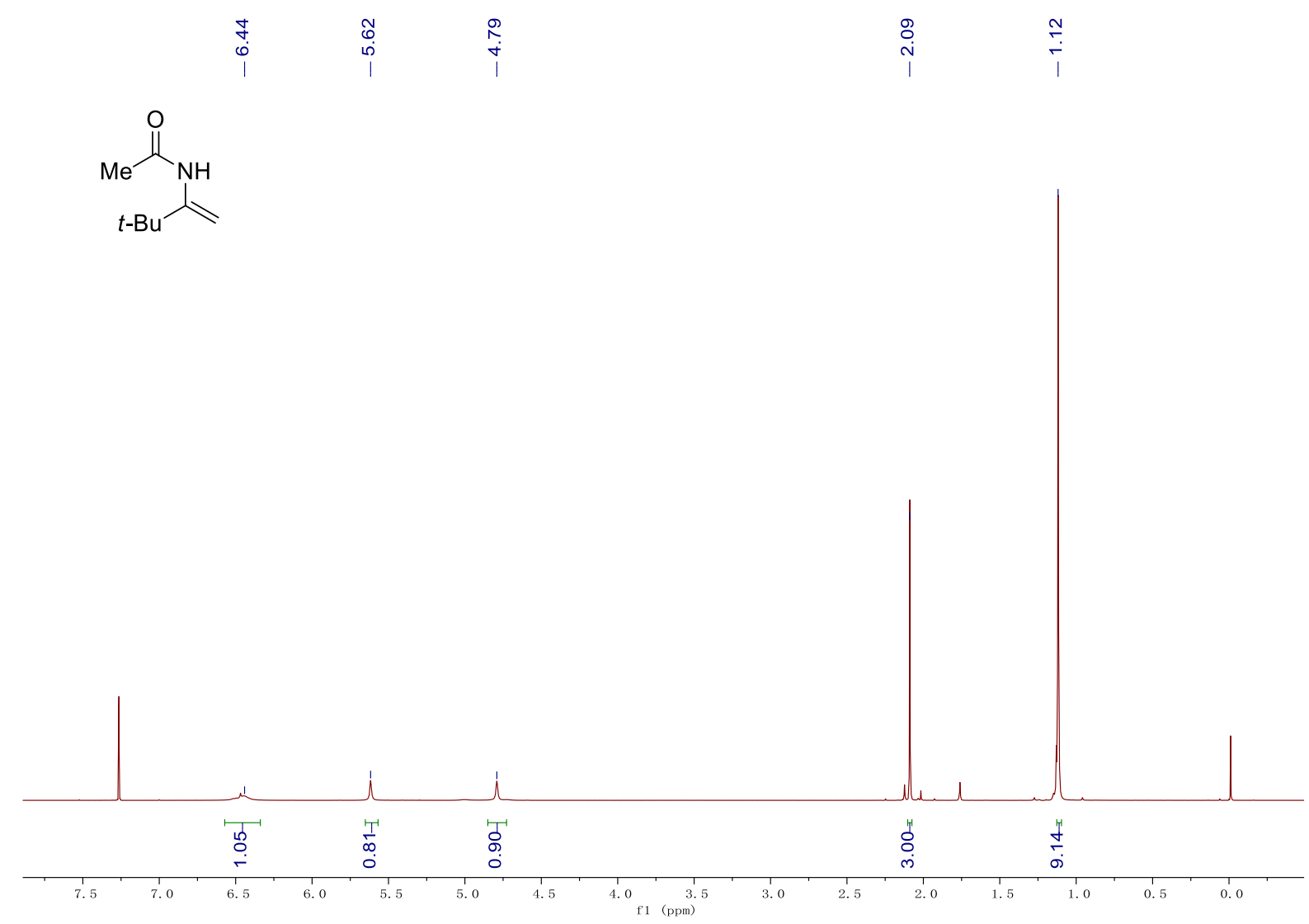

${ }^{1} \mathrm{H}$ NMR spectrum for compound $1 \mathrm{q}\left(\mathrm{CDCl}_{3}\right)$

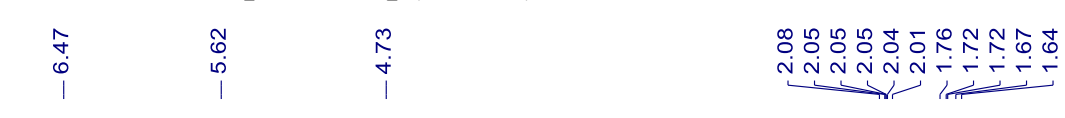

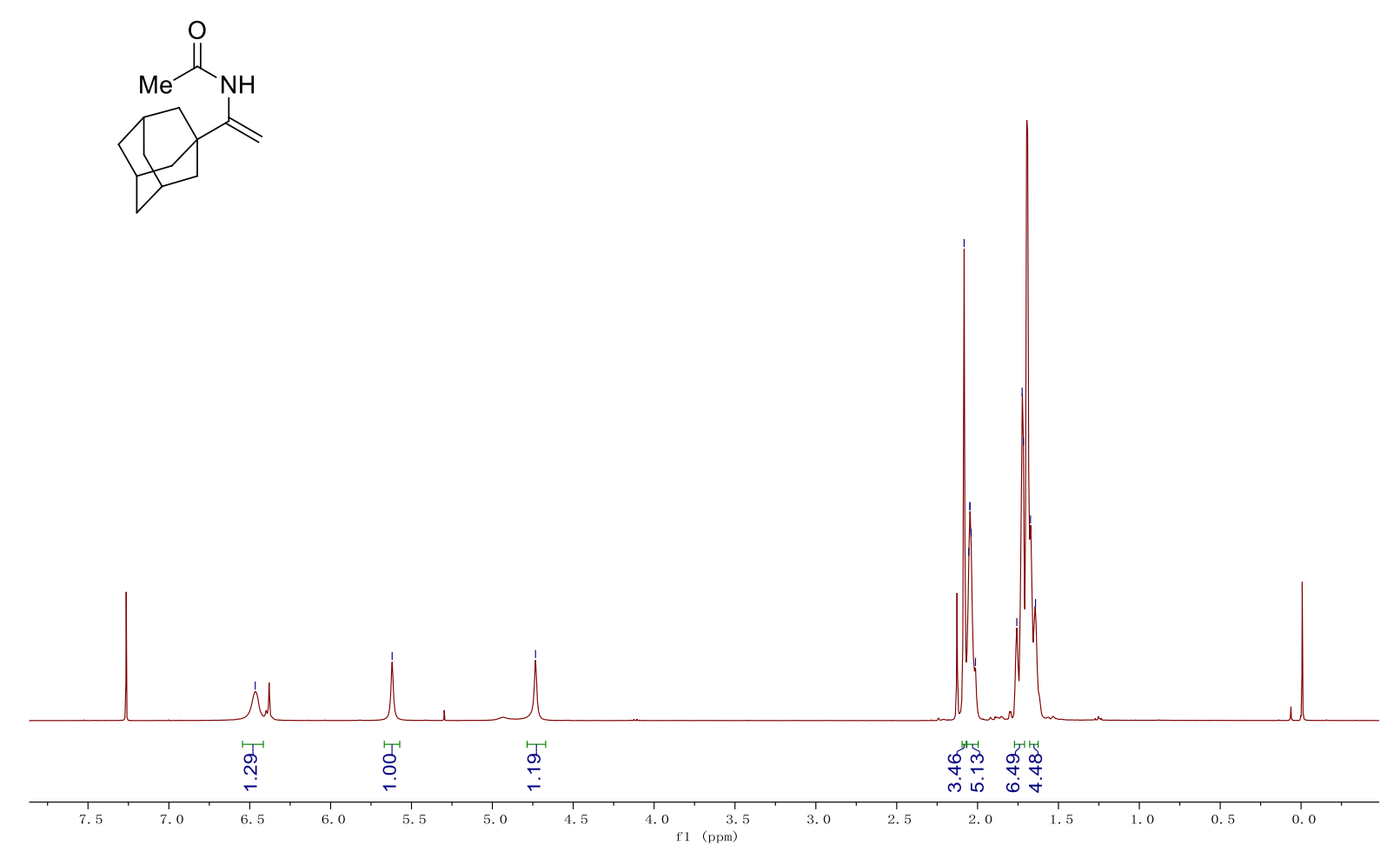

${ }^{1} \mathrm{H}$ NMR spectrum for compound $\operatorname{1r}\left(\mathrm{CDCl}_{3}\right)$ 


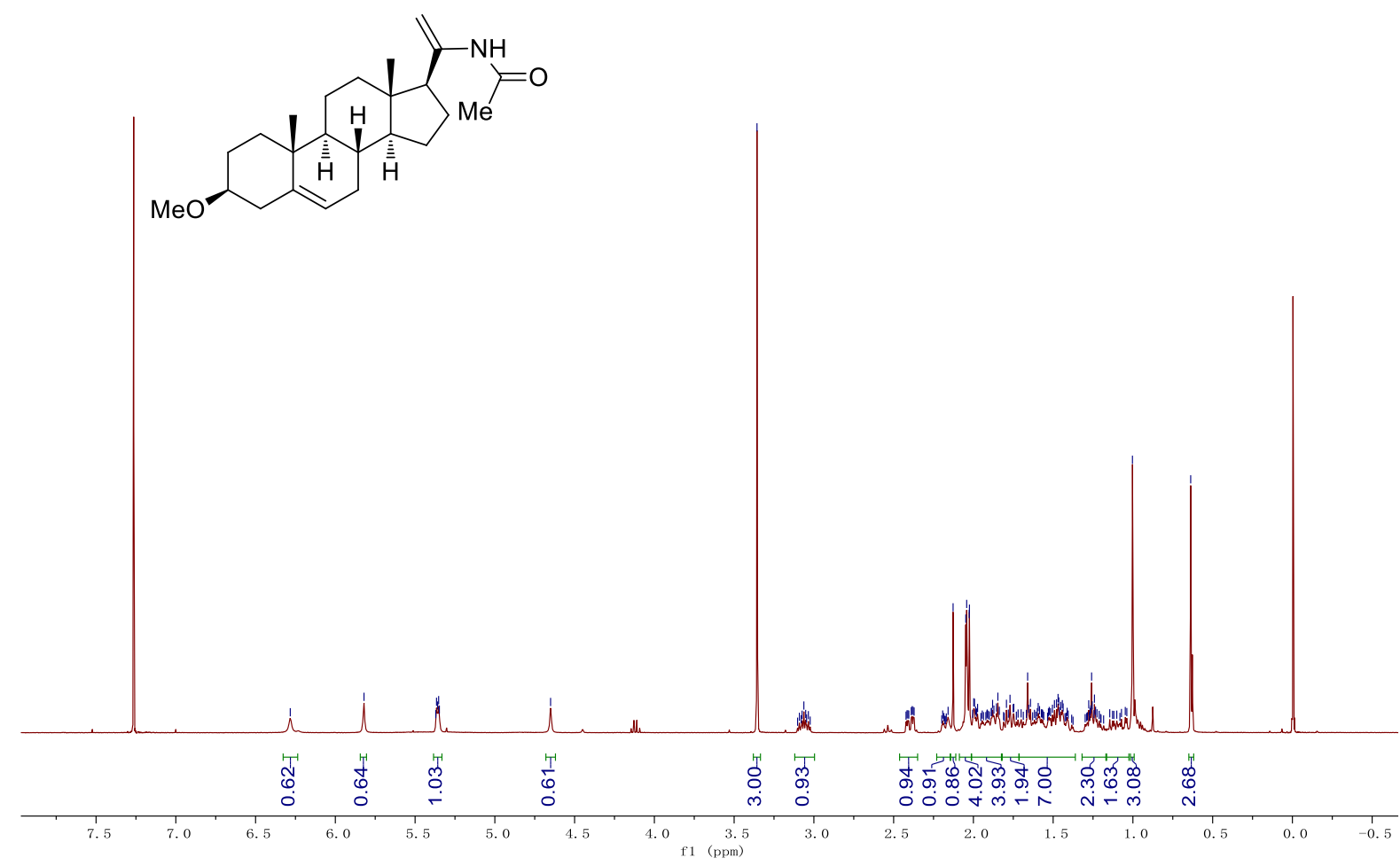

${ }^{1} \mathrm{H}$ NMR spectrum for compound $1 \mathrm{~s}\left(\mathrm{CDCl}_{3}\right)$

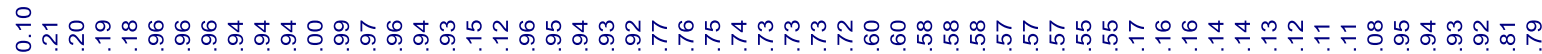
$\underbrace{0}$<smiles>CC1CC[C@H](C(C)C)[C@H](OC(=O)c2ccc(C=O)cc2)C1</smiles>

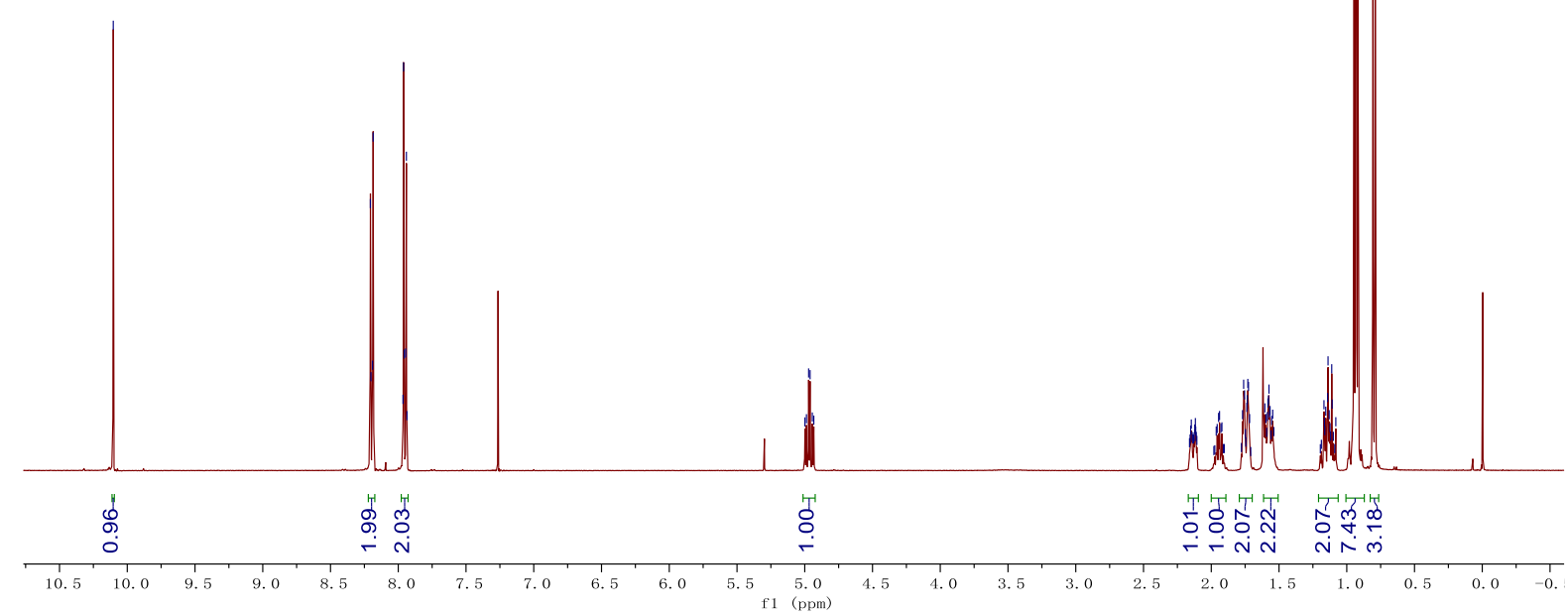

${ }^{1} \mathrm{H}$ NMR spectrum for compound 2ab $\left(\mathrm{CDCl}_{3}\right)$ 


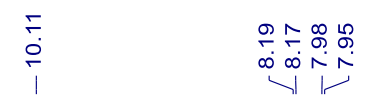

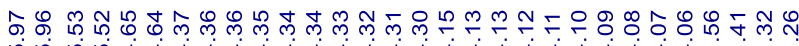

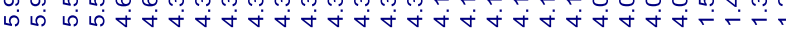<smiles>CC1(C)OCC([C@H]2OC3OC(C)(C)O[C@@H]3[C@H]2OC(=O)c2ccc(C=O)cc2)O1</smiles> 


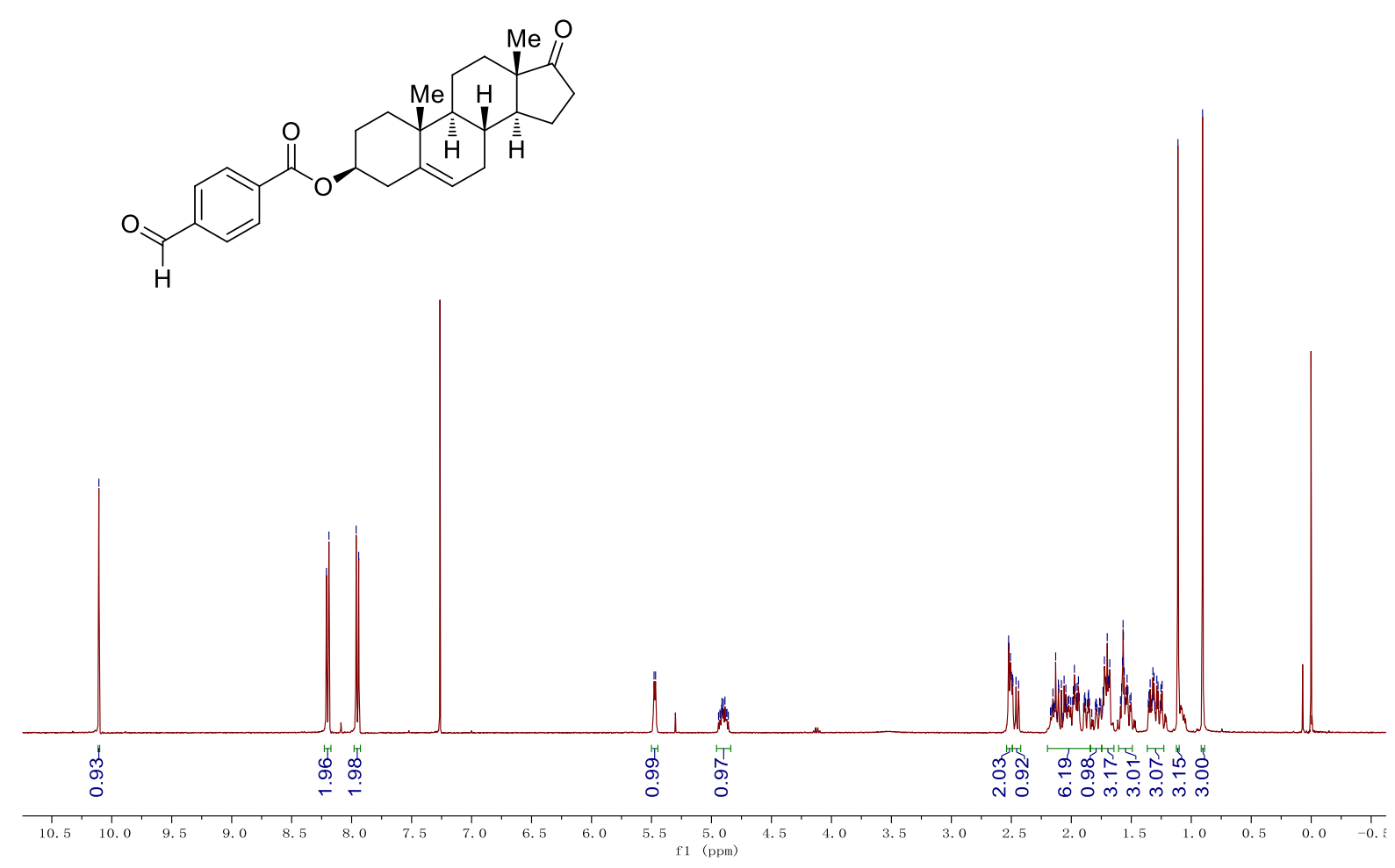

${ }^{1} \mathrm{H}$ NMR spectrum for compound 2ae $\left(\mathrm{CDCl}_{3}\right)$ 

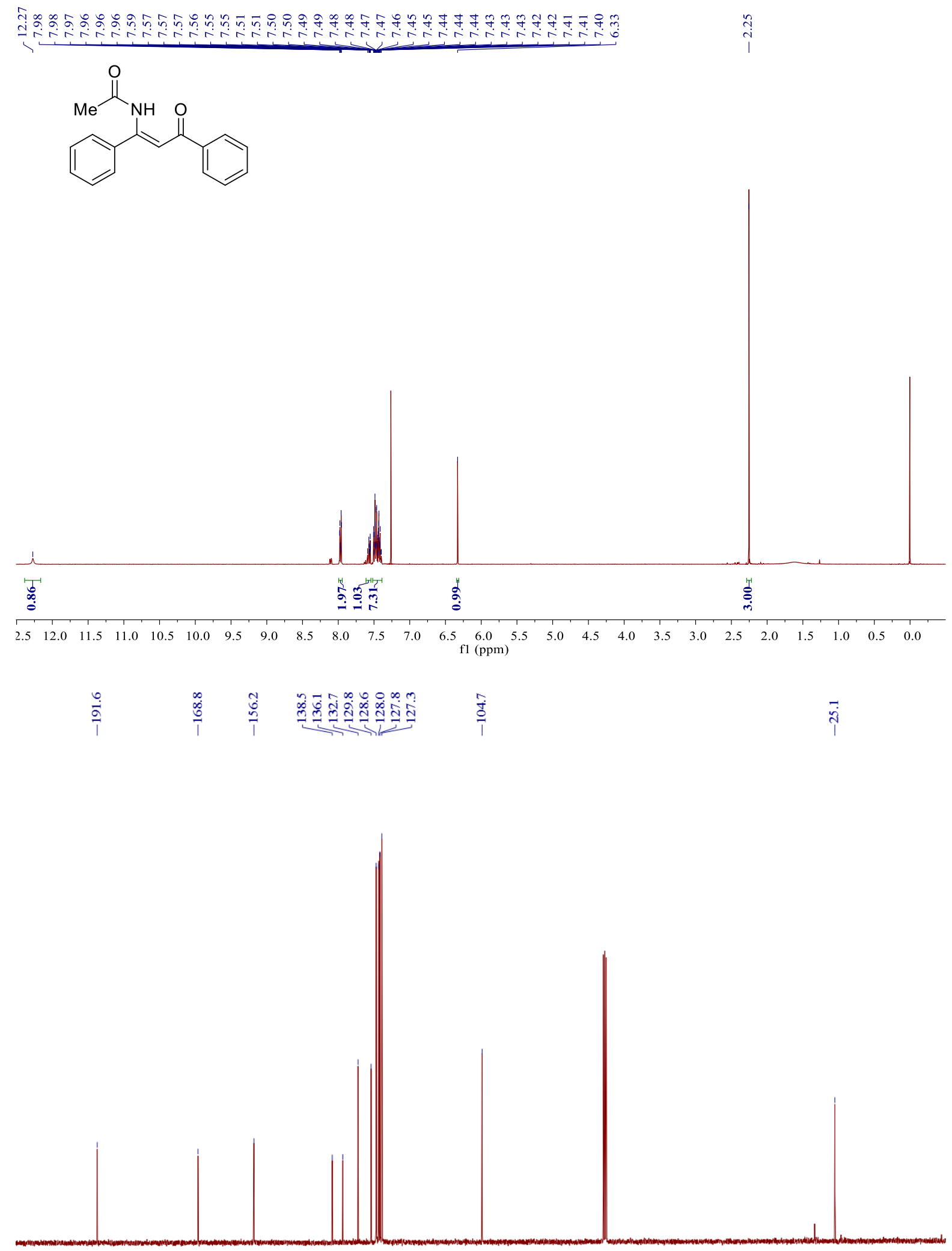

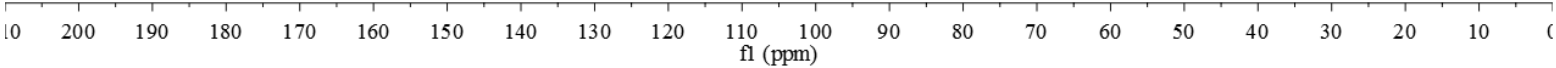

${ }^{1} \mathrm{H}$ and ${ }^{13} \mathrm{C}$ NMR spectra for product $3 \mathrm{a}\left(\mathrm{CDCl}_{3}\right)$ 


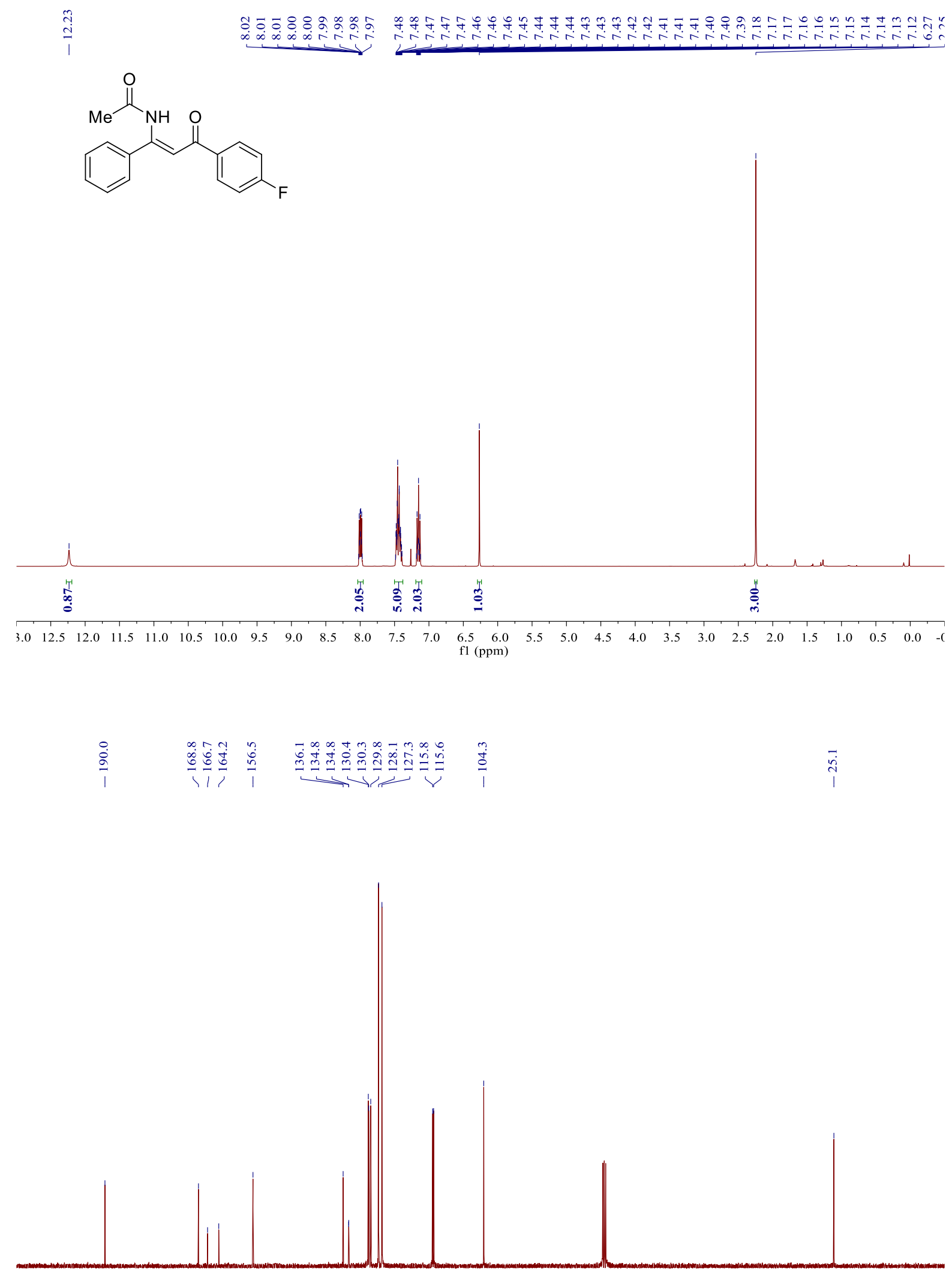

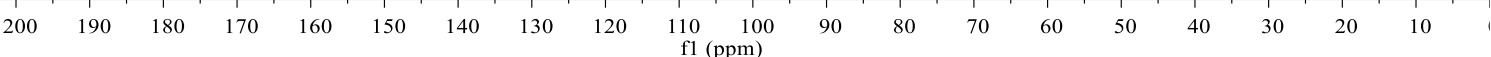




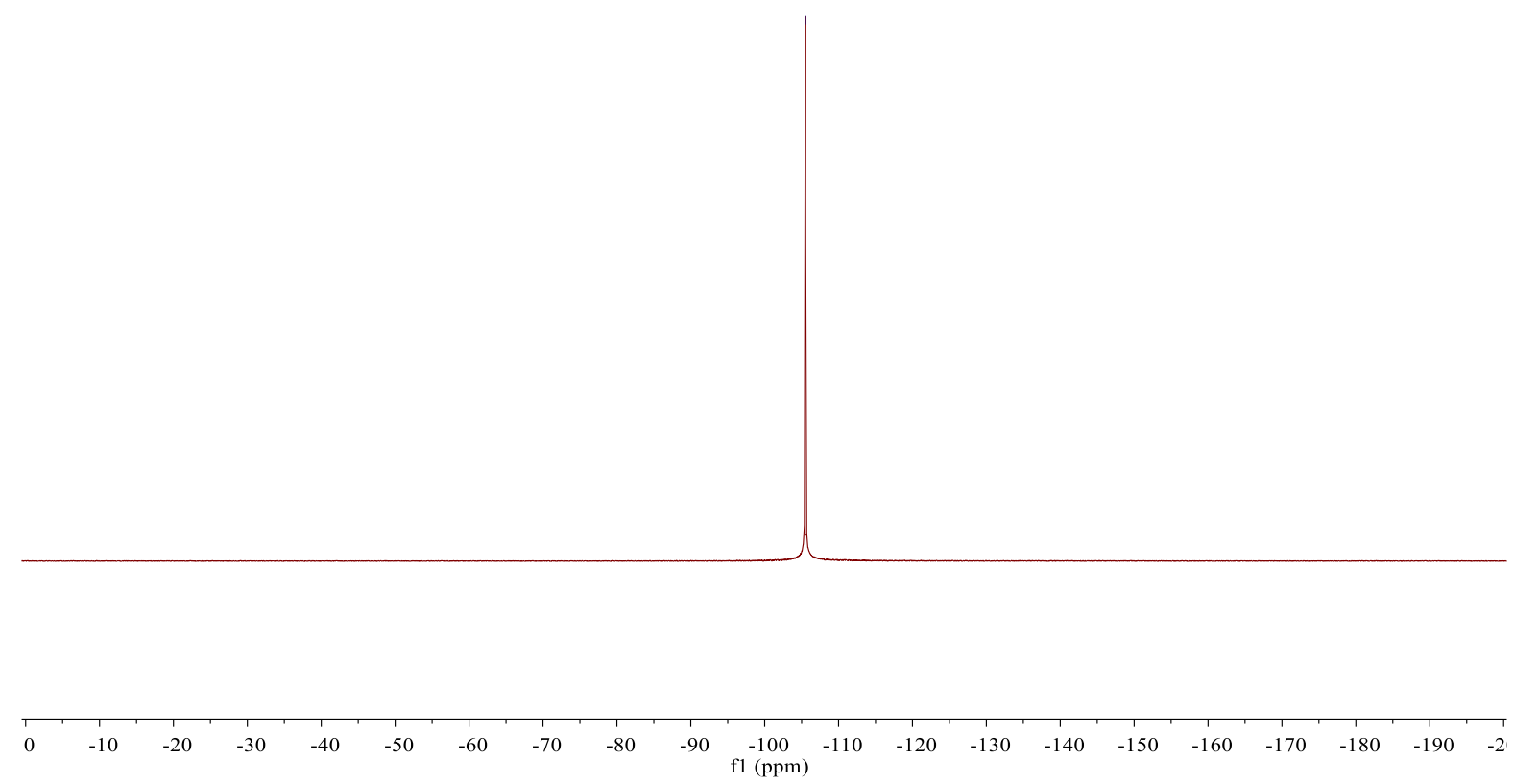

${ }^{1} \mathrm{H},{ }^{19} \mathrm{~F}$, and ${ }^{13} \mathrm{C}$ NMR spectra for product $3 \mathrm{~b}\left(\mathrm{CDCl}_{3}\right)$ 


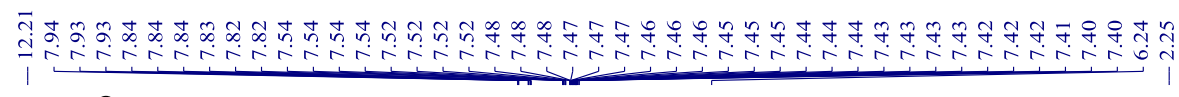<smiles>CC(=O)N/C(=C\C(=O)c1cccc(Cl)c1)c1ccccc1</smiles>

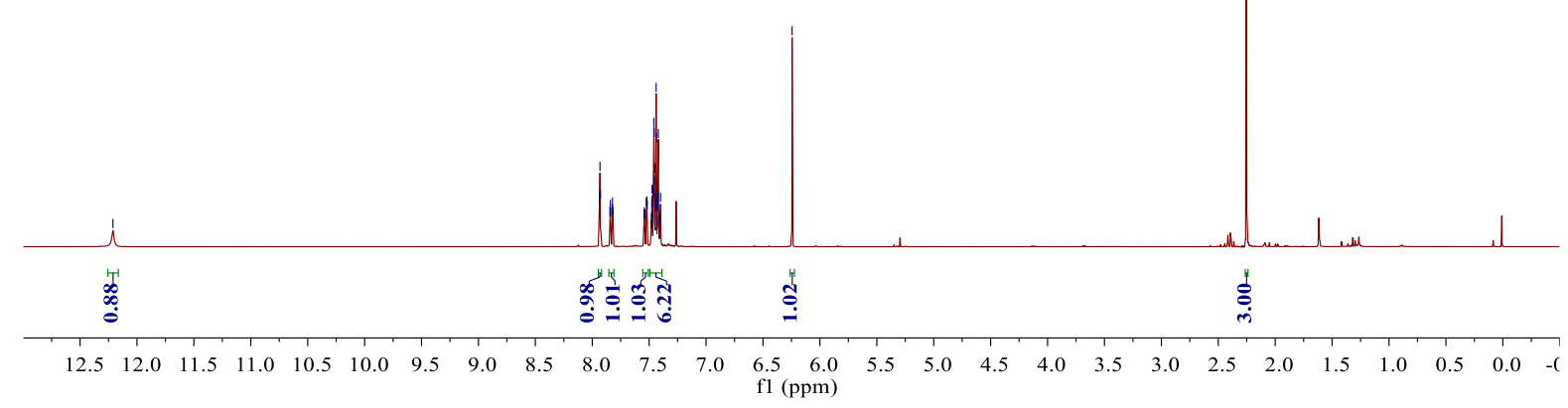

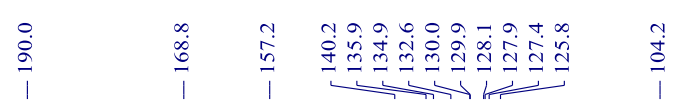

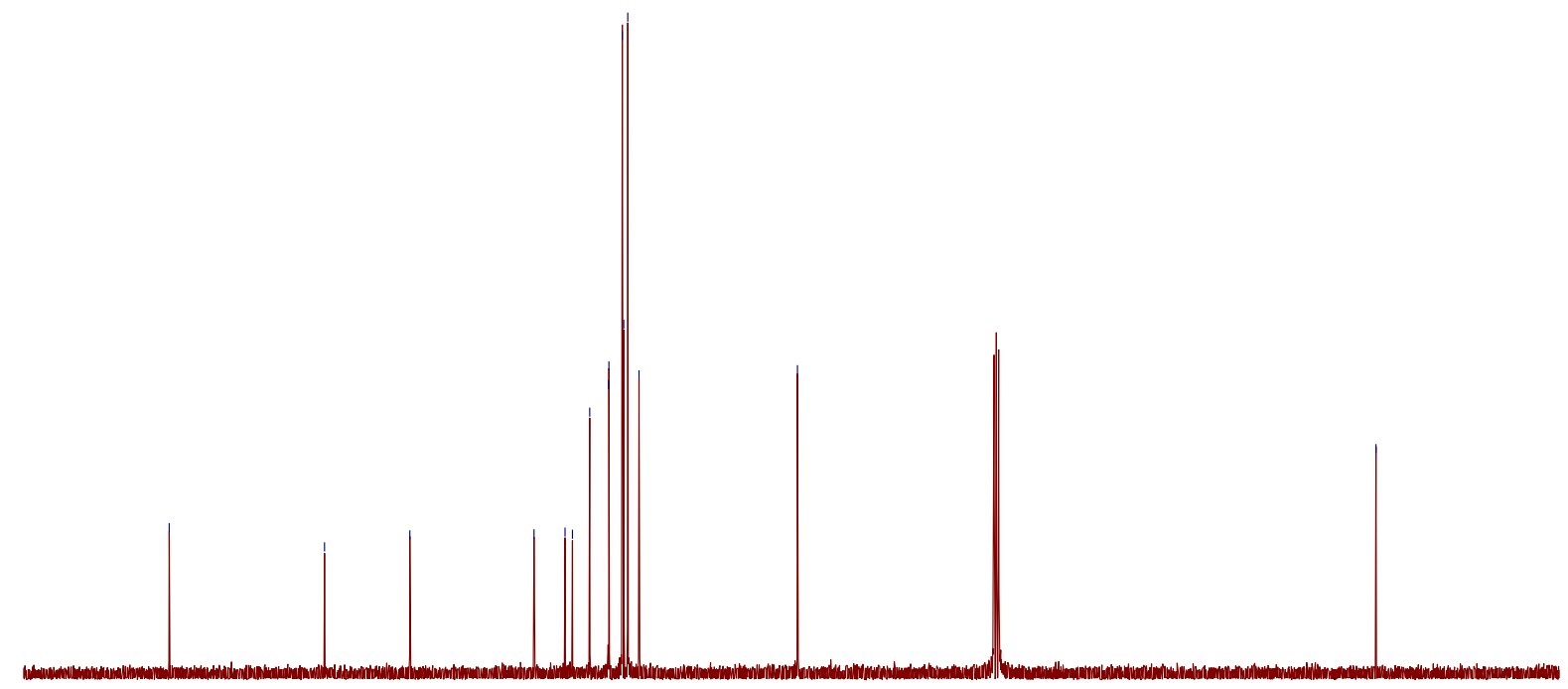

$\begin{array}{lllllllllll}200 & 190 & 180 & 170 & 160 & 150 & 140 & 130 & 120 & 110 \begin{array}{r}100 \\ \mathrm{fl}(\mathrm{ppm})\end{array}\end{array}$

${ }^{1} \mathrm{H}$ and ${ }^{13} \mathrm{C}$ NMR spectra for product $3 \mathrm{c}\left(\mathrm{CDCl}_{3}\right)$ 
<smiles>CC(=O)N/C(=C\C(=O)c1cccc(F)c1)c1ccccc1</smiles>

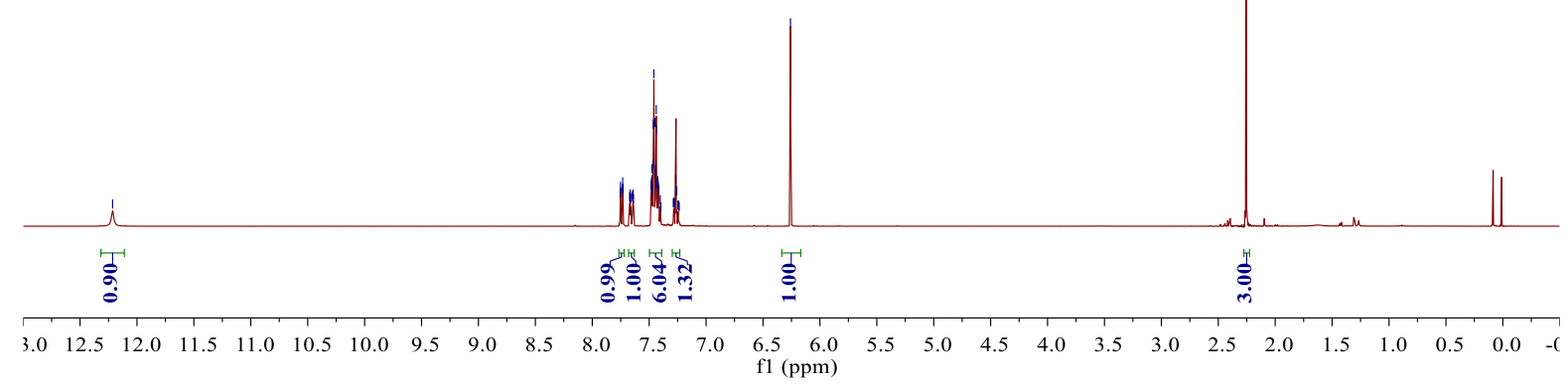

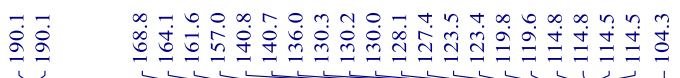

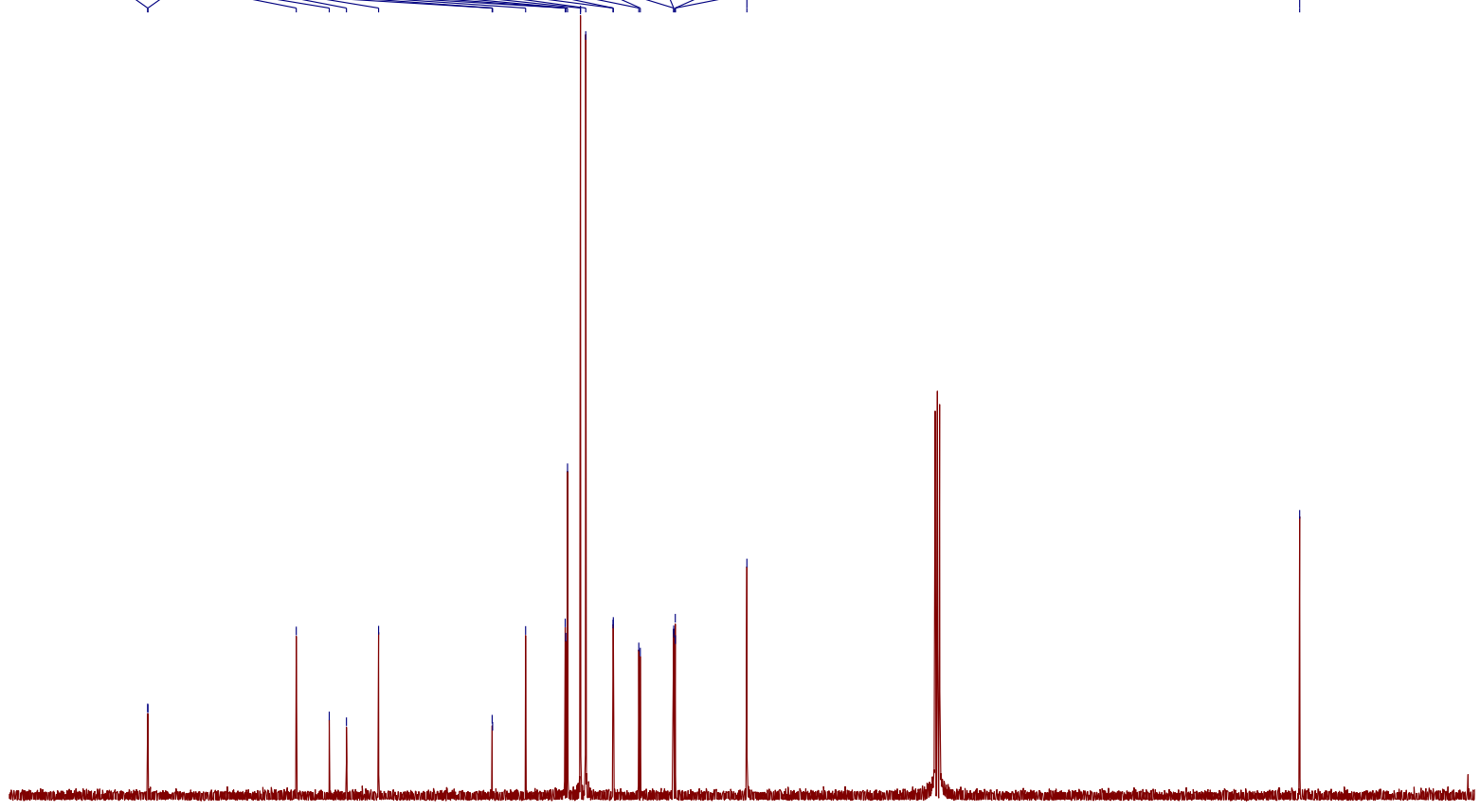

$\begin{array}{llllllllll}200 & 190 & 180 & 170 & 160 & 150 & 140 & 130 & 120 & 110 \quad \begin{array}{l}100 \\ \text { f1 (ppm) }\end{array}\end{array}$ 


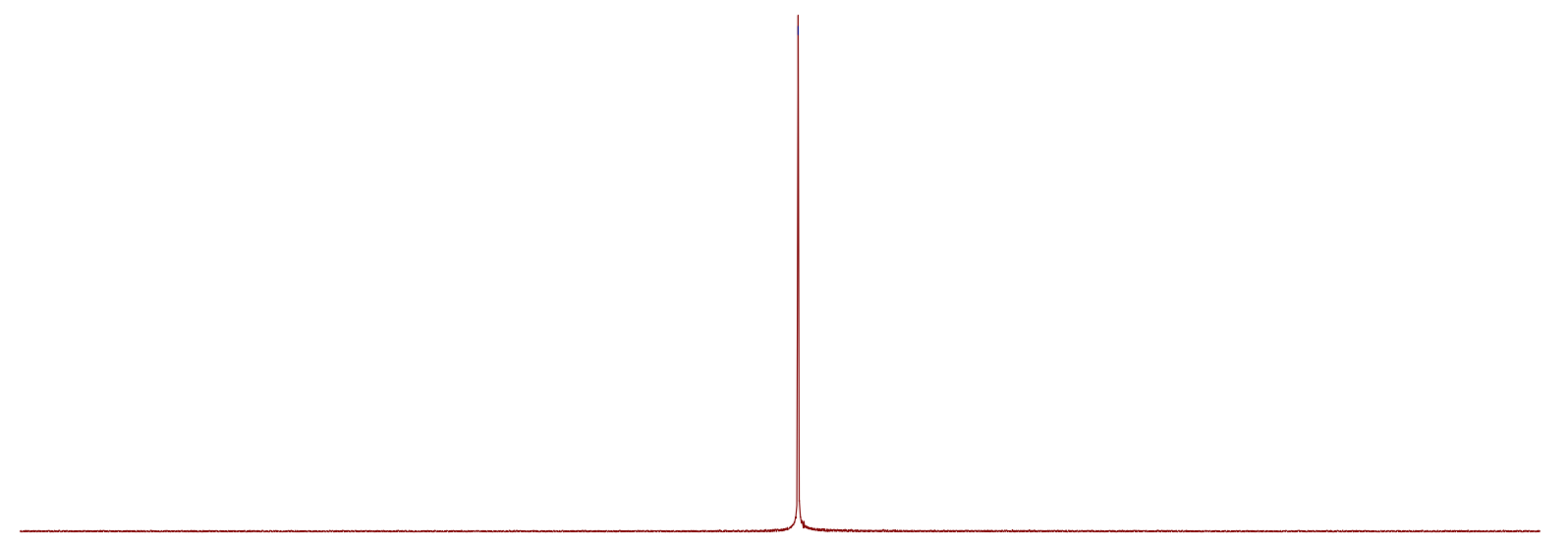

\begin{tabular}{lllllllllllllllllllllllllll}
\hline & 0 & -10 & -20 & -30 & -40 & -50 & -60 & -70 & -80 & -90 & -100 & -110 & -120 & -130 & -140 & -150 & -160 & -170 & -180 & -190 & -200 & -210
\end{tabular}

${ }^{1} \mathrm{H},{ }^{19} \mathrm{~F}$, and ${ }^{13} \mathrm{C}$ NMR spectra for product $3 \mathrm{~d}\left(\mathrm{CDCl}_{3}\right)$ 


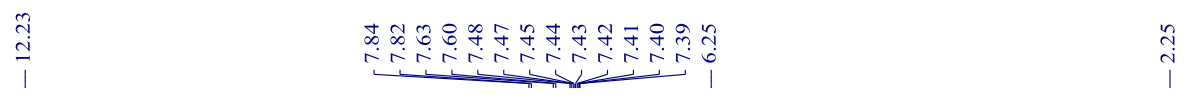<smiles>CC(=O)N/C(=C\C(=O)c1ccc(Br)cc1)c1ccccc1</smiles>

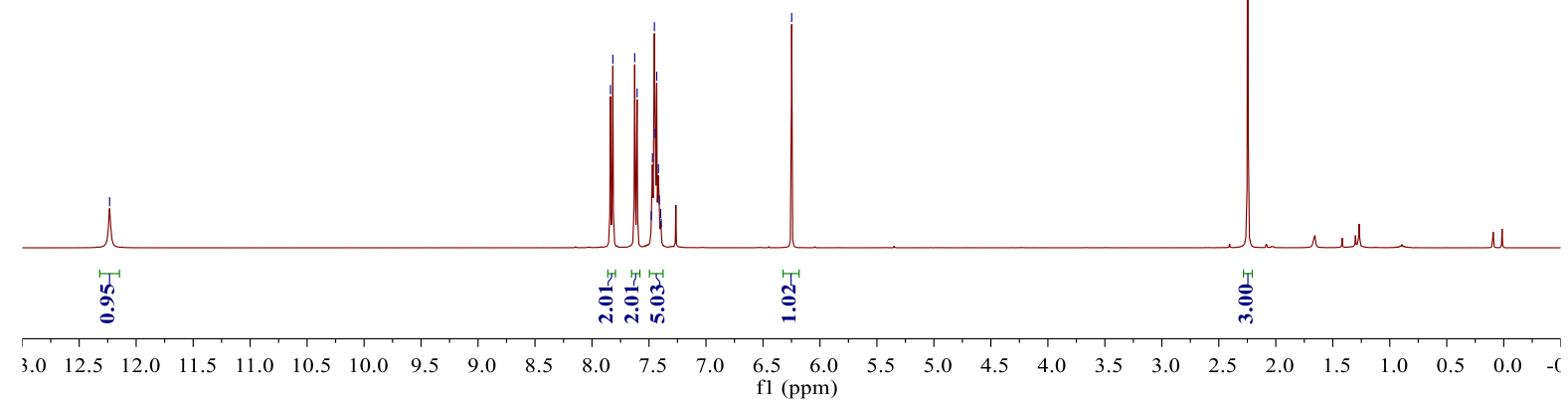

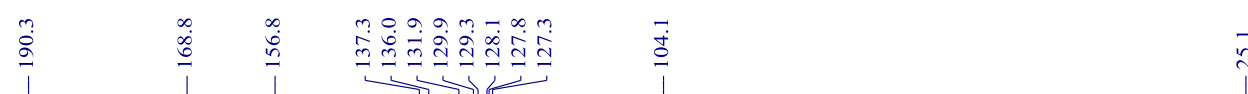

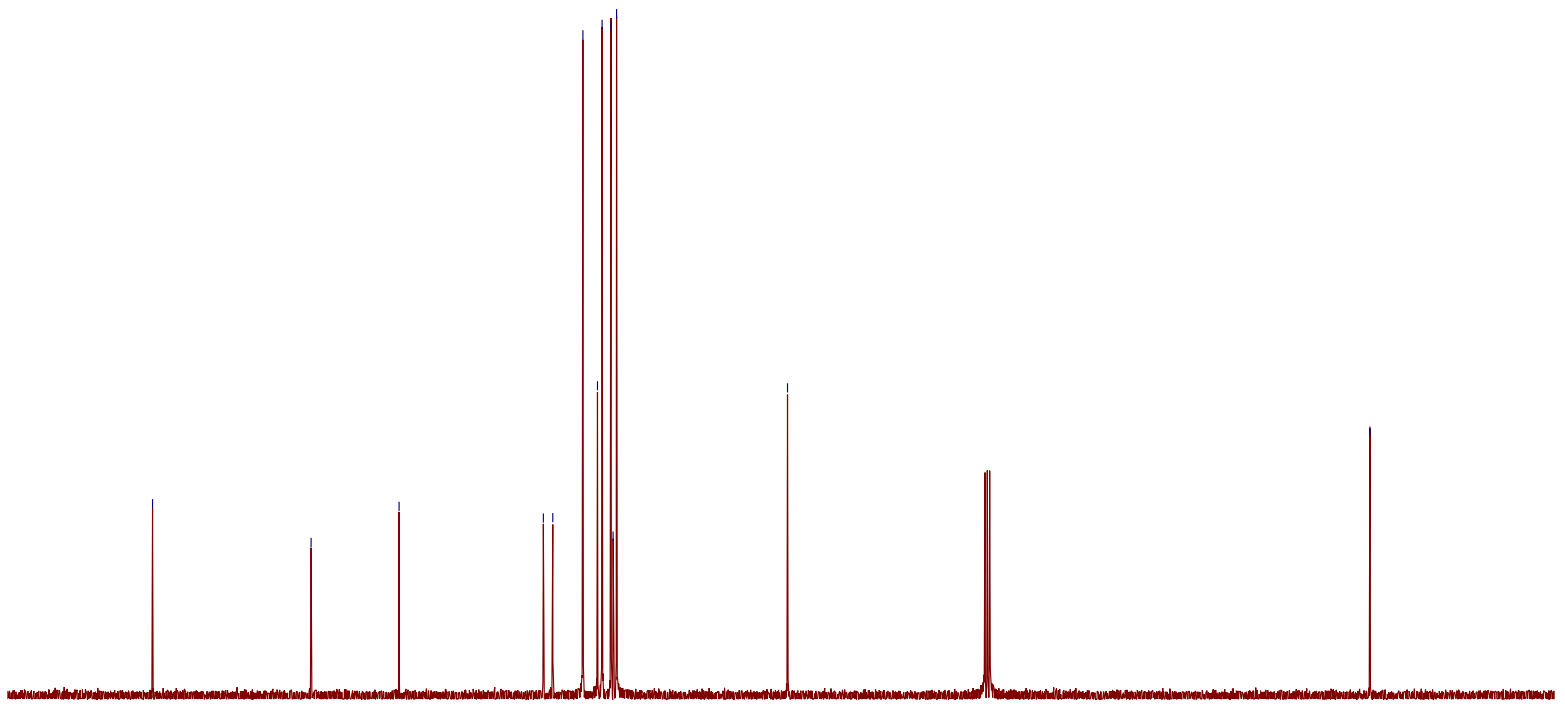

$\begin{array}{rrrrrrrrrrr}200 & 190 & 180 & 170 & 160 & 150 & 140 & 130 & 120 & 110 \begin{array}{r}100 \\ \text { fl (ppm) }\end{array}\end{array}$

${ }^{1} \mathrm{H}$ and ${ }^{13} \mathrm{C}$ NMR spectra for product $3 \mathrm{e}\left(\mathrm{CDCl}_{3}\right)$ 


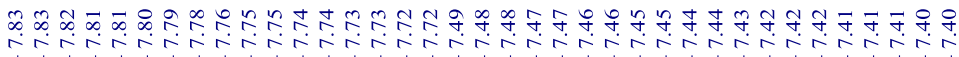<smiles>CC(=O)N/C(=C\C(=O)c1ccc(F)c(F)c1)c1ccccc1</smiles>

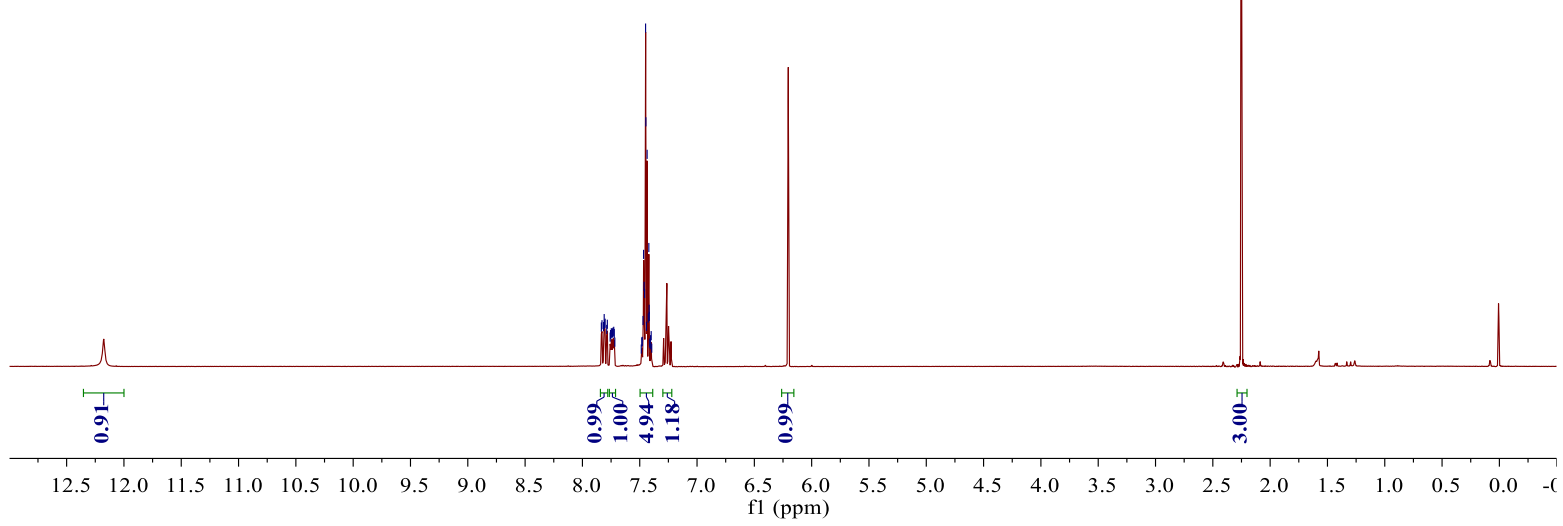

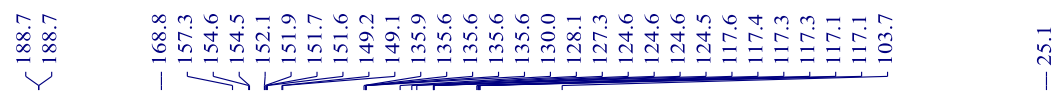

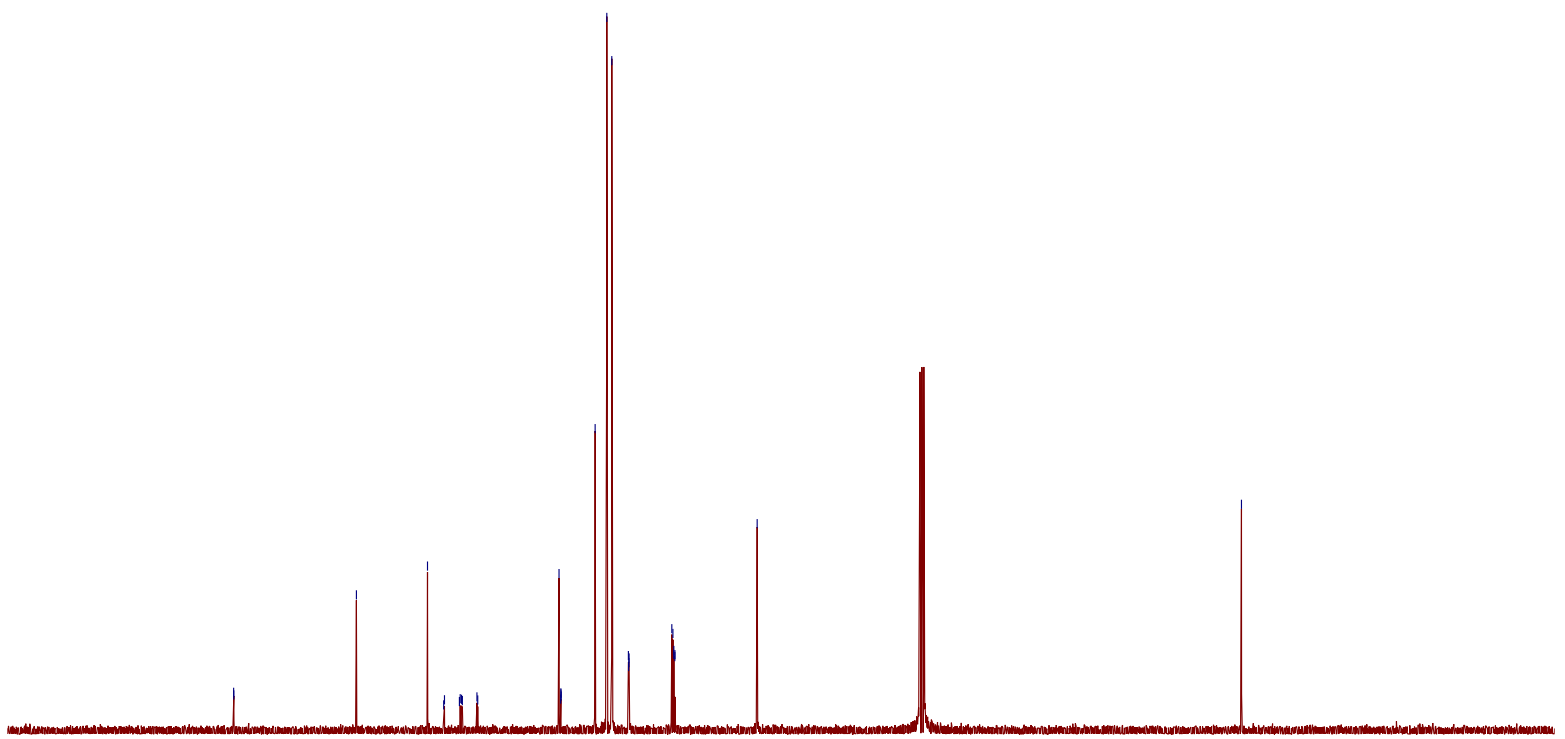

$\begin{array}{lllllllllllllllllllllllllllll}220 & 210 & 200 & 190 & 180 & 170 & 160 & 150 & 140 & 130 & 120 & 110 & 100 & 90 & 80 & 70 & 60 & 50 & 40 & 30 & 20 & 10 & 0 & -10 & -20\end{array}$ 


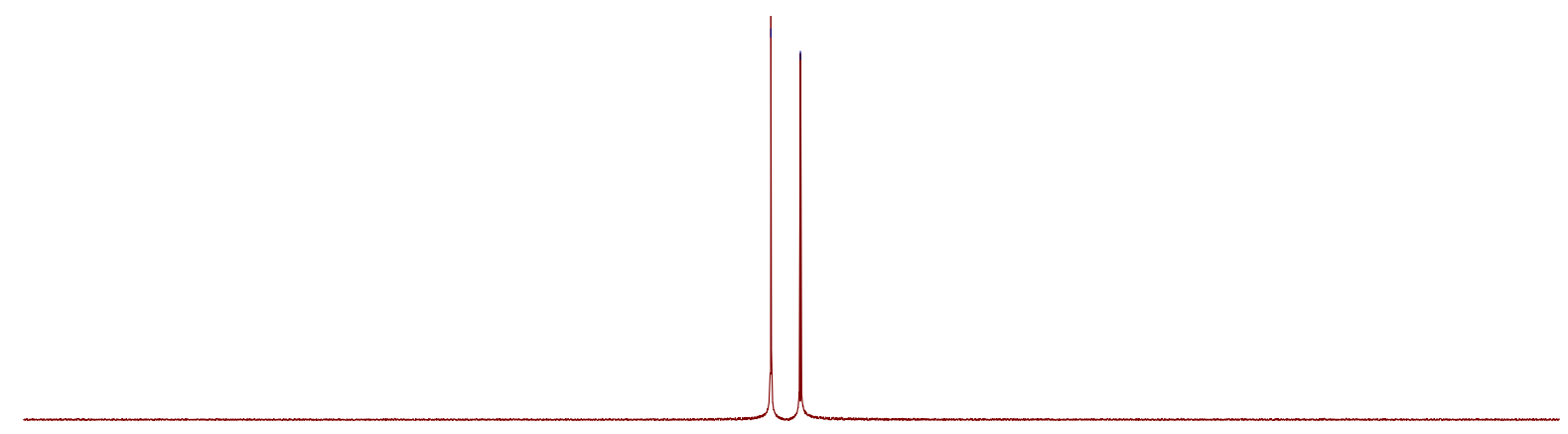

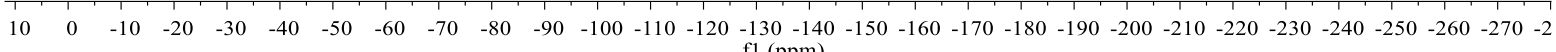
f1 (ppm)

${ }^{1} \mathrm{H},{ }^{19} \mathrm{~F}$, and ${ }^{13} \mathrm{C}$ NMR spectra for product $3 \mathrm{f}\left(\mathrm{CDCl}_{3}\right)$ 


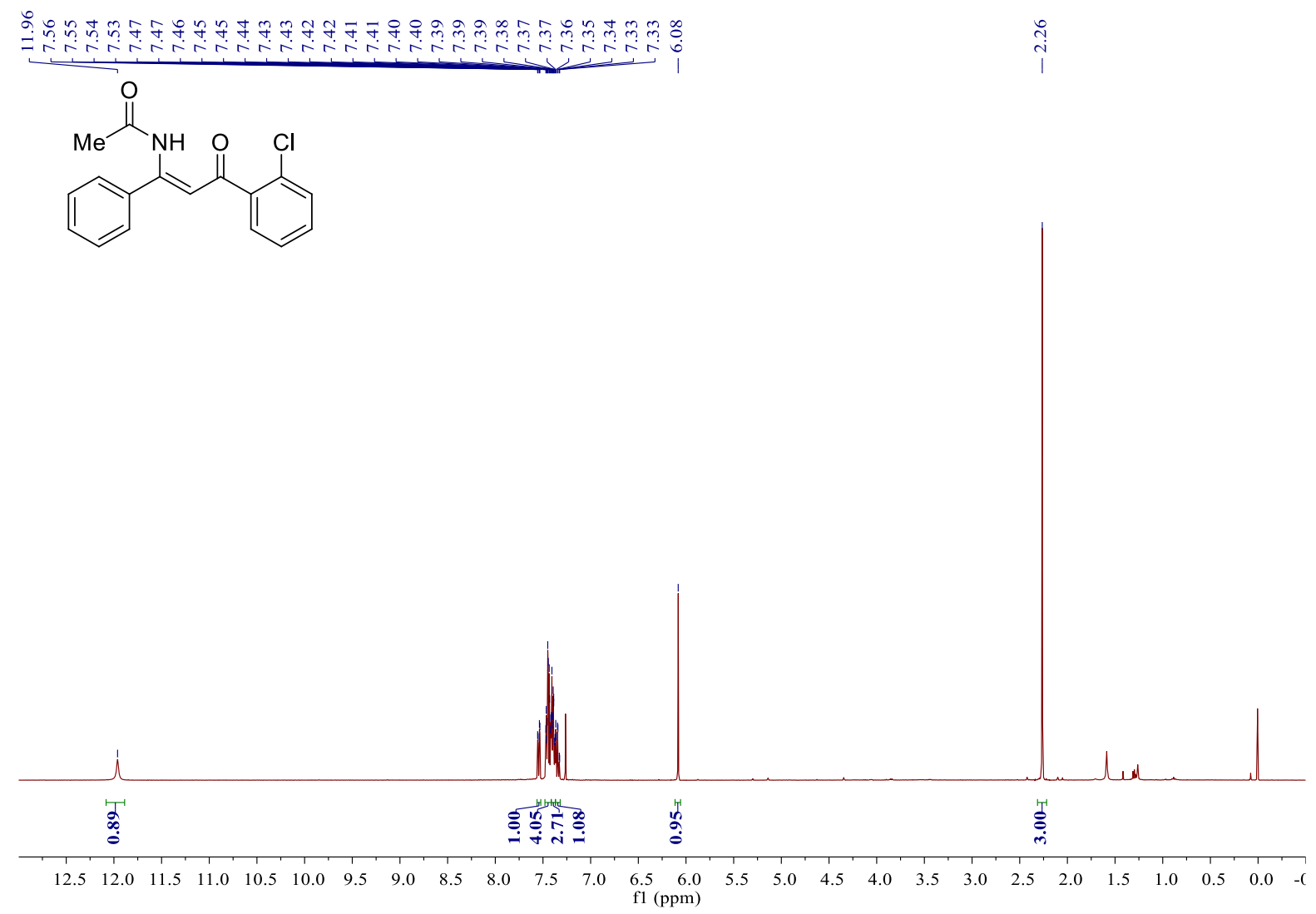

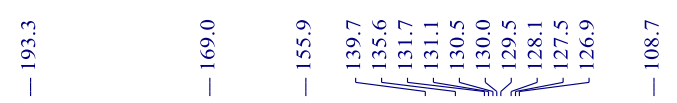

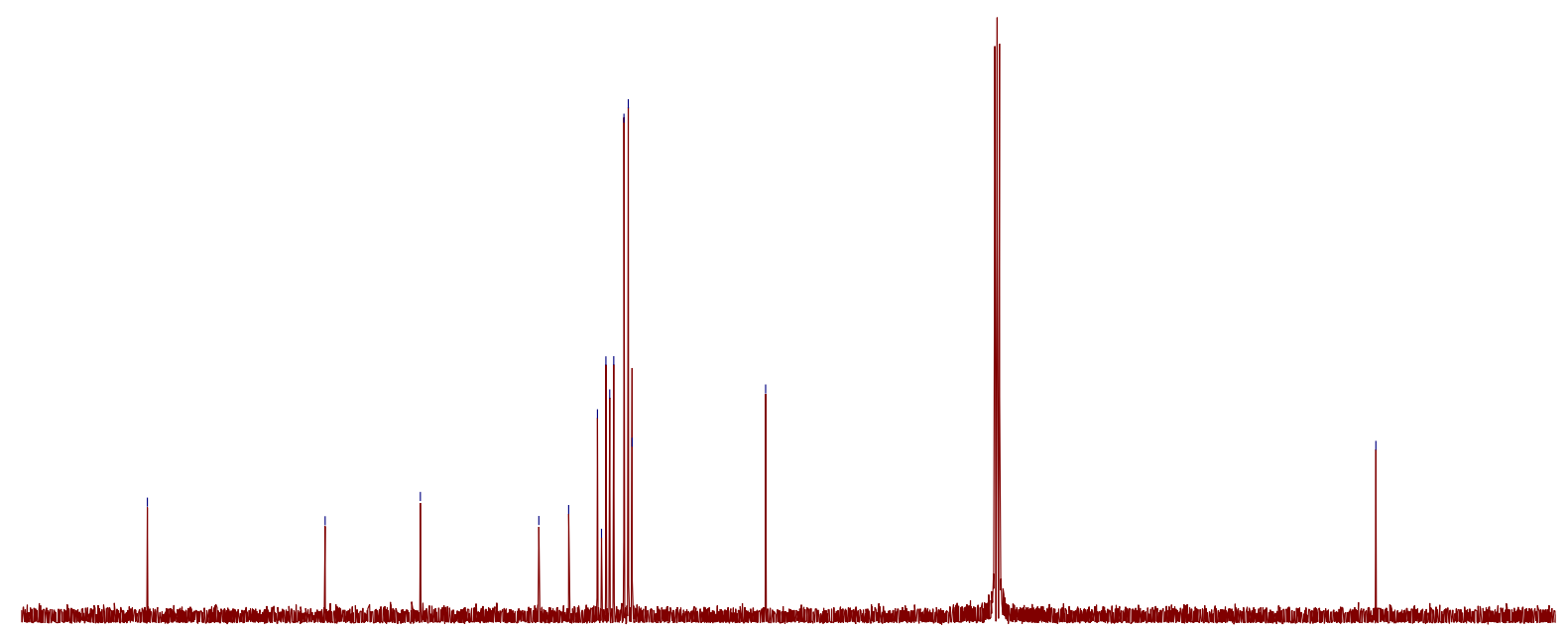

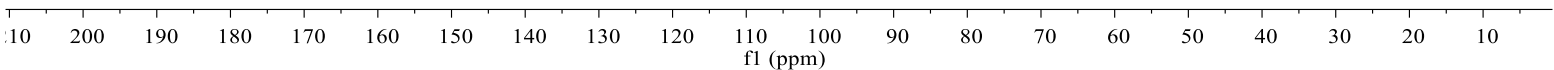

${ }^{1} \mathrm{H}$ and ${ }^{13} \mathrm{C}$ NMR spectra for product $3 \mathrm{~g}\left(\mathrm{CDCl}_{3}\right)$ 
<smiles>CC(=O)N/C(=C\C(=O)c1ccc(C#N)cc1)c1ccccc1</smiles>
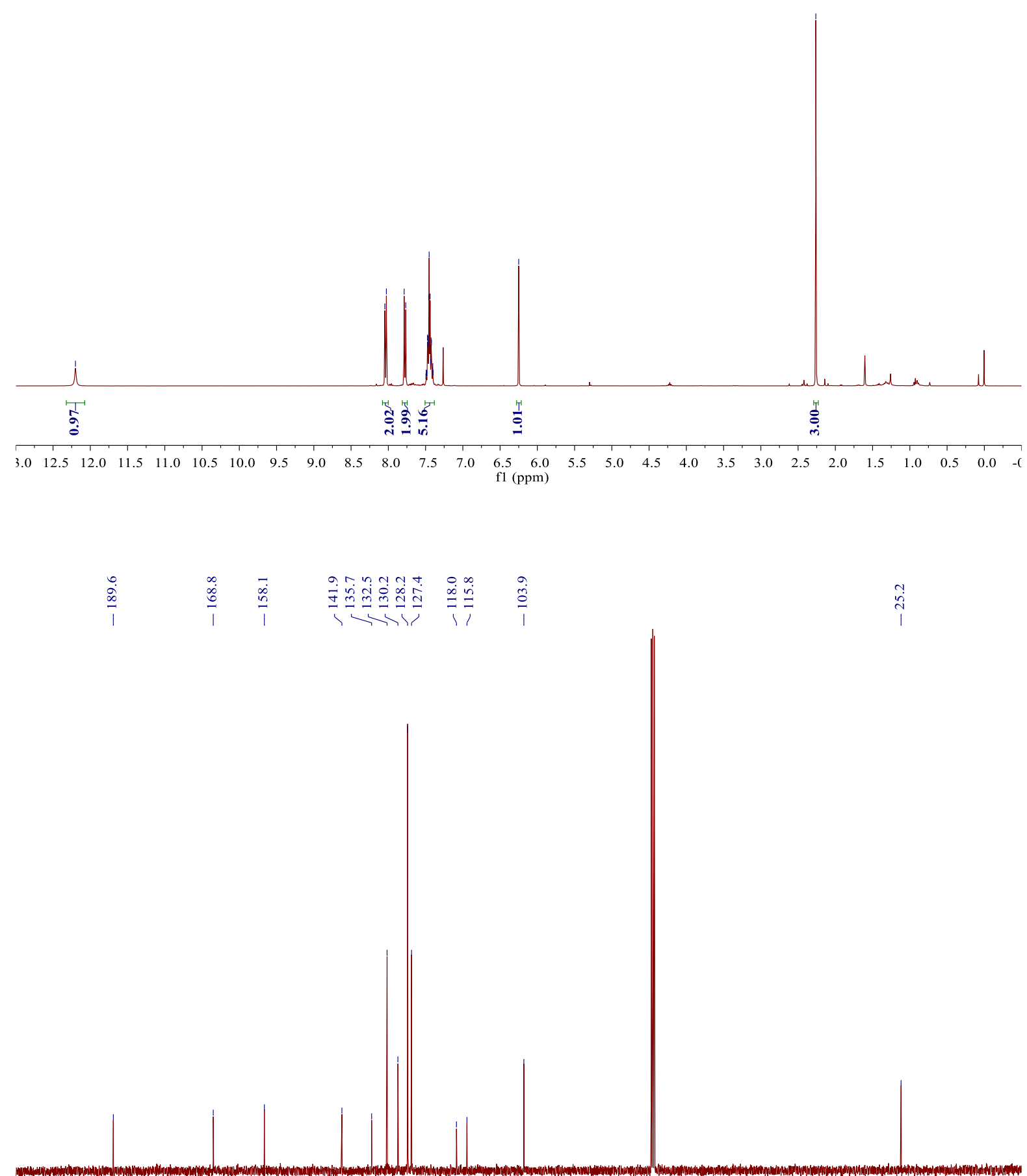

$\begin{array}{lllllllllll}10 & 200 & 190 & 180 & 170 & 160 & 150 & 140 & 130 & 120 & \begin{array}{c}110 \\ \text { f1 (ppm) }\end{array}\end{array}$

${ }^{1} \mathrm{H}$ and ${ }^{13} \mathrm{C}$ NMR spectra for product $3 \mathrm{~h}\left(\mathrm{CDCl}_{3}\right)$ 
$\underbrace{0}$<smiles>CC(=O)N/C(=C\C(=O)c1ccc(C(F)(F)F)cc1)c1ccccc1</smiles>

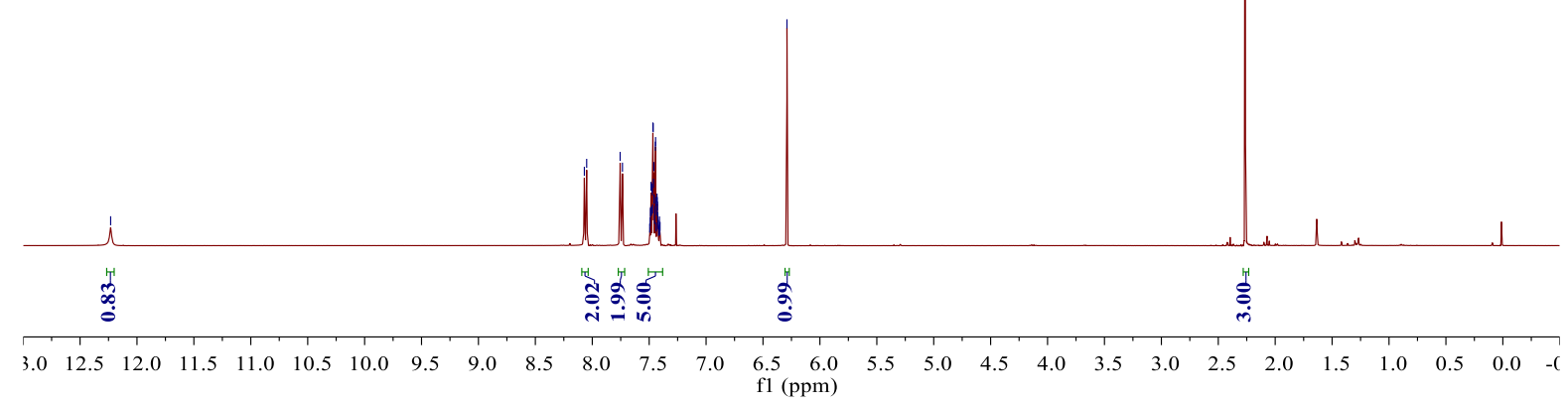

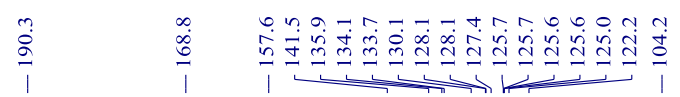

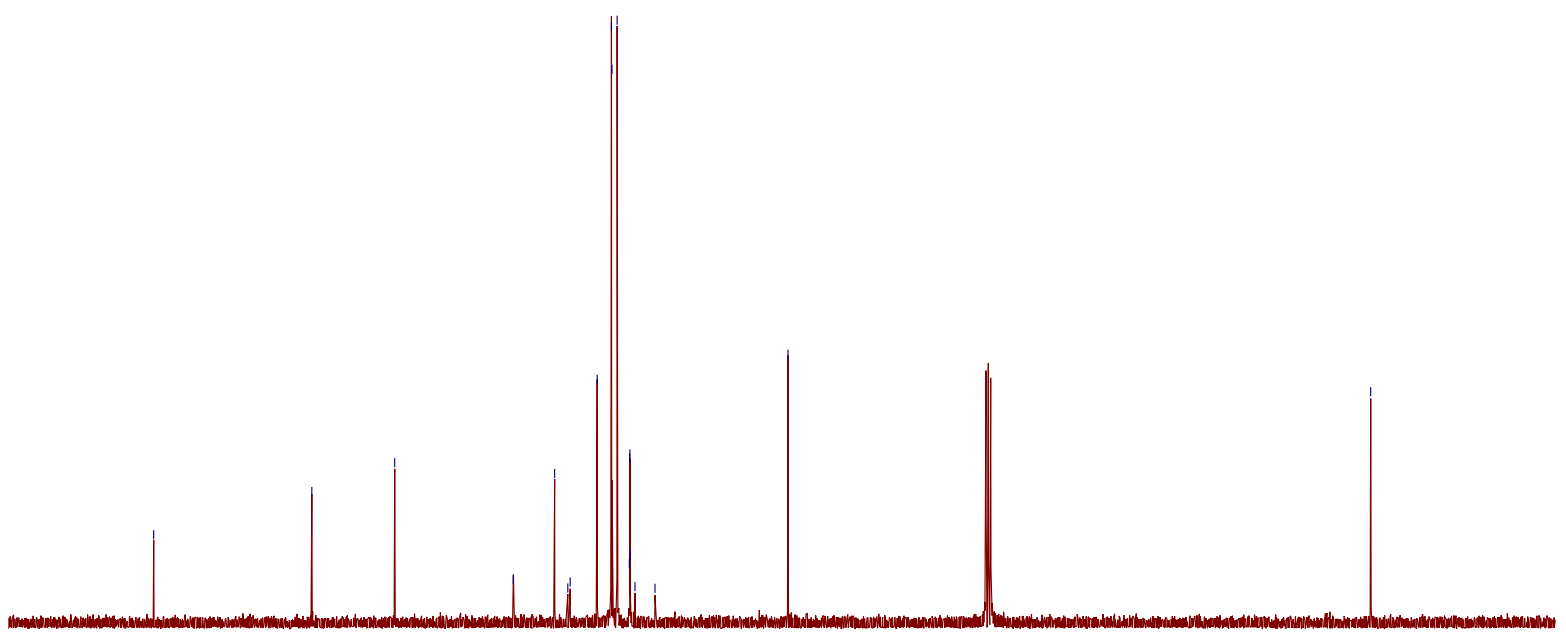

$\begin{array}{llllllllll}200 & 190 & 180 & 170 & 160 & 150 & 140 & 130 & 120 & \begin{array}{c}110 \\ \text { fl (ppm) }\end{array}\end{array}$ 


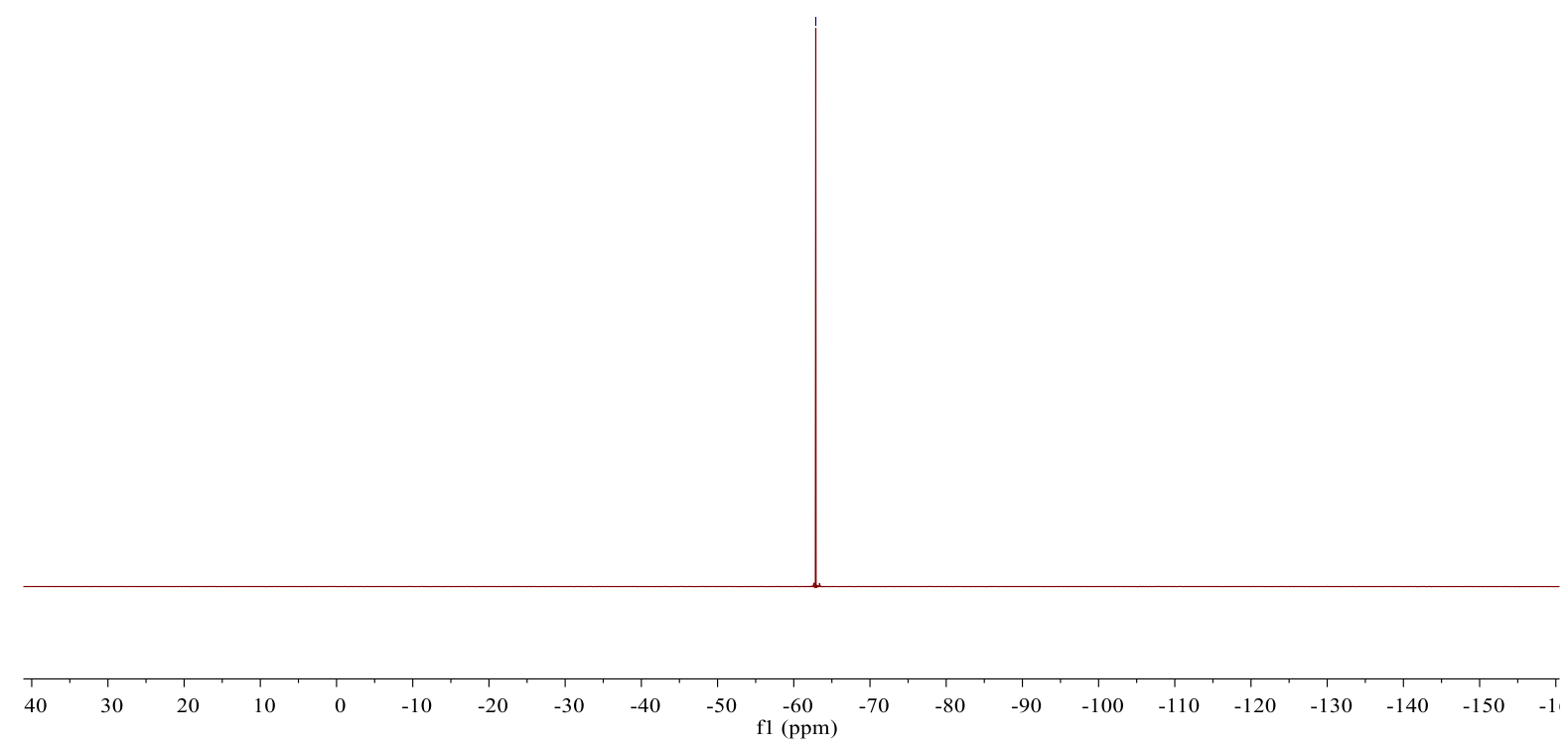

${ }^{1} \mathrm{H},{ }^{19} \mathrm{~F}$, and ${ }^{13} \mathrm{C}$ NMR spectra for product $3 \mathrm{i}(\mathrm{CDCl})$ 

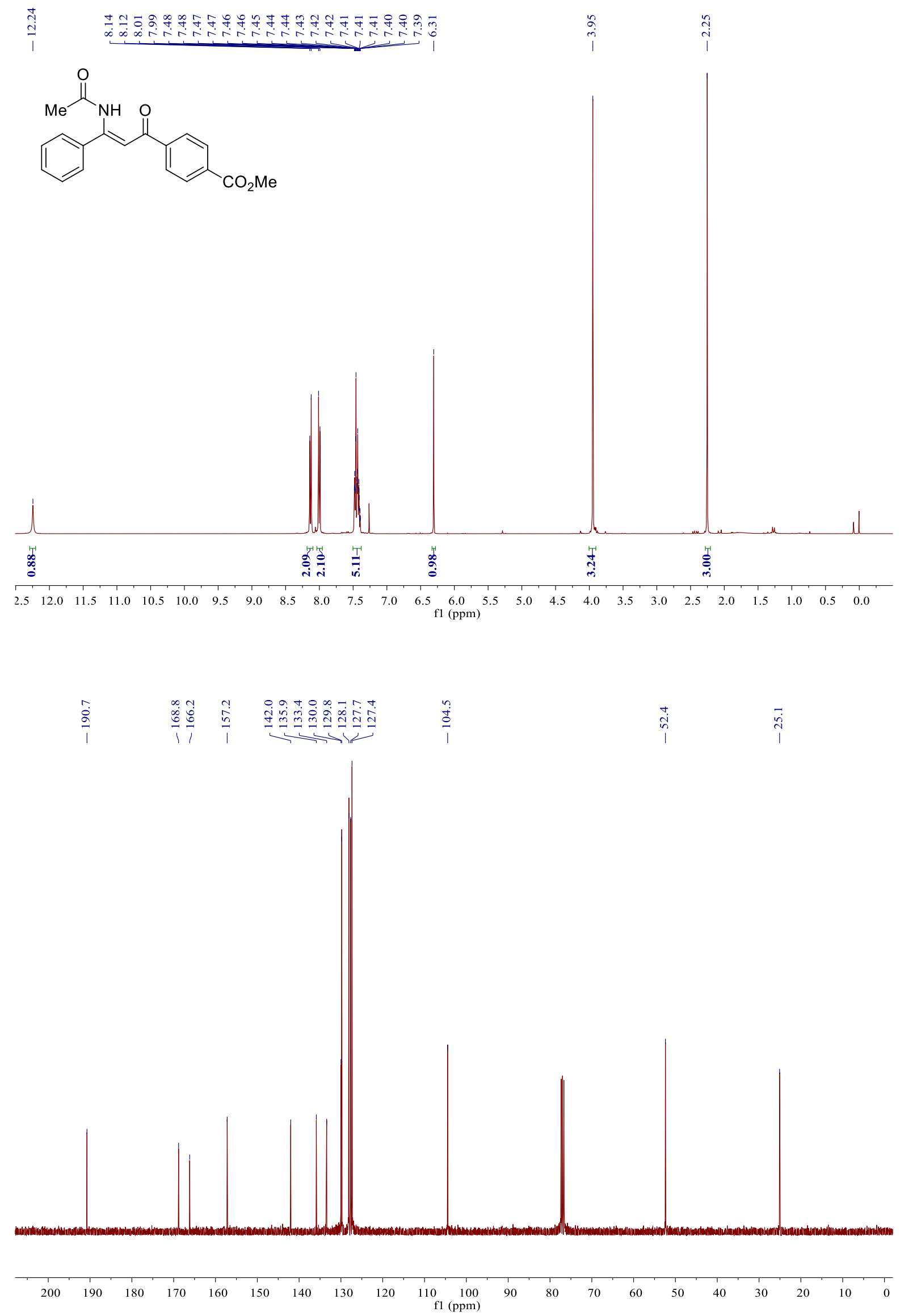

${ }^{1} \mathrm{H}$ and ${ }^{13} \mathrm{C}$ NMR spectra for product $3 \mathrm{j}\left(\mathrm{CDCl}_{3}\right)$ 

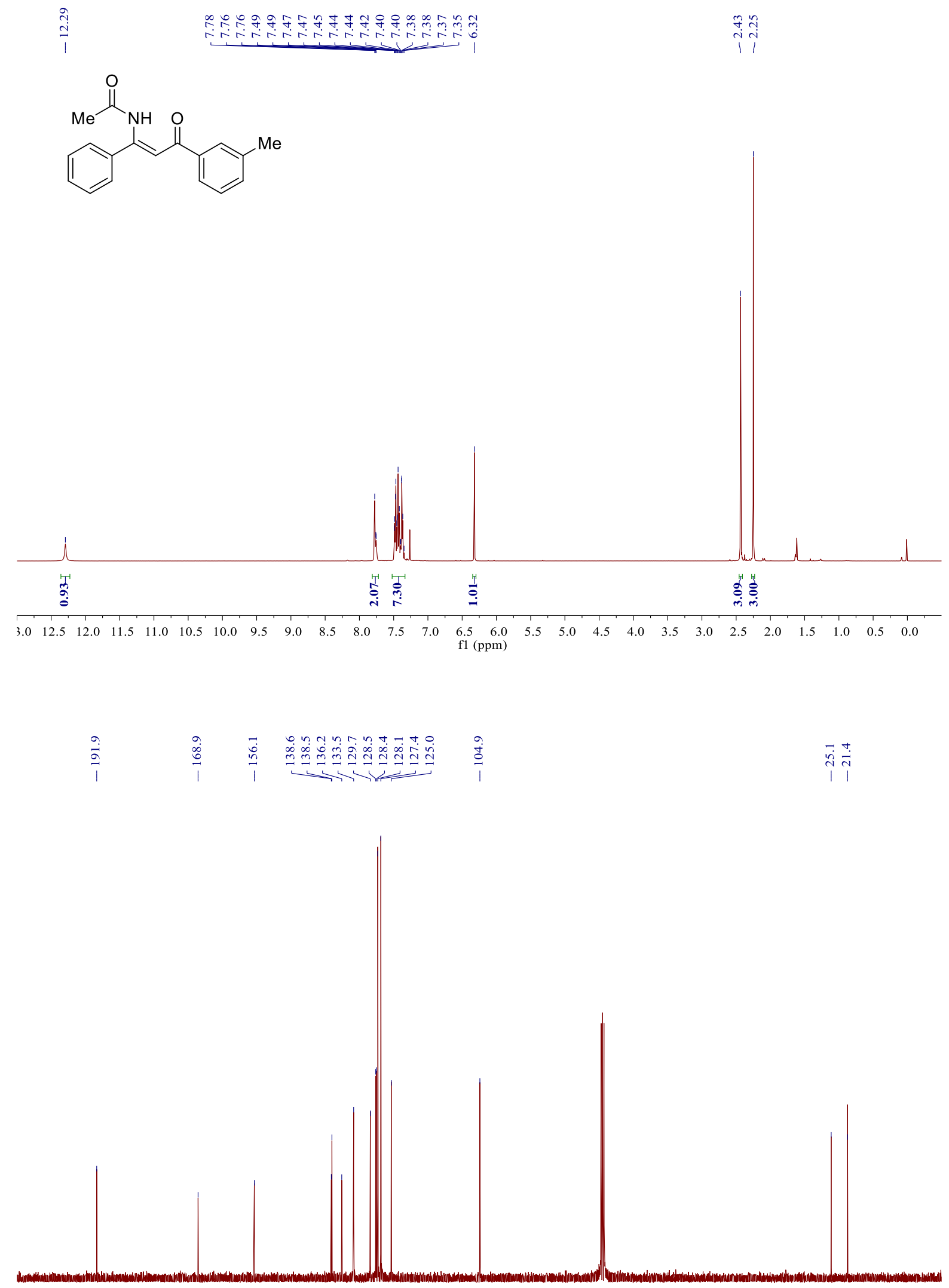

$\begin{array}{llllllllll}200 & 190 & 180 & 170 & 160 & 150 & 140 & 130 & 120 & \begin{array}{c}110 \\ \text { f1 (ppm) }\end{array}\end{array}$

${ }^{1} \mathrm{H}$ and ${ }^{13} \mathrm{C}$ NMR spectra for product $3 \mathrm{k}\left(\mathrm{CDCl}_{3}\right)$ 


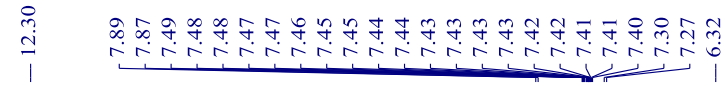<smiles>CC(=O)N/C(=C\C(=O)c1ccc(C)cc1)c1ccccc1</smiles>
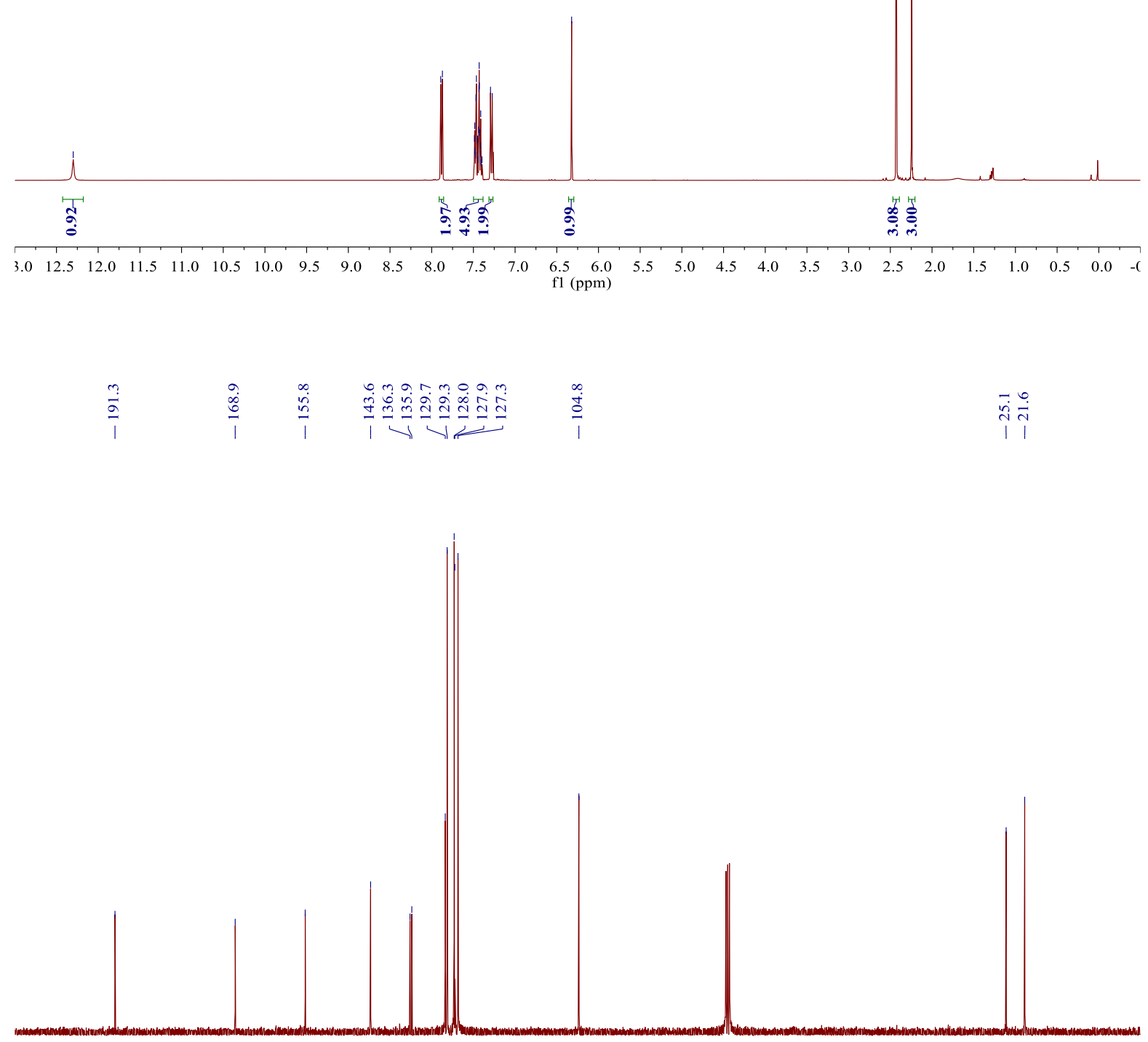

$\begin{array}{lllllllllllllllllll}200 & 190 & 180 & 170 & 160 & 150 & 140 & 130 & 120 & \begin{array}{c}110 \\ \mathrm{fl}(\mathrm{pmm})\end{array} & 90 & 80 & 70 & 60 & 50 & 40 & 30 & 20 & 10\end{array}$

${ }^{1} \mathrm{H}$ and ${ }^{13} \mathrm{C}$ NMR spectra for product $3 \mathrm{I}\left(\mathrm{CDCl}_{3}\right)$ 

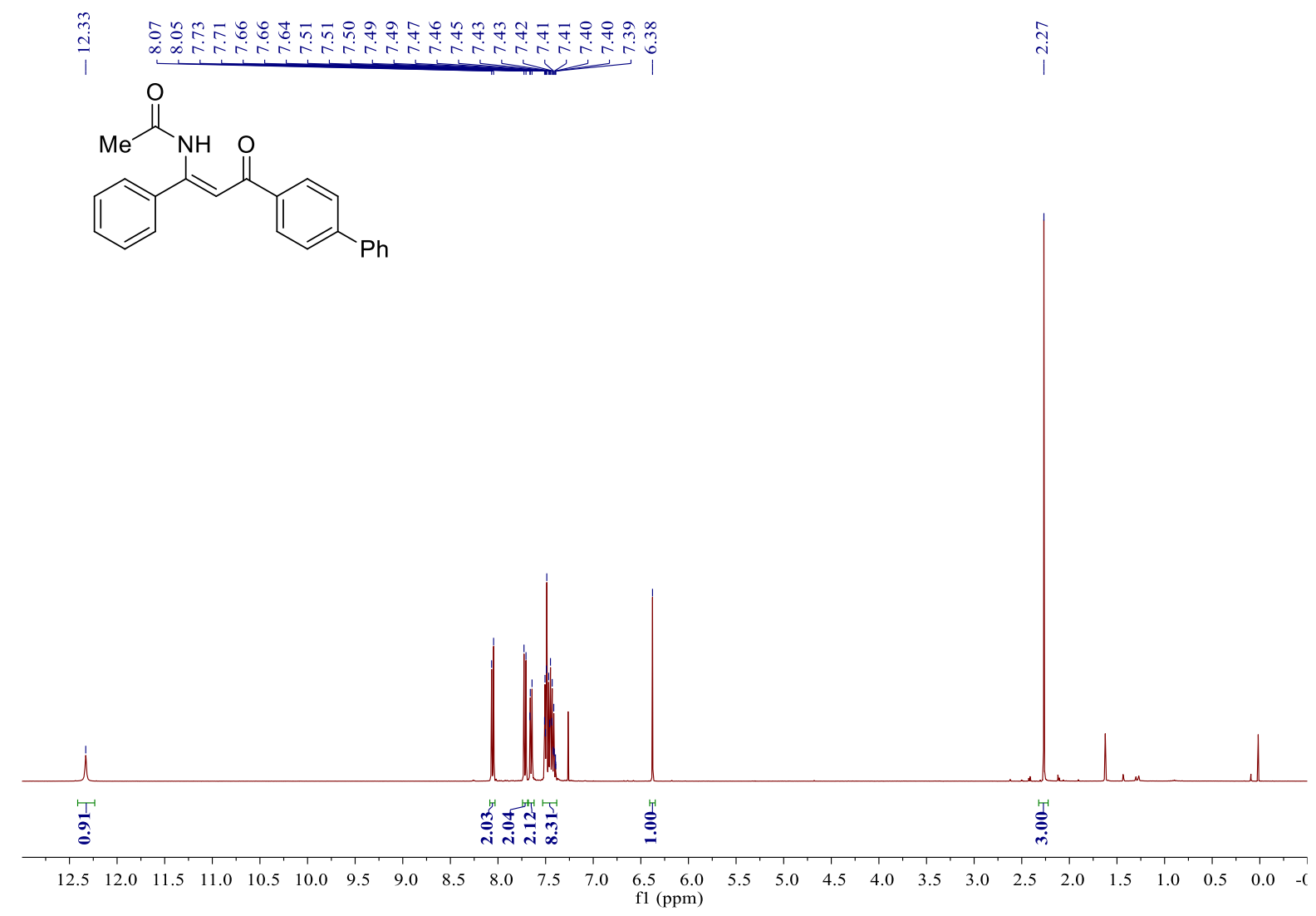

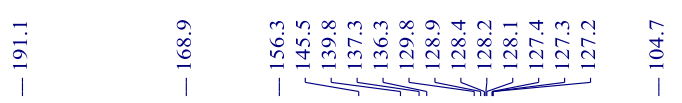

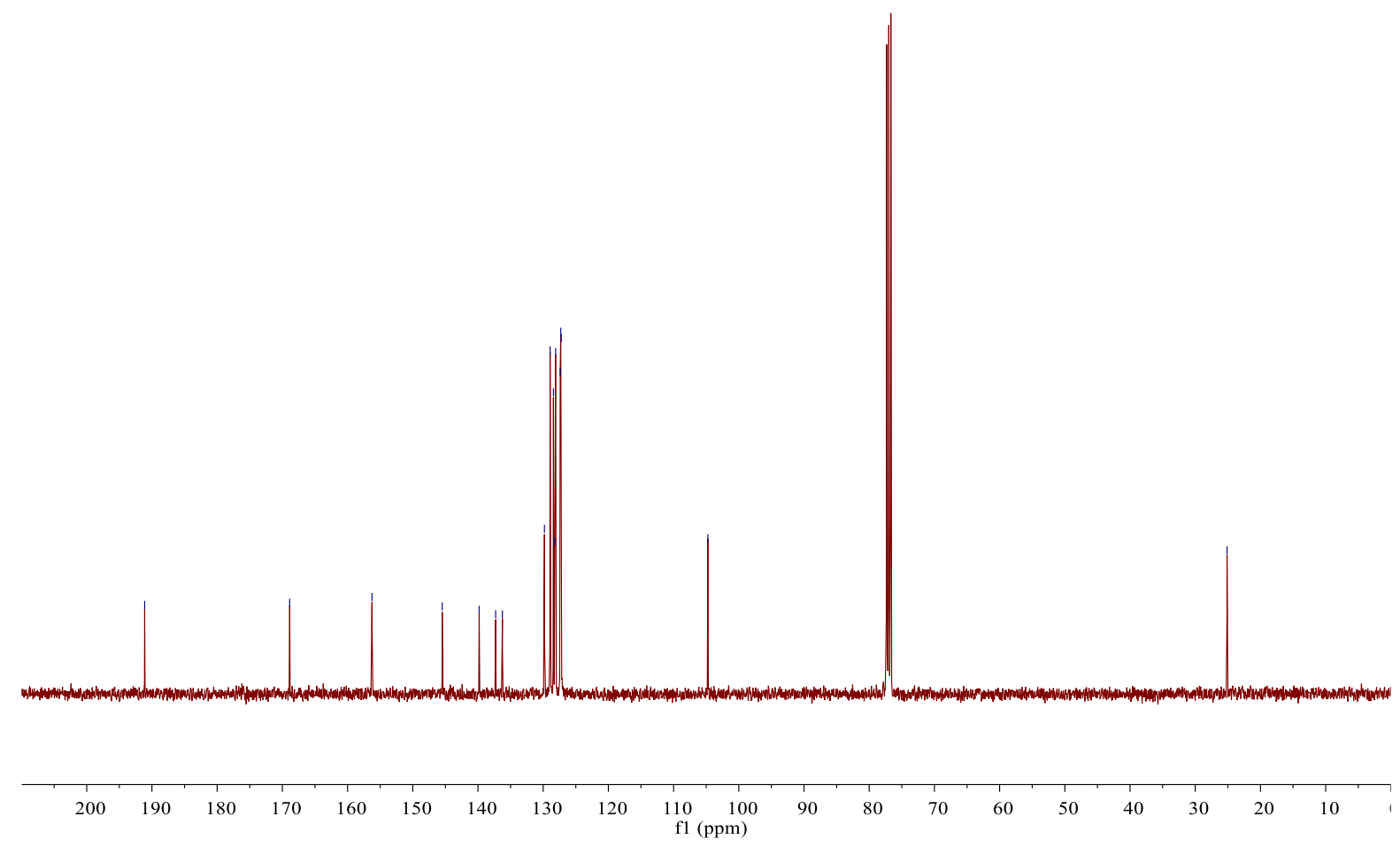

${ }^{1} \mathrm{H}$ and ${ }^{13} \mathrm{C}$ NMR spectra for product $3 \mathrm{~m}\left(\mathrm{CDCl}_{3}\right)$ 


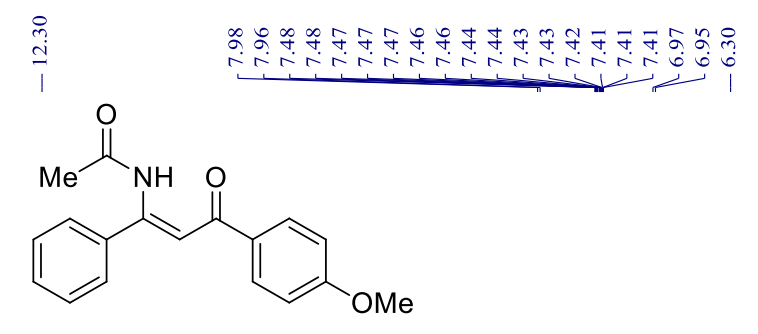

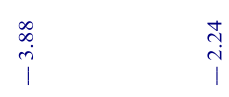

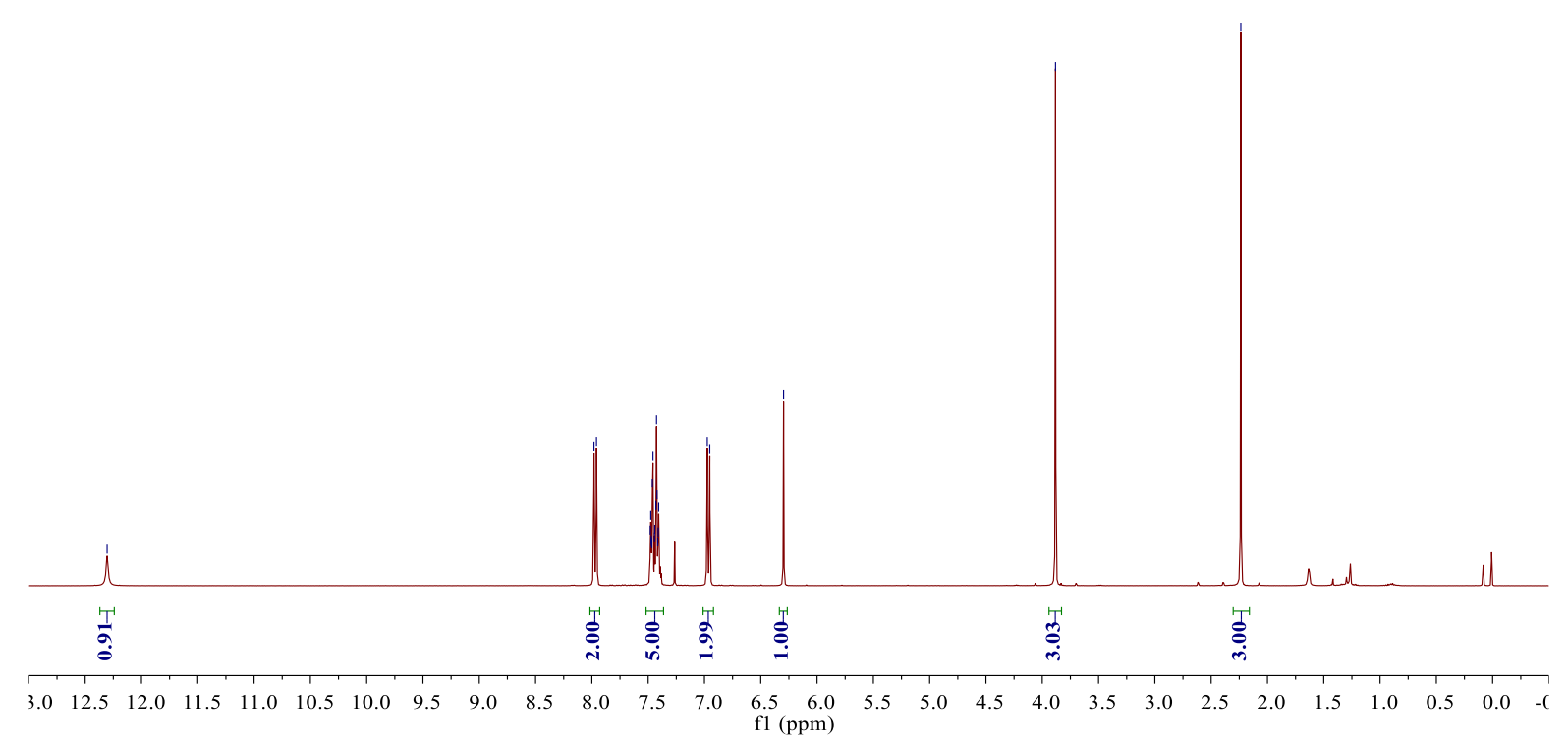

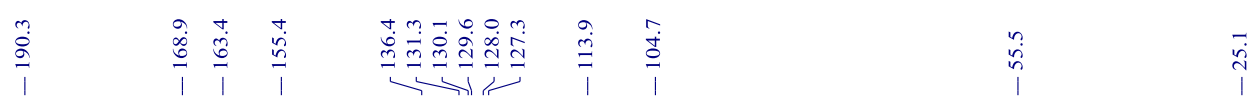

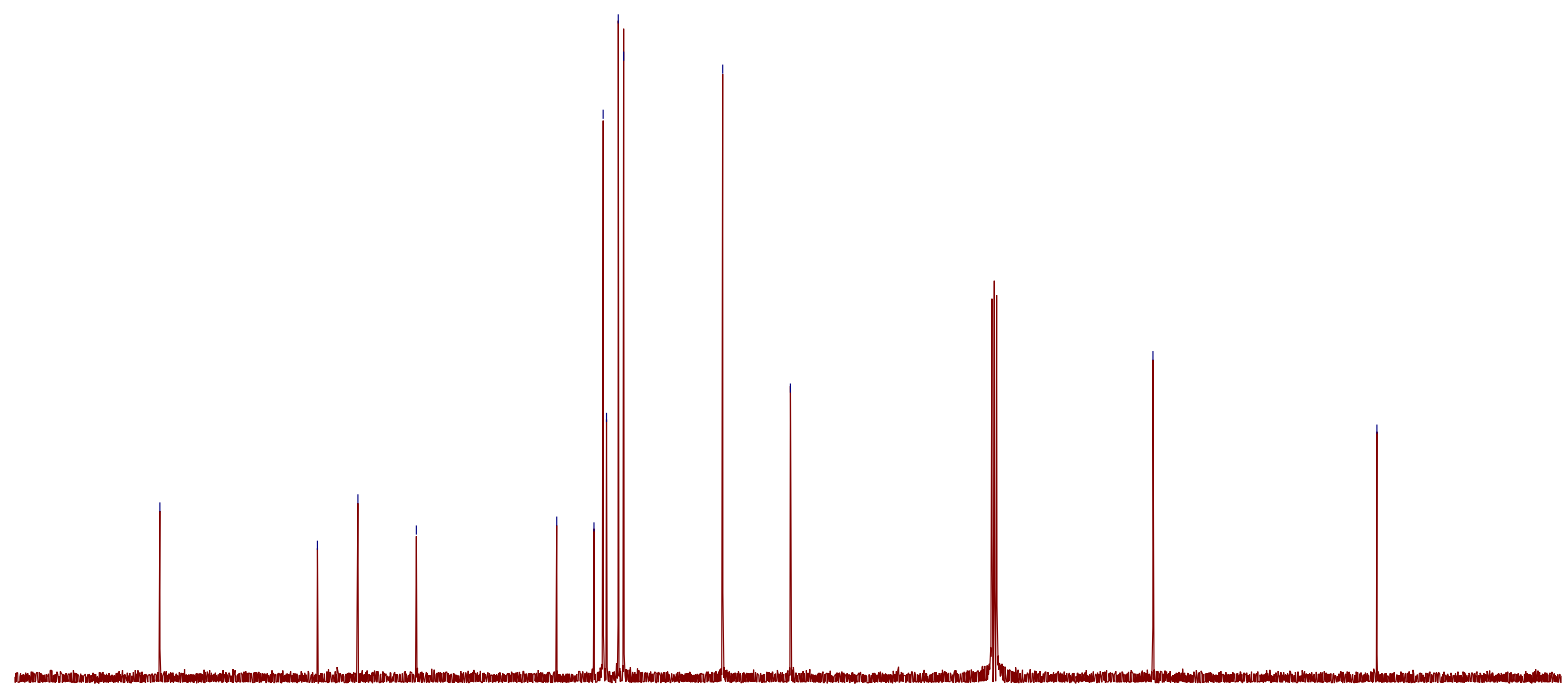

$\begin{array}{llllllllllllllllllllll}200 & 190 & 180 & 170 & 160 & 150 & 140 & 130 & 120 & \begin{array}{c}110 \\ \mathrm{f} 1(\mathrm{ppm})\end{array} & 90 & 80 & 70 & 60 & 50 & 40 & 30 & 20 & 10 & 10\end{array}$

${ }^{1} \mathrm{H}$ and ${ }^{13} \mathrm{C}$ NMR spectra for product $3 \mathrm{n}\left(\mathrm{CDCl}_{3}\right)$ 


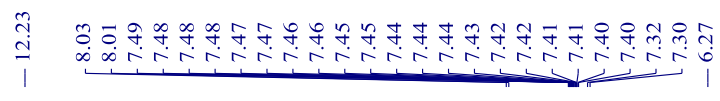<smiles>CC(=O)N/C(=C\C(=O)c1ccc(OC(F)(F)F)cc1)c1ccccc1</smiles>

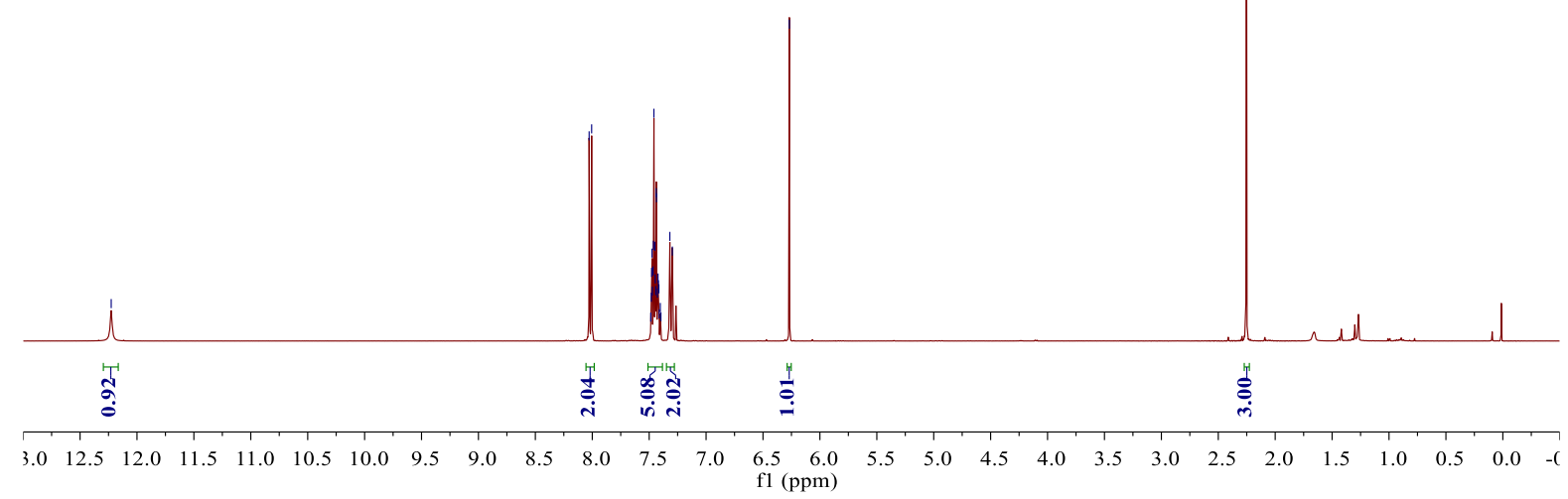

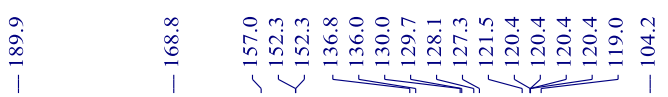

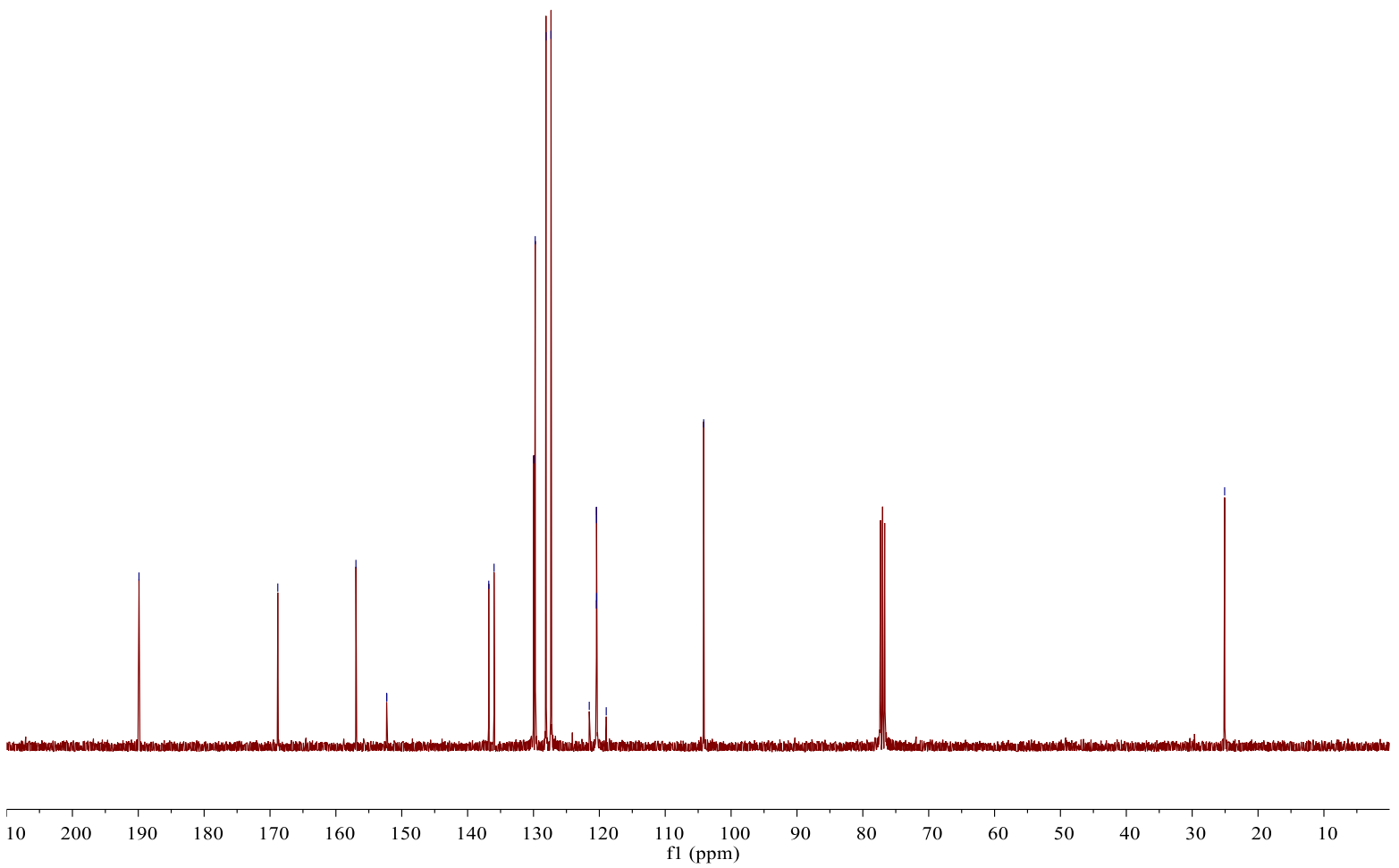




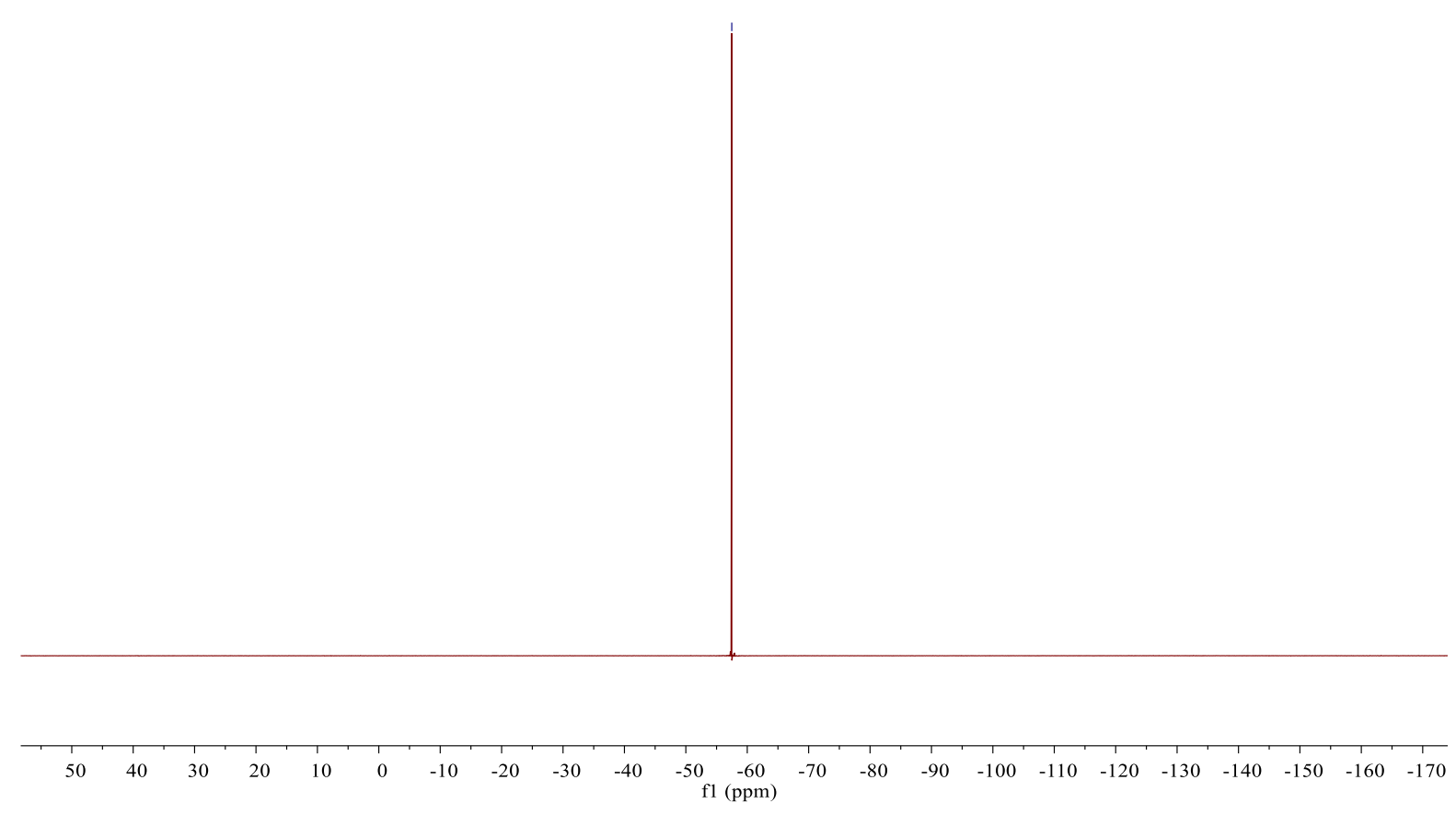

${ }^{1} \mathrm{H},{ }^{19} \mathrm{~F}$, and ${ }^{13} \mathrm{C}$ NMR spectra for product $30\left(\mathrm{CDCl}_{3}\right)$ 

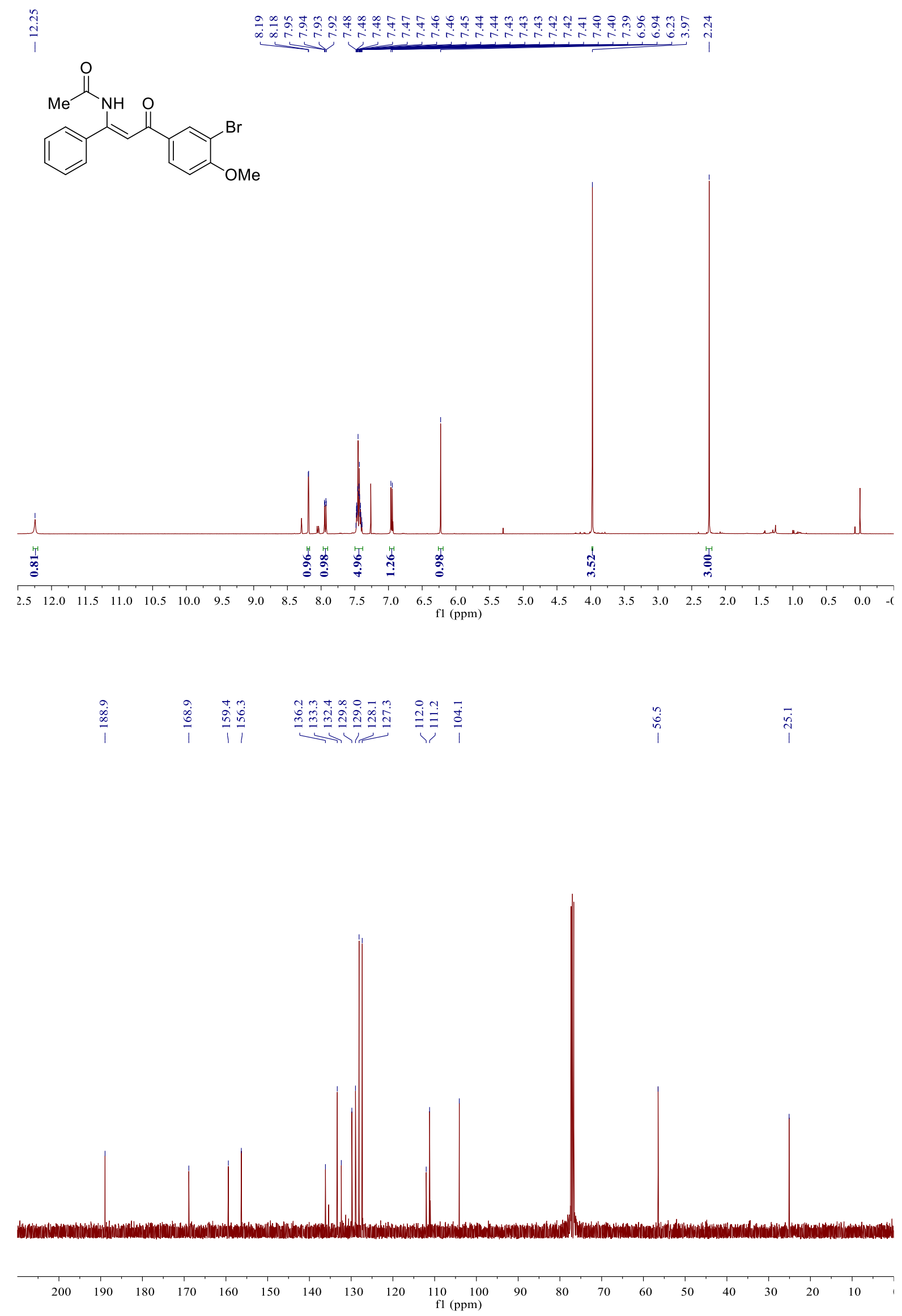

${ }^{1} \mathrm{H}$ and ${ }^{13} \mathrm{C}$ NMR spectra for product $3 \mathrm{p}\left(\mathrm{CDCl}_{3}\right)$ 


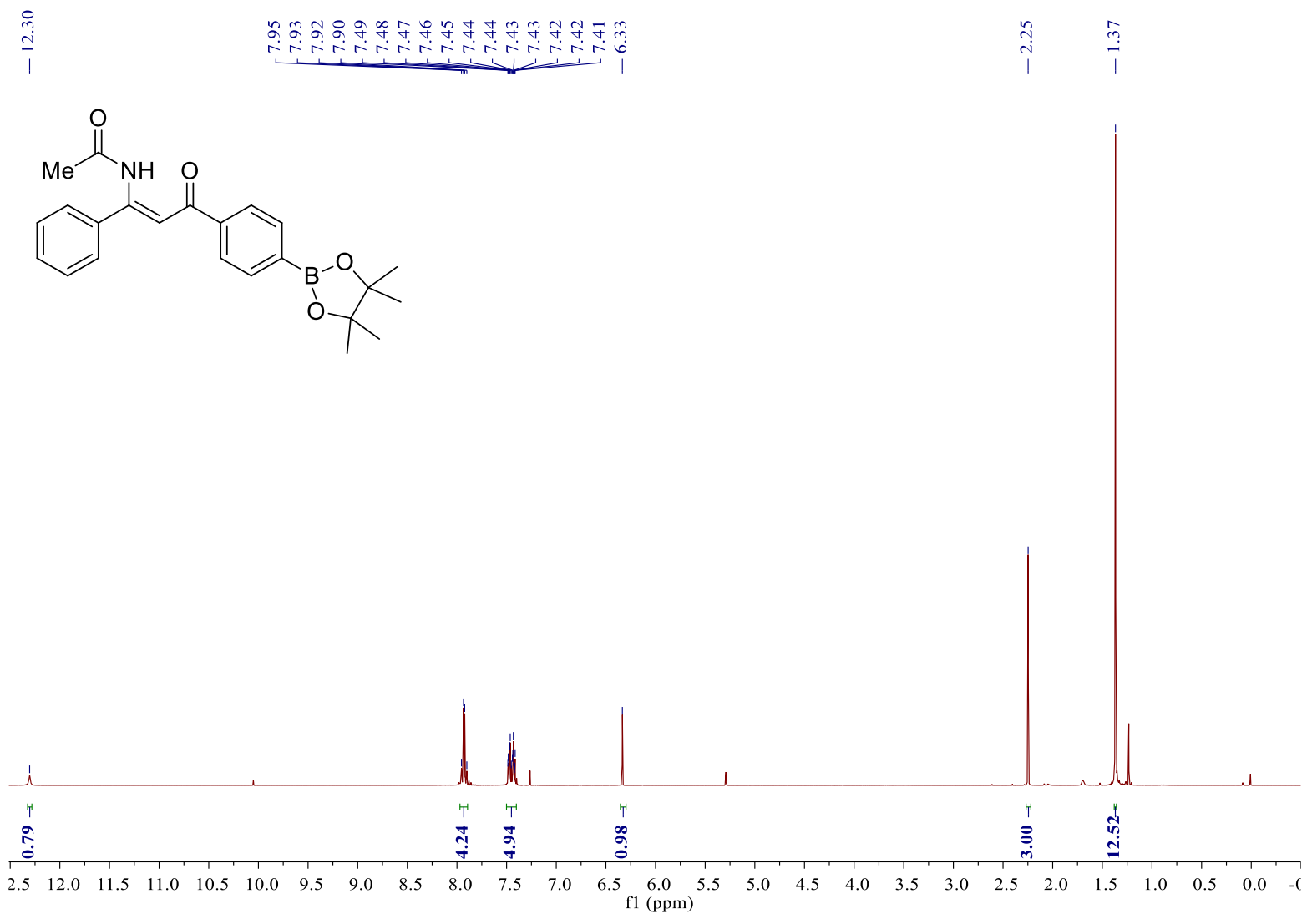

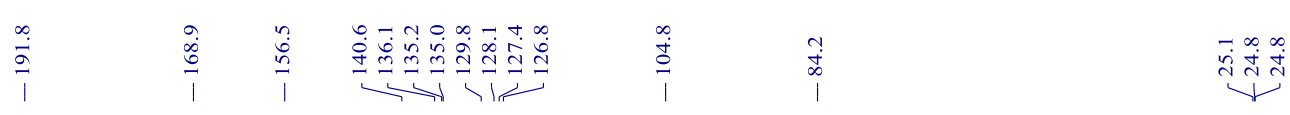

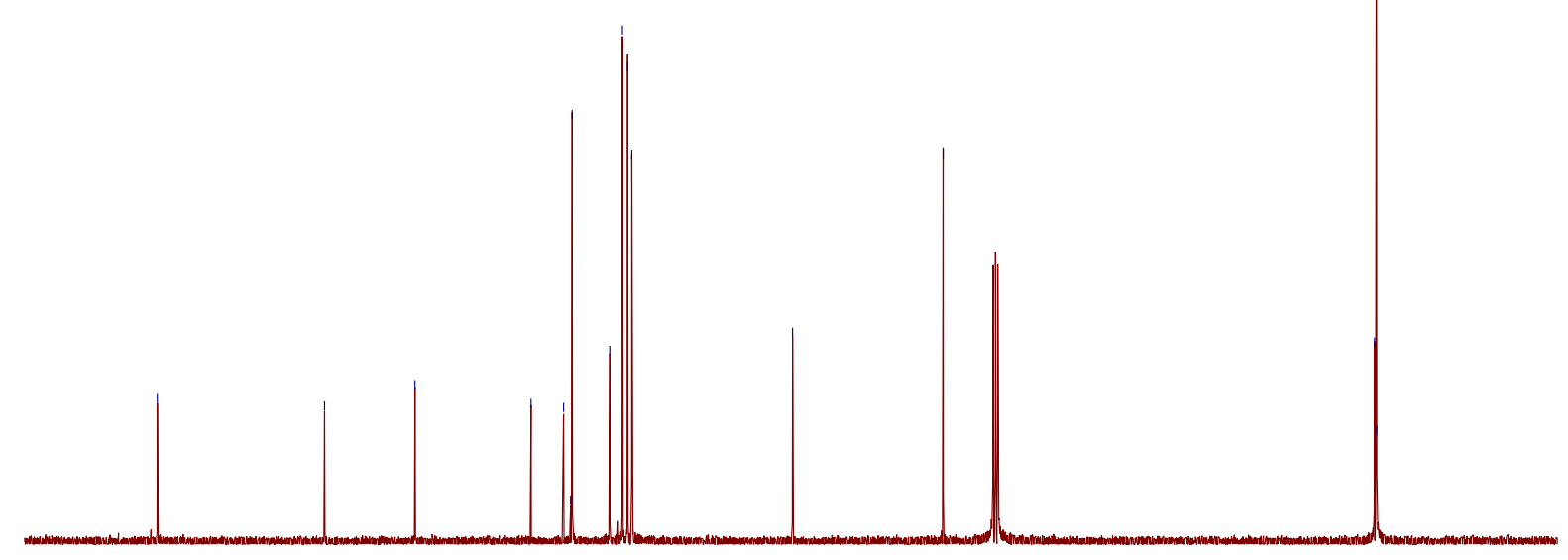

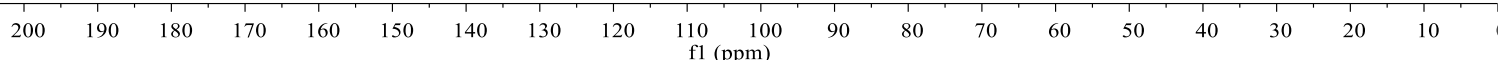

${ }^{1} \mathrm{H}$ and ${ }^{13} \mathrm{C}$ NMR spectra for product $3 \mathrm{q}\left(\mathrm{CDCl}_{3}\right)$ 
<smiles>CC(=O)N/C(=C\C(=O)c1ccc(C#CC(C)(C)C)cc1)c1ccccc1</smiles>
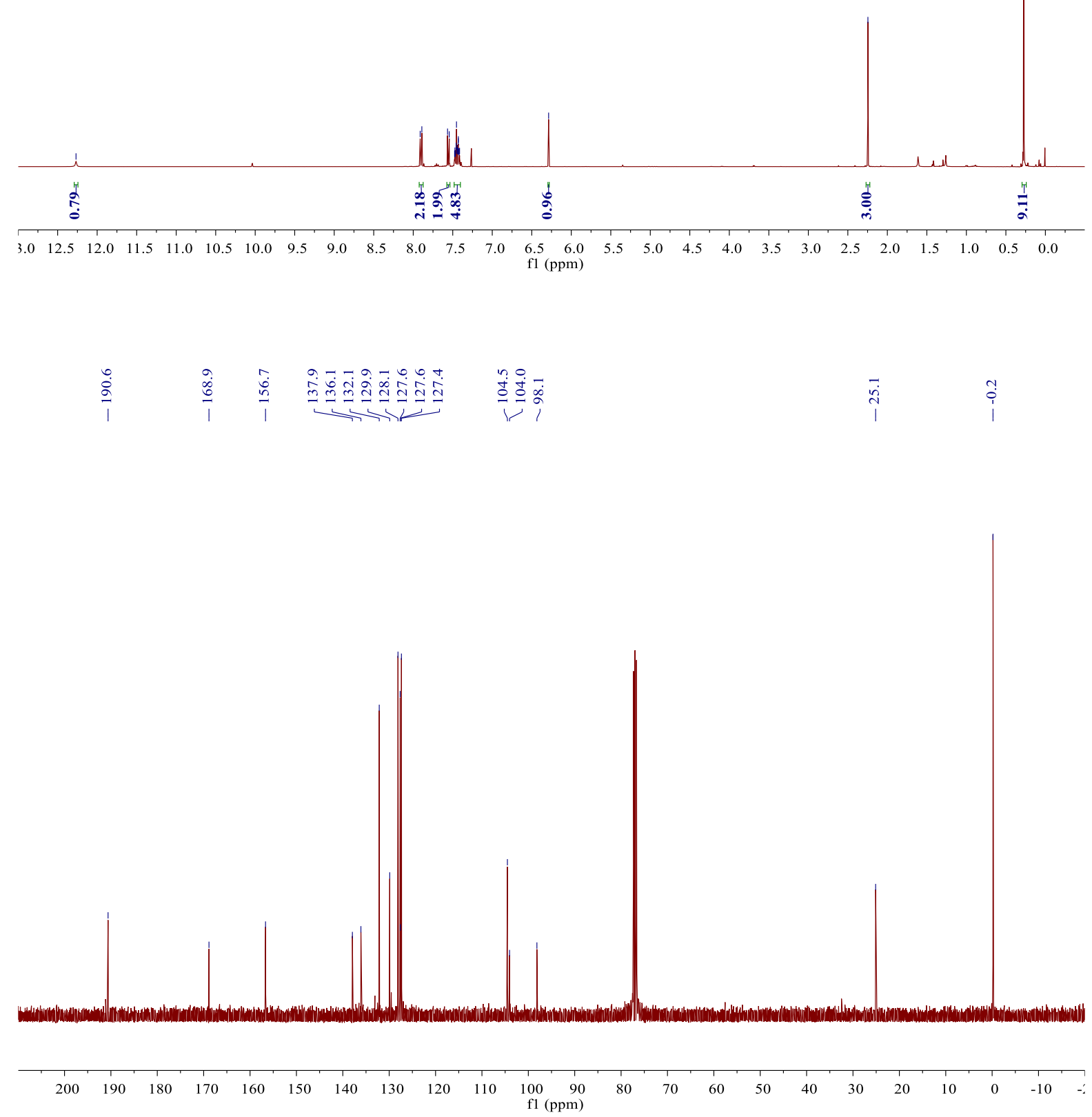

${ }^{1} \mathrm{H}$ and ${ }^{13} \mathrm{C}$ NMR spectra for product $3 \mathrm{r}\left(\mathrm{CDCl}_{3}\right)$ 
<smiles>CC(=O)N/C(=C\C(=O)c1ccc2ccccc2c1)c1ccccc1</smiles>

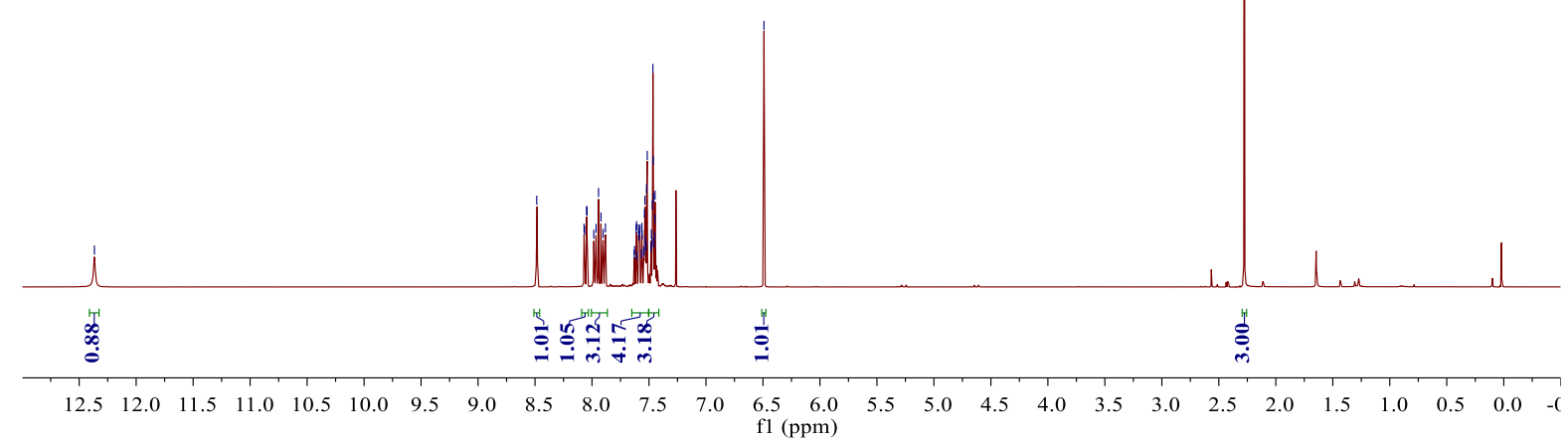

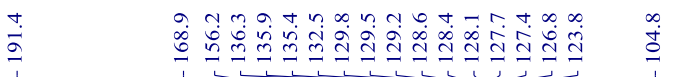

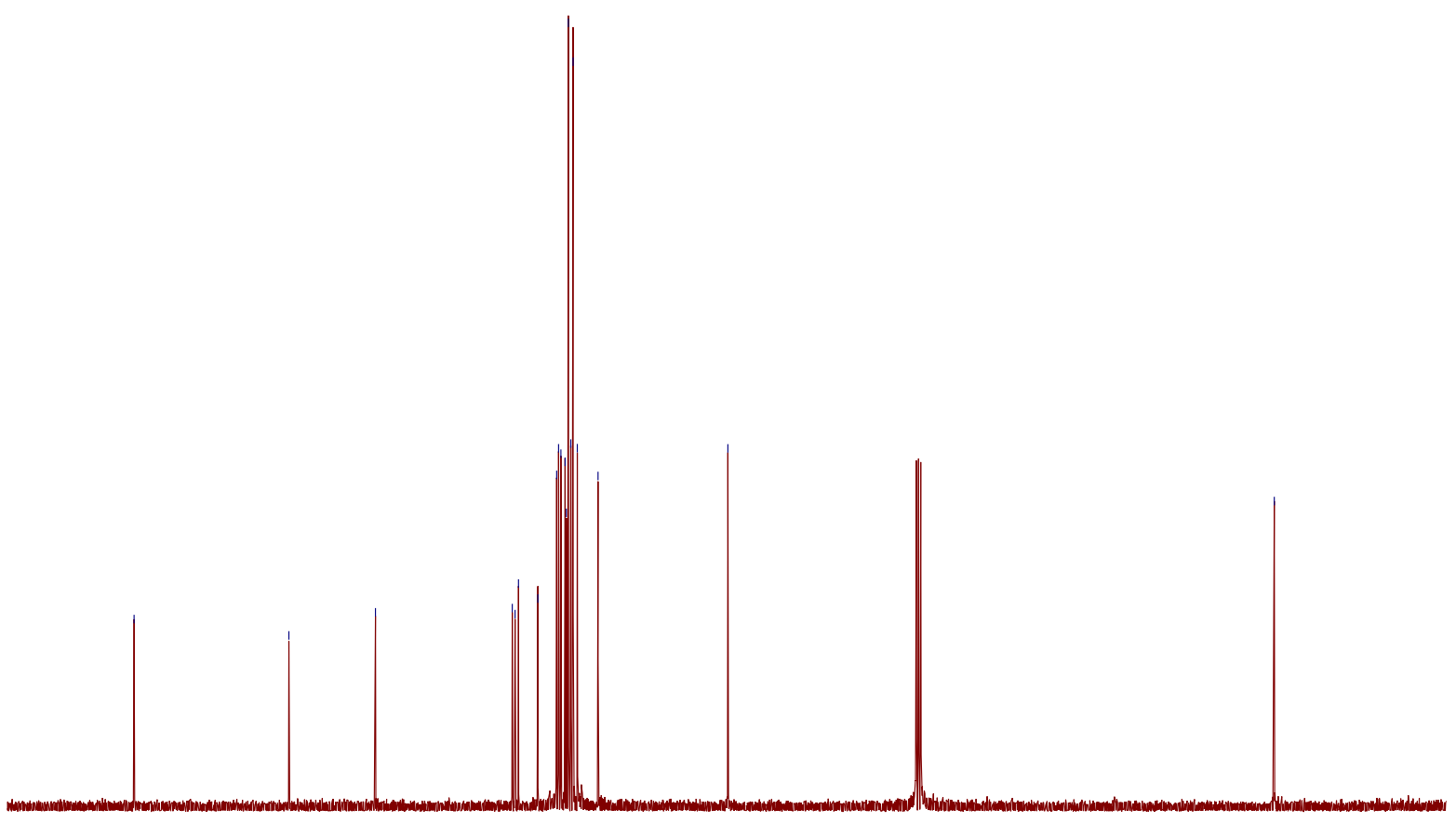

$\begin{array}{rlllllllll}200 & 190 & 180 & 170 & 160 & 150 & 140 & 130 & 120 & 110 \begin{array}{r}100 \\ \text { f1 (ppm) }\end{array}\end{array}$

${ }^{1} \mathrm{H}$ and ${ }^{13} \mathrm{C}$ NMR spectra for product $3 \mathrm{~s}\left(\mathrm{CDCl}_{3}\right)$ 

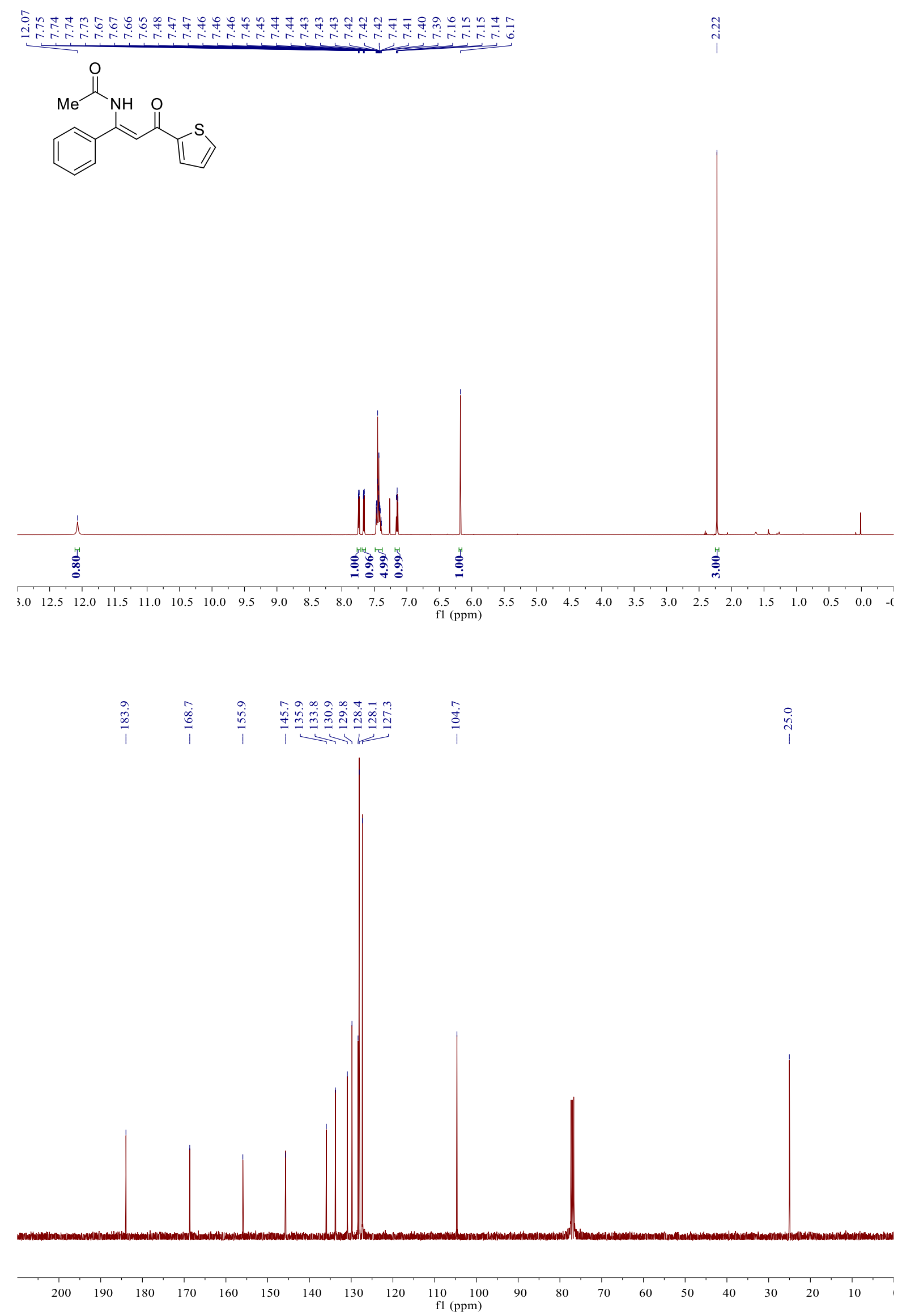

${ }^{1} \mathrm{H}$ and ${ }^{13} \mathrm{C}$ NMR spectra for product $3 \mathrm{t}\left(\mathrm{CDCl}_{3}\right)$ 


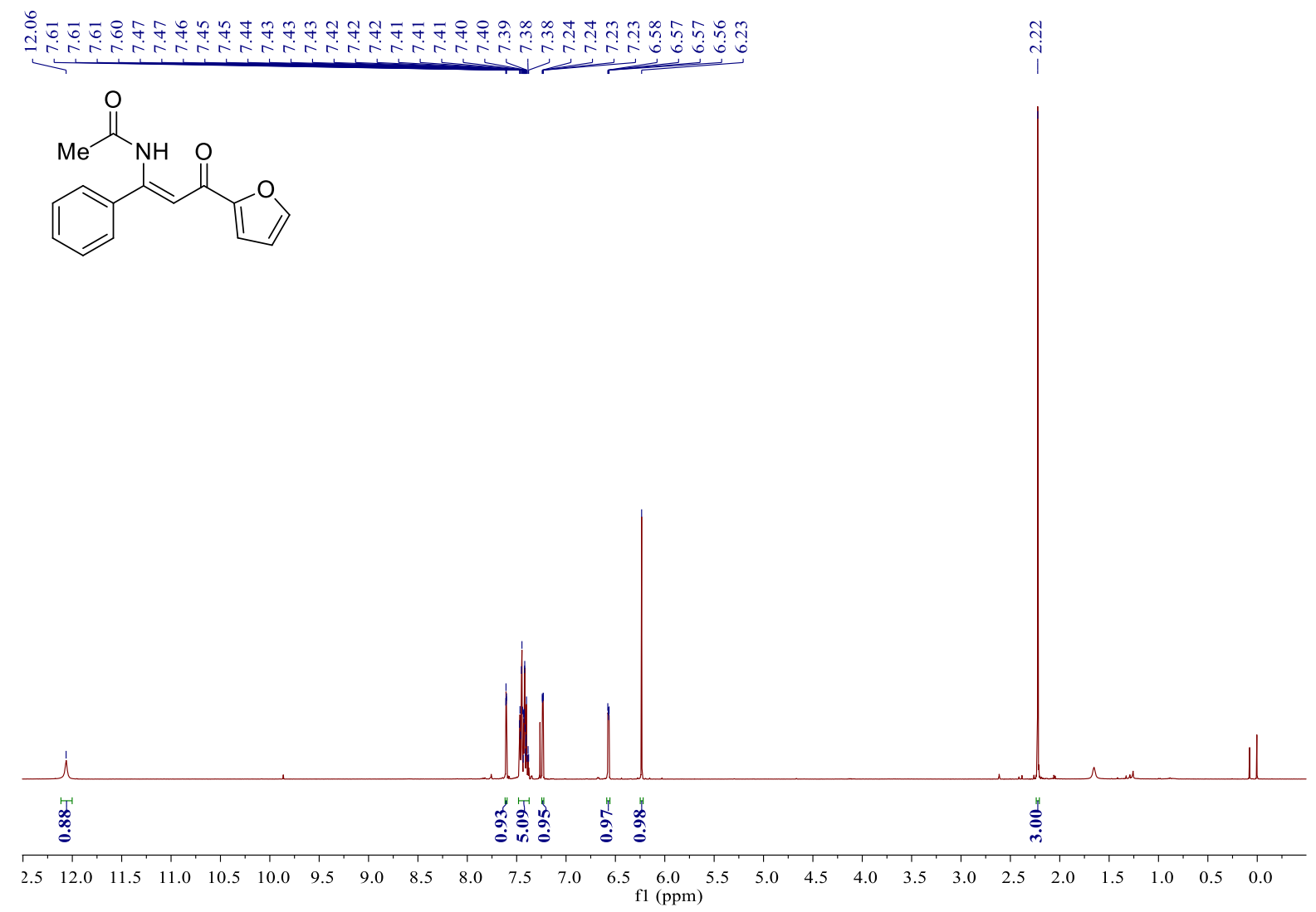

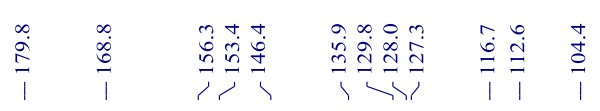

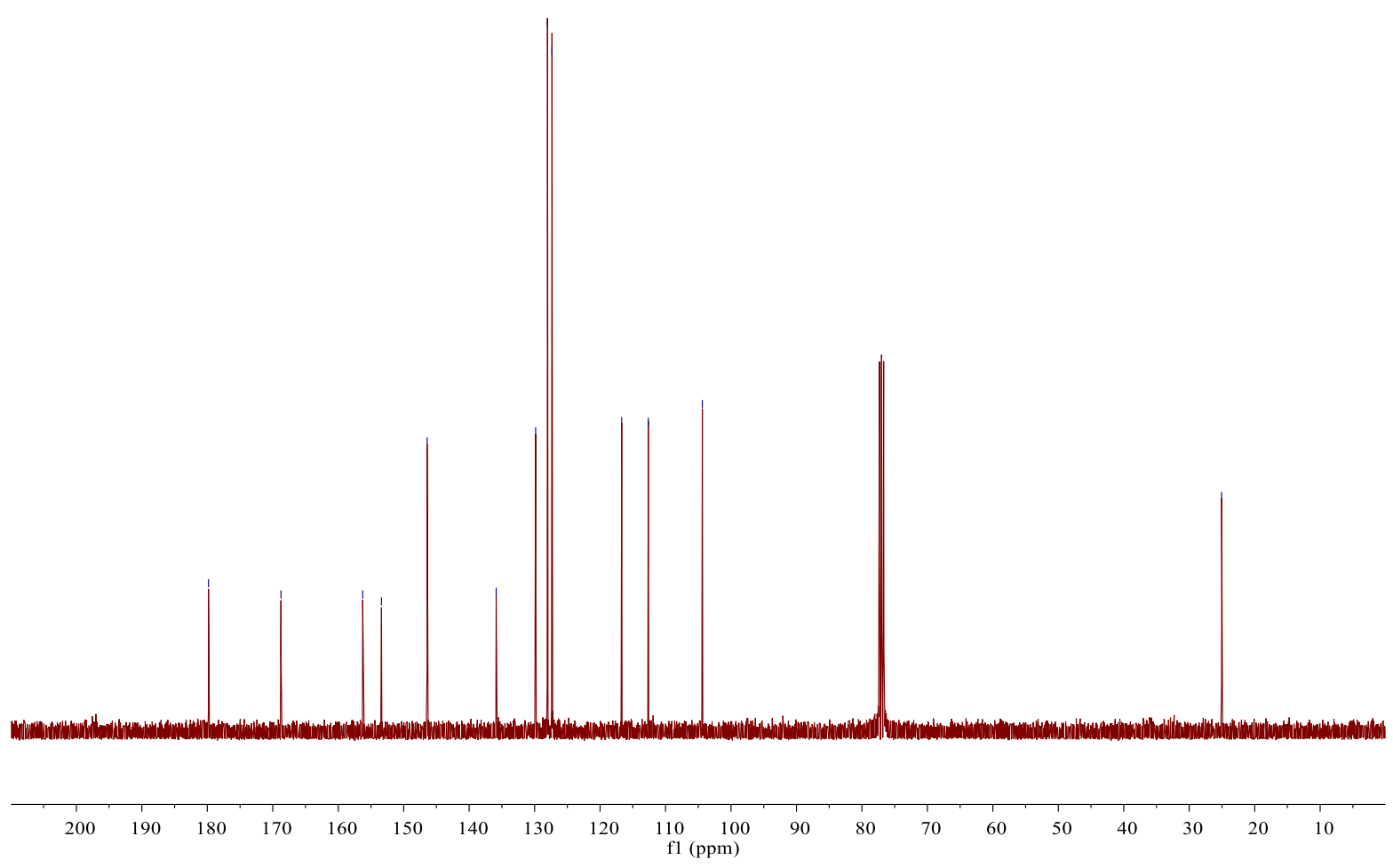

${ }^{1} \mathrm{H}$ and ${ }^{13} \mathrm{C}$ NMR spectra for product $3 \mathrm{u}\left(\mathrm{CDCl}_{3}\right)$ 

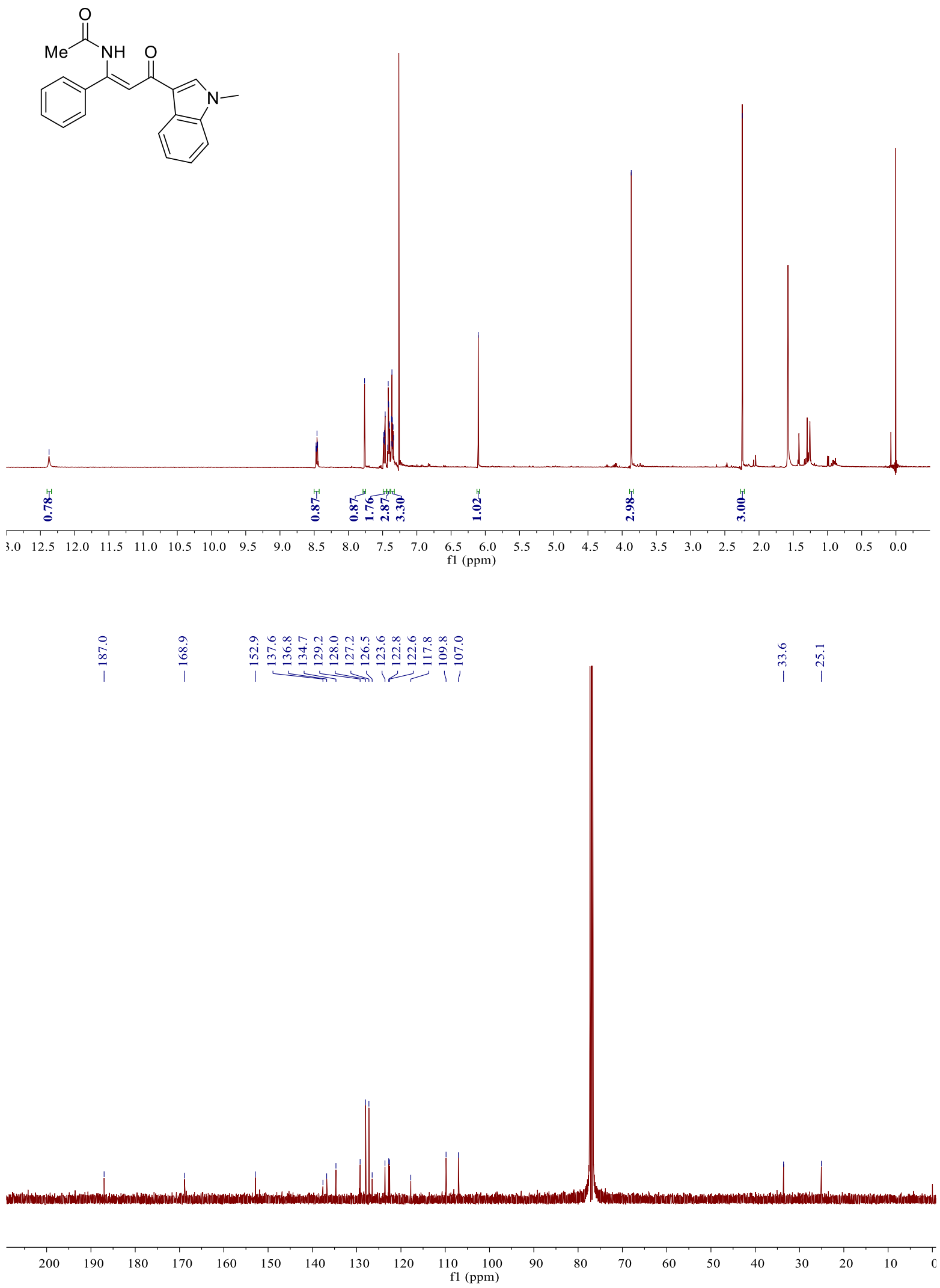

${ }^{1} \mathrm{H}$ and ${ }^{13} \mathrm{C}$ NMR spectra for product $3 \mathrm{v}\left(\mathrm{CDCl}_{3}\right)$ 

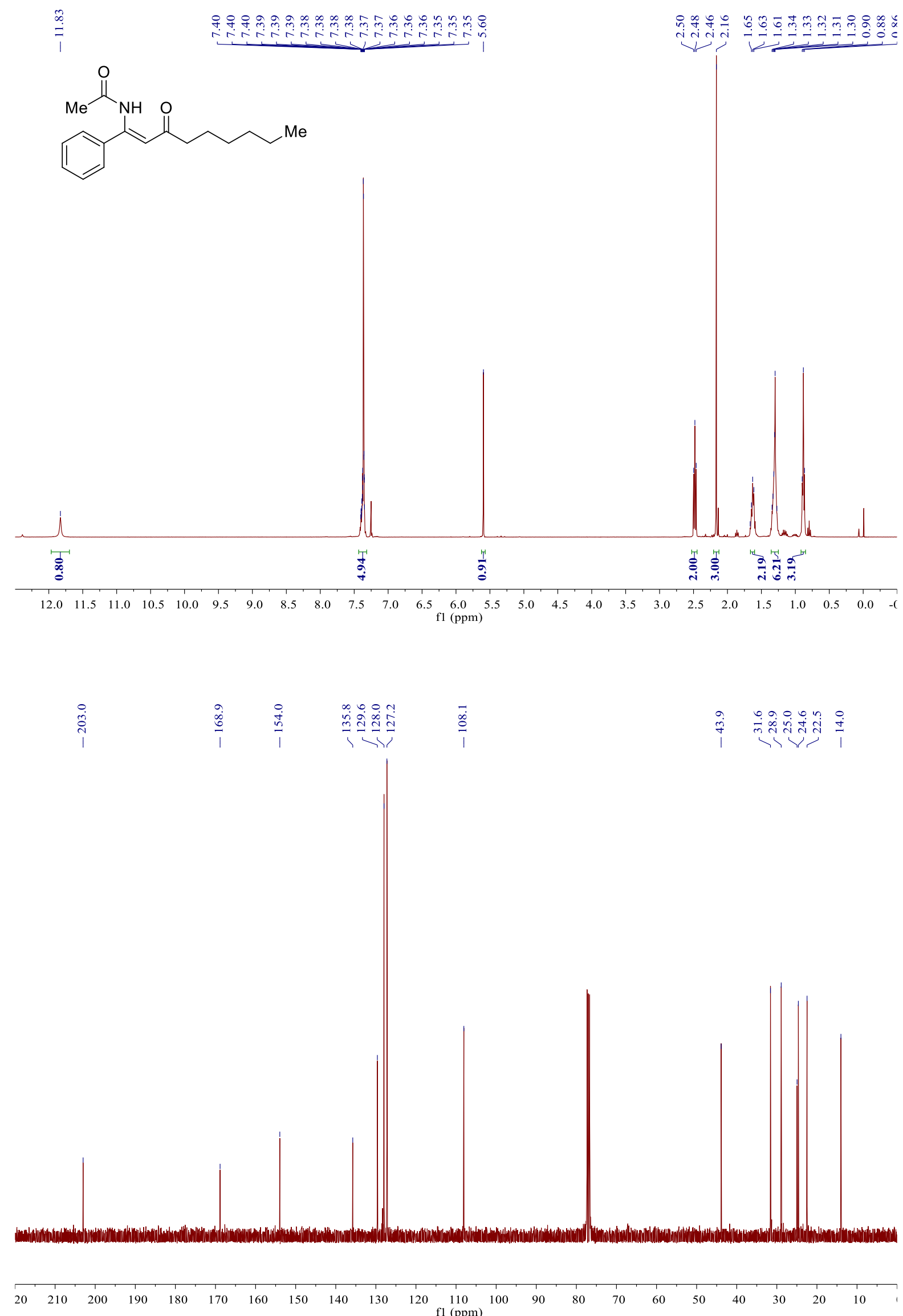

${ }^{1} \mathrm{H}$ and ${ }^{13} \mathrm{C}$ NMR spectra for product $3 \mathrm{w}\left(\mathrm{CDCl}_{3}\right)$ 


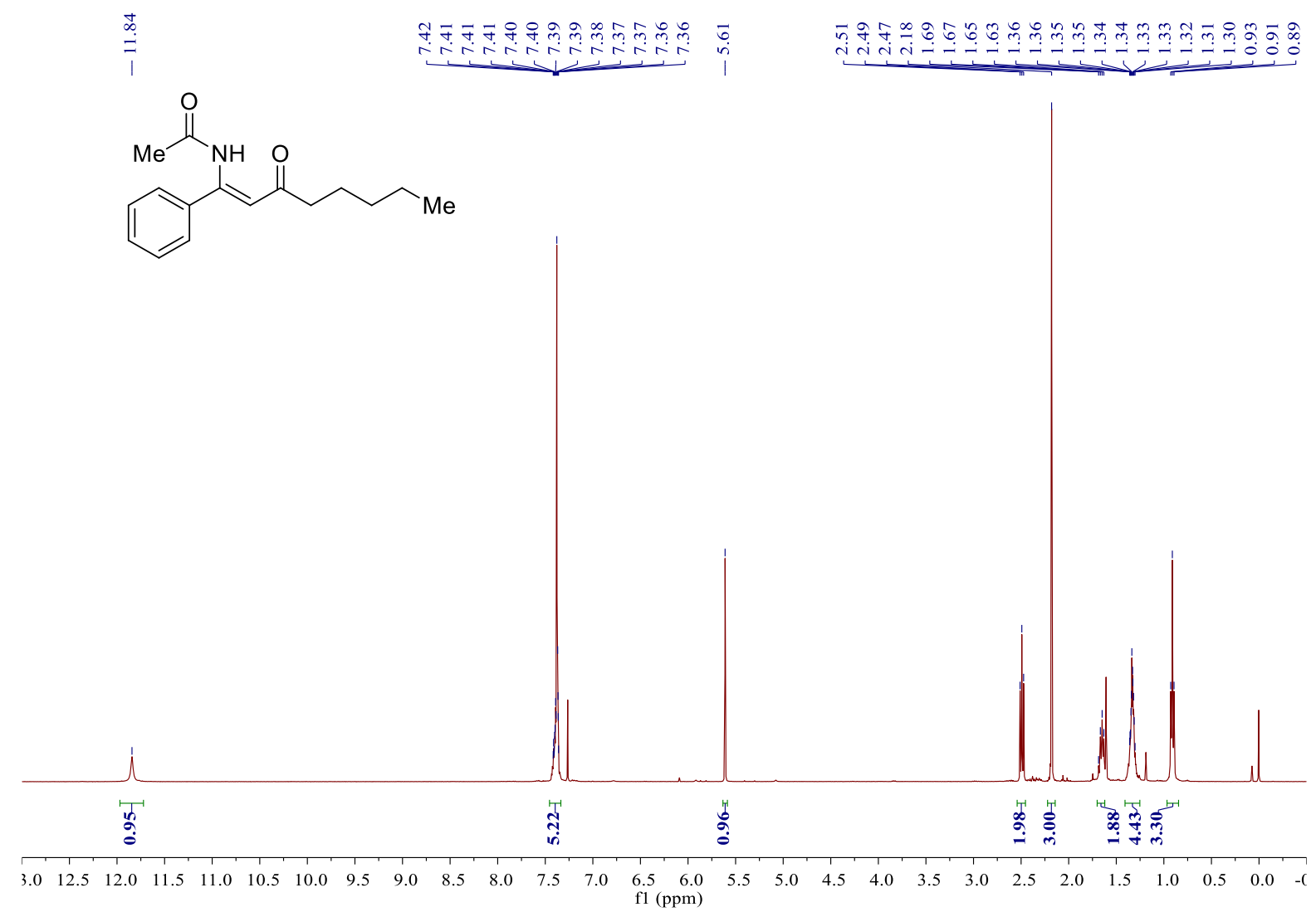

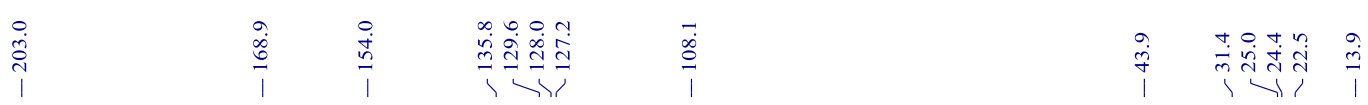

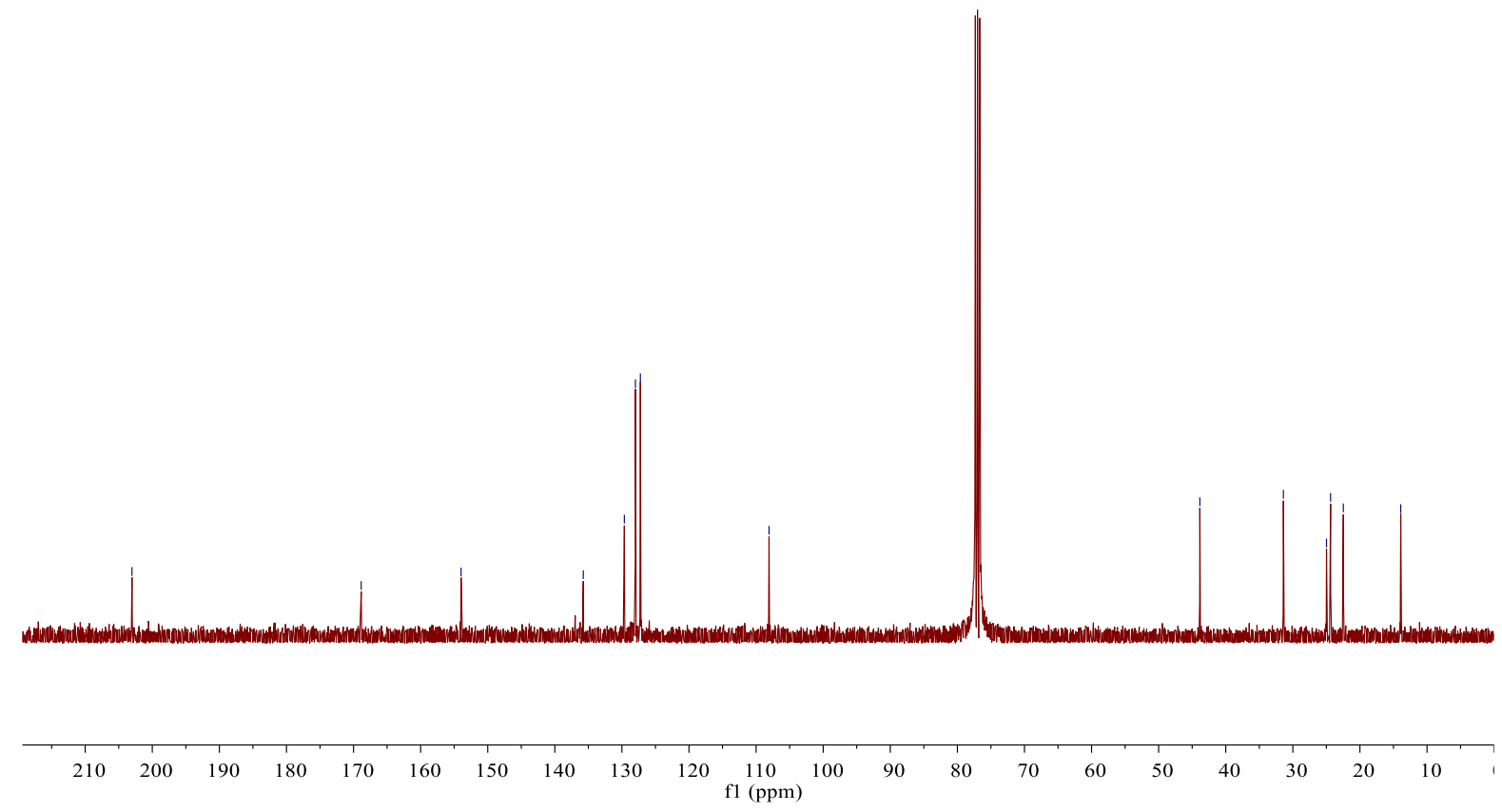

${ }^{1} \mathrm{H}$ and ${ }^{13} \mathrm{C}$ NMR spectra for product $3 \times\left(\mathrm{CDCl}_{3}\right)$ 
$\stackrel{\vec{\infty}}{\vec{\perp}}$

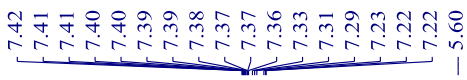

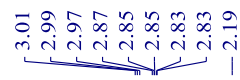
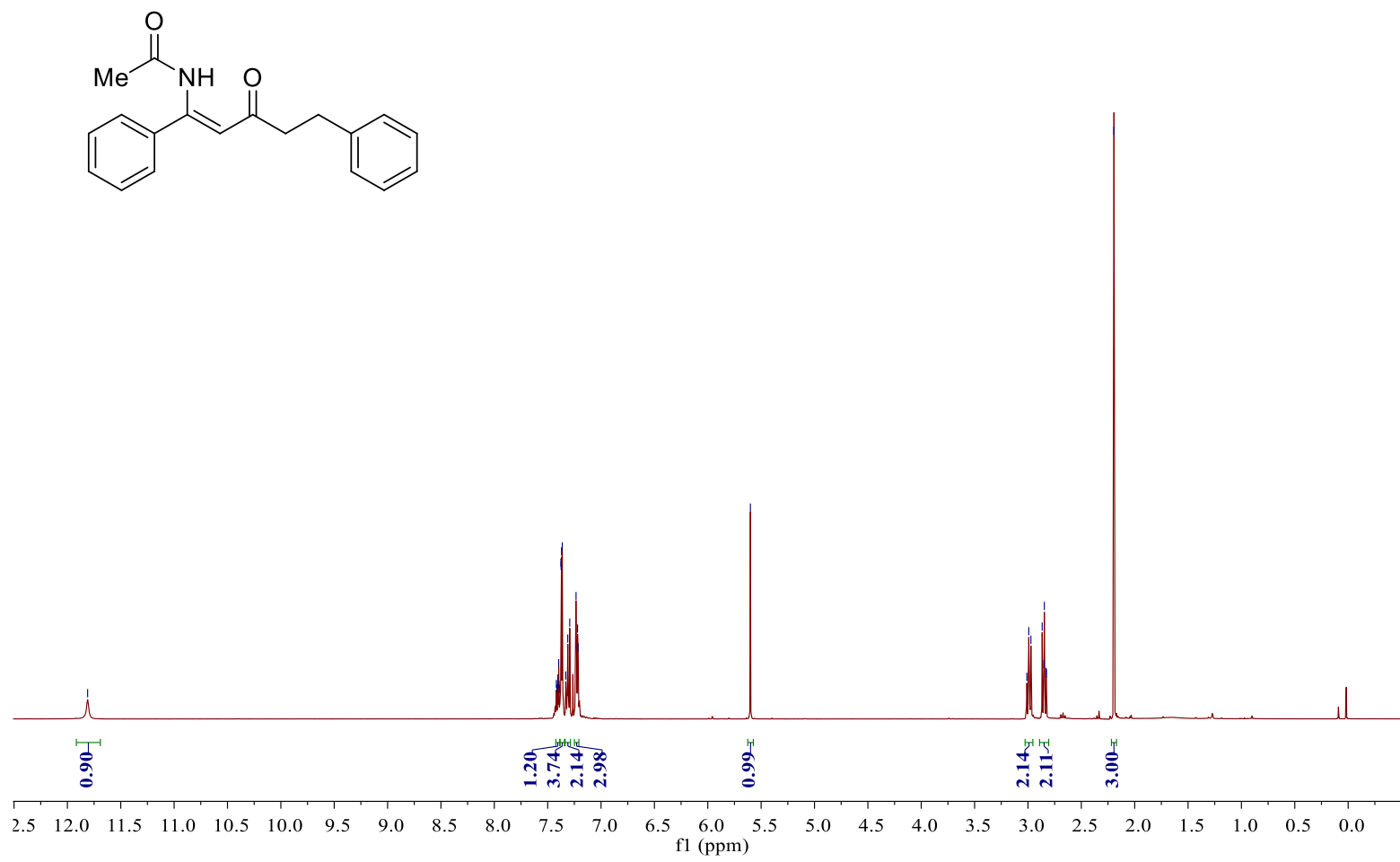

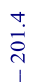

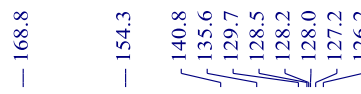

$\stackrel{\stackrel{2}{\circ}}{1}$

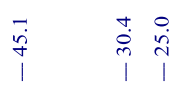
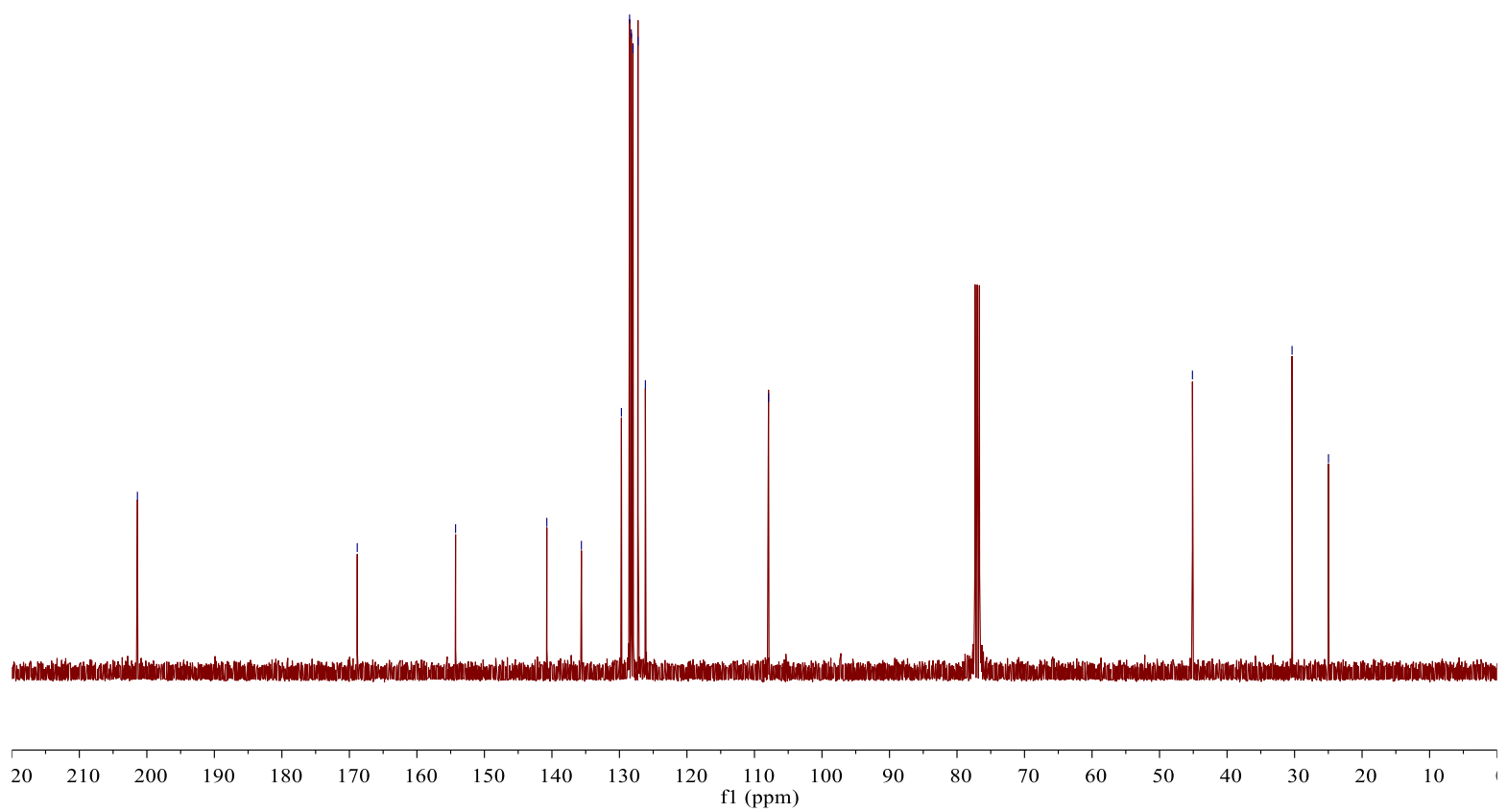

${ }^{1} \mathrm{H}$ and ${ }^{13} \mathrm{C}$ NMR spectra for product $3 \mathrm{y}\left(\mathrm{CDCl}_{3}\right)$

S75 

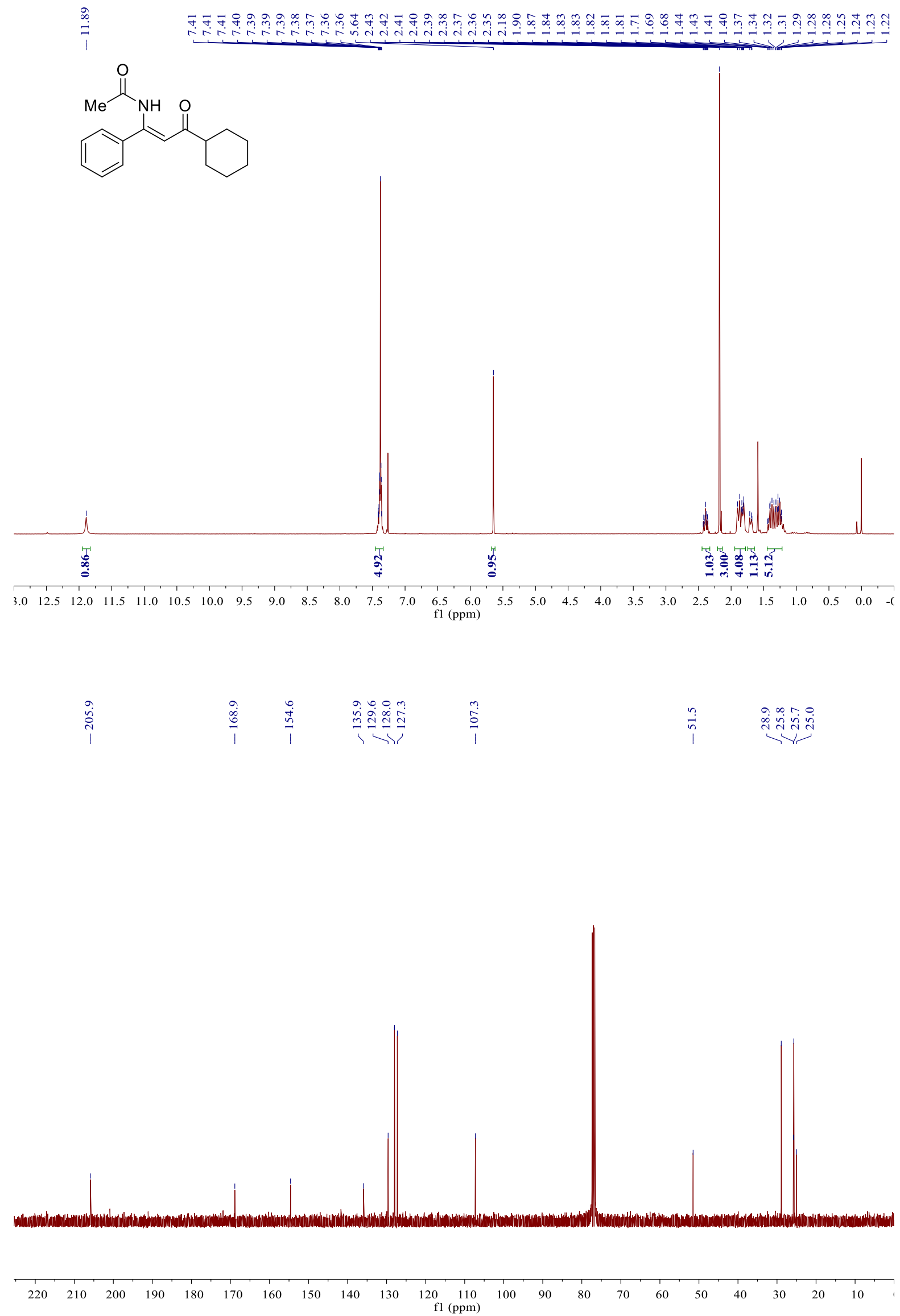

${ }^{1} \mathrm{H}$ and ${ }^{13} \mathrm{C}$ NMR spectra for product $3 \mathrm{z}\left(\mathrm{CDCl}_{3}\right)$ 


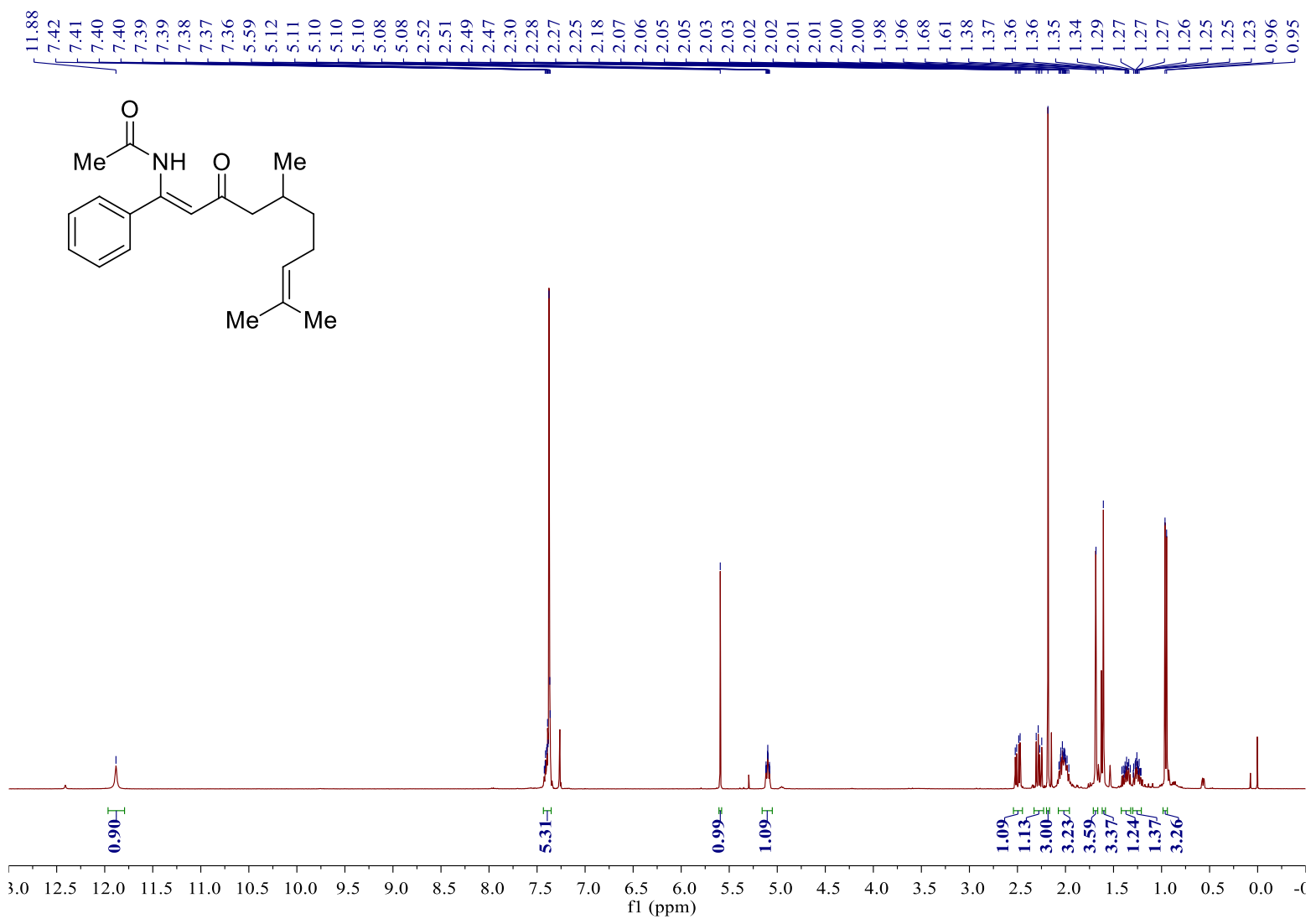

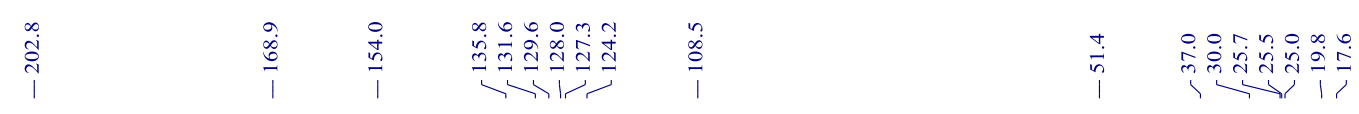

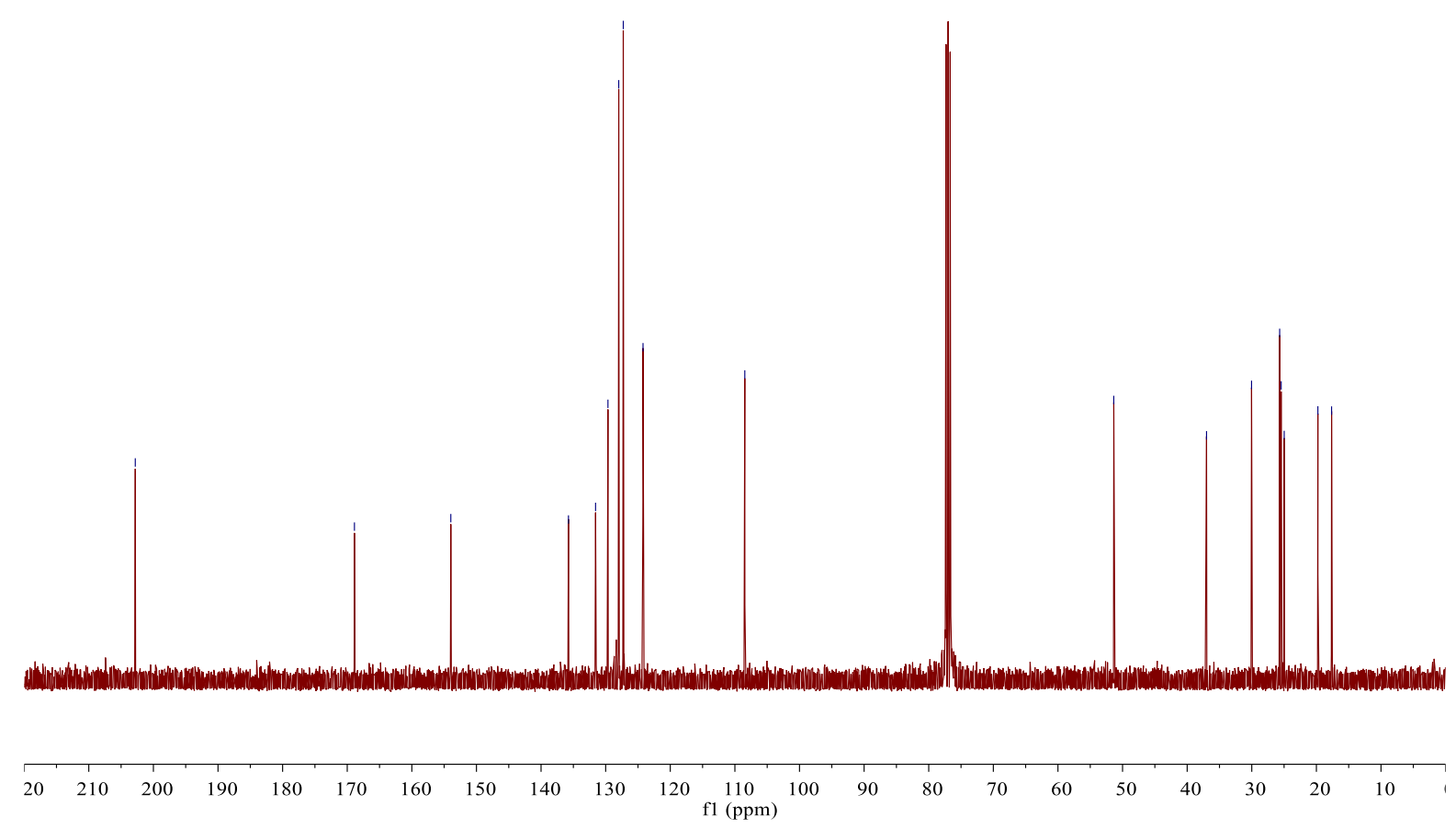

${ }^{1} \mathrm{H}$ and ${ }^{13} \mathrm{C}$ NMR spectra for product 3aa $\left(\mathrm{CDCl}_{3}\right)$ 
<smiles>CC(=O)N/C(=C\C(=O)c1ccc(C(=O)OC2CC([N+](=O)[O-])CCC2C(C)C)cc1)c1ccccc1</smiles>
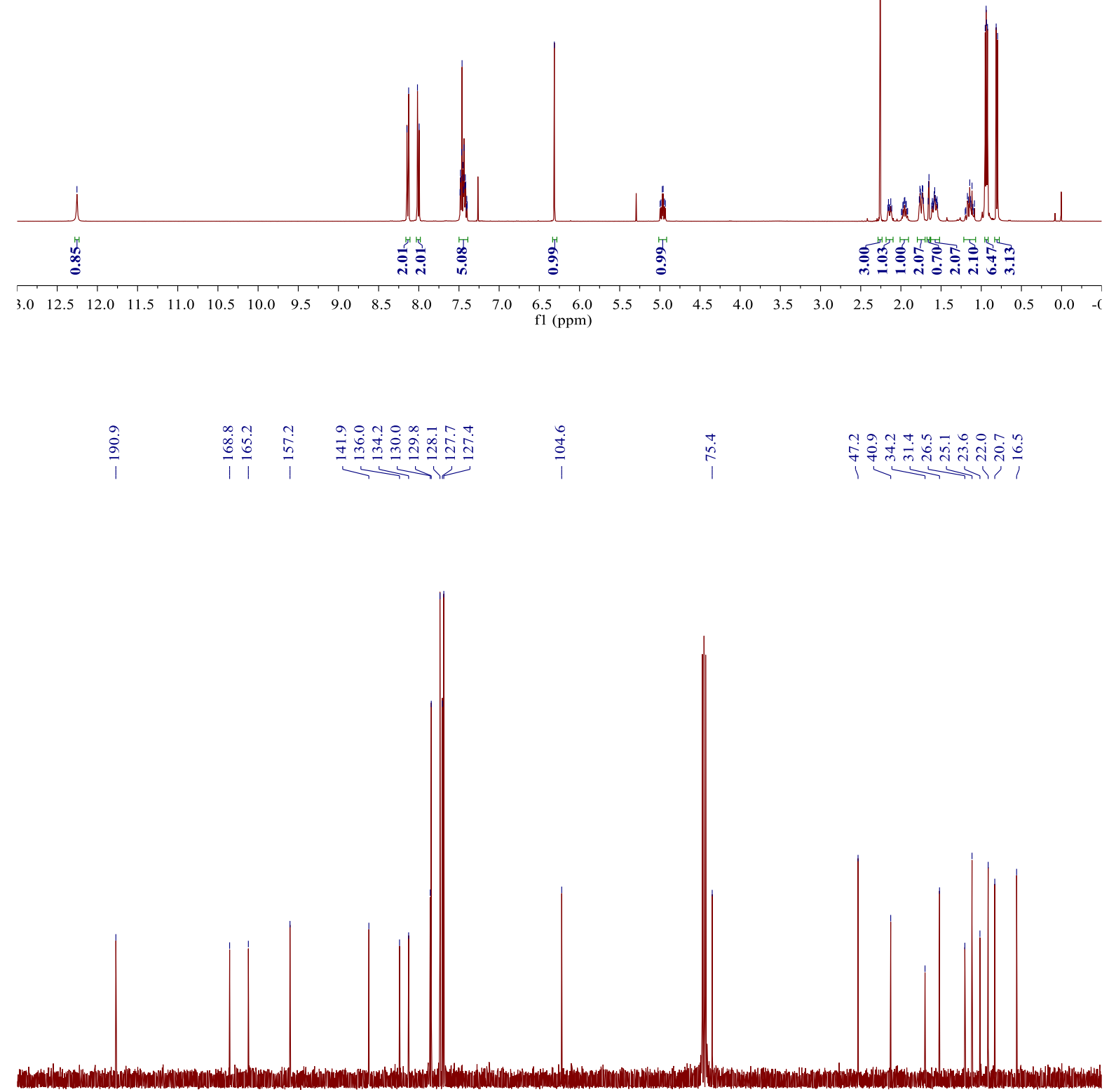

$\begin{array}{rllllllllll}10 & 200 & 190 & 180 & 170 & 160 & 150 & 140 & 130 & 120 & \begin{array}{c}110 \\ \text { f1 (ppm) }\end{array}\end{array}$

${ }^{1} \mathrm{H}$ and ${ }^{13} \mathrm{C}$ NMR spectra for product $3 \mathrm{ab}\left(\mathrm{CDCl}_{3}\right)$ 

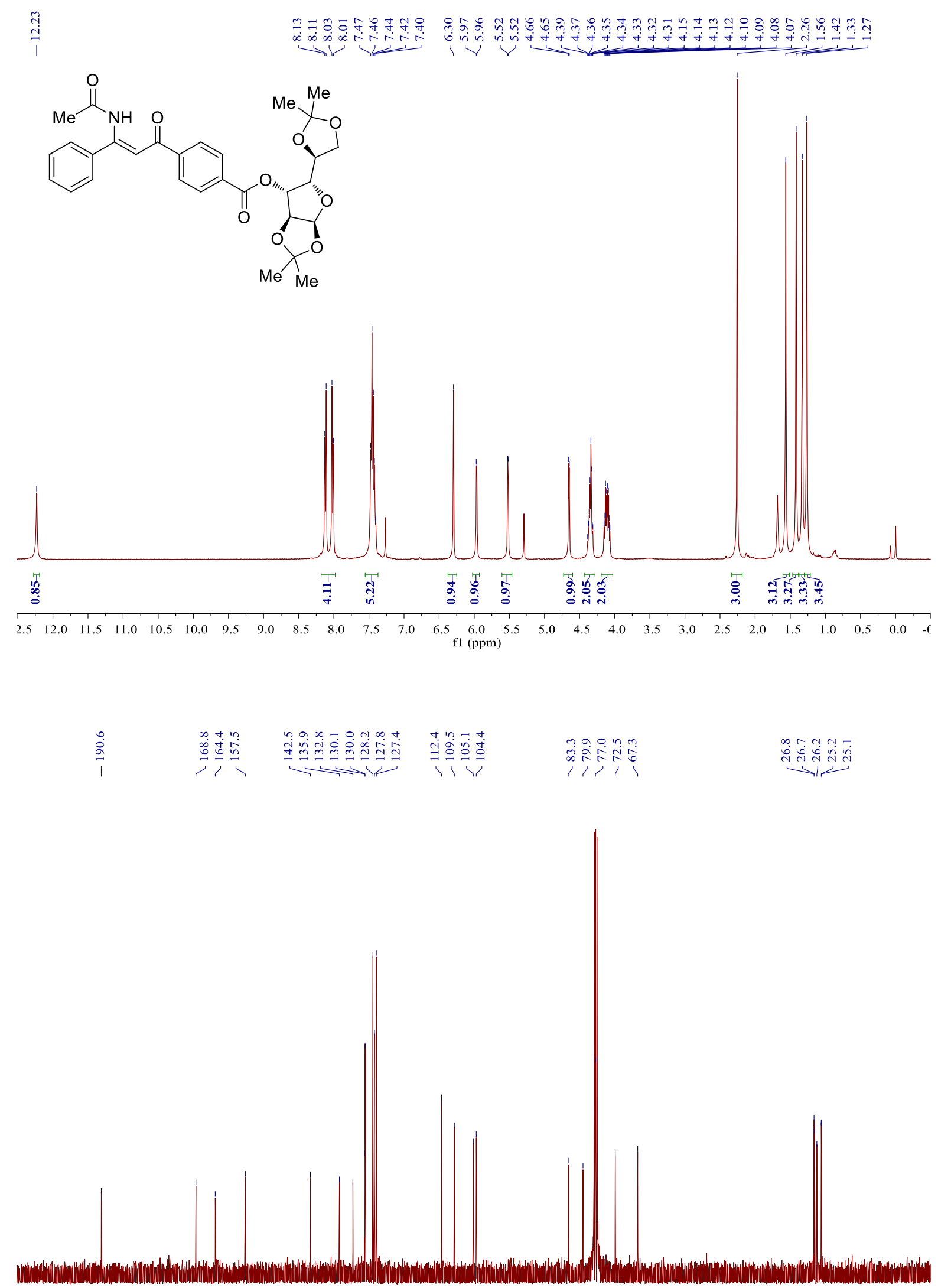

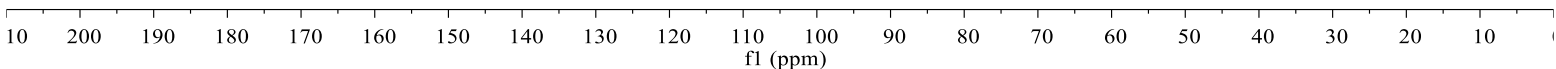

${ }^{1} \mathrm{H}$ and ${ }^{13} \mathrm{C}$ NMR spectra for product $3 \mathrm{ac}\left(\mathrm{CDCl}_{3}\right)$ 


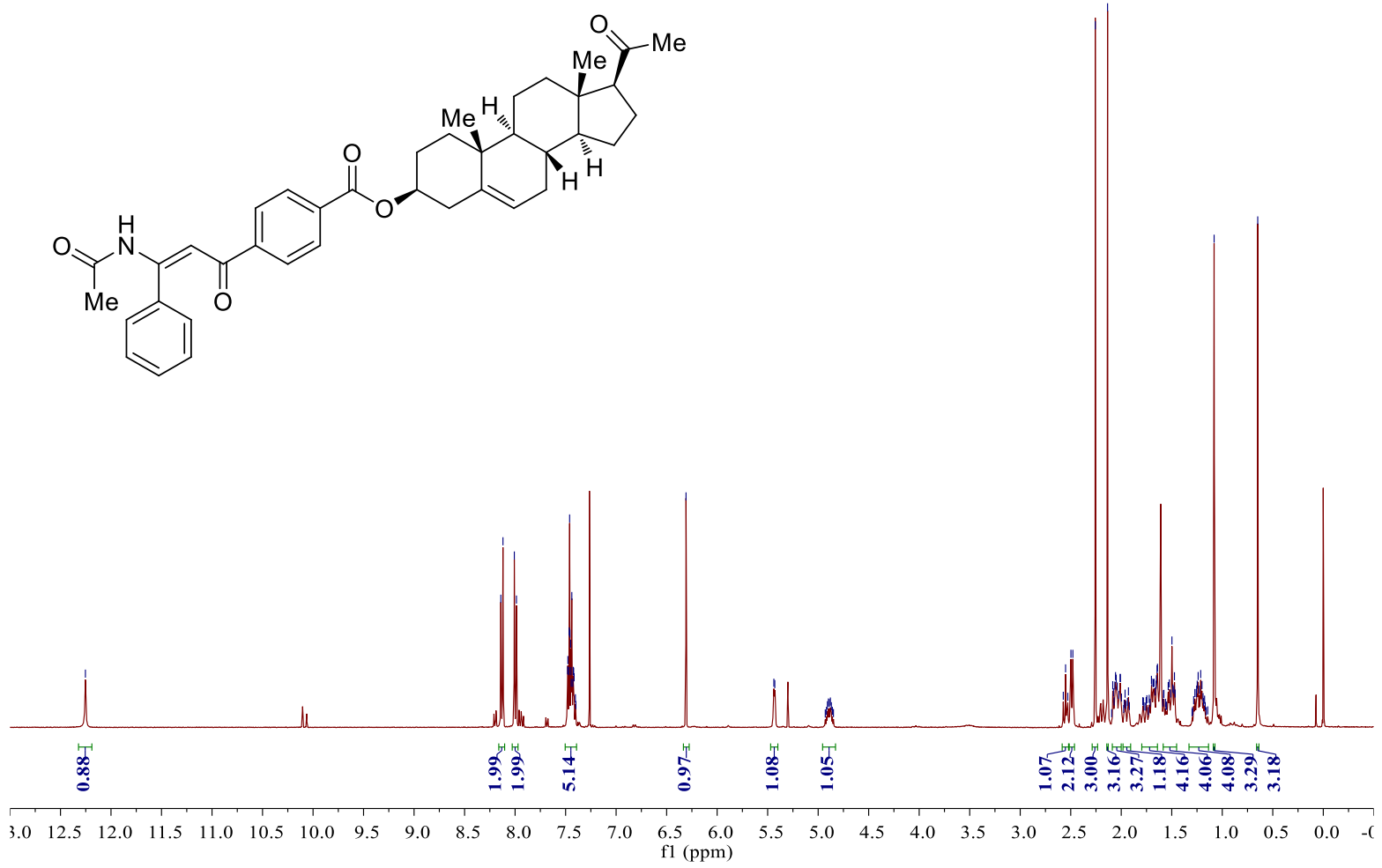

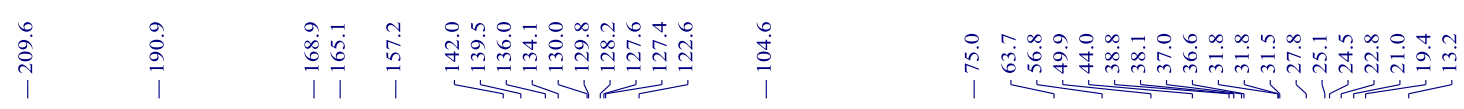

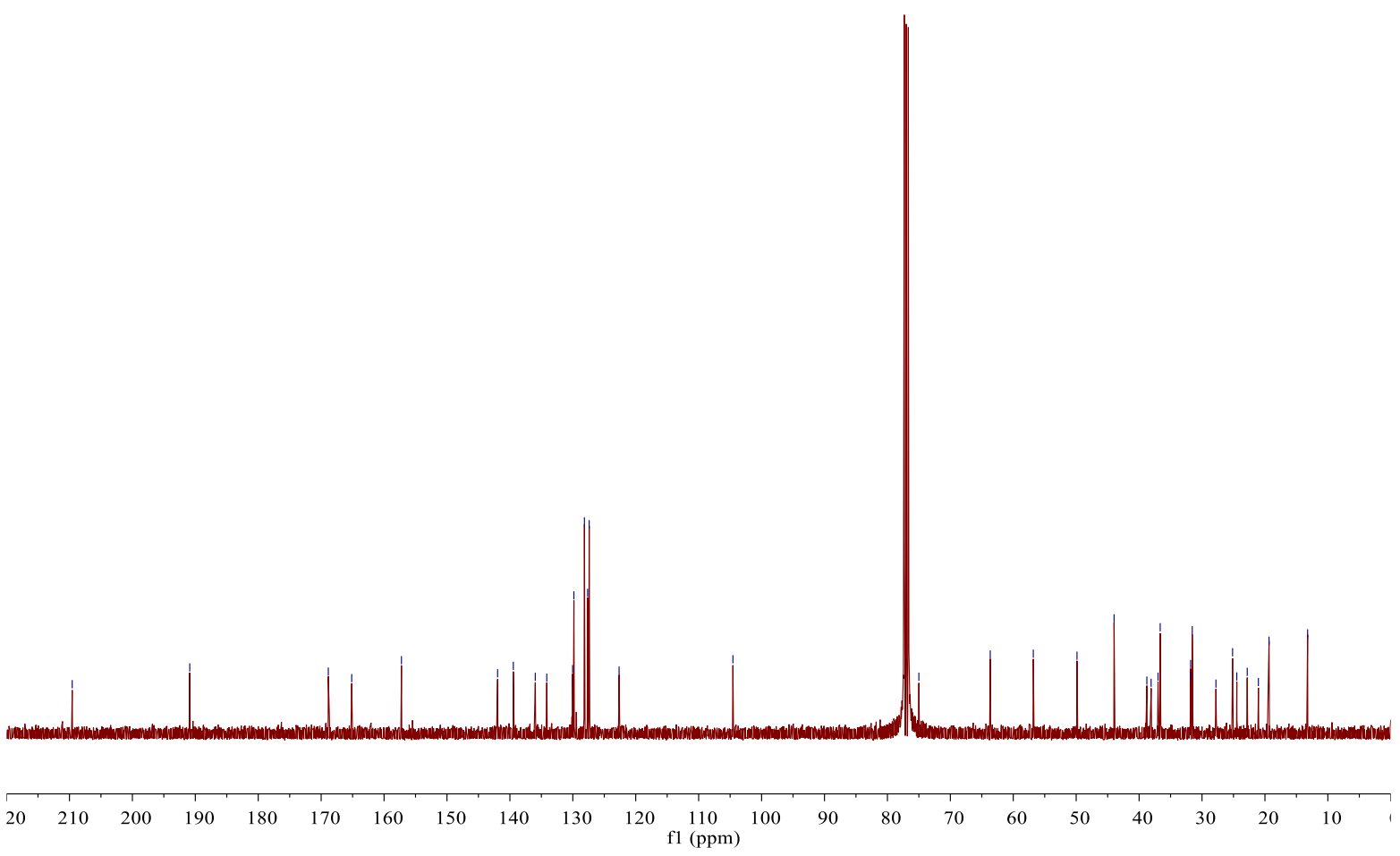

${ }^{1} \mathrm{H}$ and ${ }^{13} \mathrm{C}$ NMR spectra for product $3 \mathrm{ad}\left(\mathrm{CDCl}_{3}\right)$ 


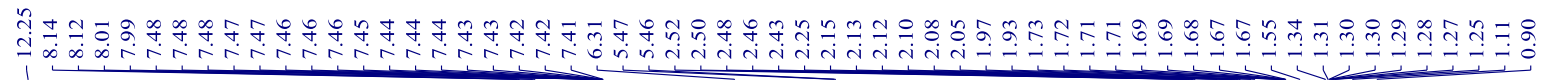<smiles>CC(=O)N/C(=C/C(=O)c1ccc(C(=O)O[C@H]2CC[C@@]3(C)C(=CC[C@@H]4[C@@H]5CCC(=O)[C@@]5(C)CC[C@H]43)C2)cc1)c1ccccc1</smiles>

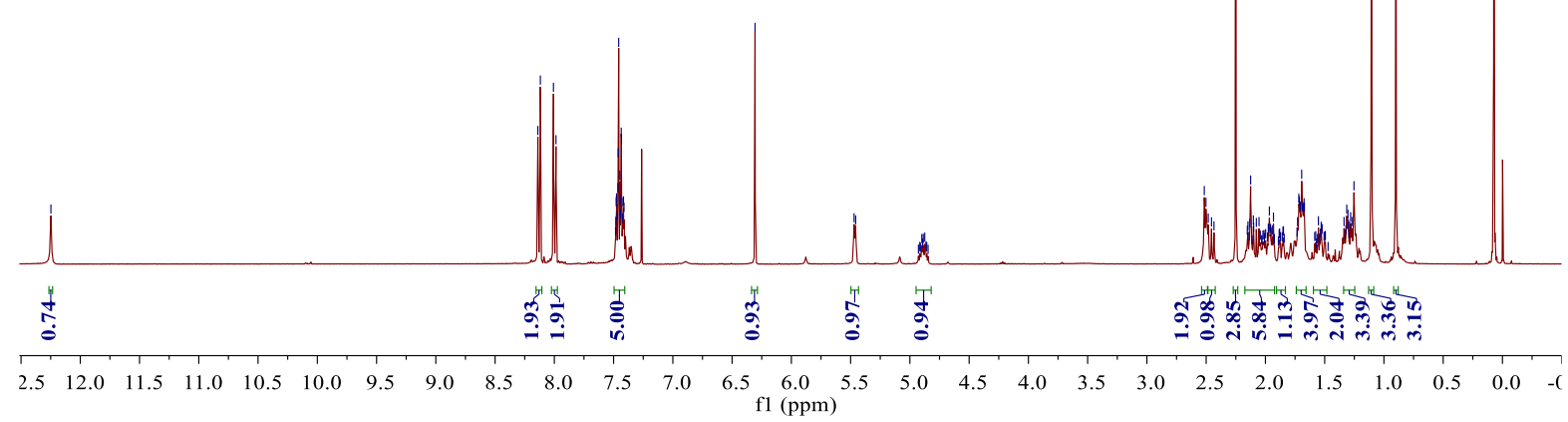

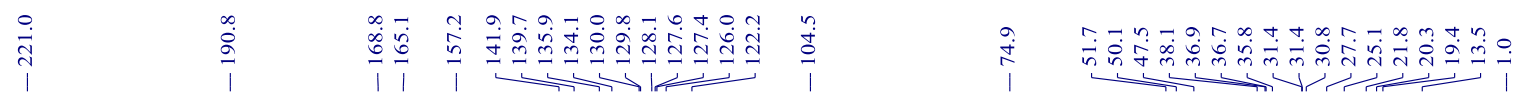

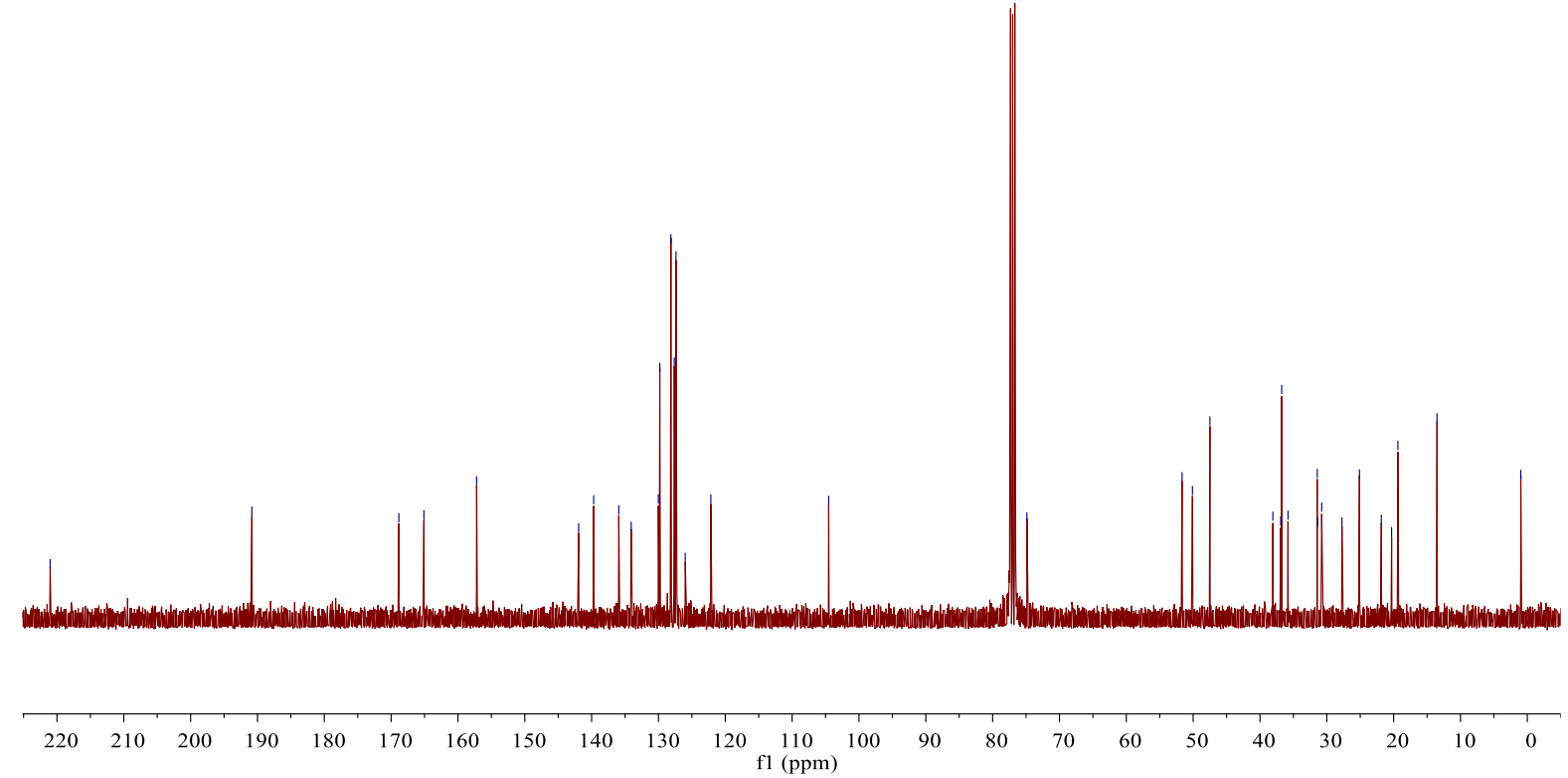

${ }^{1} \mathrm{H}$ and ${ }^{13} \mathrm{C}$ NMR spectra for product $3 \mathrm{ae}\left(\mathrm{CDCl}_{3}\right)$ 


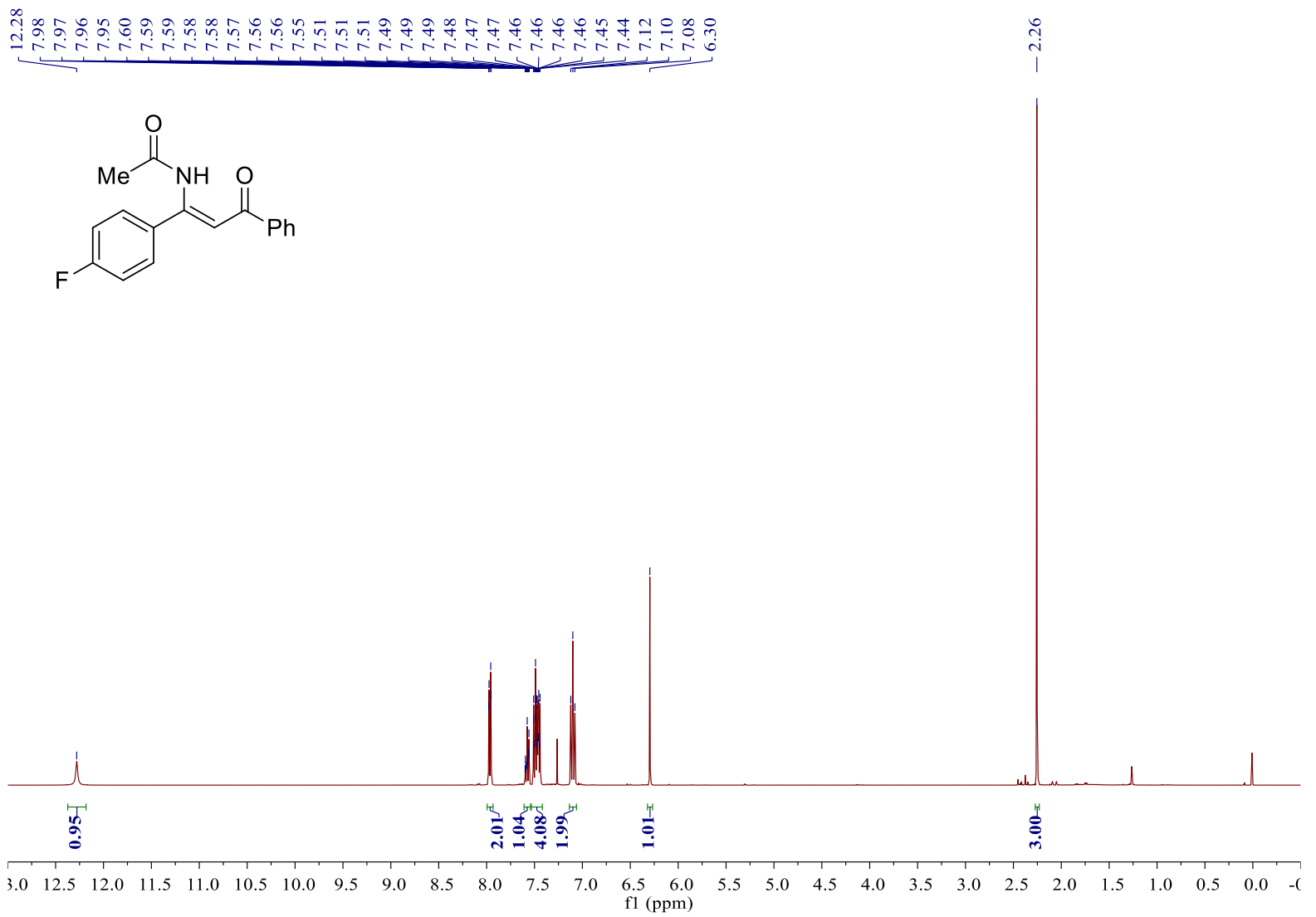

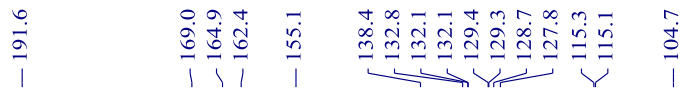

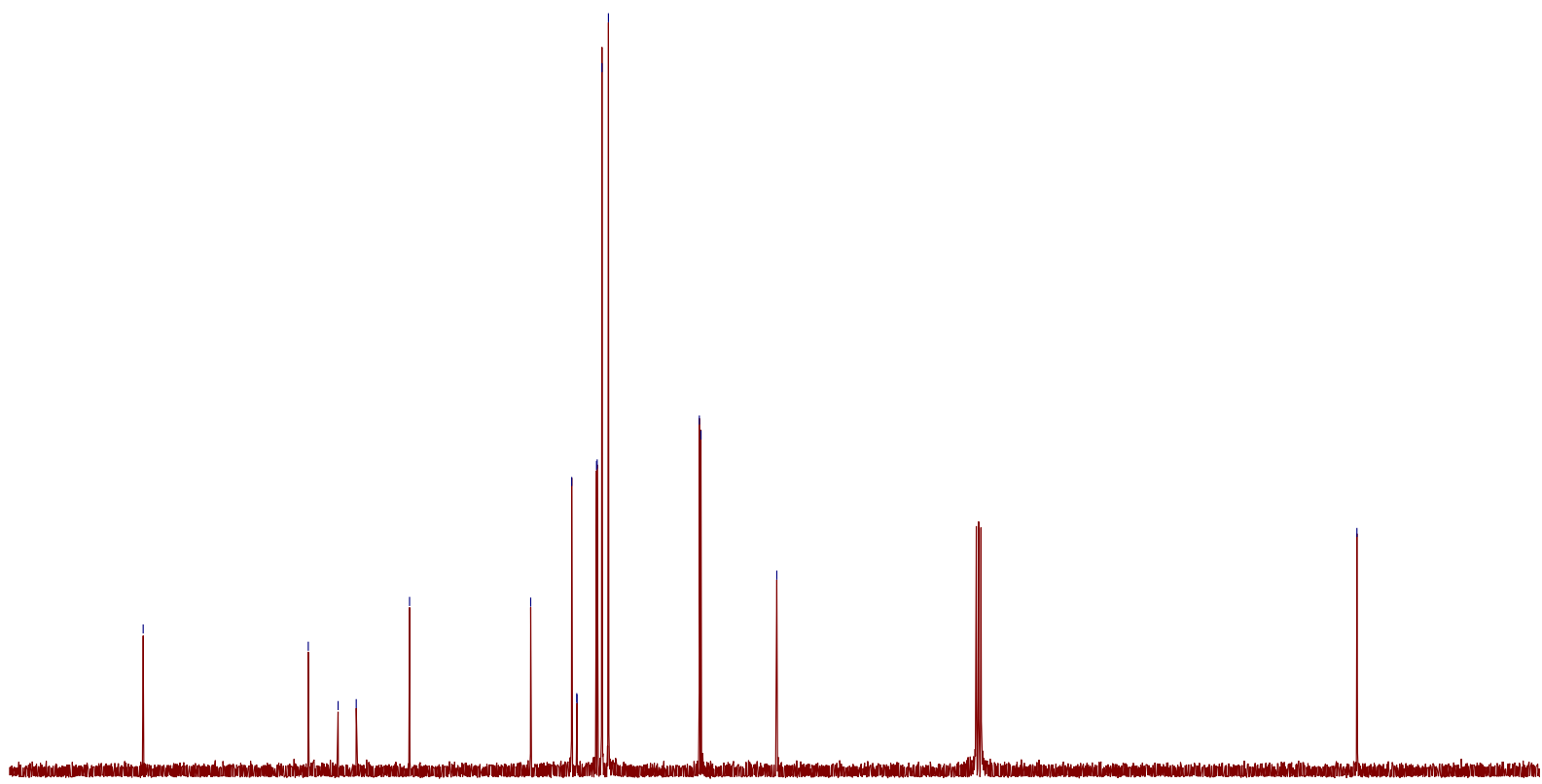

$\begin{array}{llllllllllllllllllll}200 & 190 & 180 & 170 & 160 & 150 & 140 & 130 & 120 & \begin{array}{c}110 \\ \mathrm{fl}(\mathrm{ppm})\end{array} & 90 & 80 & 70 & 60 & 50 & 40 & 30 & 20 & 10\end{array}$ 


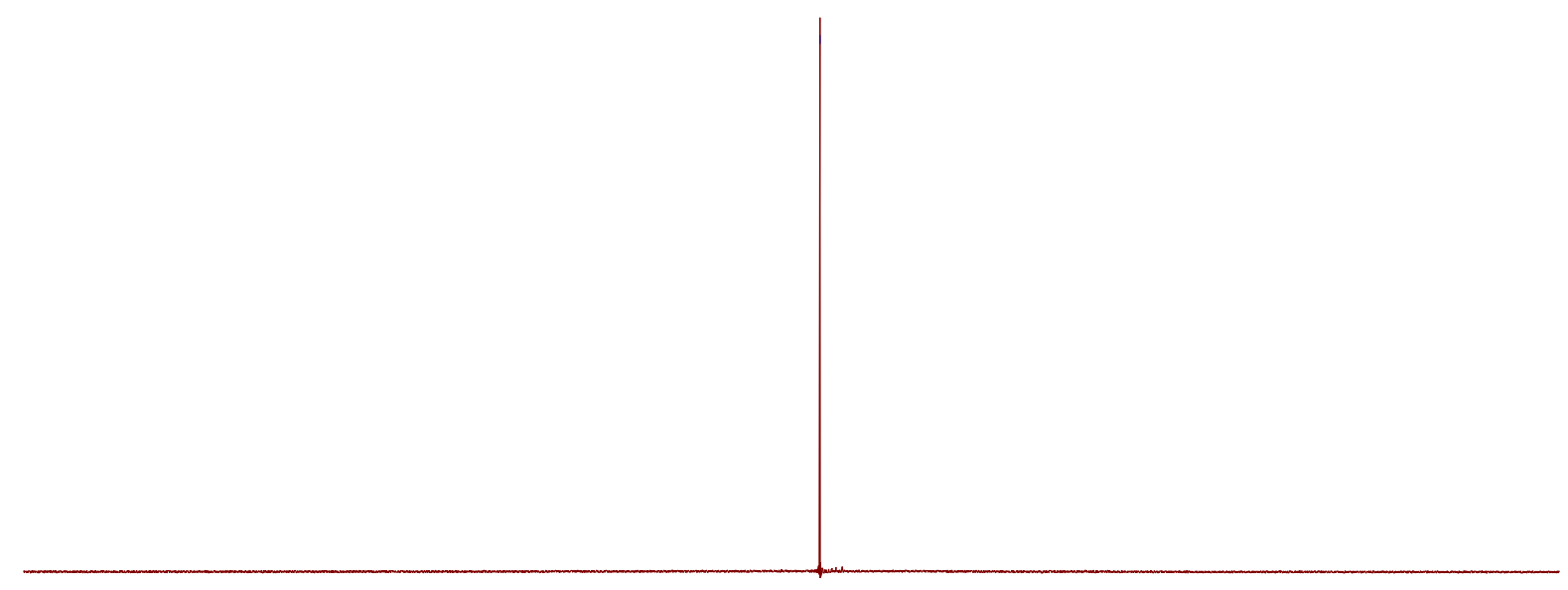

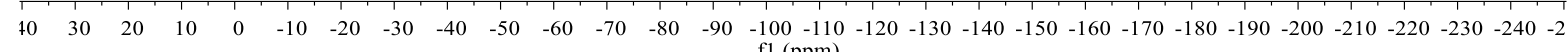
fl (ppm)

${ }^{1} \mathrm{H},{ }^{19} \mathrm{~F}$, and ${ }^{13} \mathrm{C}$ NMR spectra for product $4 \mathrm{a}\left(\mathrm{CDCl}_{3}\right)$ 


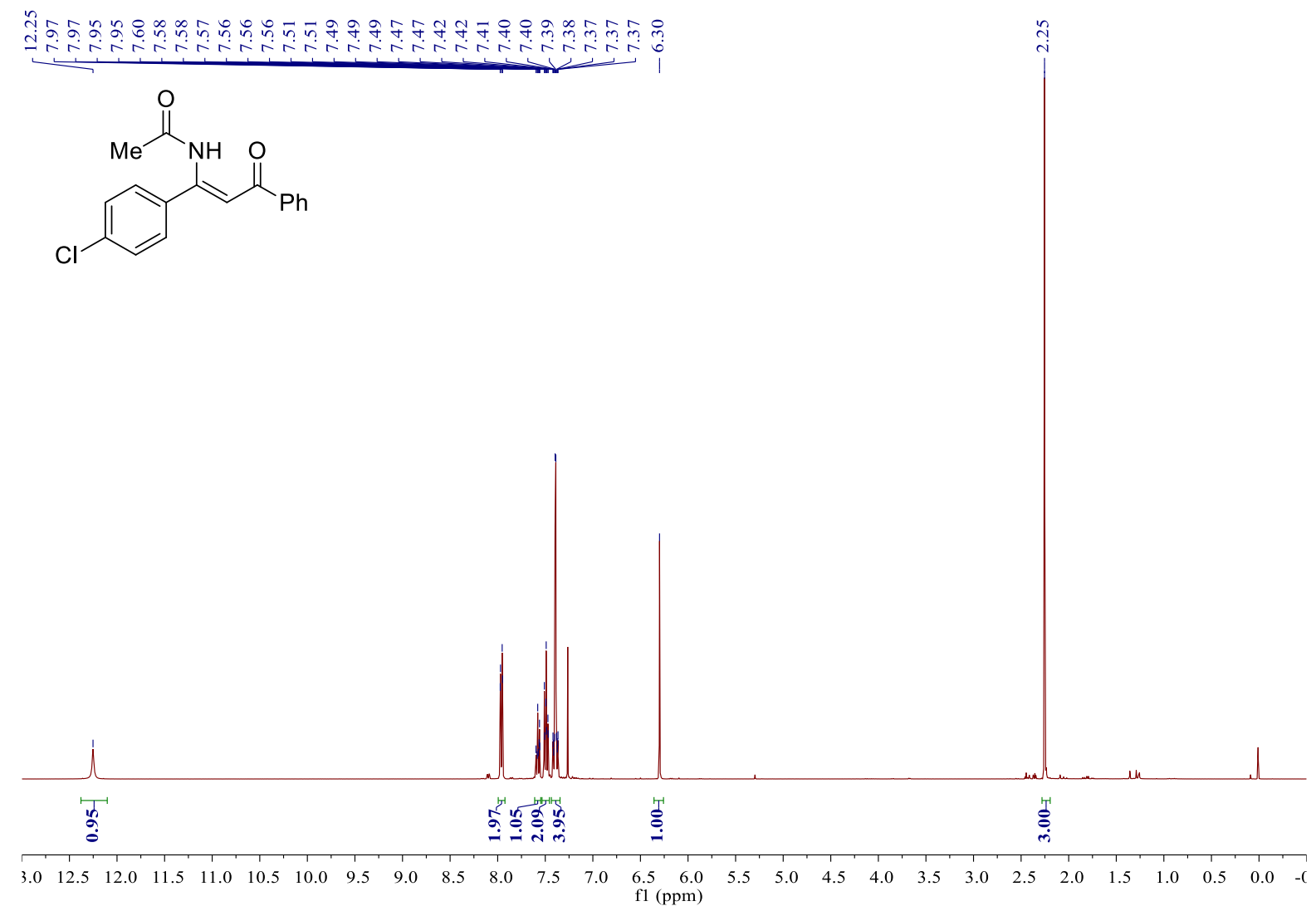

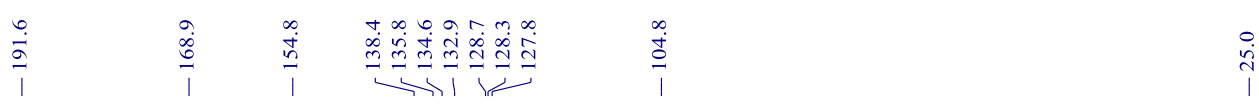

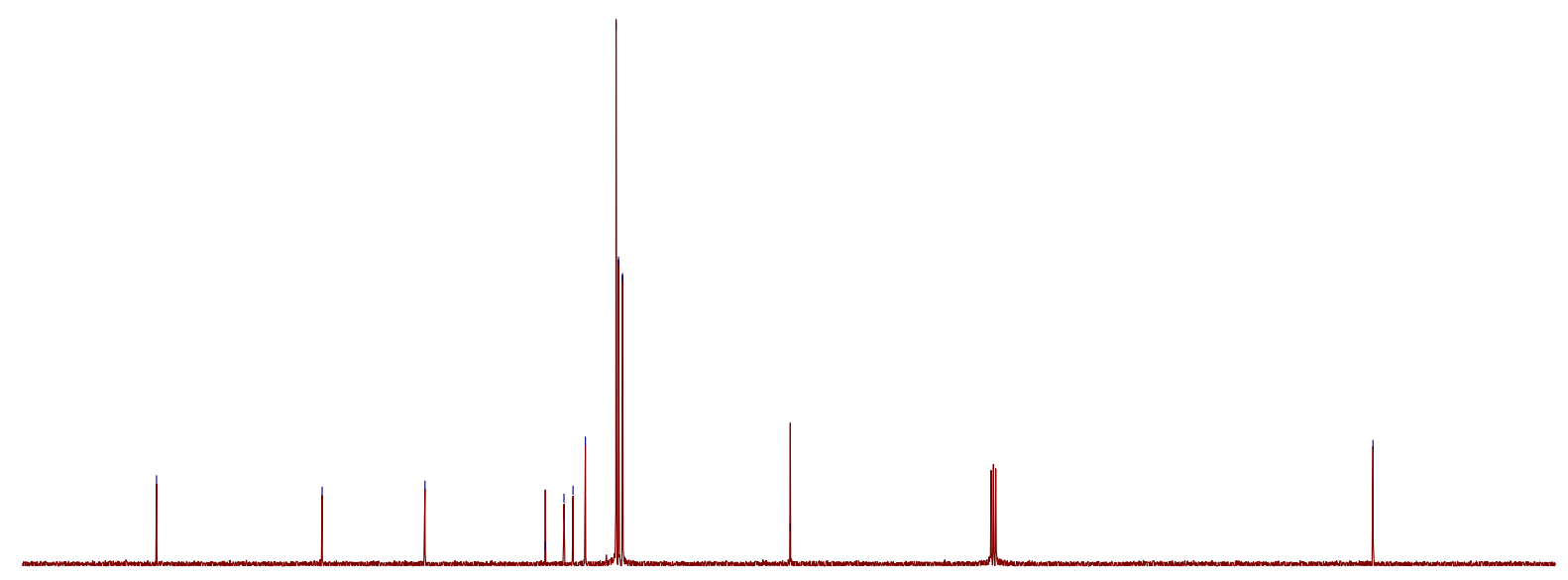

$\begin{array}{lllllllllllllllllllllll}10 & 200 & 190 & 180 & 170 & 160 & 150 & 140 & 130 & 120 & \begin{array}{c}110 \\ \mathrm{fl}(\mathrm{ppm})\end{array} & 90 & 80 & 70 & 60 & 50 & 40 & 30 & 20 & 10 & 10\end{array}$

${ }^{1} \mathrm{H}$ and ${ }^{13} \mathrm{C}$ NMR spectra for product $4 \mathrm{~b}\left(\mathrm{CDCl}_{3}\right)$ 


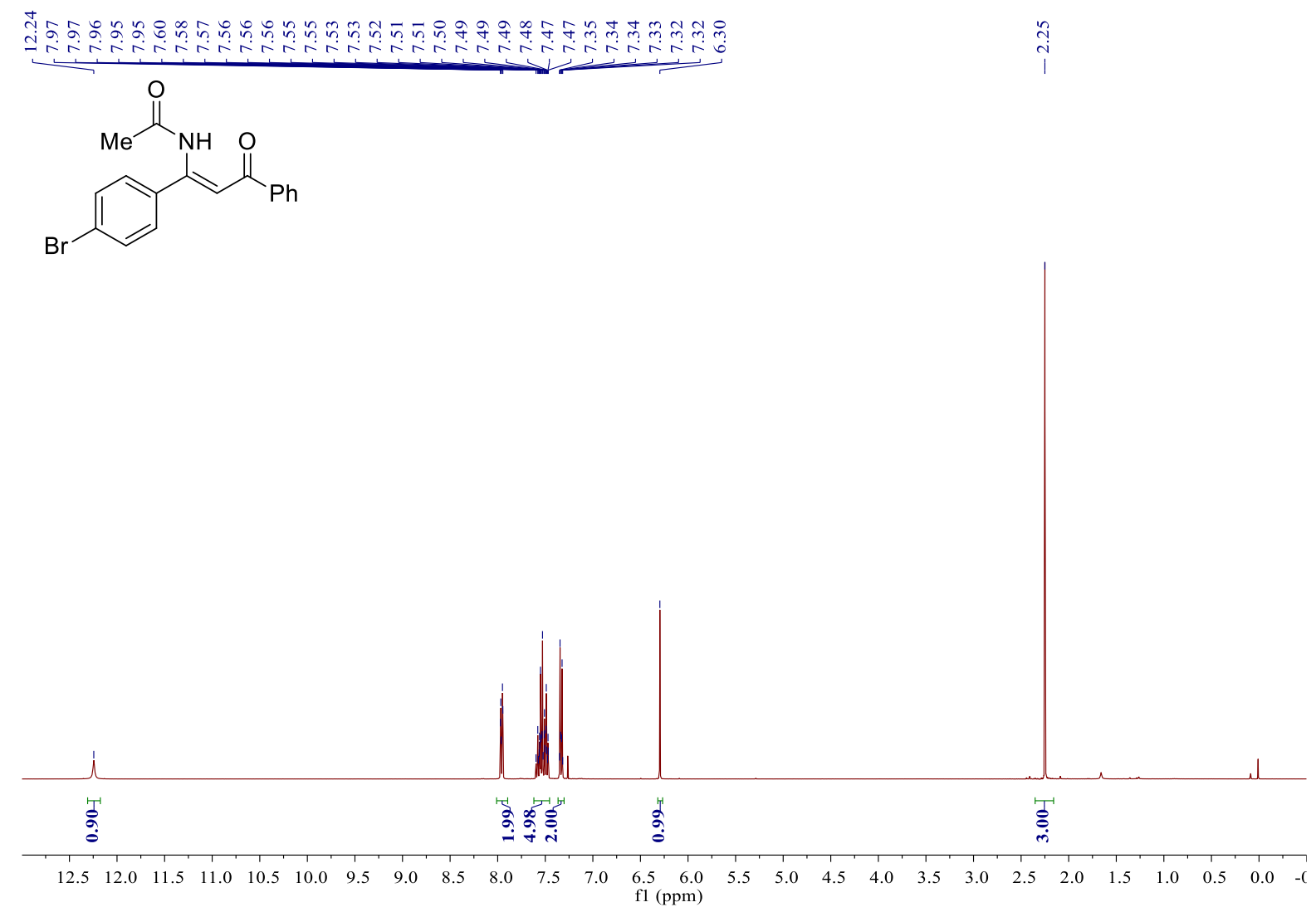

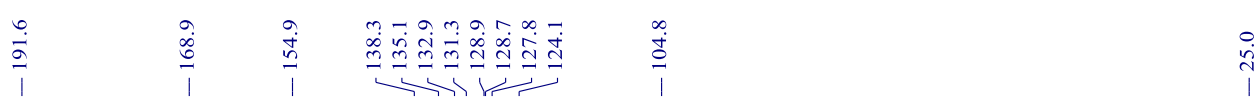

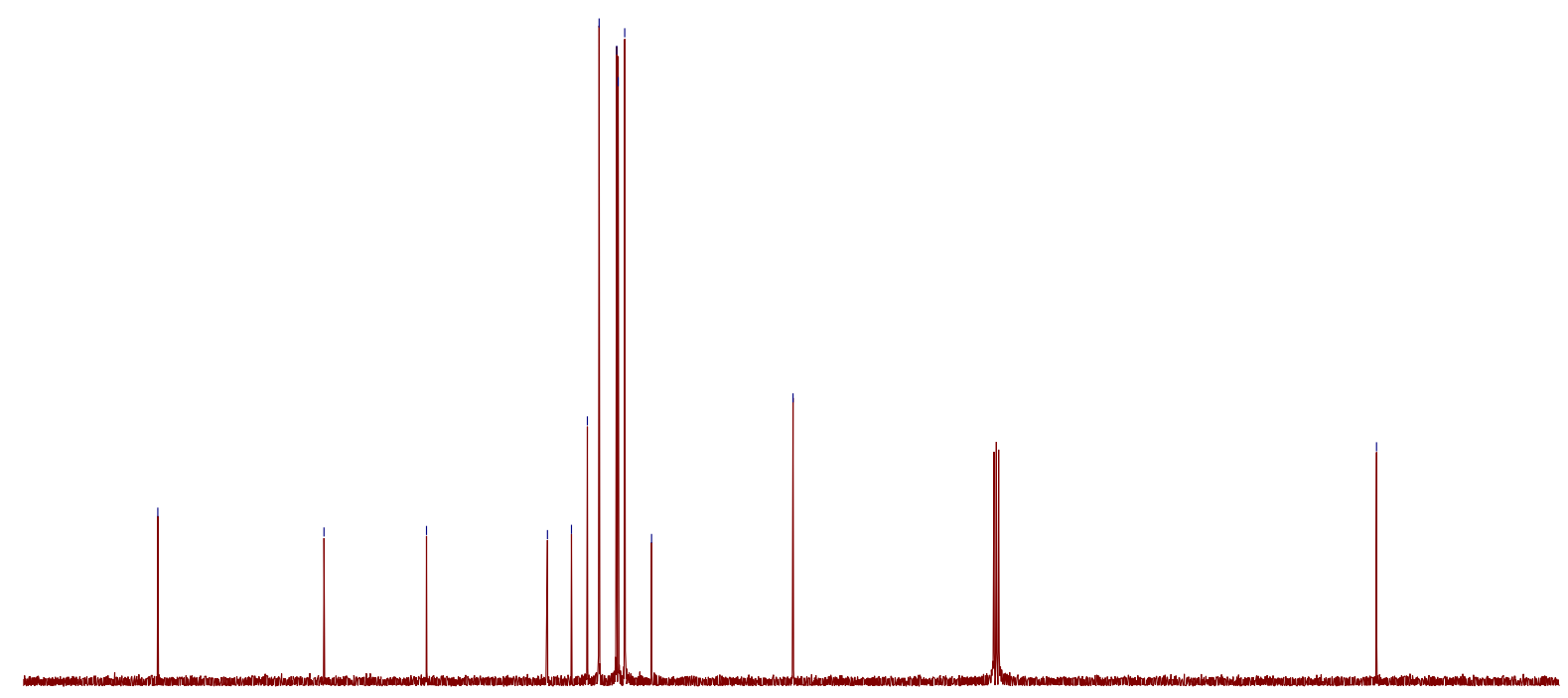

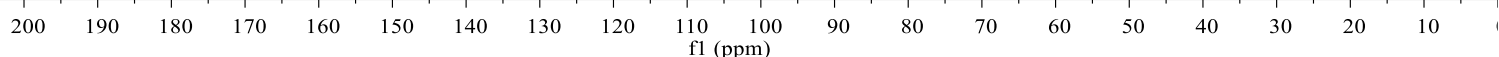

${ }^{1} \mathrm{H}$ and ${ }^{13} \mathrm{C}$ NMR spectra for product $4 \mathrm{c}\left(\mathrm{CDCl}_{3}\right)$ 

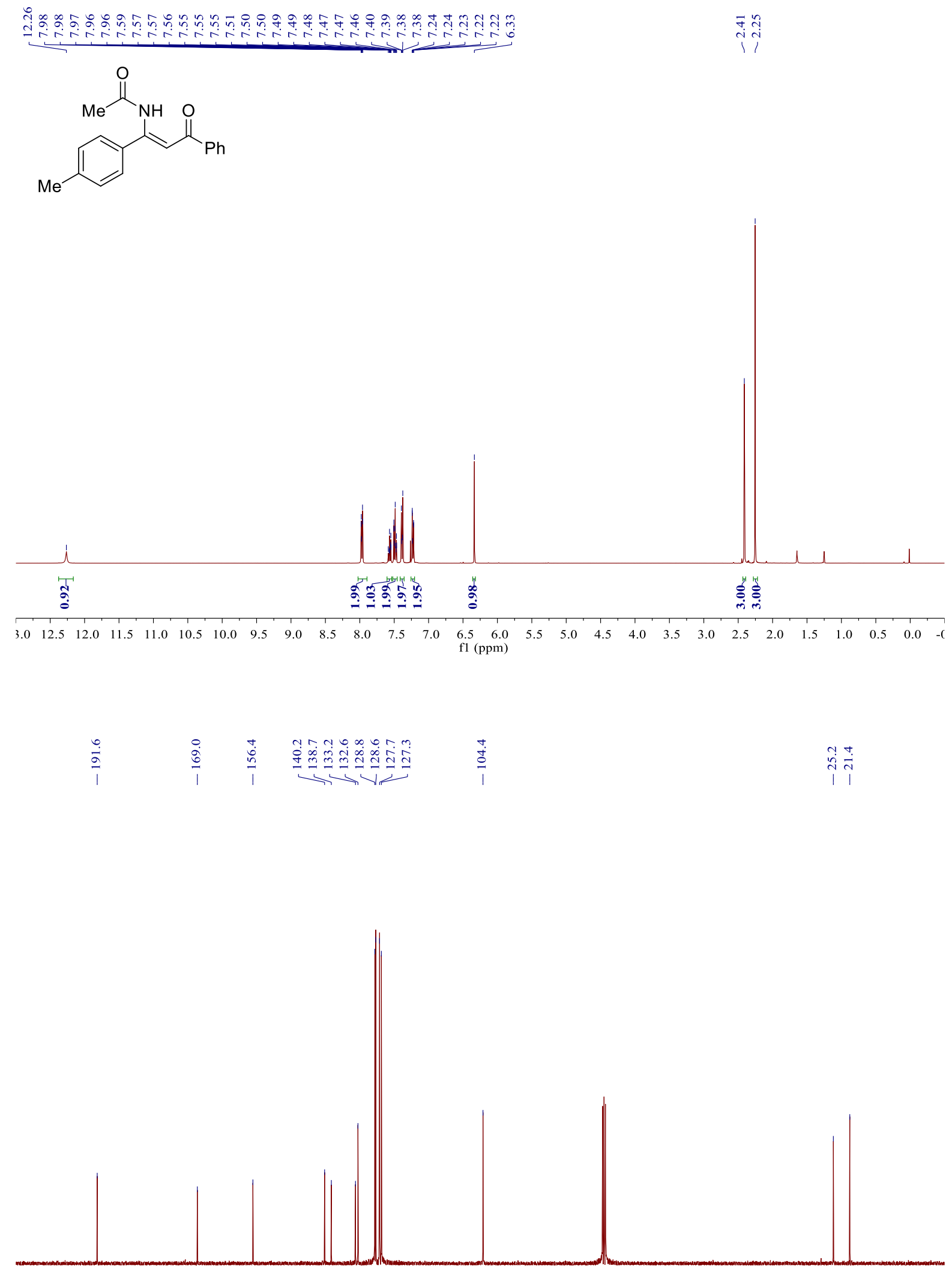

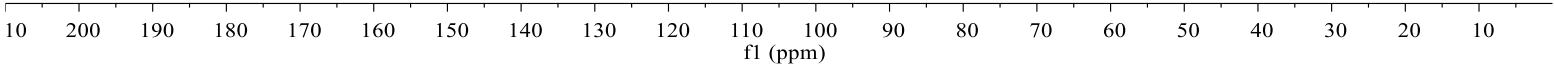

${ }^{1} \mathrm{H}$ and ${ }^{13} \mathrm{C} \mathrm{NMR}$ spectra for product $4 \mathrm{~d}\left(\mathrm{CDCl}_{3}\right)$ 

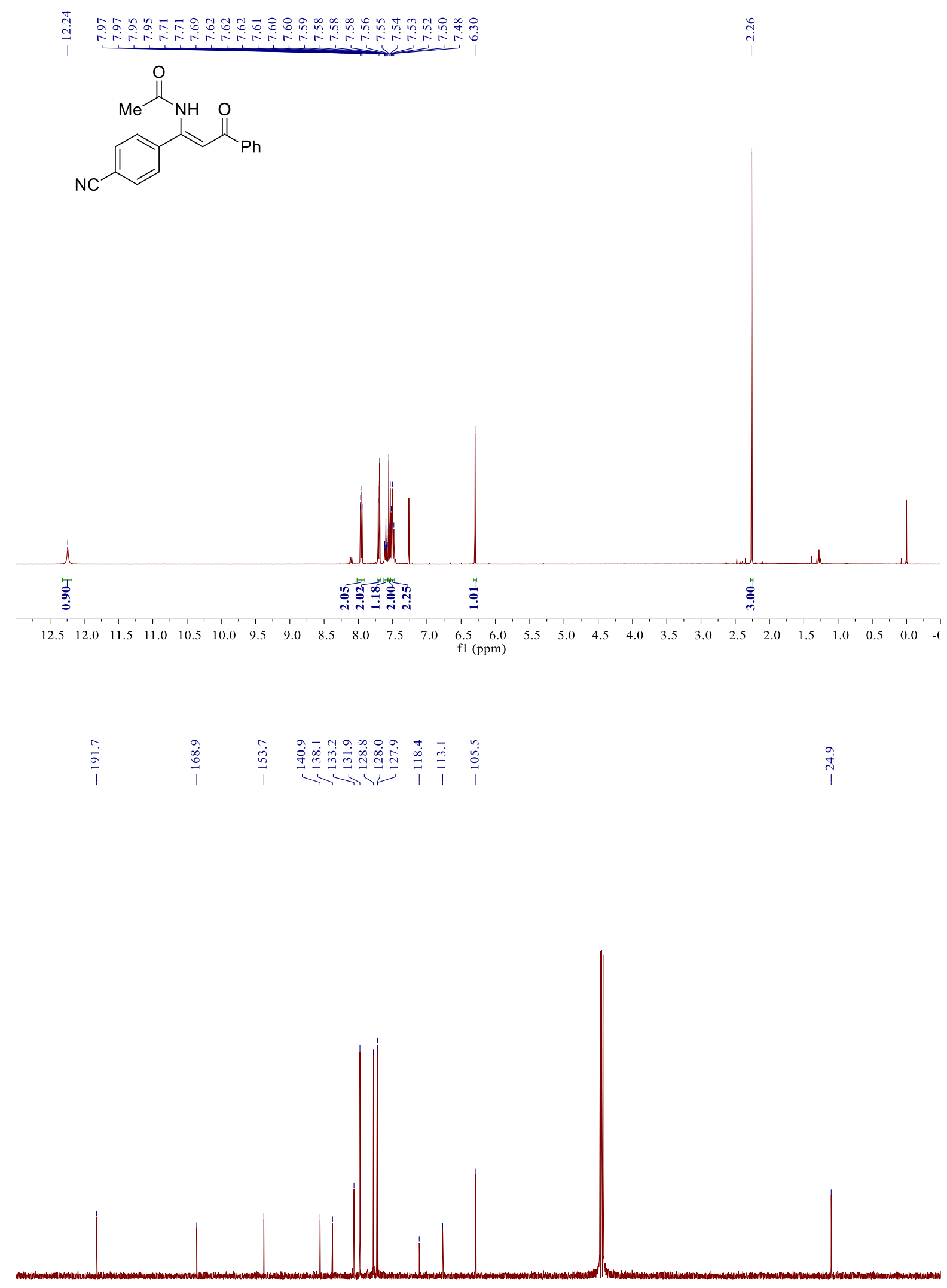

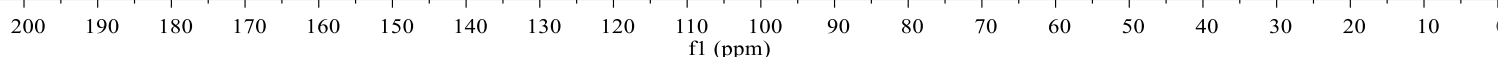

${ }^{1} \mathrm{H}$ and ${ }^{13} \mathrm{C}$ NMR spectra for product $4 \mathrm{e}\left(\mathrm{CDCl}_{3}\right)$ 


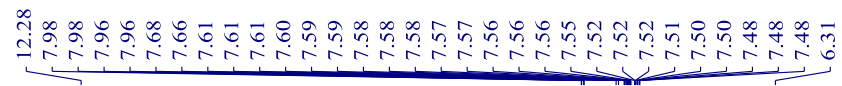<smiles>CC(=O)N/C(=C\C(=O)c1ccccc1)c1ccc(C(F)(F)F)cc1</smiles>

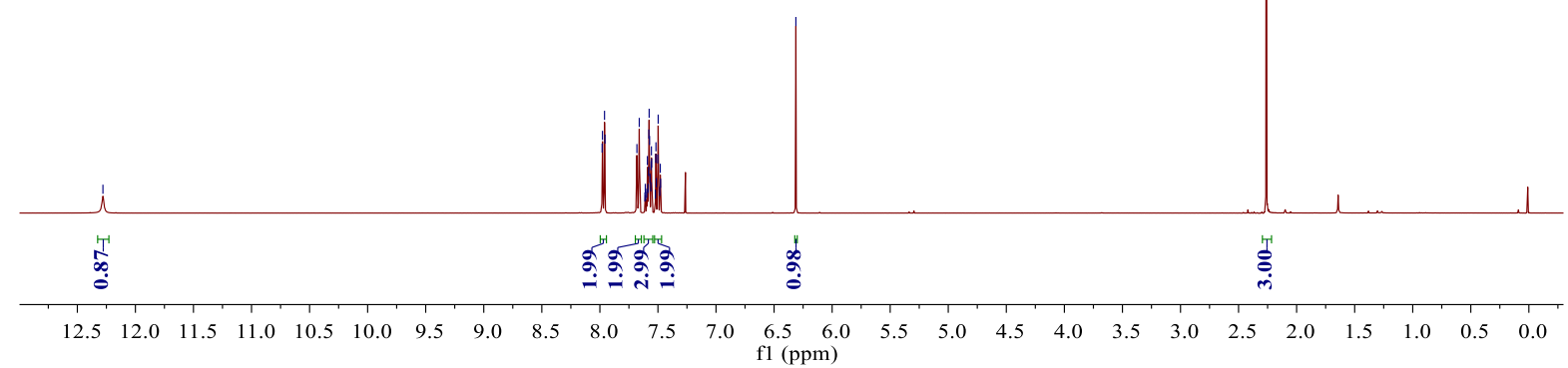

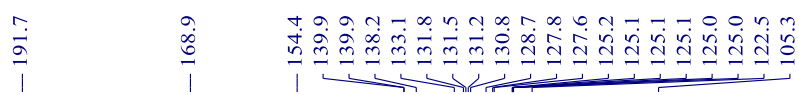

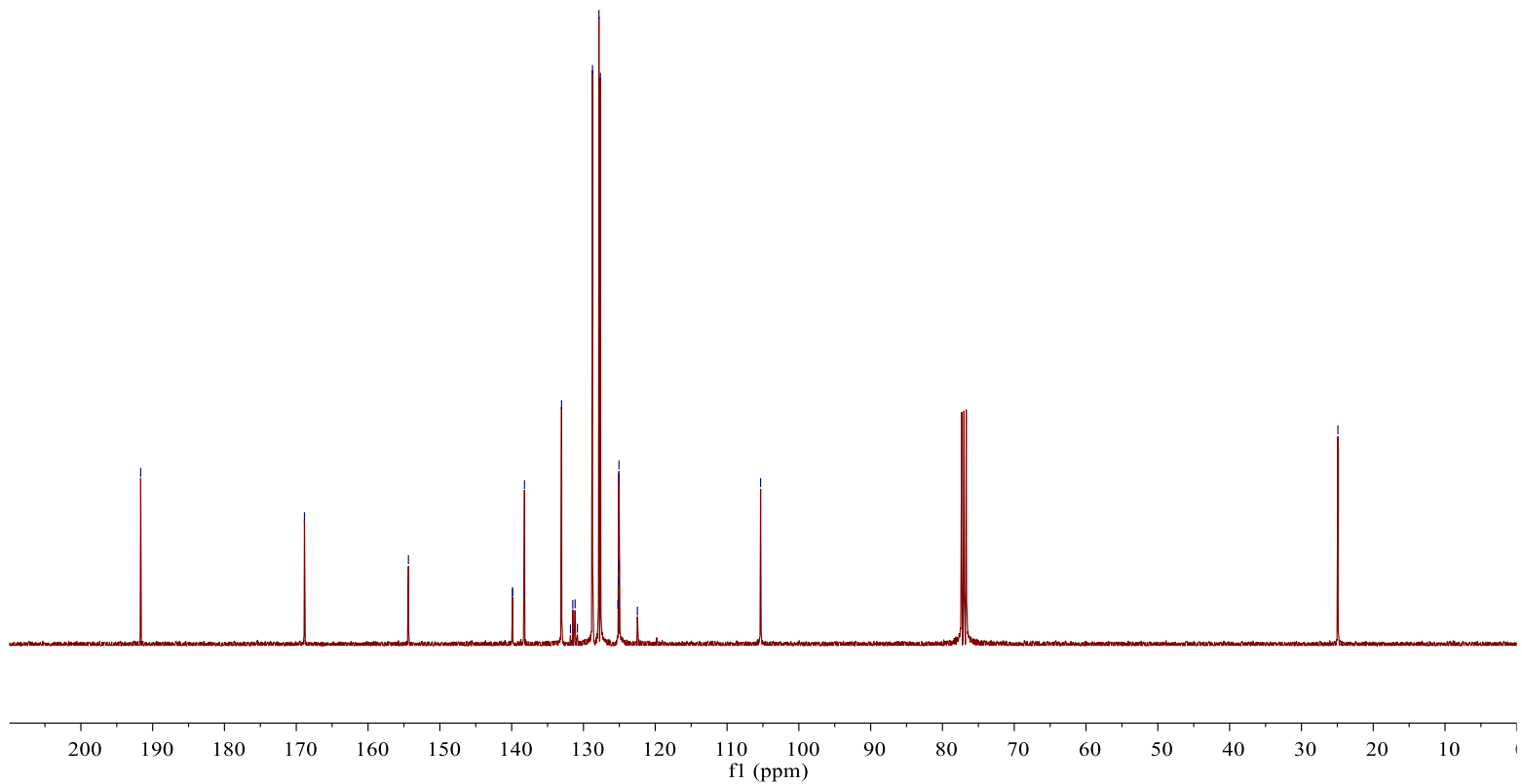




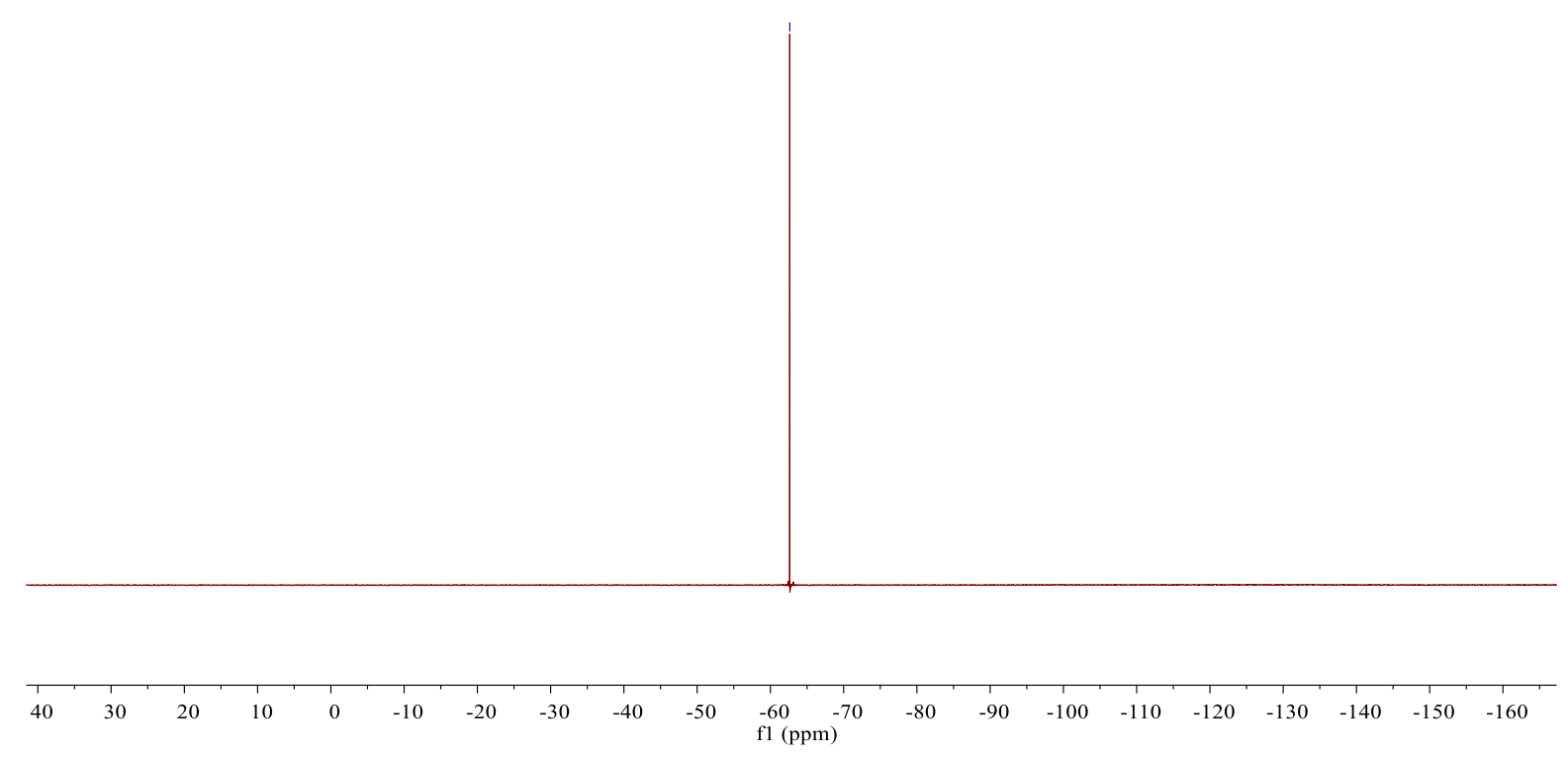

${ }^{1} \mathrm{H},{ }^{19} \mathrm{~F}$, and ${ }^{13} \mathrm{C}$ NMR spectra for product $4 \mathrm{f}\left(\mathrm{CDCl}_{3}\right)$ 


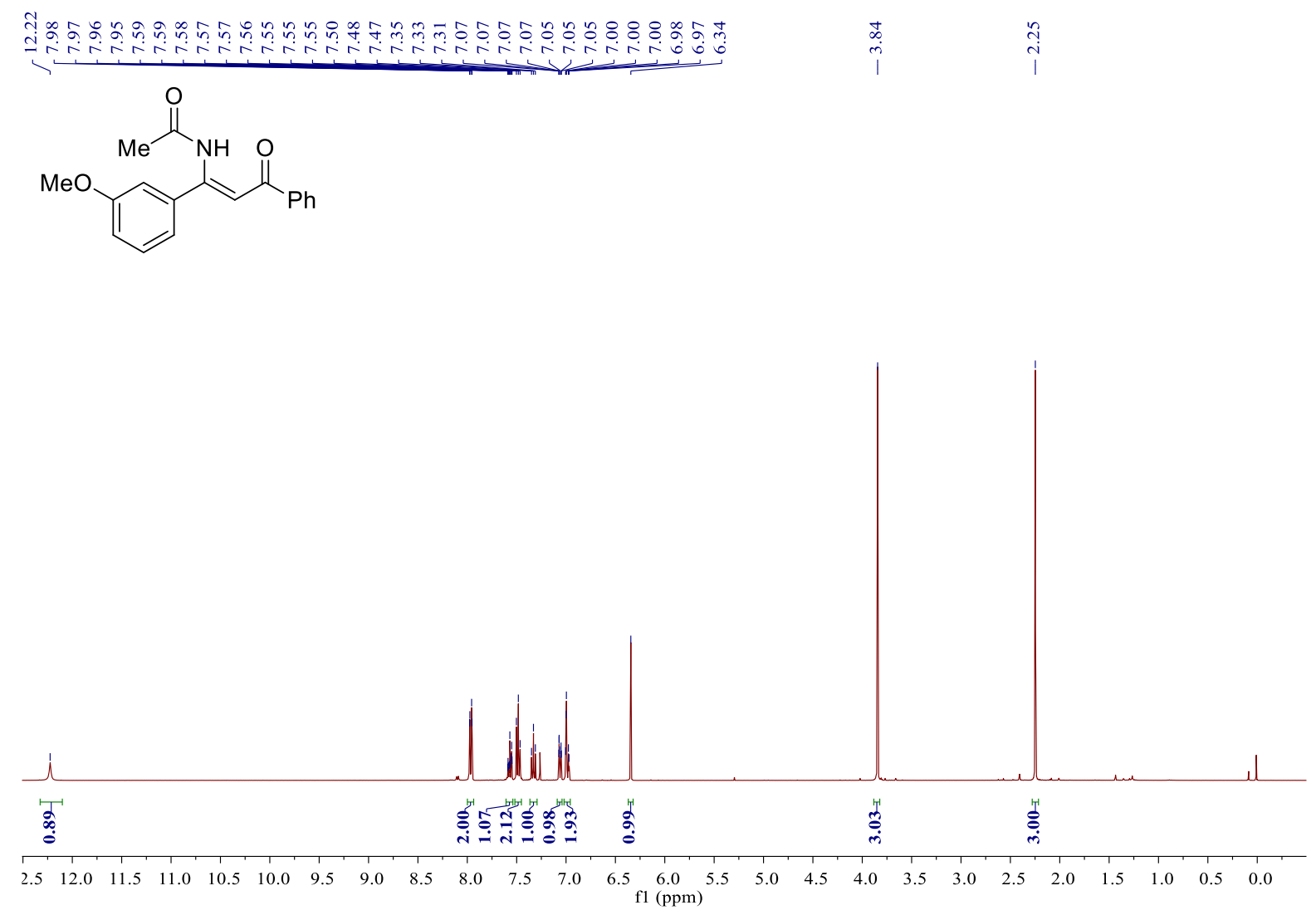

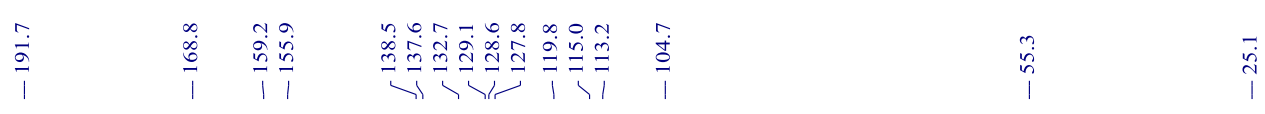

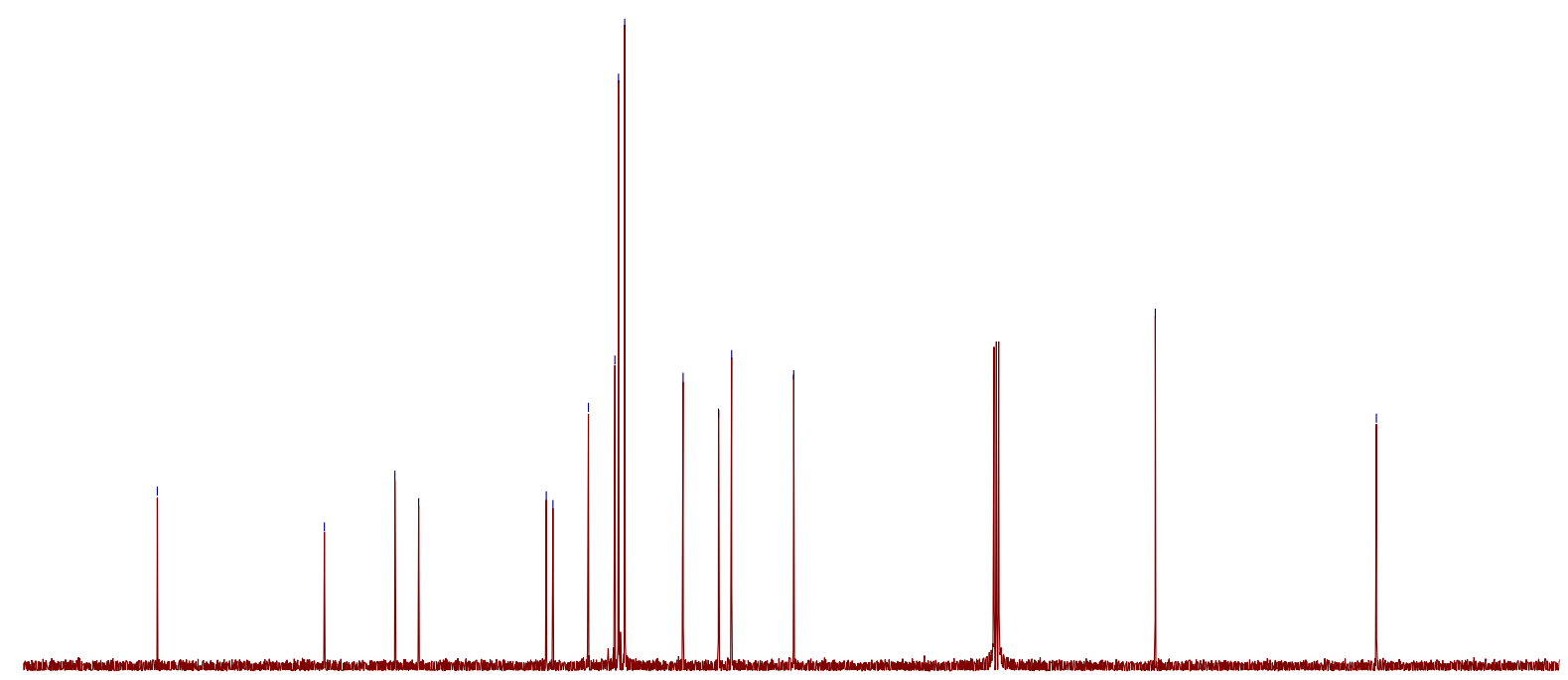

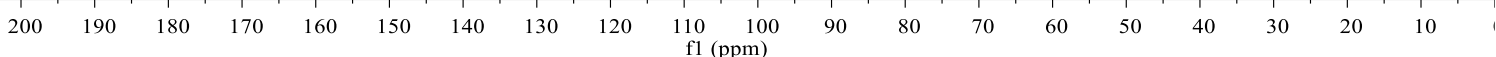

${ }^{1} \mathrm{H}$ and ${ }^{13} \mathrm{C} \mathrm{NMR}$ spectra for product $4 \mathrm{~g}\left(\mathrm{CDCl}_{3}\right)$ 


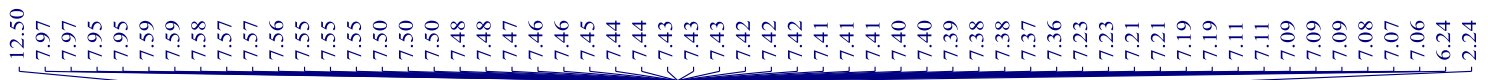<smiles>CC(=O)N/C(=C\C(=O)c1ccccc1)c1ccccc1F</smiles>

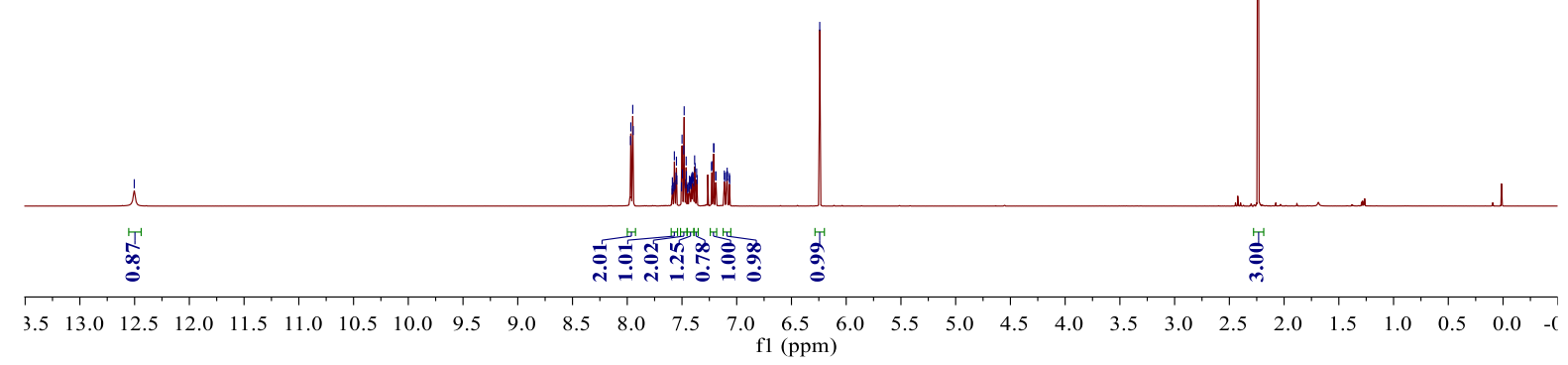

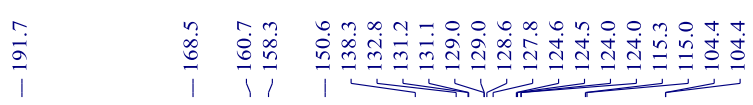

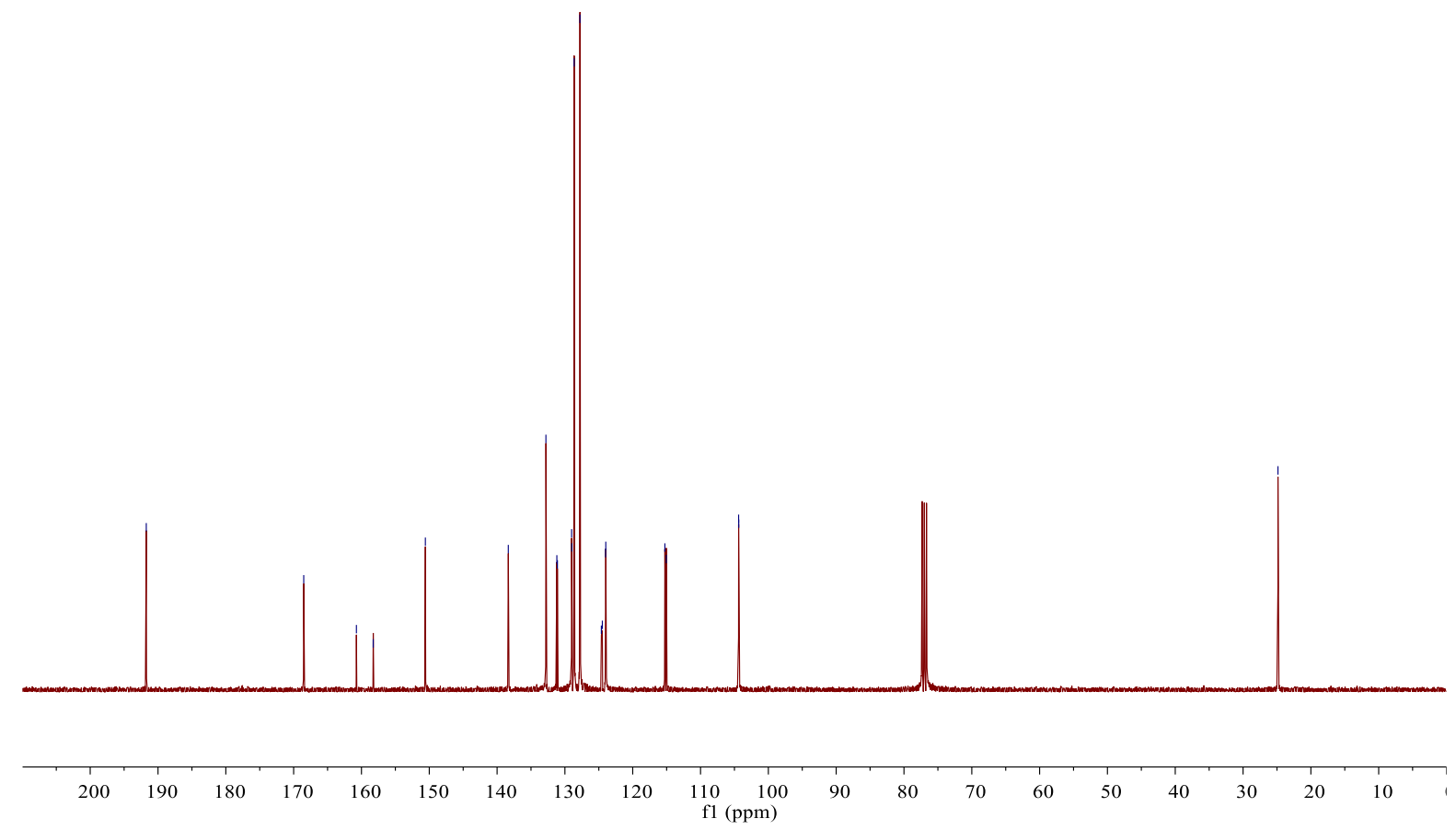




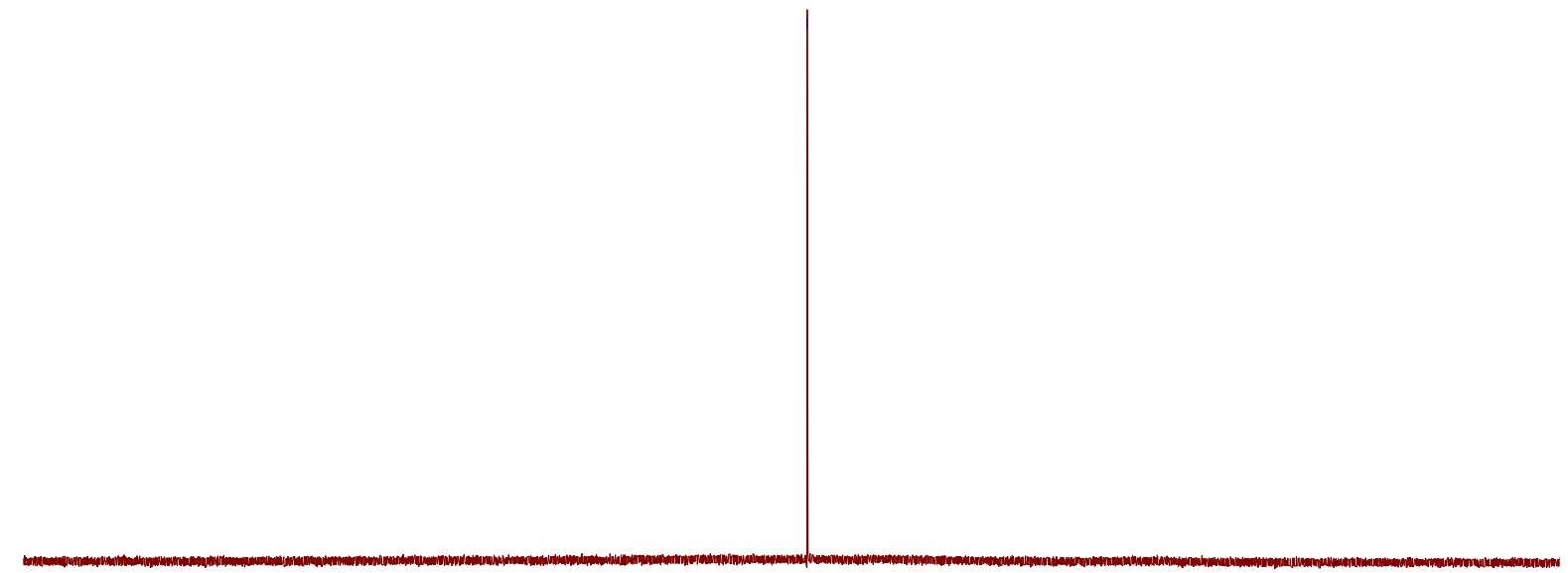

\begin{tabular}{lllllllllllllllllllllll}
\hline 0 & -10 & -20 & -30 & -40 & -50 & -60 & -70 & -80 & -90 & -100 & -110 & -120 & -130 & -140 & -150 & -160 & -170 & -180 & -190 & -200 & -210 & -220
\end{tabular}

${ }^{1} \mathrm{H},{ }^{19} \mathrm{~F}$, and ${ }^{13} \mathrm{C}$ NMR spectra for product $4 \mathrm{~h}\left(\mathrm{CDCl}_{3}\right)$ 


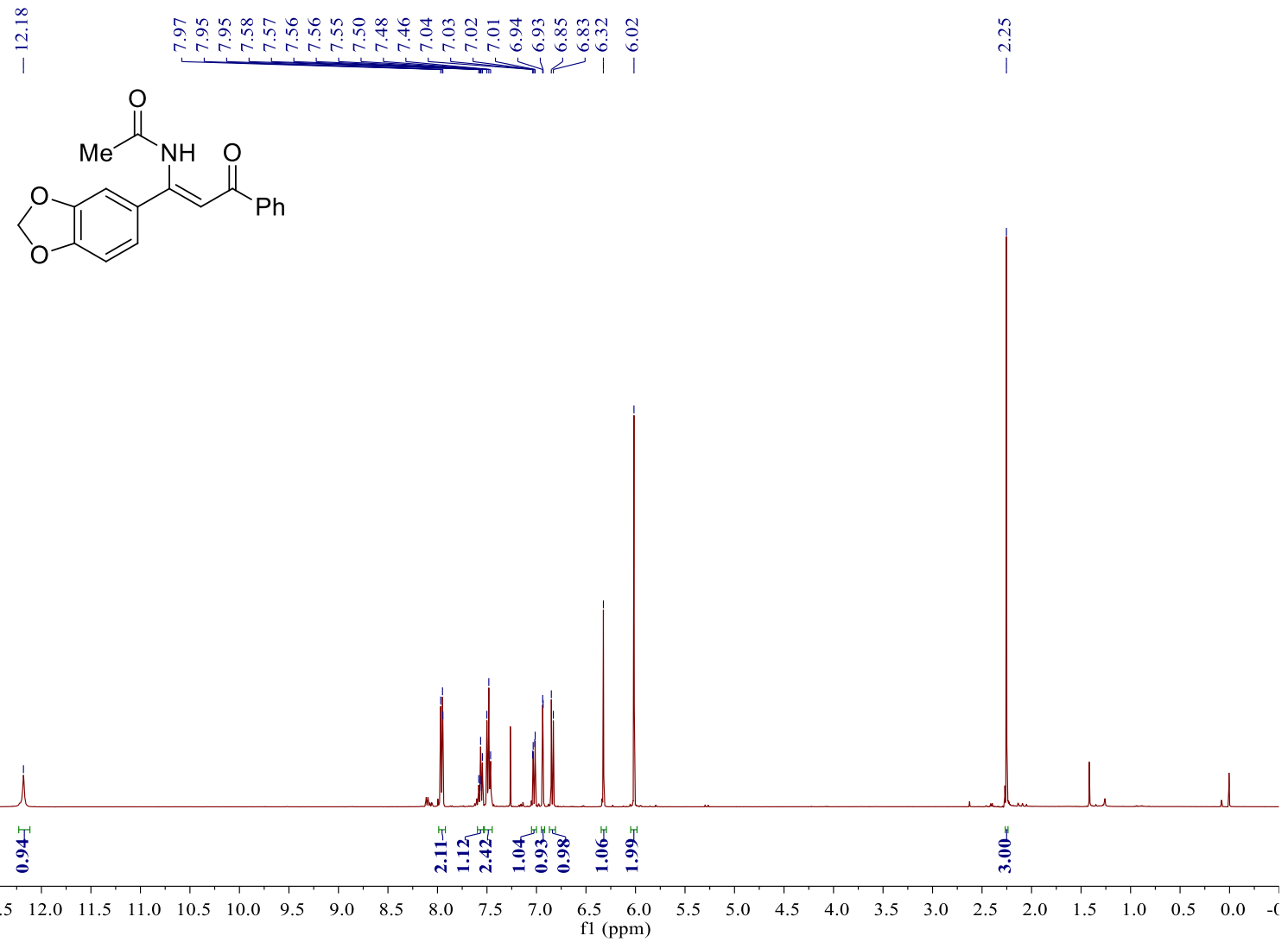

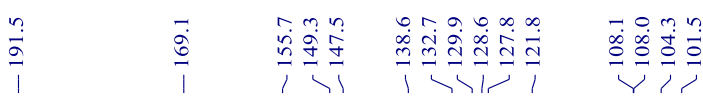

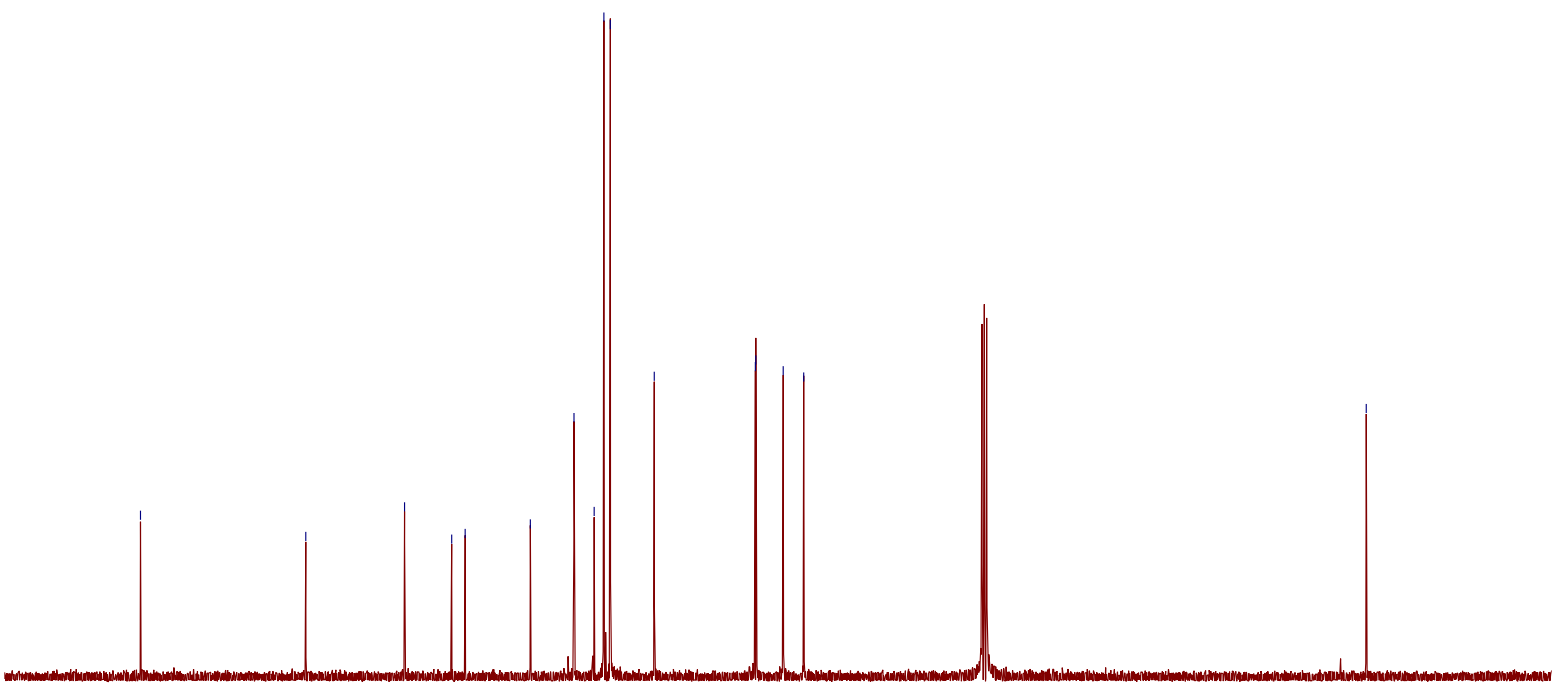

$\begin{array}{lllllllllll}200 & 190 & 180 & 170 & 160 & 150 & 140 & 130 & 120 & 110 & 100 \\ & & & & & & & & \end{array}$

${ }^{1} \mathrm{H}$ and ${ }^{13} \mathrm{C}$ NMR spectra for product $4 \mathrm{i}\left(\mathrm{CDCl}_{3}\right)$ 


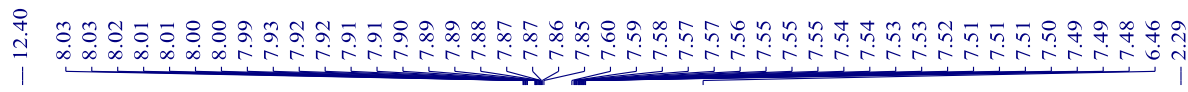<smiles>CC(=O)N/C(=C\C(=O)c1ccccc1)c1ccc2ccccc2c1</smiles>

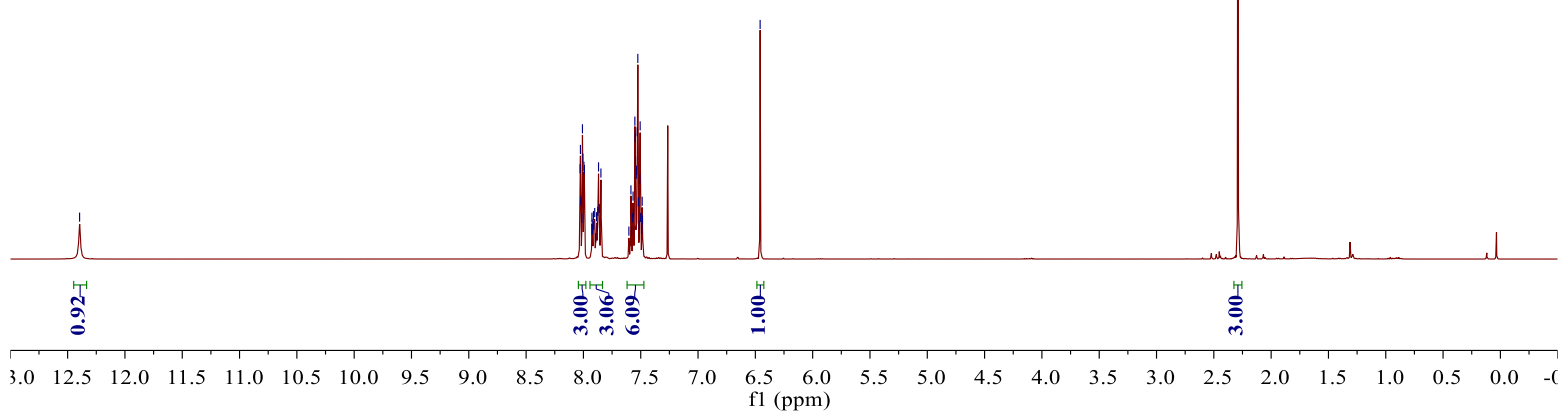

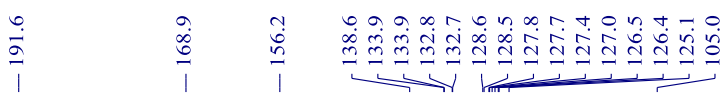

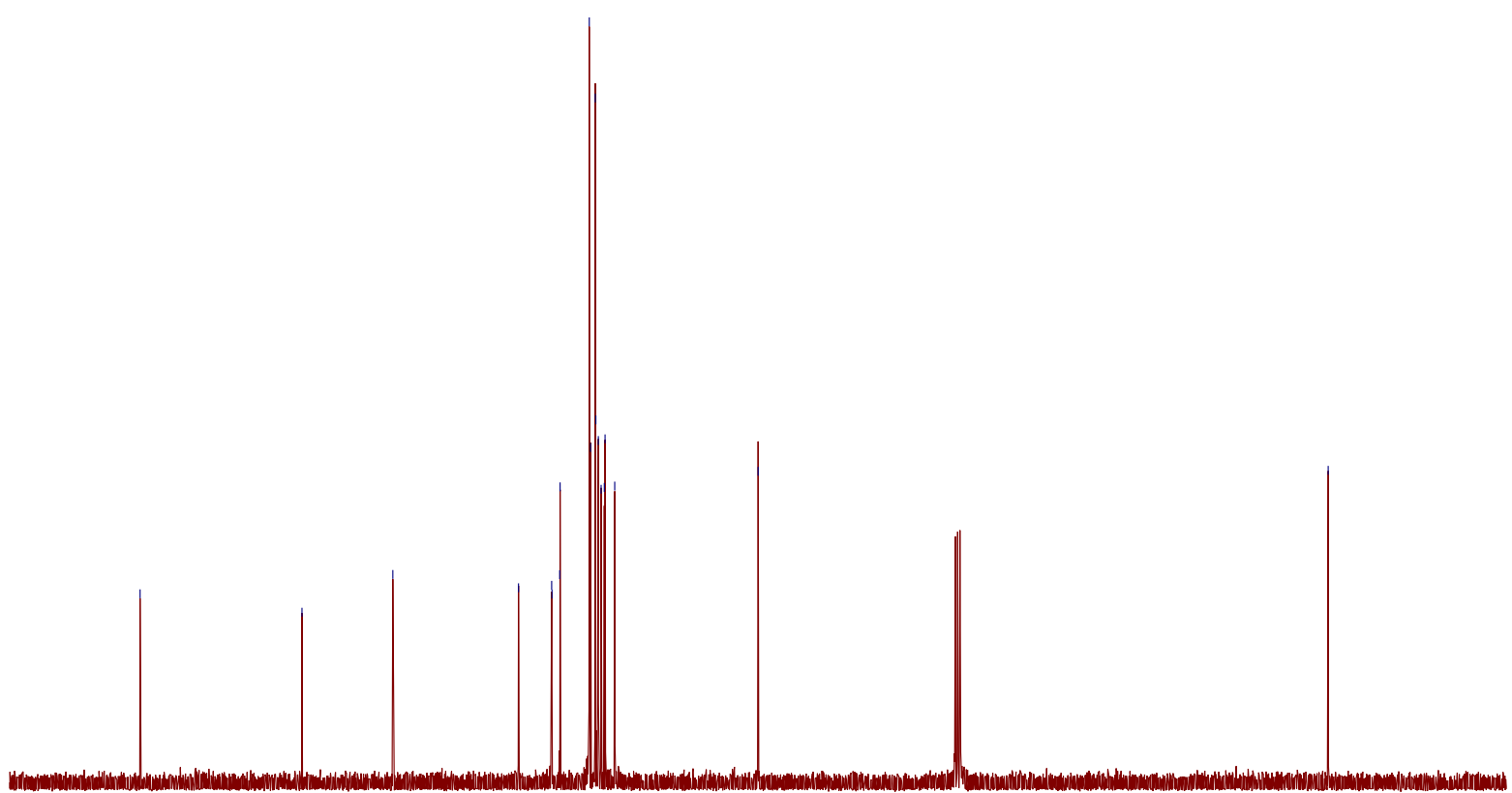

$\begin{array}{lllllllllll}200 & 190 & 180 & 170 & 160 & 150 & 140 & 130 & 120 & 110 \quad \begin{array}{l}100 \\ \text { f1 (ppm) }\end{array}\end{array}$

${ }^{1} \mathrm{H}$ and ${ }^{13} \mathrm{C}$ NMR spectra for product $4 \mathrm{j}\left(\mathrm{CDCl}_{3}\right)$ 


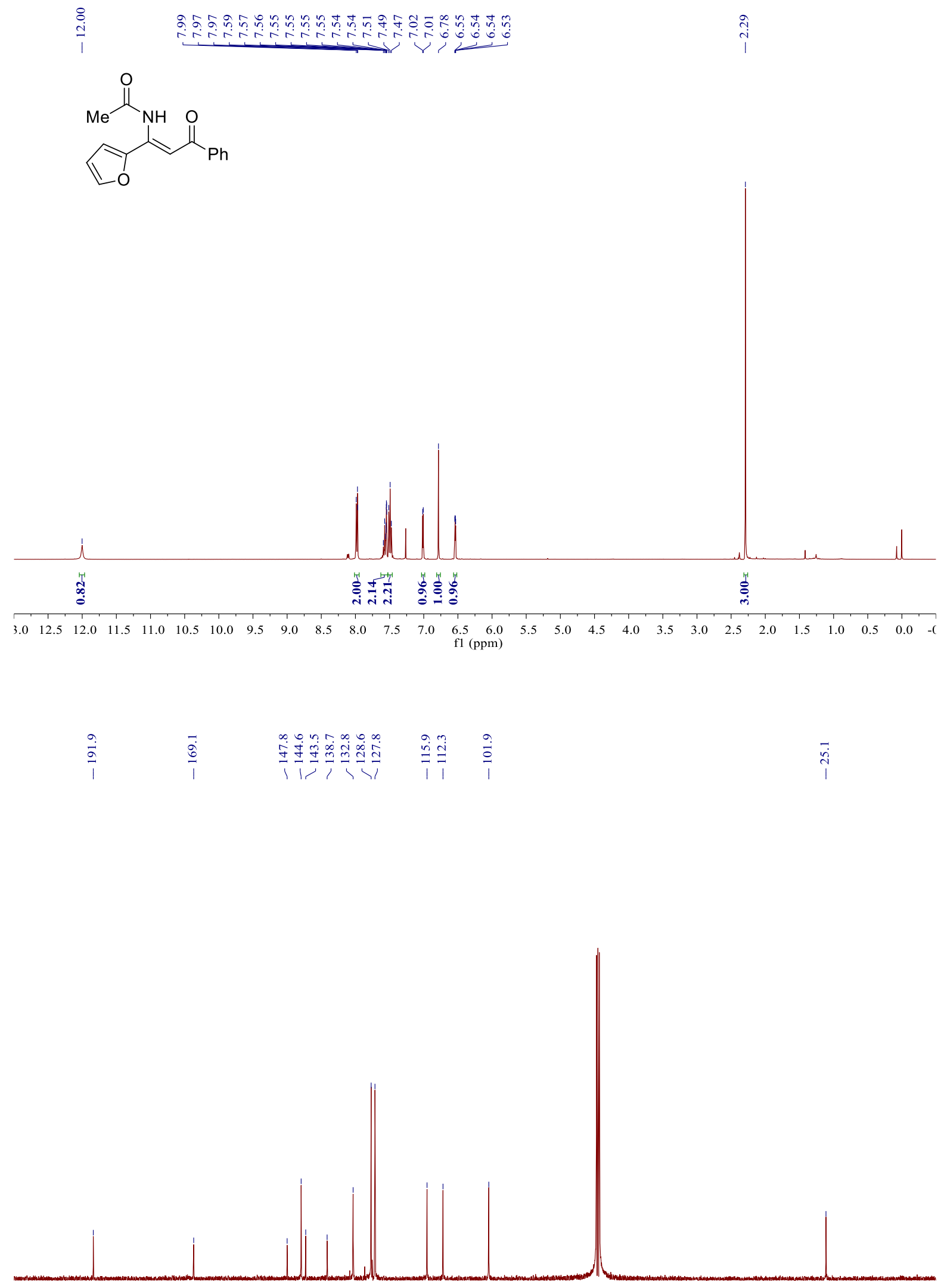

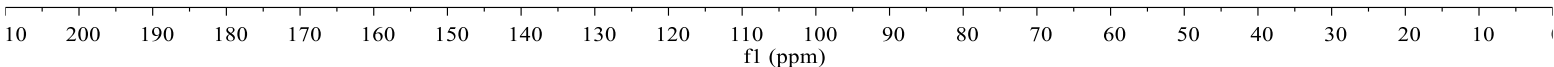

${ }^{1} \mathrm{H}$ and ${ }^{13} \mathrm{C}$ NMR spectra for product $4 \mathrm{k}\left(\mathrm{CDCl}_{3}\right)$ 

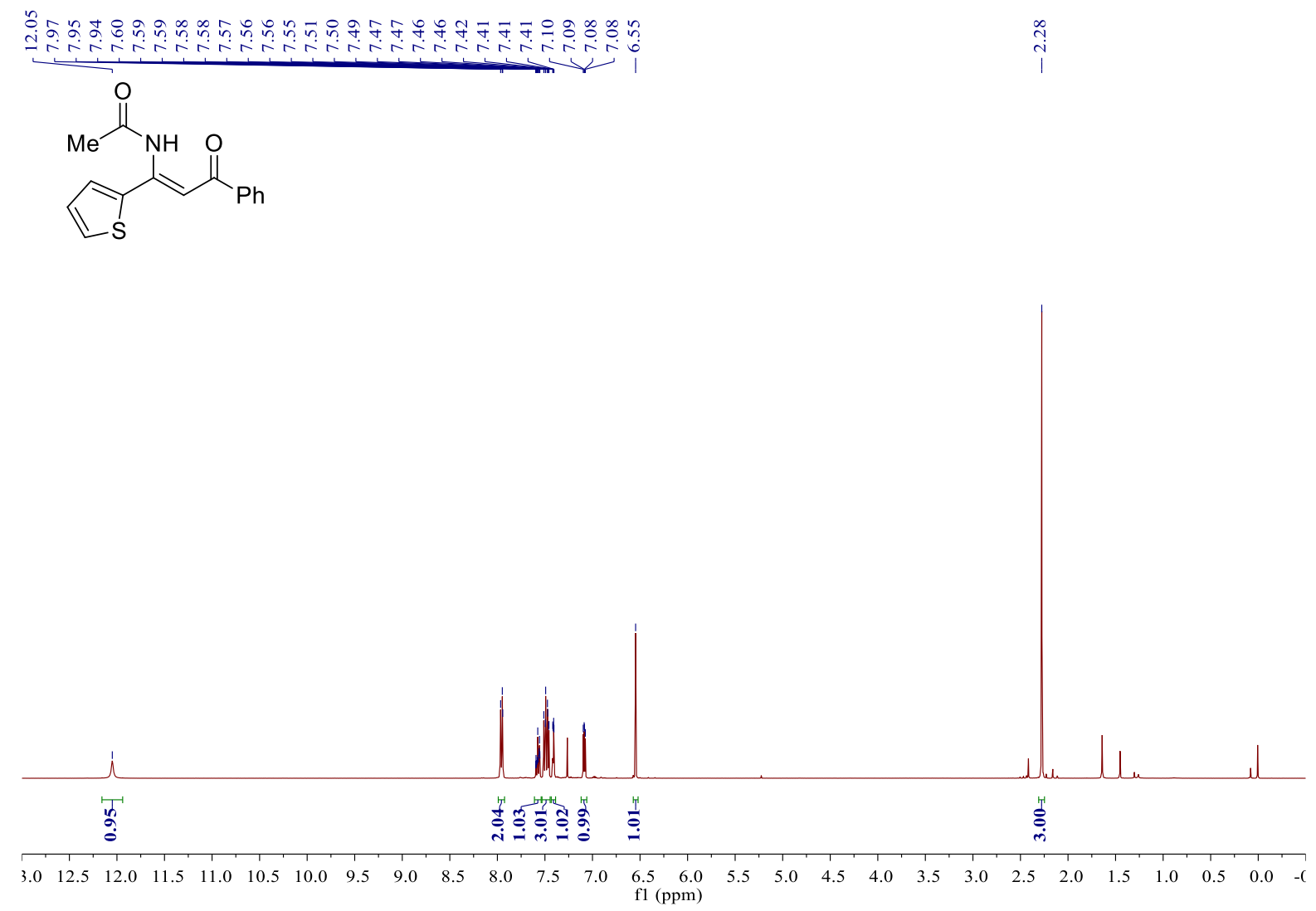

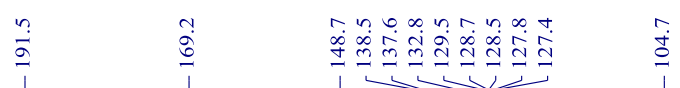

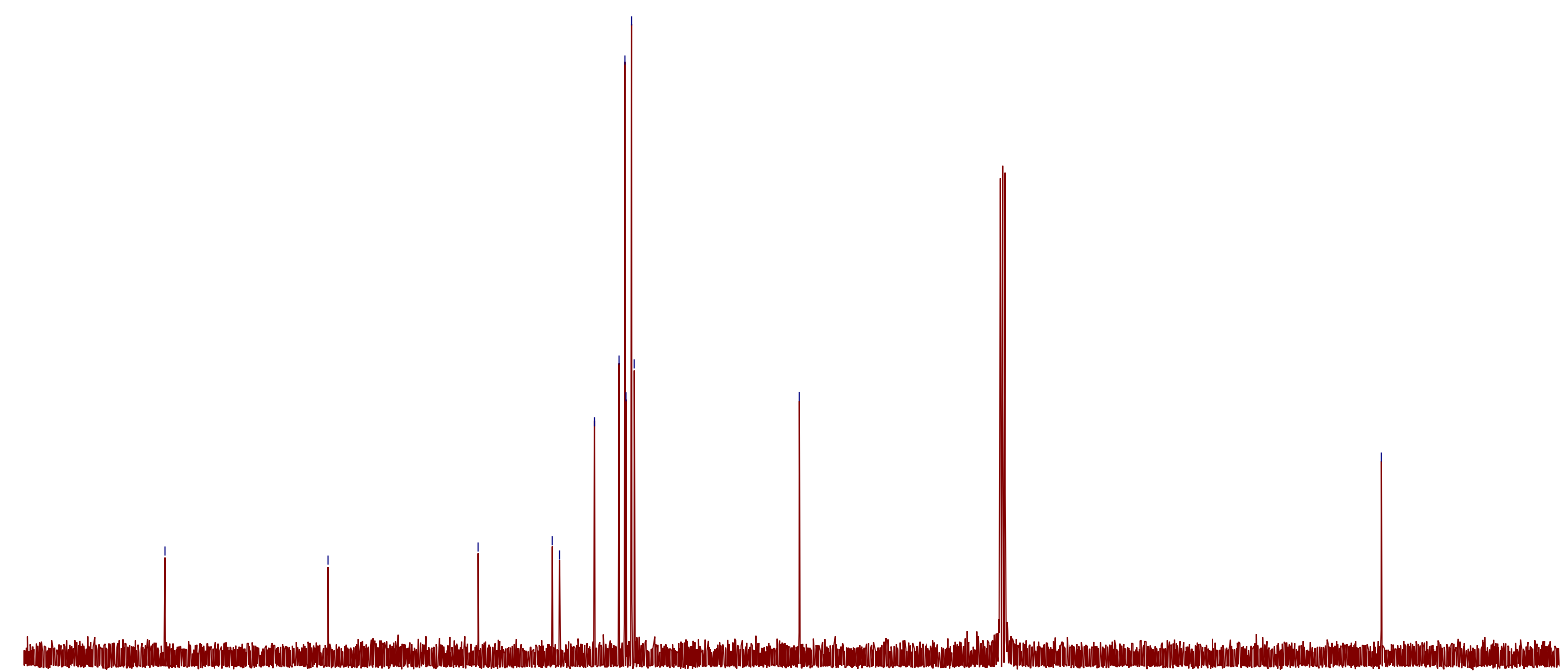

$\begin{array}{lllllllllllllllllllll}110 & 200 & 190 & 180 & 170 & 160 & 150 & 140 & 130 & 120 & \begin{array}{c}110 \\ \mathrm{f} 1(\mathrm{ppm})\end{array} & 90 & 80 & 70 & 60 & 50 & 40 & 30 & 20 & 10\end{array}$

${ }^{1} \mathrm{H}$ and ${ }^{13} \mathrm{C}$ NMR spectra for product $4 \mathrm{I}\left(\mathrm{CDCl}_{3}\right)$ 


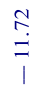<smiles>CC(=O)NC1=C(C(=O)c2ccccc2)Cc2ccccc21</smiles>

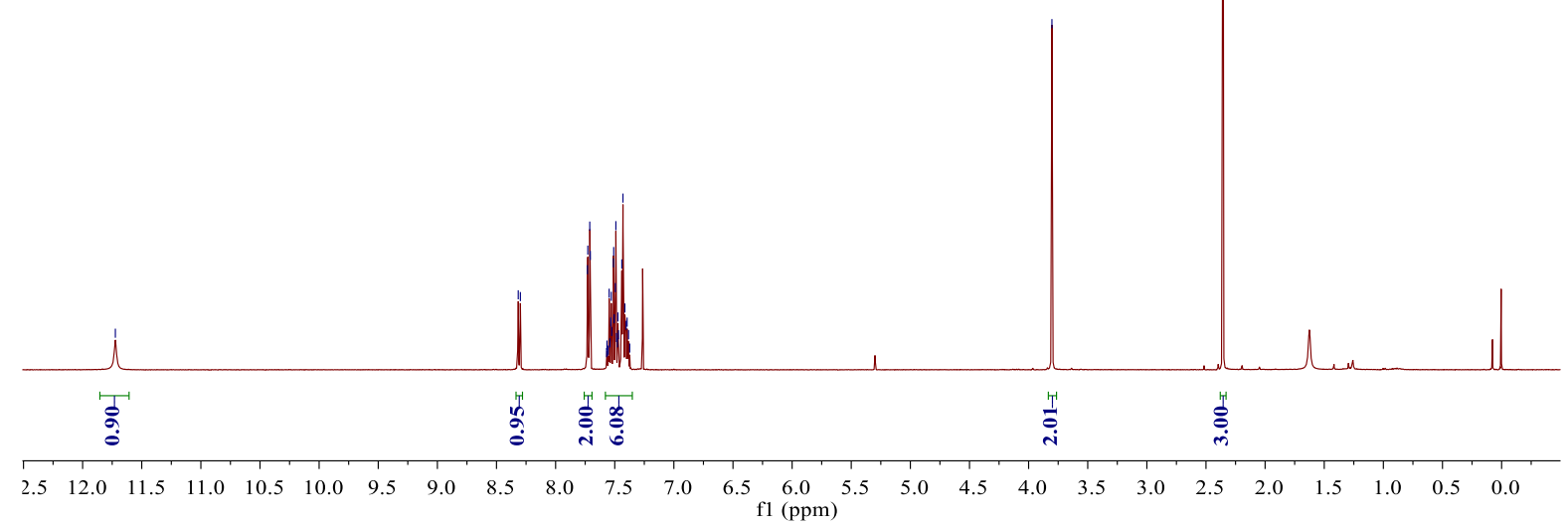

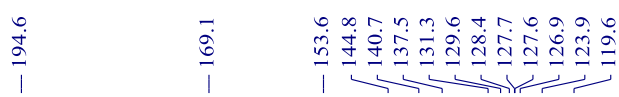

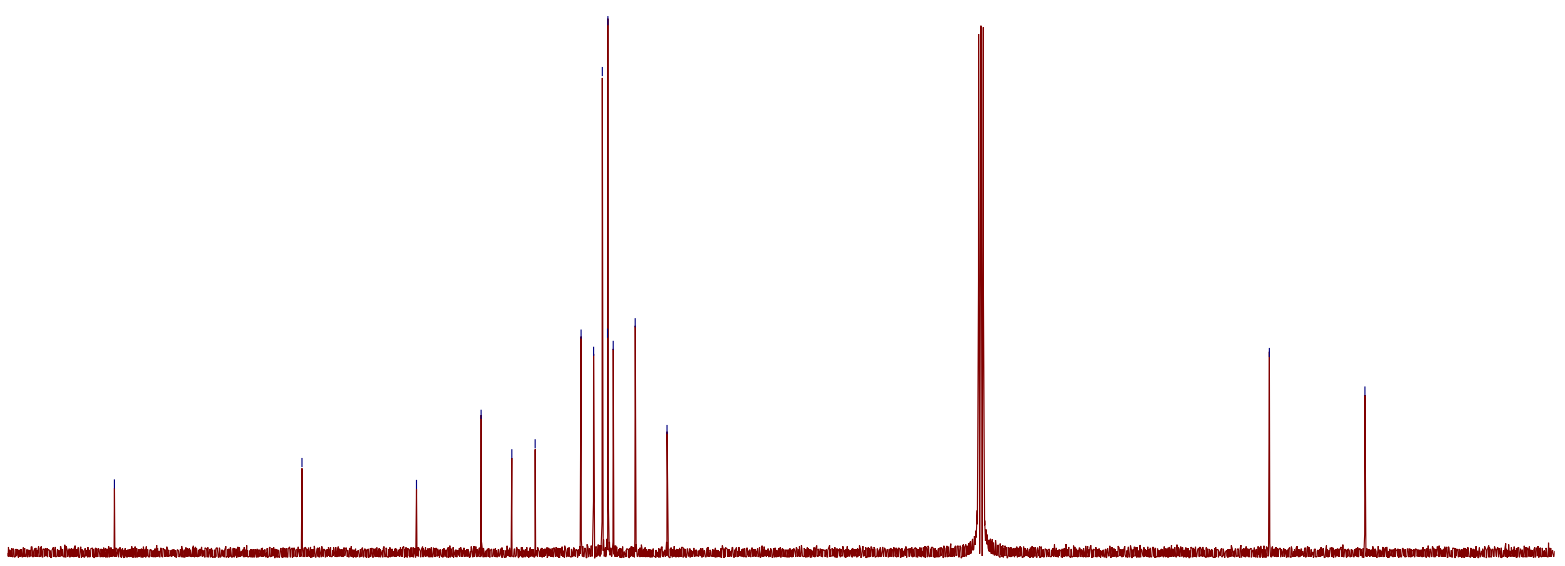

$\begin{array}{llllllllll}200 & 190 & 180 & 170 & 160 & 150 & 140 & 130 & 120 & \begin{array}{c}110 \\ \mathrm{f} 1(\mathrm{ppm})\end{array}\end{array}$

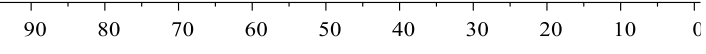

${ }^{1} \mathrm{H}$ and ${ }^{13} \mathrm{C}$ NMR spectra for product $4 \mathrm{~m}\left(\mathrm{CDCl}_{3}\right)$ 


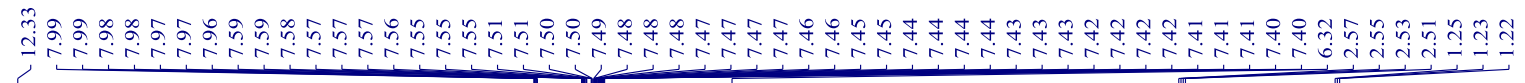

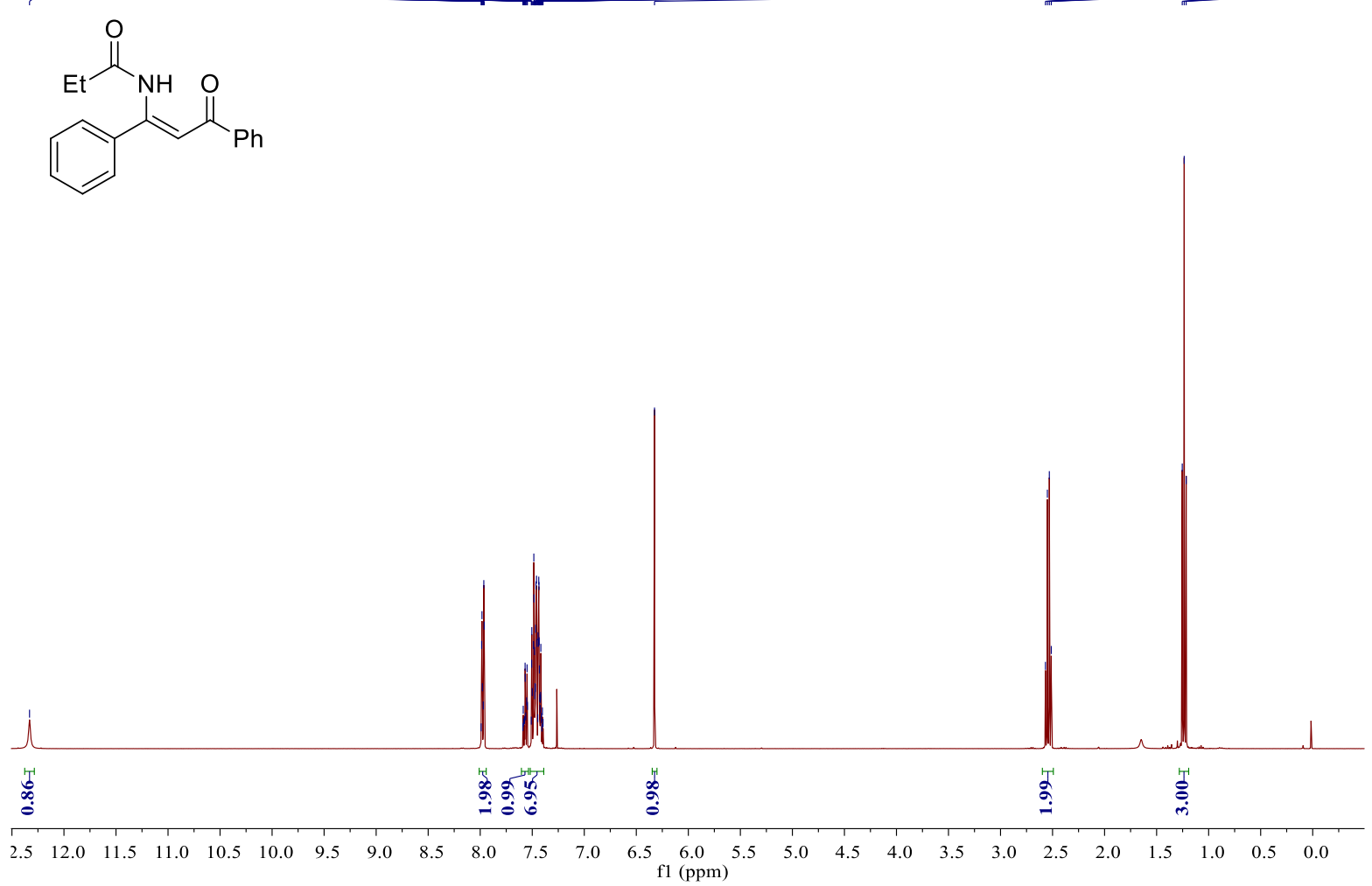

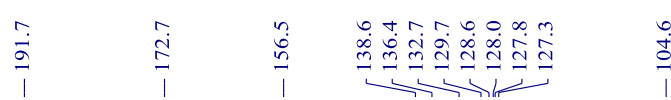
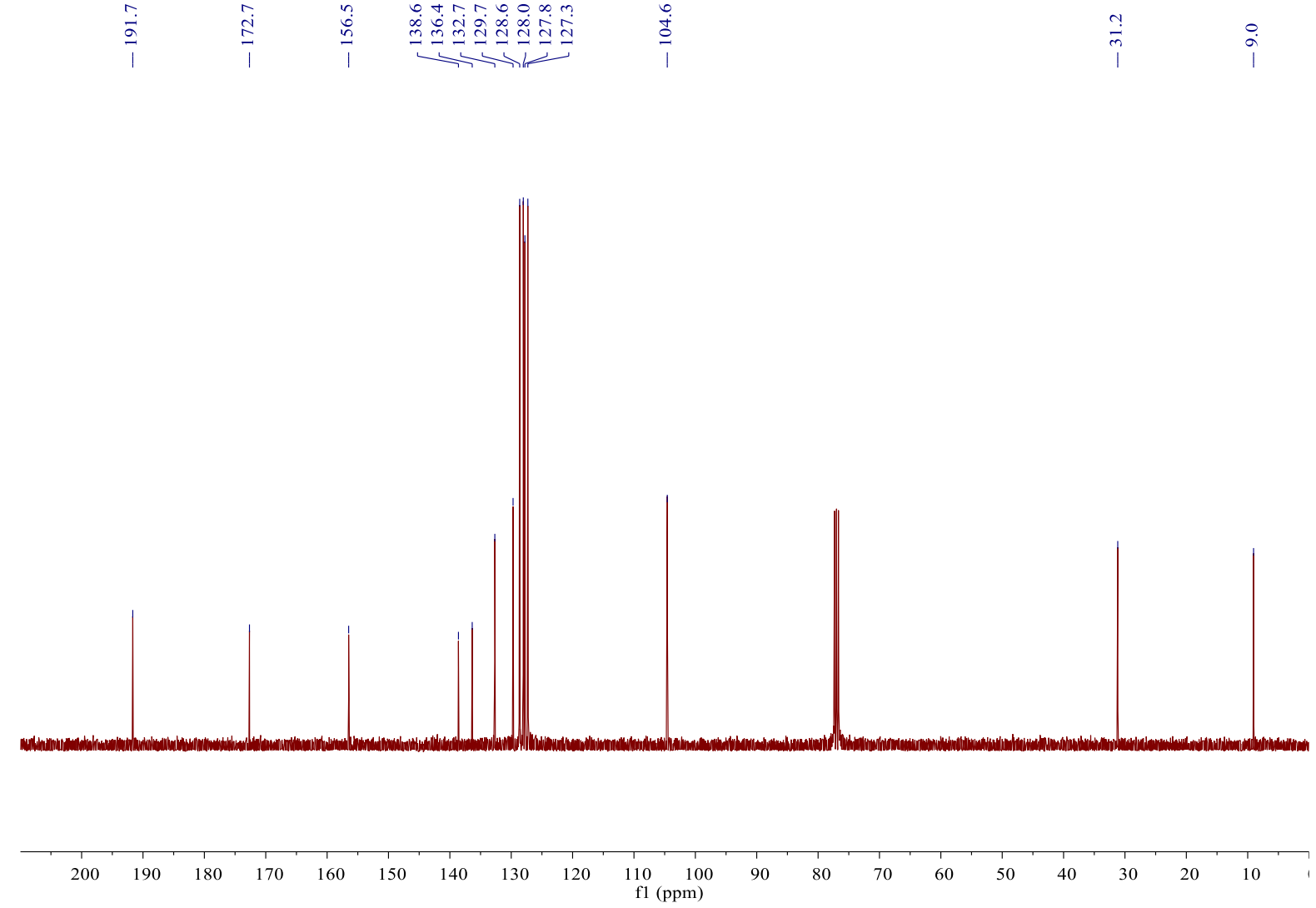

${ }^{1} \mathrm{H}$ and ${ }^{13} \mathrm{C}$ NMR spectra for product $4 \mathrm{n}\left(\mathrm{CDCl}_{3}\right)$ 


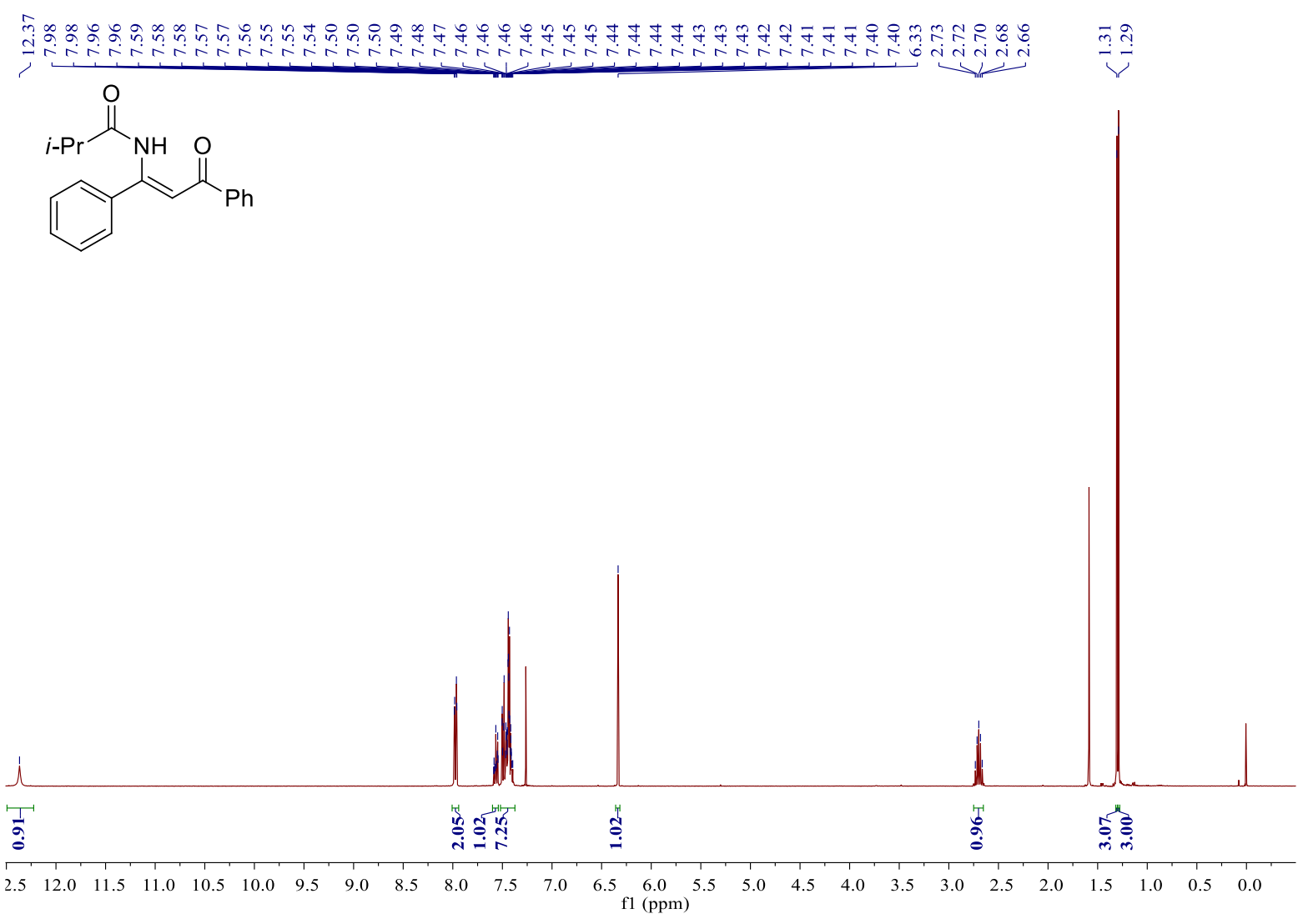

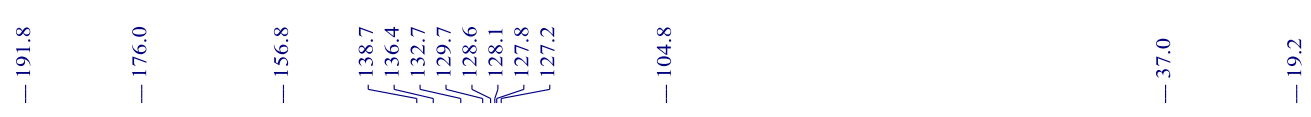

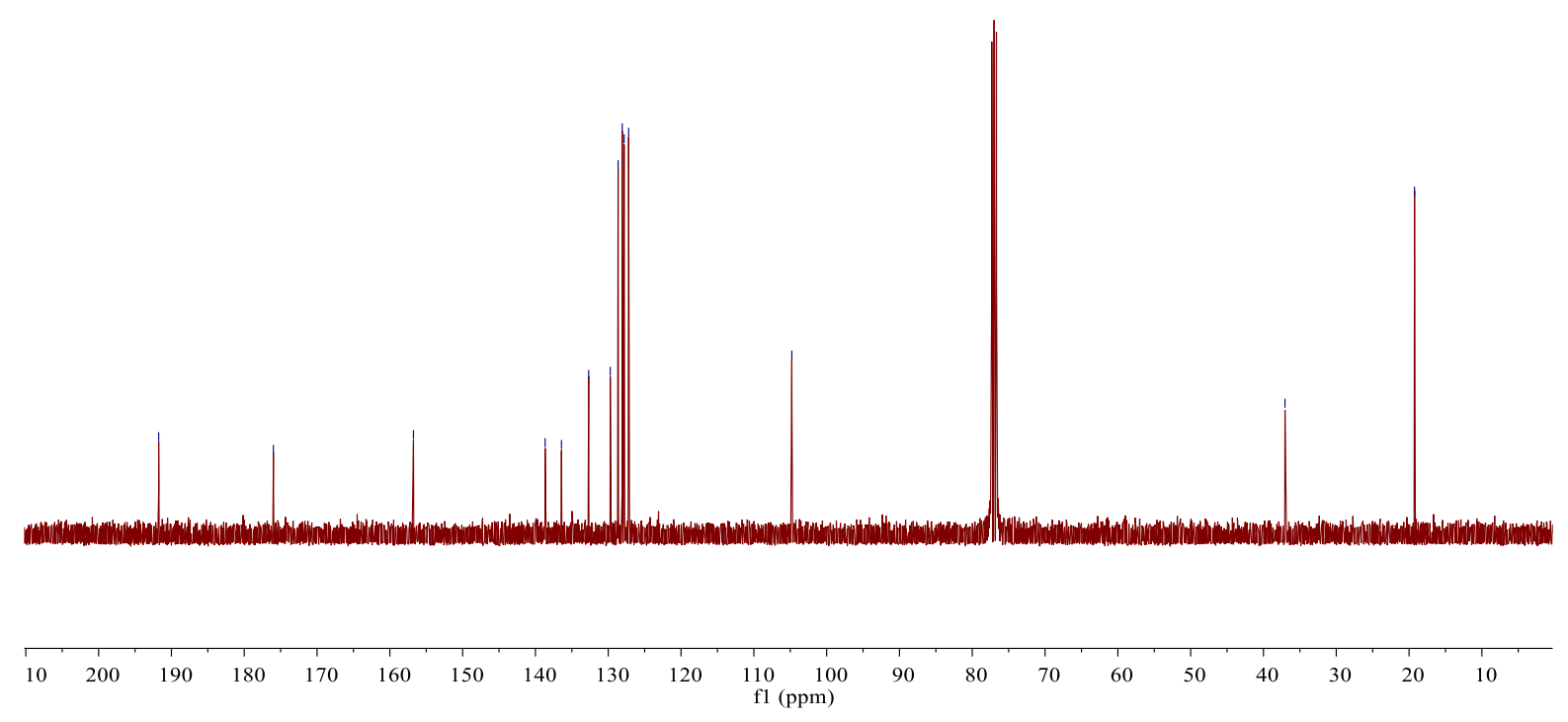

${ }^{1} \mathrm{H}$ and ${ }^{13} \mathrm{C}$ NMR spectra for product $40\left(\mathrm{CDCl}_{3}\right)$ 
年

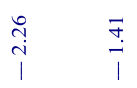<smiles>CC(=O)N/C(=C\C(=O)c1ccccc1)C(C)(C)C</smiles>

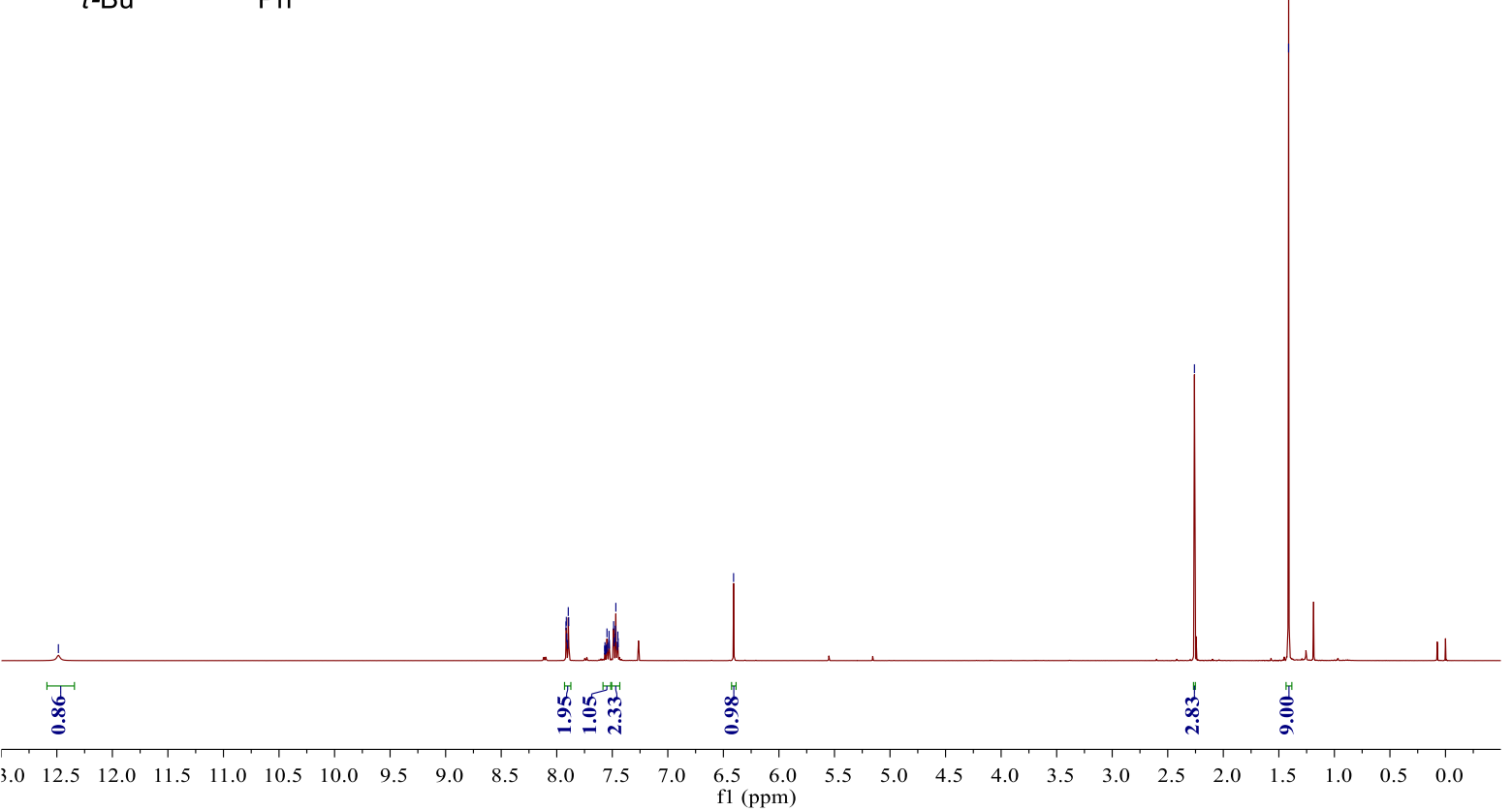

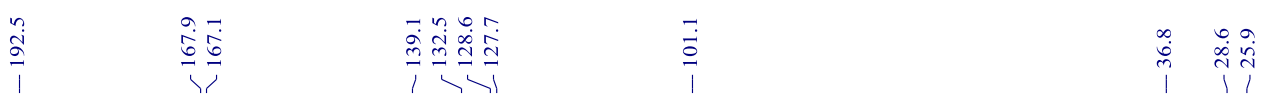

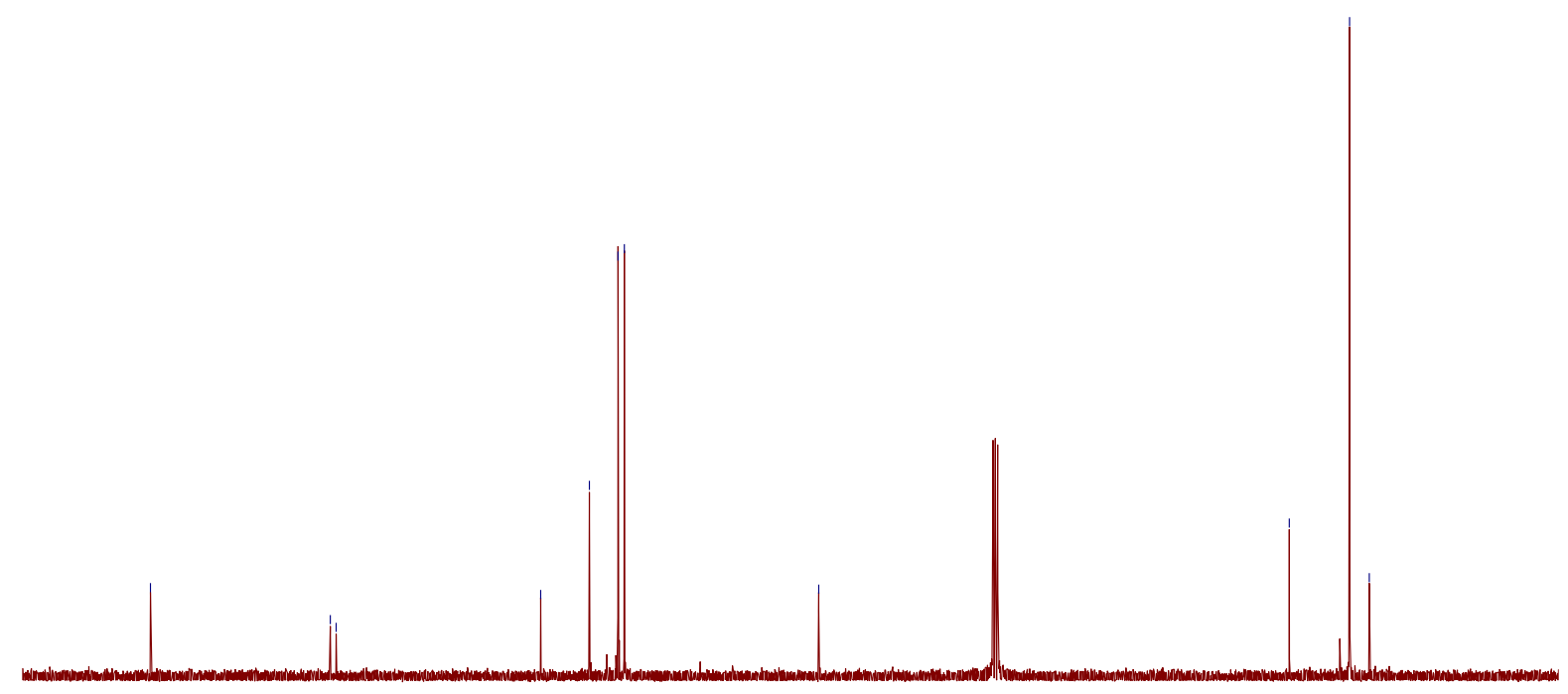

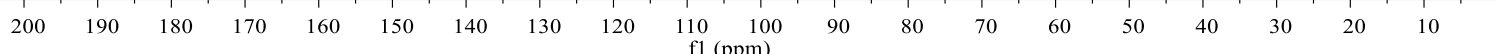

${ }^{1} \mathrm{H}$ and ${ }^{13} \mathrm{C}$ NMR spectra for product $4 p\left(\mathrm{CDCl}_{3}\right)$

S100 


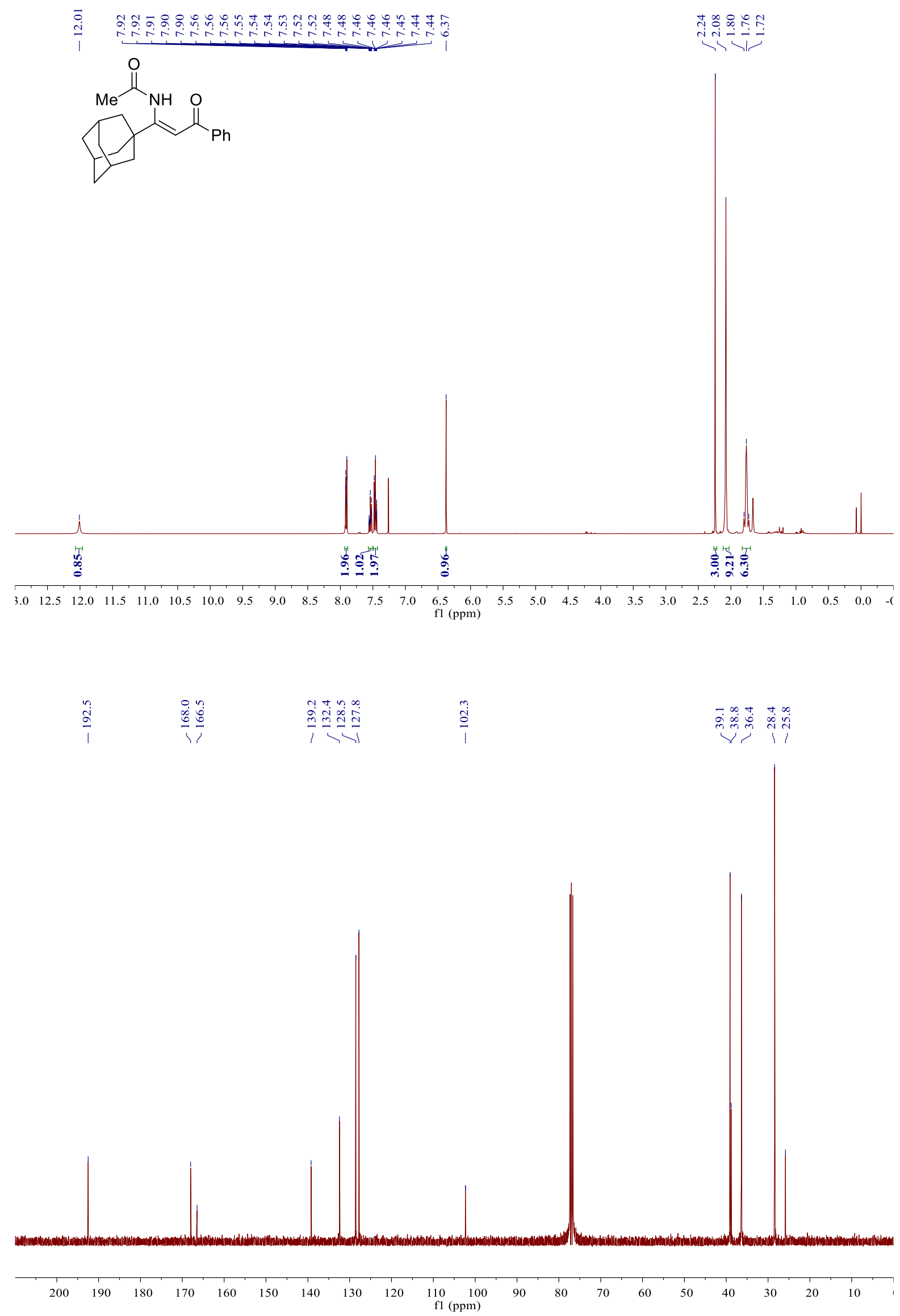

${ }^{1} \mathrm{H}$ and ${ }^{13} \mathrm{C}$ NMR spectra for product $4 \mathrm{q}\left(\mathrm{CDCl}_{3}\right)$

S101 


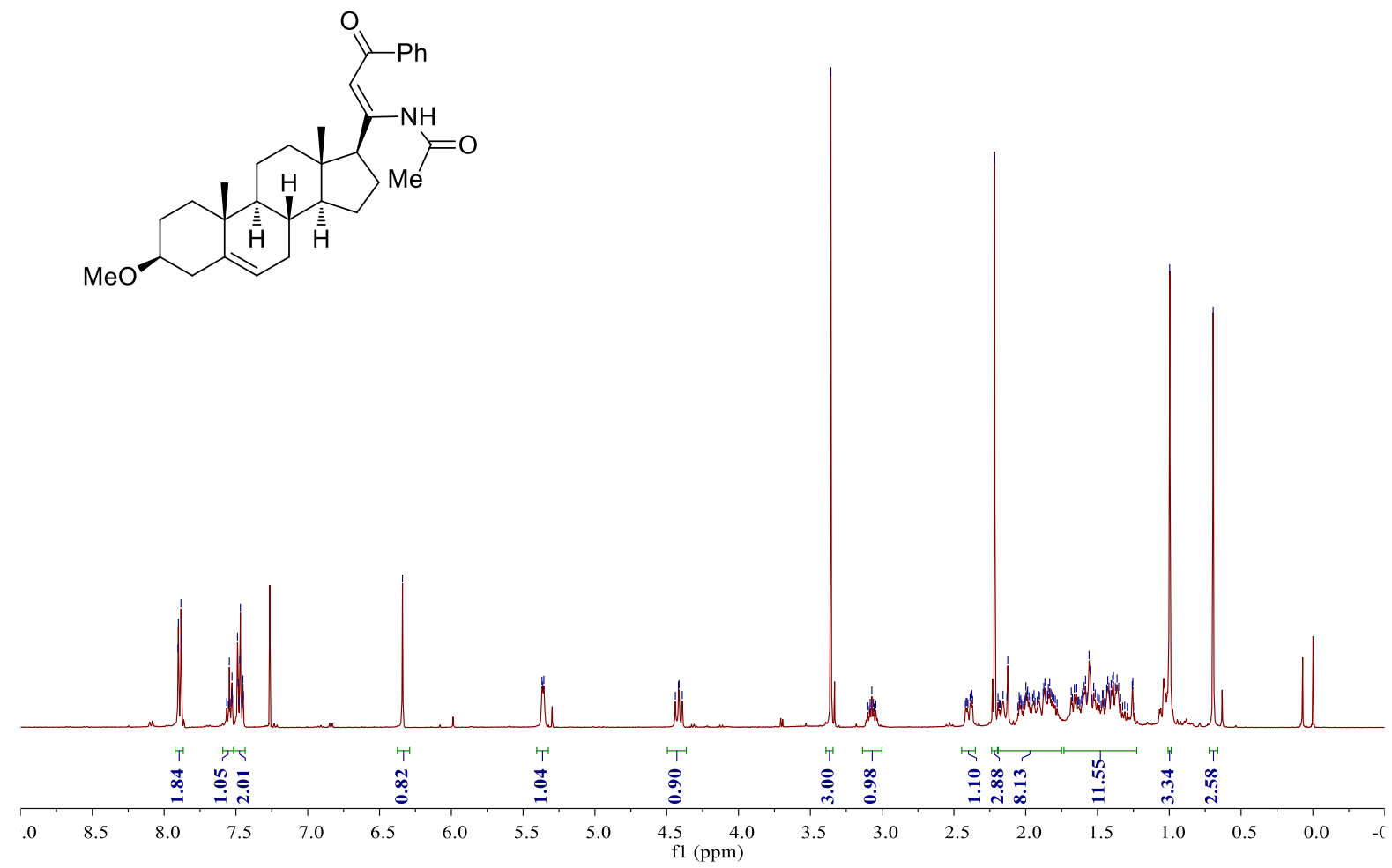

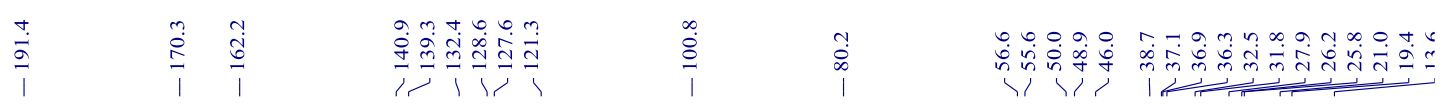

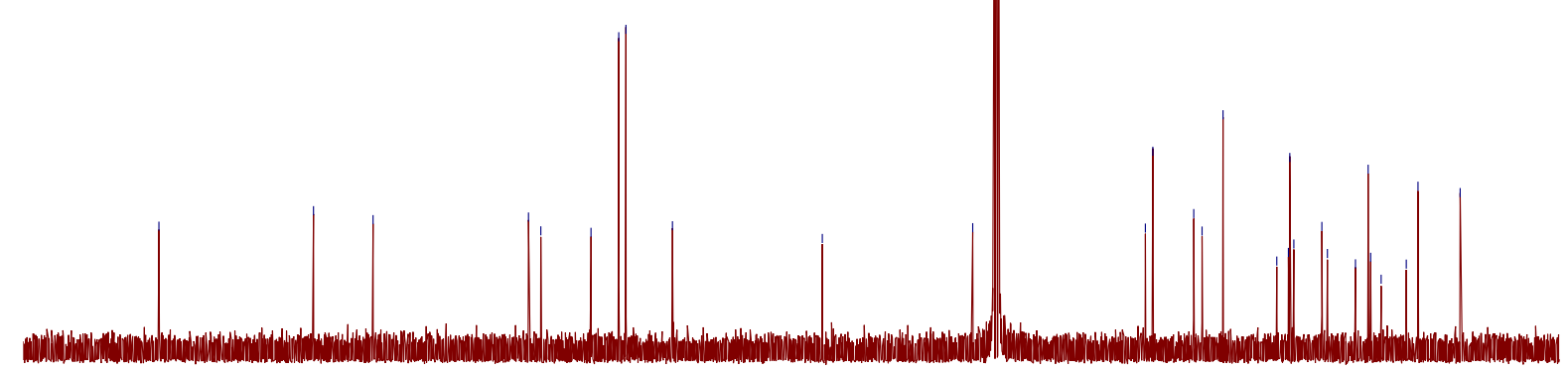

$\begin{array}{lllllllllll}200 & 190 & 180 & 170 & 160 & 150 & 140 & 130 & 120 & 110 \quad 100 \\ \text { f1 (ppm) }\end{array}$

${ }^{1} \mathrm{H}$ and ${ }^{13} \mathrm{C}$ NMR spectra for product $4 \mathrm{r}\left(\mathrm{CDCl}_{3}\right)$ 


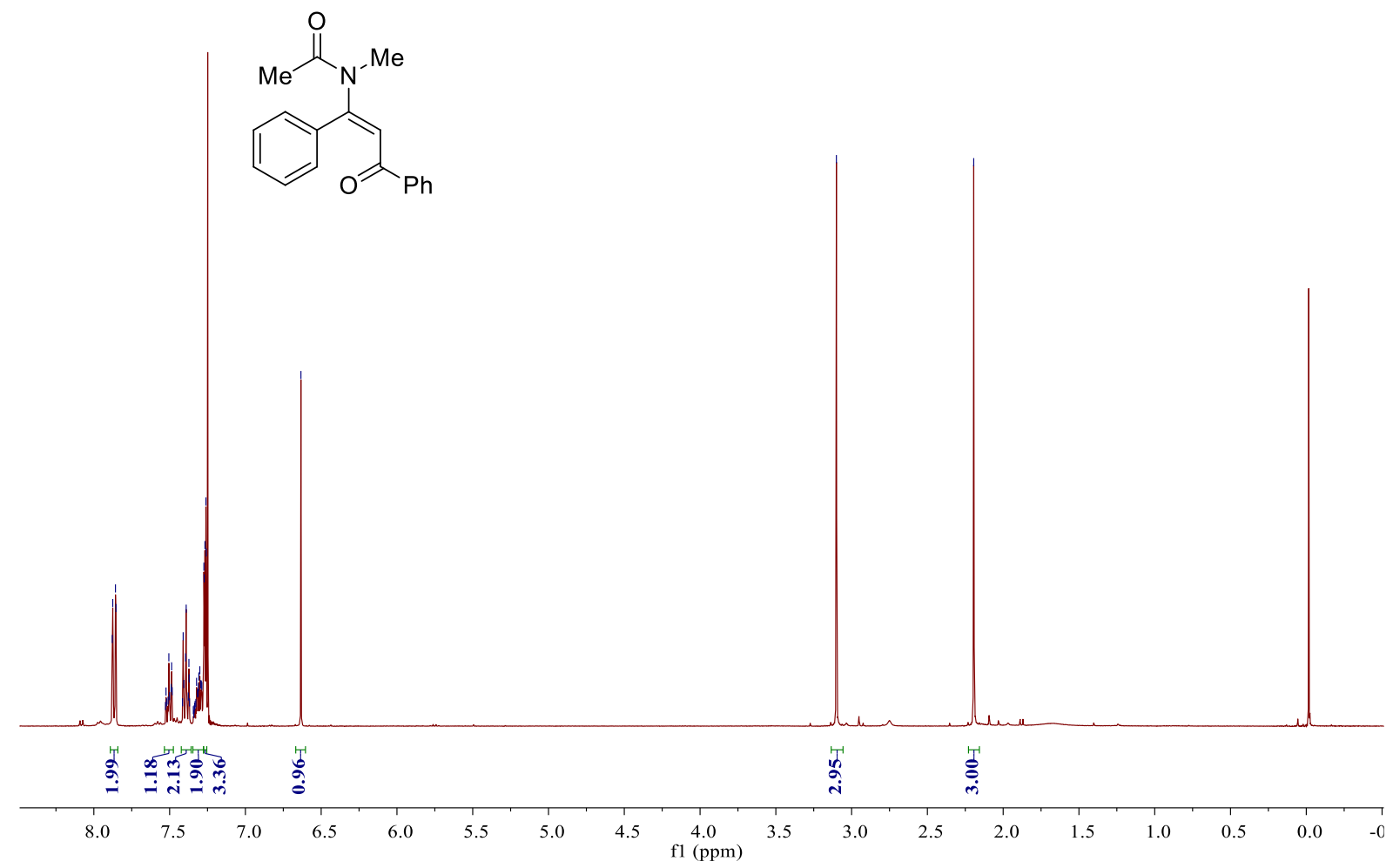

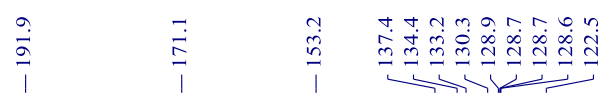

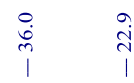

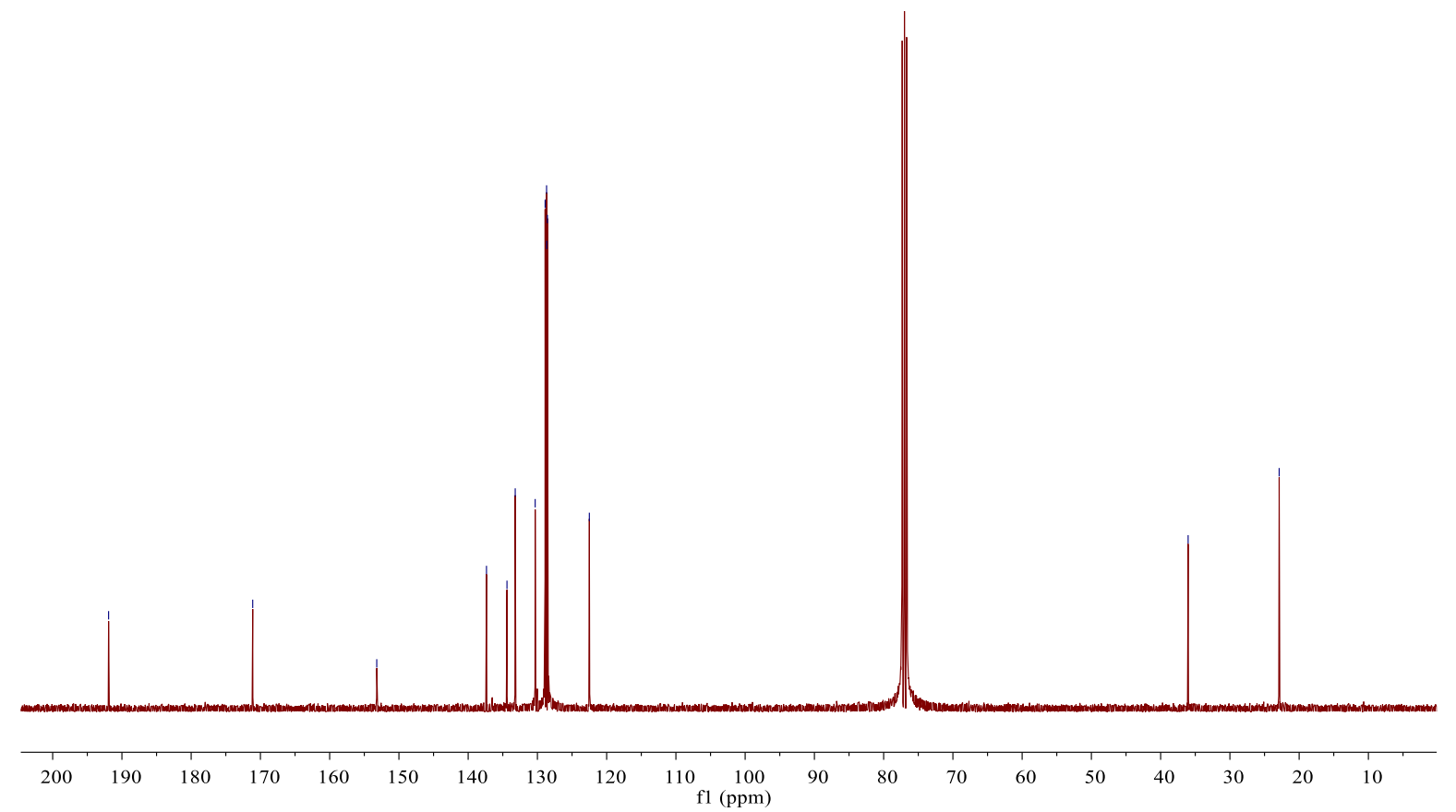

${ }^{1} \mathrm{H}$ and ${ }^{13} \mathrm{C}$ NMR spectra for product $4 \mathrm{~s}\left(\mathrm{CDCl}_{3}\right)$ 


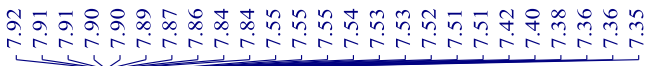



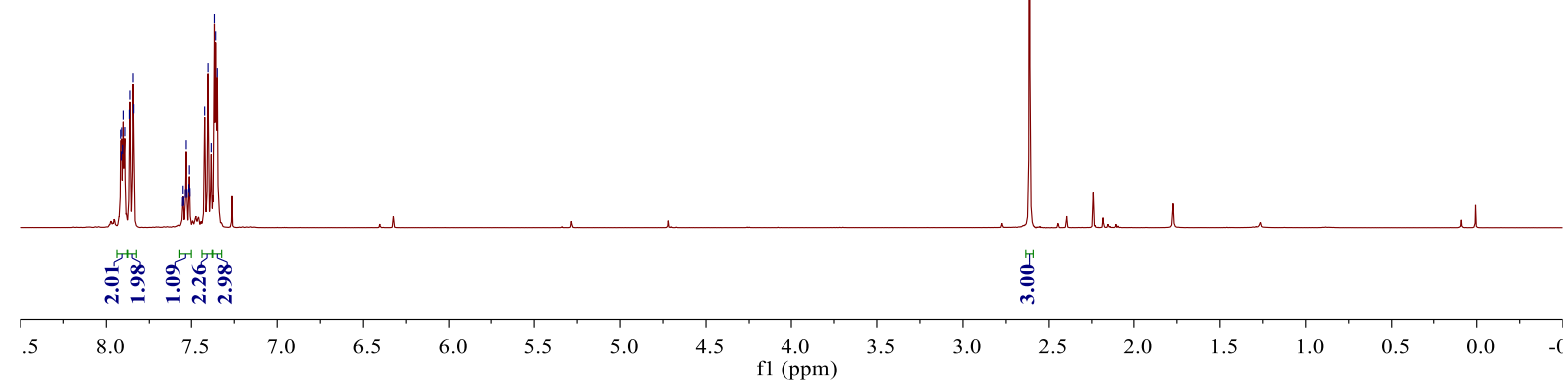

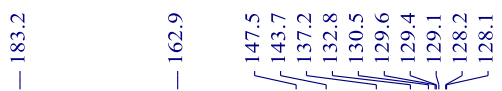

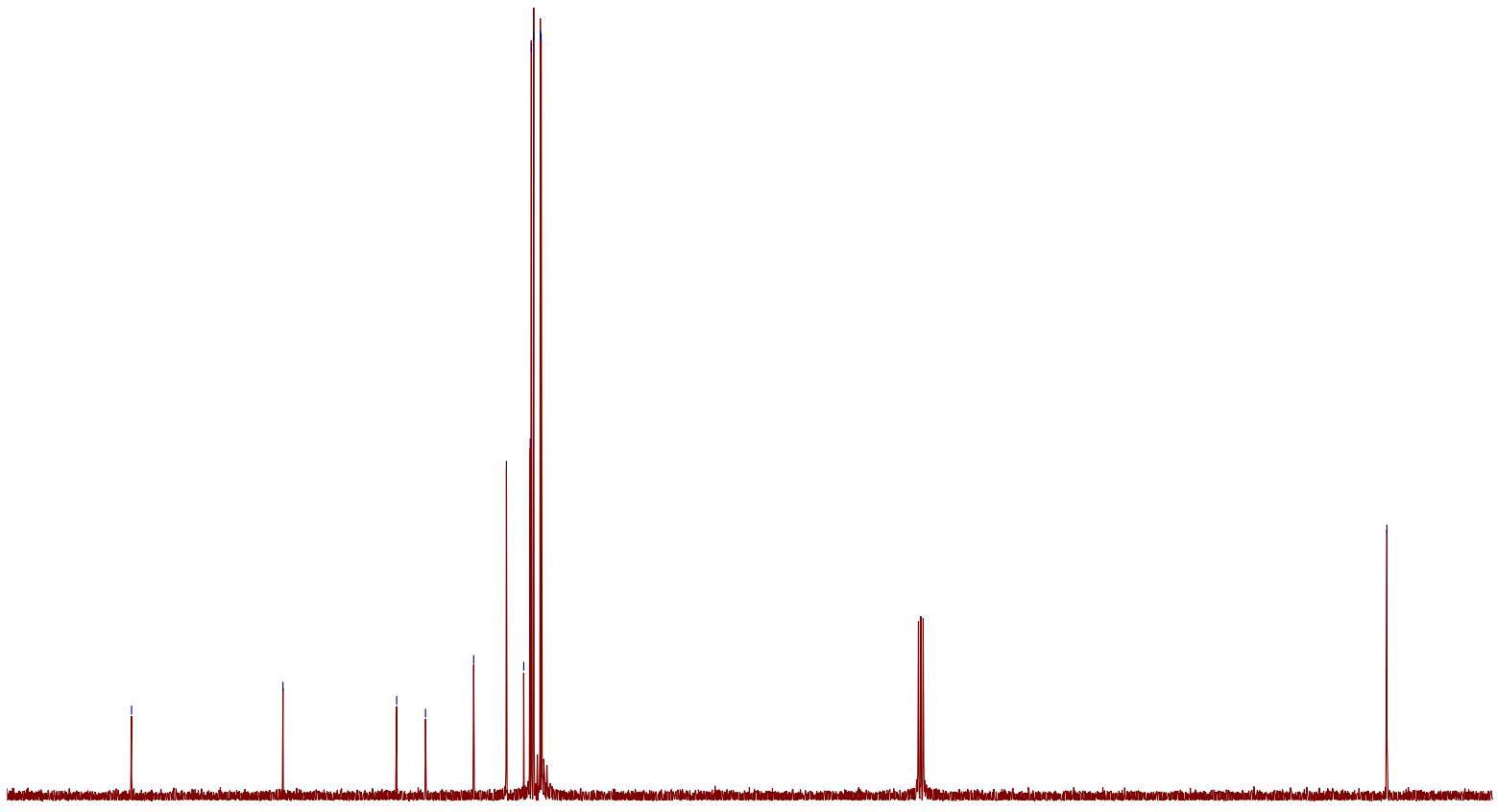

$190 \quad 180$

150

13

120

$10 \begin{array}{cc}100 \\ \mathrm{f} 1(\mathrm{ppm})\end{array}$

${ }^{1} \mathrm{H}$ and ${ }^{13} \mathrm{C}$ NMR spectra for product $5 \mathrm{a}\left(\mathrm{CDCl}_{3}\right)$ 


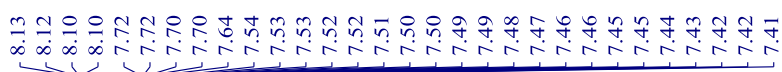<smiles>Cc1c(-c2ccccc2)cc(-c2ccccc2)nc1-c1ccccc1</smiles>

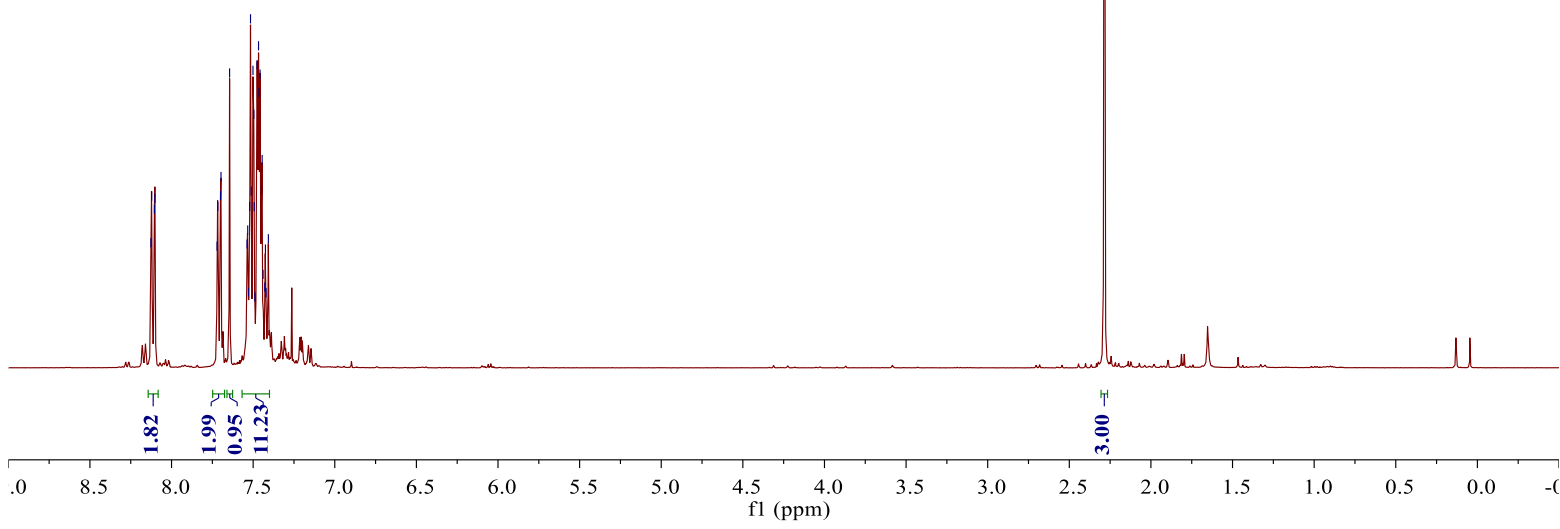

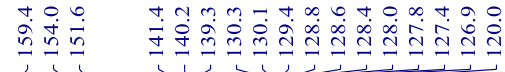

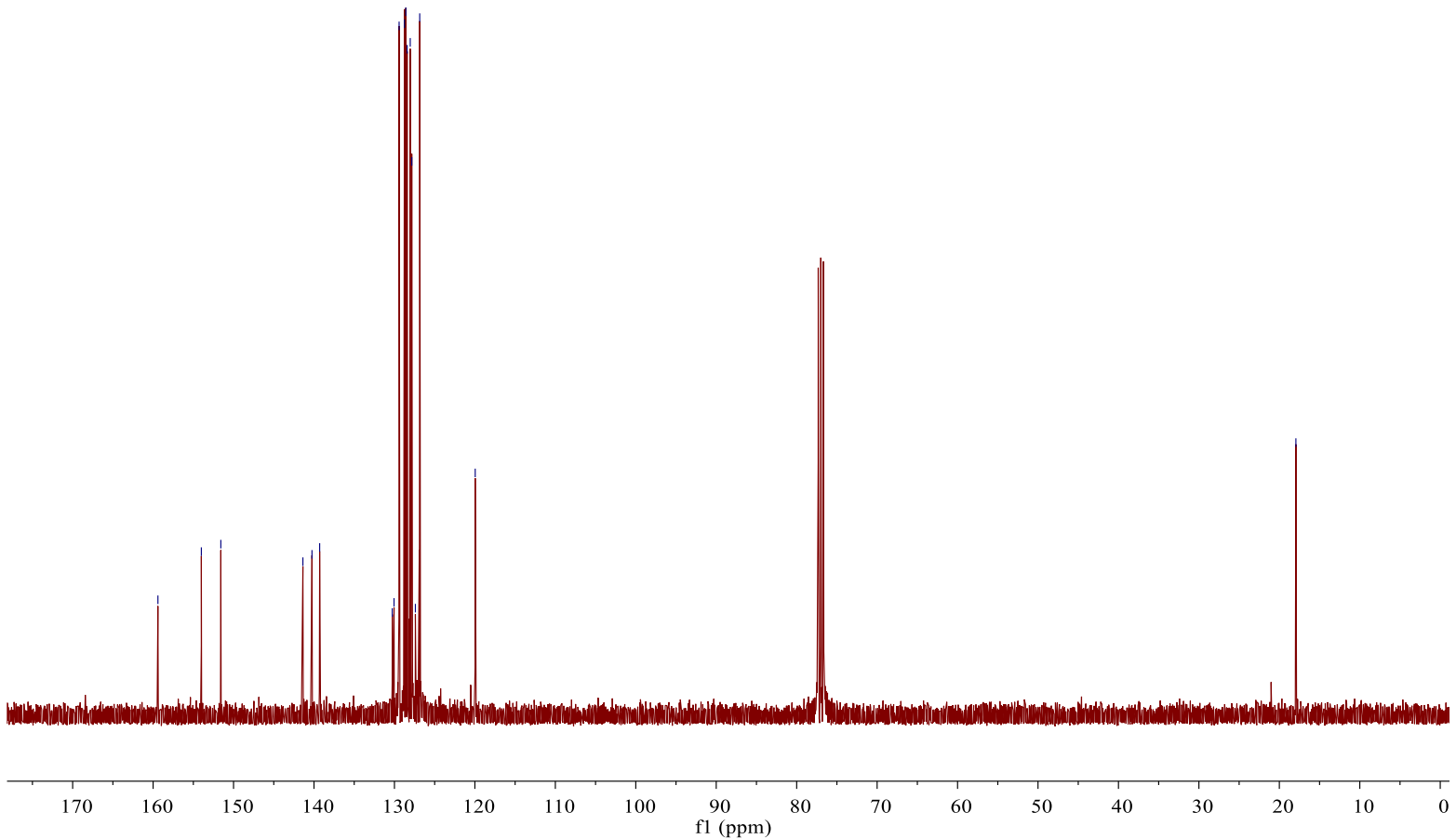

${ }^{1} \mathrm{H}$ and ${ }^{13} \mathrm{C}$ NMR spectra for product $5 \mathrm{~b}\left(\mathrm{CDCl}_{3}\right)$ 
<smiles>O=c1cc(-c2ccccc2)cc(-c2ccccc2)[nH]1</smiles>

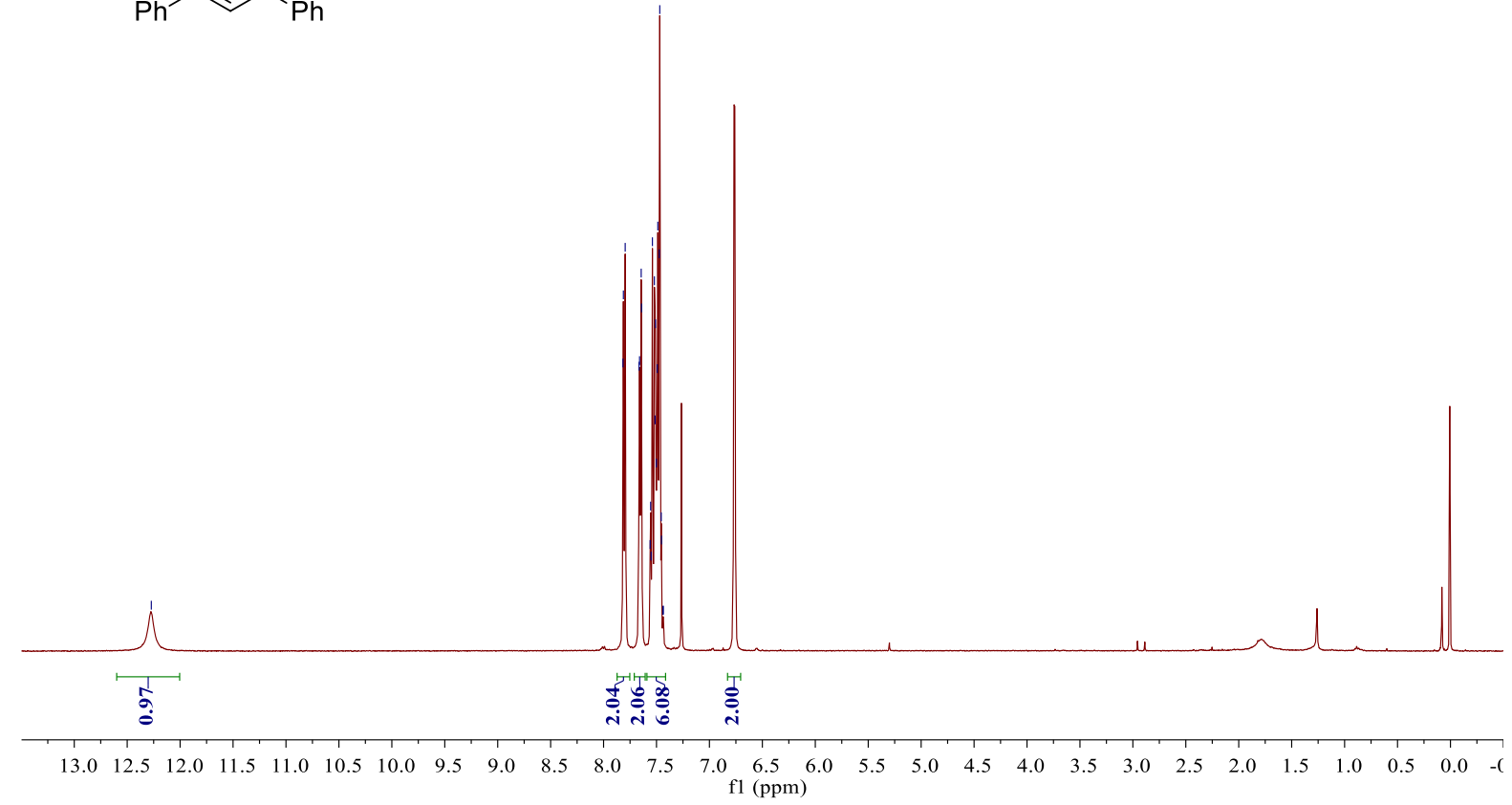

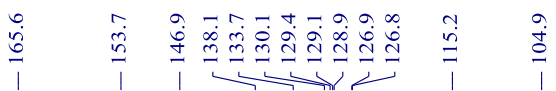

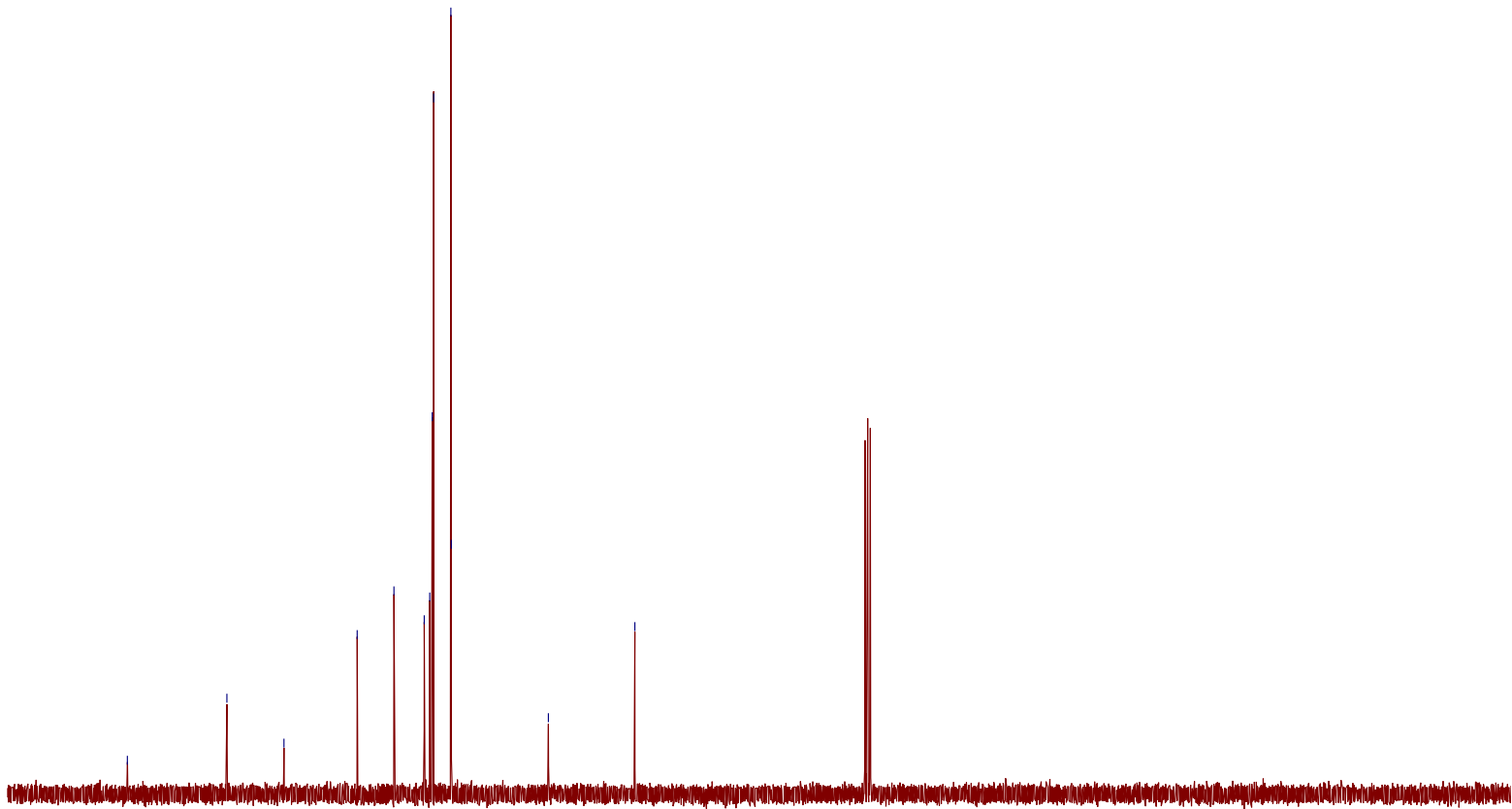

$\begin{array}{llll}170 & 160 & 150 & 140\end{array}$

$130 \quad 120$

100

90
f1 $(\mathrm{ppm})$

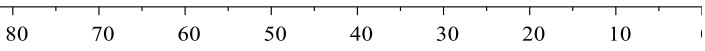

${ }^{1} \mathrm{H}$ and ${ }^{13} \mathrm{C}$ NMR spectra for product $5 \mathrm{c}\left(\mathrm{CDCl}_{3}\right)$ 
$\underbrace{\underbrace{2}}$
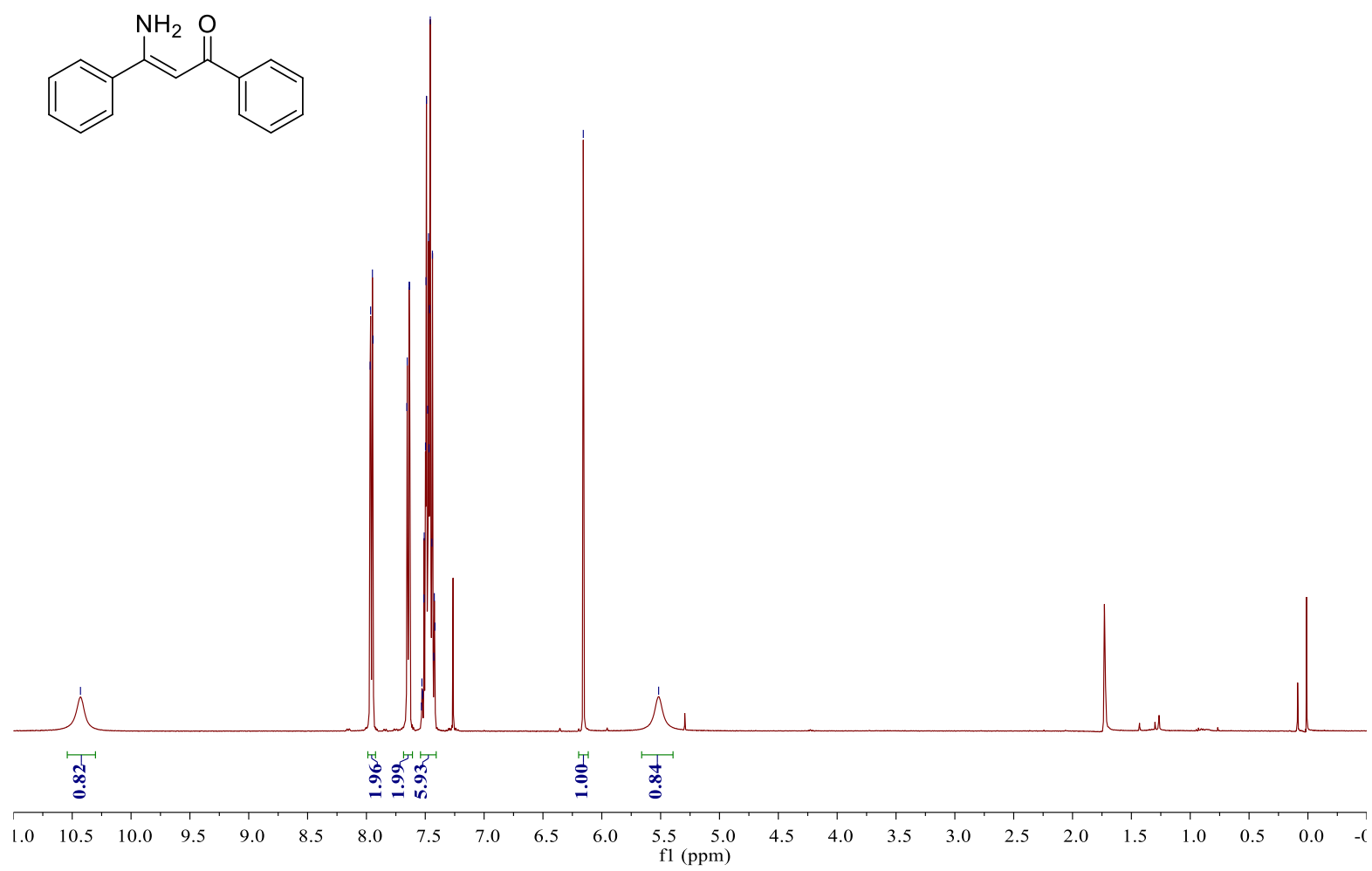

$\stackrel{1}{\frac{1}{2}}$

$\stackrel{\text { ठें }}{\text { । }}$

$\underbrace{-\underbrace{2}}$

$\stackrel{े}{i}$

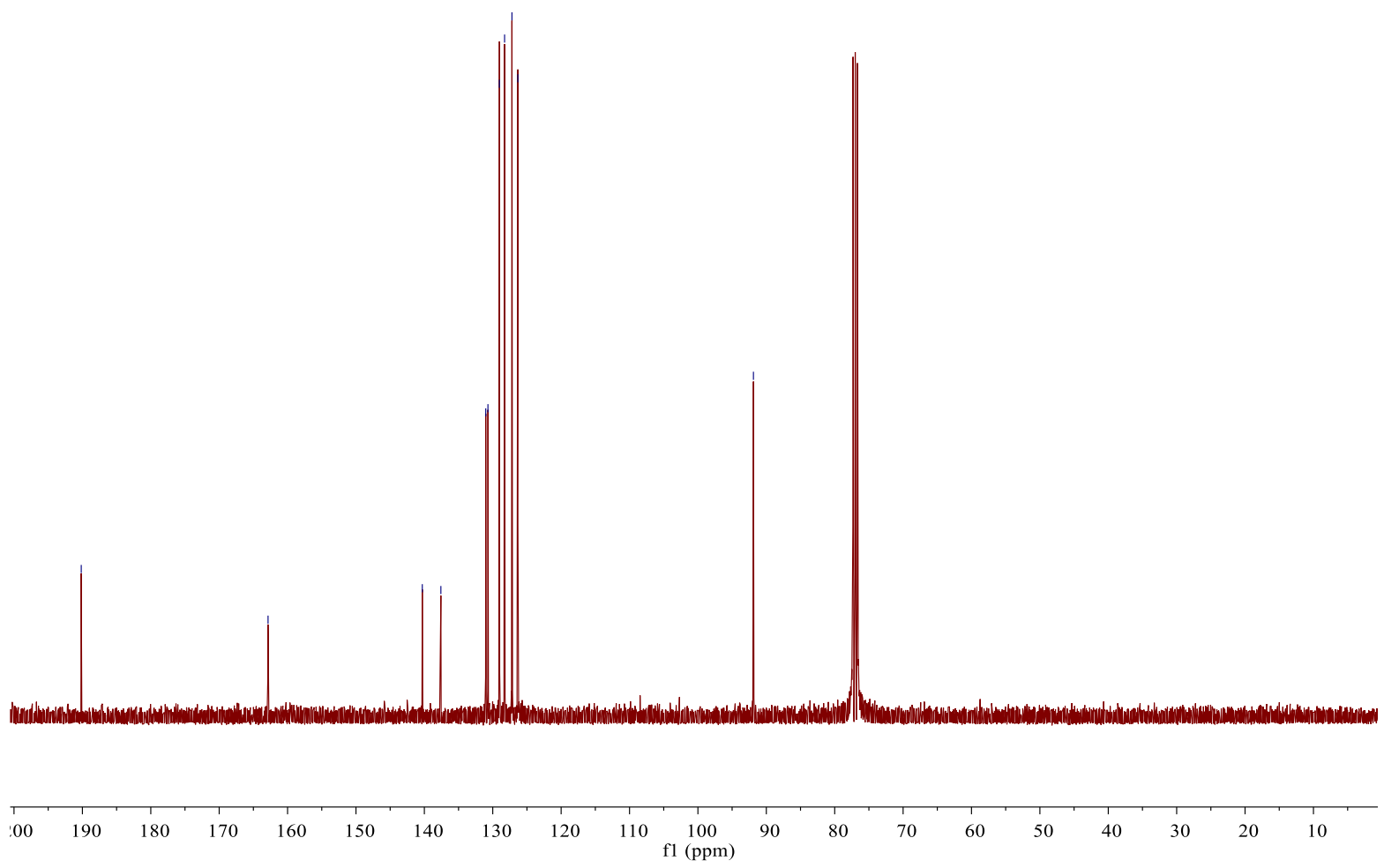

${ }^{1} \mathrm{H}$ and ${ }^{13} \mathrm{C}$ NMR spectra for product $5 \mathrm{~d}\left(\mathrm{CDCl}_{3}\right)$

S107 


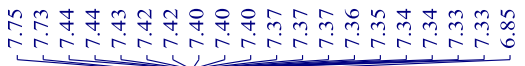

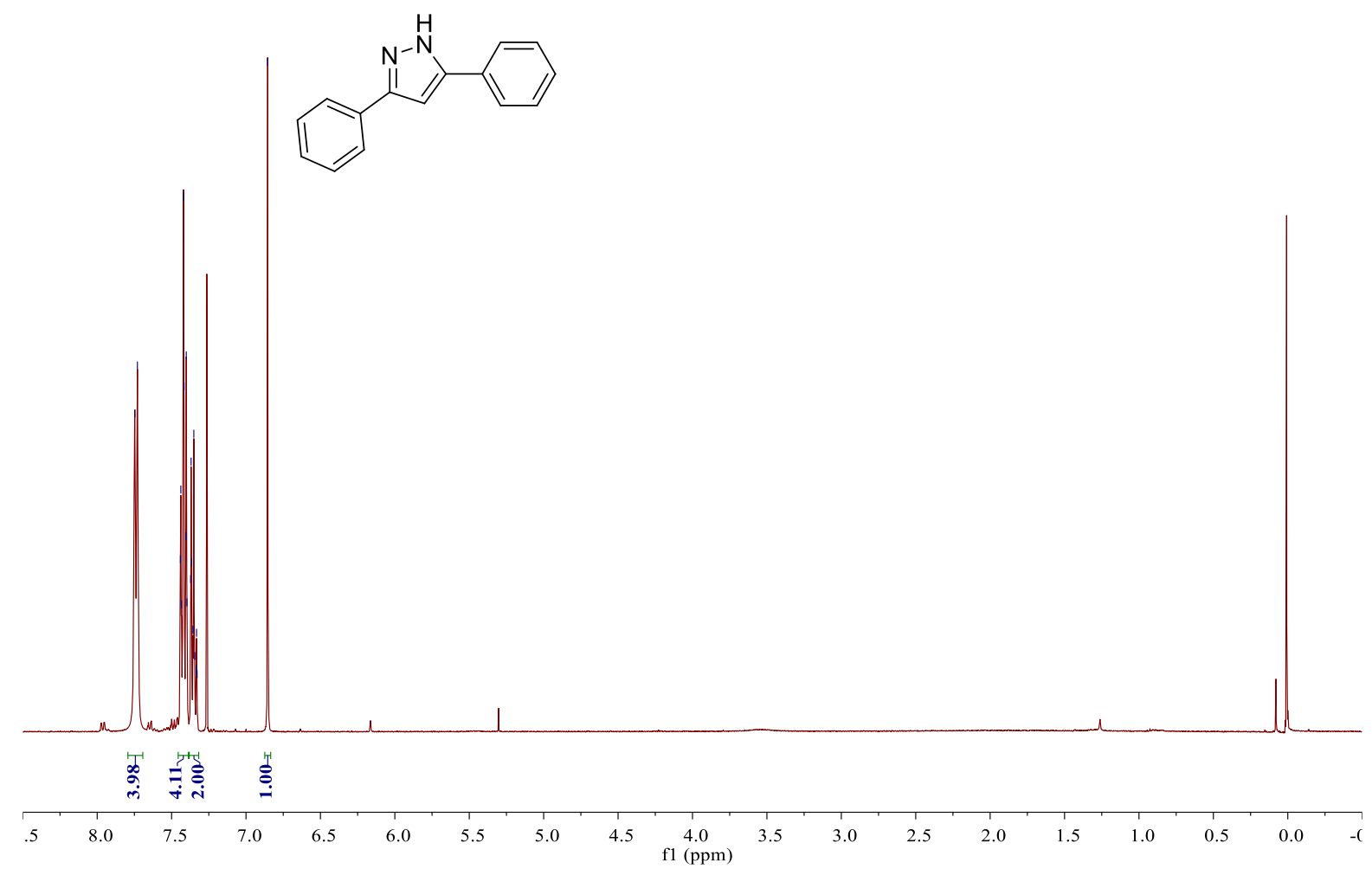

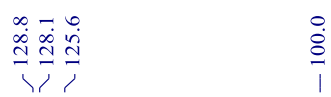

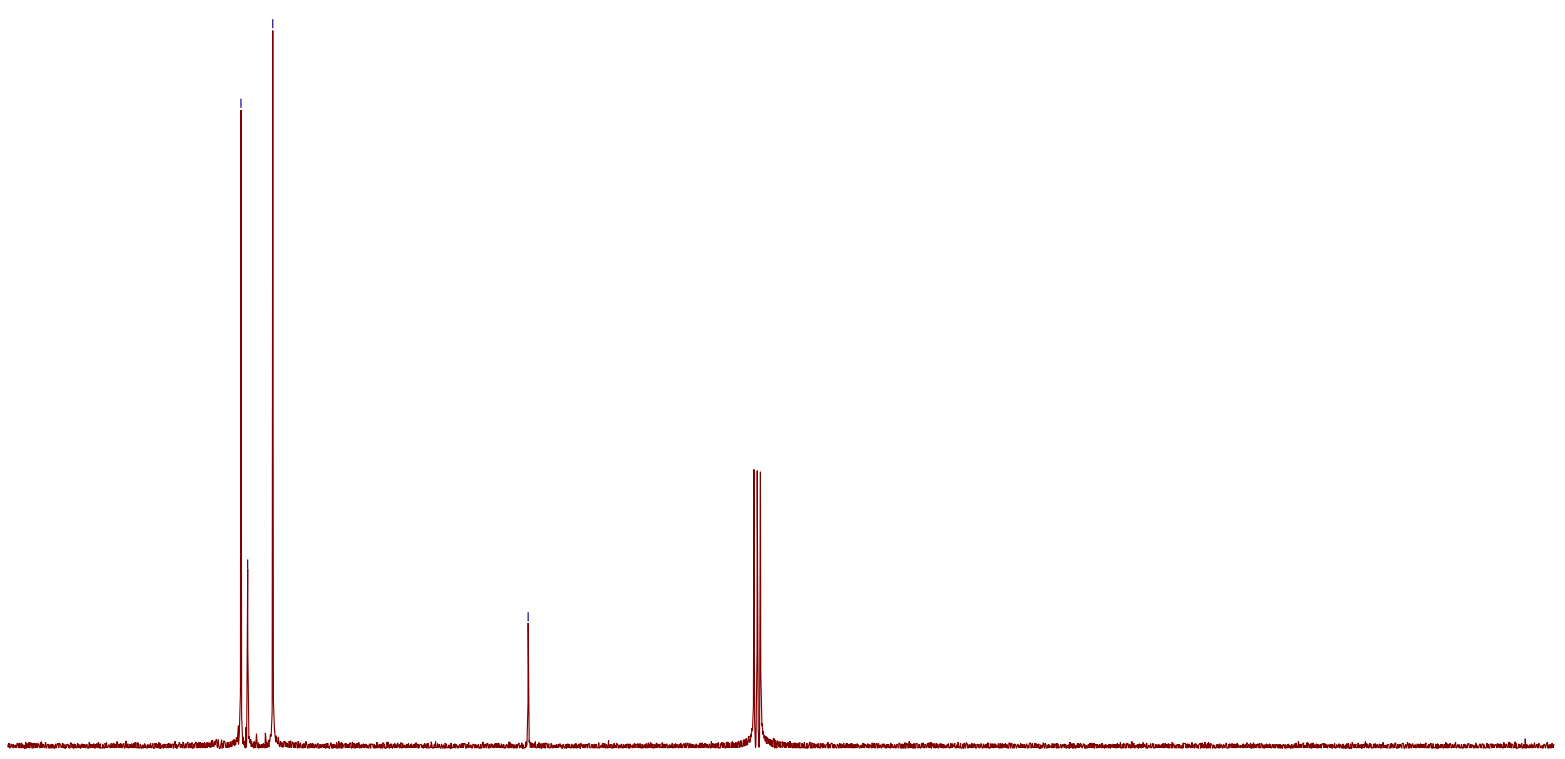

$\begin{array}{llllllllllllllllllllllllllllllllllllllllll}150 & 145 & 140 & 135 & 130 & 125 & 120 & 115 & 110 & 105 & 100 & 95 & 90 & 85 & 80 & 75 & 70 & 65 & 60 & 55 & 50 & 45 & 40 & 35 & 30 & 25 & 20 & 15 & 10 & 5 & 0\end{array}$

${ }^{1} \mathrm{H}$ and ${ }^{13} \mathrm{C}$ NMR spectra for product $5 \mathrm{e}\left(\mathrm{CDCl}_{3}\right)$ 
<smiles>CC(=O)NC(CC(O)c1ccccc1)c1ccccc1</smiles>
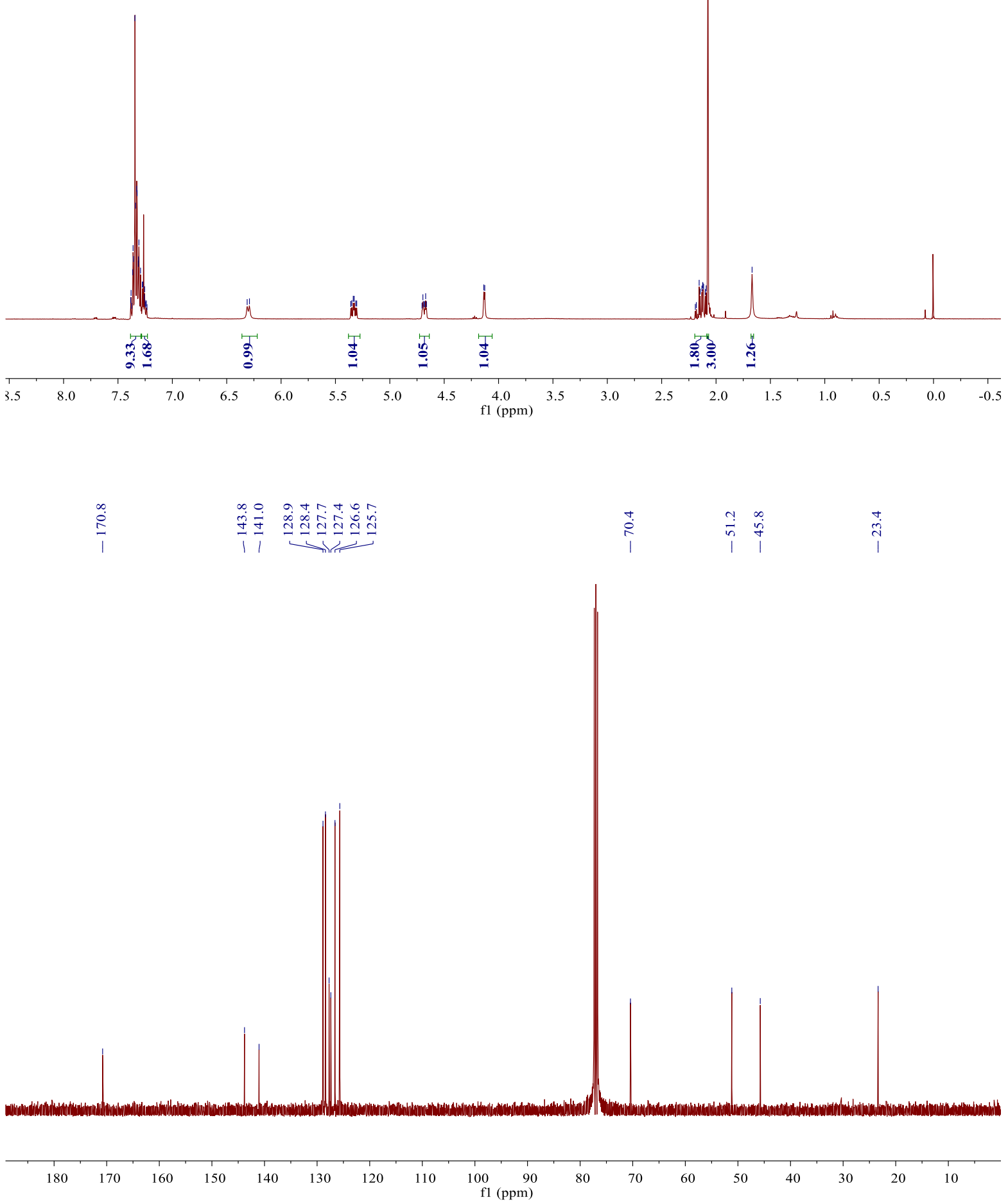